Stiftung Haus der kleinen Forscher (Hrsg.)

Diemut Kucharz, Lisa Öz, Julia Katharina Schmidt, Nina Skorsetz, Mirjam Steffensky

\title{
Professionalisierung pädagogischer Fach- und Lehrkräfte in der frühen MINT-Bildung
}

Wissenschaftliche Untersuchungen zur Arbeit der Stiftung „Haus der kleinen Forscher“

Verlag Barbara Budrich B:

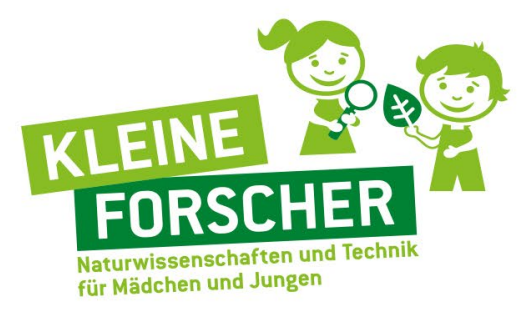


Professionalisierung pädagogischer Fach- und Lehrkräfte in der frühen MINT-Bildung

Stiftung Haus der kleinen Forscher:

GEFÖRDERT VOM PARTNER

Bundesministerium Helmholtz-Gemeinschaft

für Bildung Siemens Stiftung

und Forschung Dietmar Hopp Stiftung

Deutsche Telekom Stiftung 
Wissenschaftliche Untersuchungen zur Arbeit der Stiftung „Haus der kleinen Forscher“

Band 13 
Stiftung Haus der kleinen Forscher (Hrsg.)

\section{Professionalisierung pädagogischer Fach- und Lehrkräfte in der frühen MINT-Bildung}

Diemut Kucharz, Lisa Öz, Julia Katharina Schmidt, Nina Skorsetz

Mit einem Geleitwort von Mirjam Steffensky

Verlag Barbara Budrich

Opladen • Berlin • Toronto 2020 
Herausgeber: Stiftung Haus der kleinen Forscher

Verantwortlich: Dr. Janna Pahnke

Projektleitung: Dr. Elena Harwardt-Heinecke

Konzeption und Redaktion: Lisa Gerloff, Dr. Claudia Schiefer

Redaktionelle Mitarbeit: Karin Griffiths, Jörn Gründler, Julia Rienow

Weitere Informationen finden Sie unter: www.haus-der-kleinen-forscher.de

Haben Sie Fragen, Anmerkungen oder Anregungen zu diesem Band oder der wissenschaftlichen Begleitung der Stiftungsarbeit?

Wenden Sie sich an: forschung@haus-der-kleinen-forscher.de.

Weitere Informationen und Studienergebnisse finden Sie auch unter

www.haus-der-kleinen-forscher.de, Rubrik Wissenschaftliche Begleitung.

Bibliografische Information der Deutschen Nationalbibliothek

Die Deutsche Nationalbibliothek verzeichnet diese Publikation in der Deutschen Nationalbibliografie; detaillierte bibliografische Daten sind im Internet über http://dnb.d-nb.de abrufbar.

(C) 2020 Dieses Werk ist im Verlag Barbara Budrich erschienen und steht unter folgender Creative Commons Lizenz:

http://creativecommons.org/licenses/by-nc-nd/3.0/de/

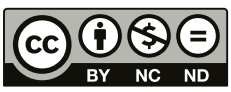

Verbreitung, Speicherung und Vervielfältigung erlaubt, kommerzielle Nutzung und Veränderung nur mit Genehmigung des Verlags Barbara Budrich

Ausgenommen von dieser Lizenz sind alle verwendeten Abbildungen, deren Verwertung außerhalb der engen Grenzen des Urheberrechtsgesetzes ohne Zustimmung des Verlages unzulässig und strafbar ist. Das gilt insbesondere für Vervielfältigungen, Übersetzungen, Mikroverfilmungen und die Einspeicherung und Verarbeitung in elektronischen Systemen.

Dieses Buch steht im Open-Access-Bereich der Verlagsseite zum kostenlosen Download bereit (http://dx.doi.org/10.3224/84742320).

Eine kostenpflichtige Druckversion kann über den Verlag bezogen werden. Die Seitenzahlen in der Druck- und Onlineversion sind identisch.

$$
\begin{array}{ll}
\text { ISBN } & 978-3-8474-2391-1 \text { (Paperback) } \\
\text { eISBN } & 978-3-8474-1578-7 \text { (eBook) } \\
\text { DOI } & 10.3224 / 84742391
\end{array}
$$

Umschlaggestaltung: Bettina Lehfeldt, Kleinmachnow - www.lehfeldtgraphic.de Titelbildnachweis: Birte Filmer/Stiftung Haus der kleinen Forscher Lektorat und Satz: Ulrike Weingärtner, Gründau; info@textakzente.de Druck: Medienhaus Plump, Rheinbreitbach

Printed in Europe, gedruckt auf FSC-zertifiziertem Papier 


\section{Inhaltsverzeichnis}

Informationen über die Autorinnen.................. 9

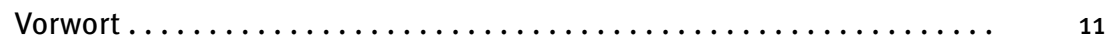

Geleitwort............................. 12

Mirjam Steffensky

Einleitung ...................................... 16

Stiftung Haus der kleinen Forscher

1 Überblick zur Stiftung „Haus der kleinen Forscher“ ........... 17

2 Die Bedeutung der Fort- und Weiterbildung in der frühen (MINT-)Bildung $\ldots \ldots \ldots \ldots \ldots \ldots \ldots \ldots \ldots \ldots \ldots \ldots \ldots \ldots \ldots \ldots \ldots$

3 Wirkungen der Bildungsangebote der Initiative „Haus der kleinen Forscher“ .................... 30

4 Hintergründe der externen Studie und stiftungsinternen

Befragung zur Professionalisierung von pädagogischen

Fach- und Lehrkräften. ..................... 33

Zusammenfassung zentraler Ergebnisse $\ldots \ldots \ldots \ldots \ldots \ldots \ldots \ldots .38$

Stiftung Haus der kleinen Forscher

A Entwicklungsverläufe von pädagogischen Fach- und Lehrkräften

in der frühen MINT-Bildung ..................... 46

Nina Skorsetz, Lisa Öz, Julia Katharina Schmidt, Diemut Kucharz

$1 \quad$ Einleitung $\ldots \ldots \ldots \ldots \ldots \ldots \ldots \ldots \ldots \ldots \ldots \ldots \ldots \ldots \ldots \ldots \ldots \ldots \ldots, 47$

2 Theoretischer Hintergrund. .................... 49

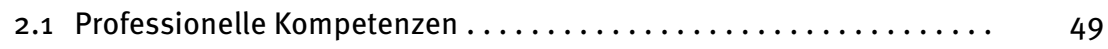

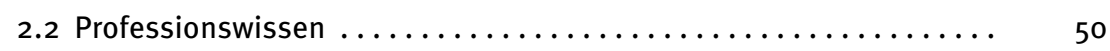

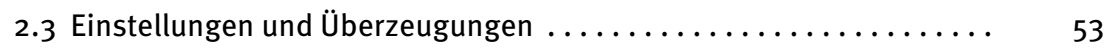

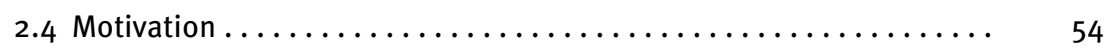

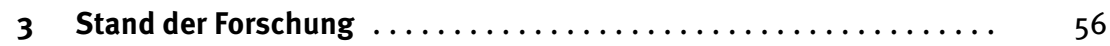

3.1 Entwicklungsverläufe durch Nutzung von Lerngelegenheiten ..... 56

3.2 MINT-spezifische Studien zu Professionswissen, Motivation und

Einstellungen............................ 58

3.3 Forschungsdesiderate und daraus folgende

Untersuchungsschwerpunkte.................... 59 
4 Forschungsfragen $\ldots \ldots \ldots \ldots \ldots \ldots \ldots \ldots \ldots \ldots \ldots \ldots \ldots \ldots \ldots \ldots \ldots \ldots \ldots$

5 Forschungsdesign und Stichprobenziehung. ............. 62

6 Instrumentenentwicklung und Durchführung.............. 64

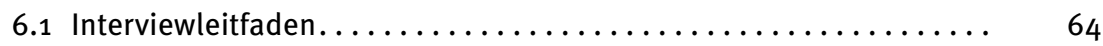

6.2 Ableitung von Items aus der Interviewbefragung ............ 66

6.3 Modellierung der Items zu Entwicklungsverläufen ............ 68

6.4 Pilotierung des Fragebogens $\ldots \ldots \ldots \ldots \ldots \ldots \ldots \ldots \ldots \ldots \ldots \ldots \ldots \ldots$

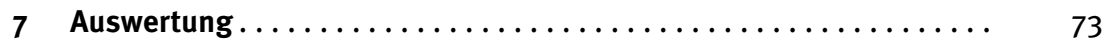

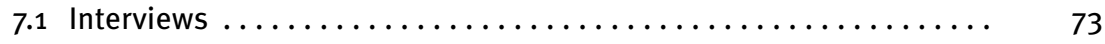

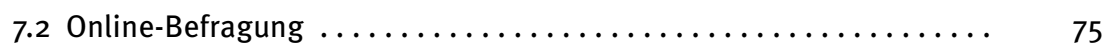

8 Ergebnisse der Studie ......................... 80

8.1 Stand der Professionalisierung im MINT-Bereich -

Beantwortung der Unter-Forschungsfragen. ........... 80

8.2 Entwicklungsverläufe im Professionalisierungsprozess -

Beantwortung der Hauptforschungsfragen . . . . . . . . . . . 107

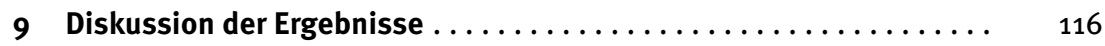

9.1 Professionswissen: Stand und Entwicklung. ............. 116

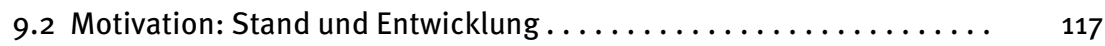

9.3 Einstellungen: Stand und Entwicklung. .............. 118

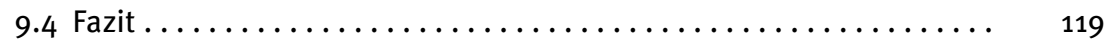

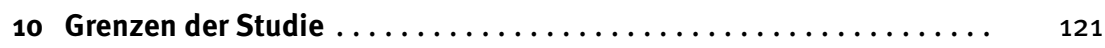

11 Schlussfolgerungen für die Arbeit der Stiftung

„Haus der kleinen Forscher“.................... 123

B Kompetenzentwicklung von Pädagoginnen und Pädagogen

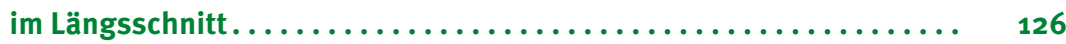

Stiftung Haus der kleinen Forscher

1 Hintergrund der Längsschnittanalysen zu Wirkungen des „Haus der kleinen Forscher“-Fortbildungsangebots . . . . . . . 127

1.1 Inhaltlich-pädagogische Ziele des Fortbildungsprogramms ...... 127

1.2 Wirkungsmodell der Kompetenzentwicklung . . . . . . . . . . . . 127

1.3 Bisherige Wirkungsanalysen zum Fortbildungsprogramm ......... 129

1.4 Erkenntnisinteresse der vorliegenden Analysen ............ 131

2 Design und Stichprobenbeschreibung der Längsschnittbefragung . . 132

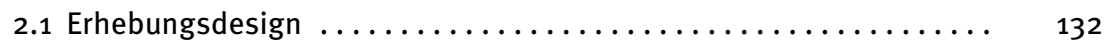

2.2 Übersicht über die verwendeten Konstrukte und Messinstrumente . . 133 
2.3 Stichprobenbeschreibung $\ldots \ldots \ldots \ldots \ldots \ldots \ldots \ldots \ldots \ldots \ldots . . \ldots \ldots \ldots . \ldots \ldots$

2.4 Analyseverfahren ............................ 144

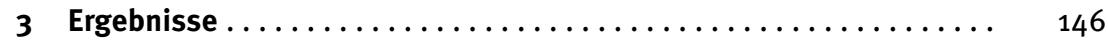

3.1 Kurz- und mittelfristige Kompetenzentwicklung nach einem

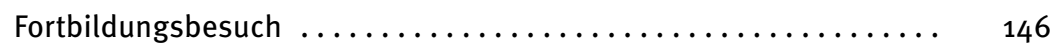

3.2 Zusammenhänge individueller Eigenschaften und

organisationaler Rahmenbedingungen mit den Wirkungen

der Fortbildungsteilnahme ..................... 149

3.3 Zusammenhänge der Fortbildungsanzahl und -frequenz

mit dem Ausgangsniveau verschiedener Kompetenzen . . . . . . . 158

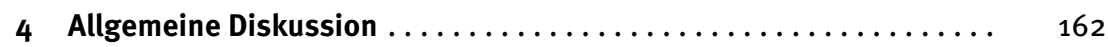

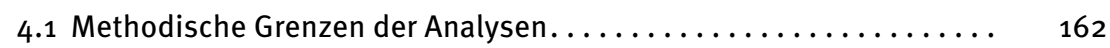

4.2 Gesamtzusammenfassung und Ausblick .............. 163

Fazit und Ausblick - Wie die Stiftung „Haus der kleinen Forscher“

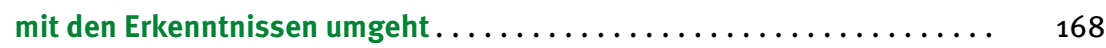

Stiftung Haus der kleinen Forscher

1 Ergebnisse der Untersuchungen und ihre Bedeutung

für die Stiftungsarbeit. . . . . . . . . . . . . . . . . . 169

2 Grenzen der Untersuchungsergebnisse $\ldots \ldots \ldots \ldots \ldots \ldots \ldots . \ldots \ldots$

3 Umgang mit den Empfehlungen und Erkenntnissen aus den

Untersuchungen ........................... 174

4 Ausblick - weitere wissenschaftliche Begleitung und

Erkenntnisbedarfe......................... 193

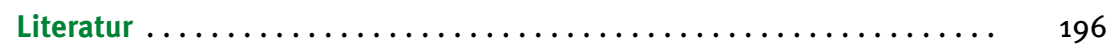

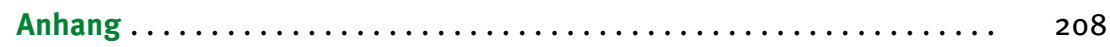

Bildquellenverzeichnis ....................... 229

Über die Stiftung „Haus der kleinen Forscher“ $\ldots \ldots \ldots \ldots \ldots \ldots \ldots .230$

Bisher erschienen in der Wissenschaftlichen Schriftenreihe

der Stiftung „Haus der kleinen Forscher" $\ldots \ldots \ldots \ldots \ldots \ldots \ldots \ldots .232$ 



\section{Informationen über die Autorinnen}

\section{Prof. Dr. Diemut Kucharz}

Goethe-Universität Frankfurt am Main, Fachbereich Erziehungswissenschaften, Institut für Pädagogik der Elementar- und Primarstufe

Arbeitsschwerpunkte: Sachunterricht, Elementarbildung, Offener Unterricht, Jahrgangsübergreifendes Lernen, Sprachförderung, Unterrichtsforschung, Schulentwicklung, Inklusion

Kontakt: Theodor-W.-Adorno-Platz 6, 60629 Frankfurt am Main, kucharz@em.uni-frankfurt.de

\section{Dr. Lisa Öz}

Stadt Dietzenbach, Abteilung Jugendhilfe/Soziale Arbeit (vormals: Goethe-Universität Frankfurt am Main, Fachbereich Erziehungswissenschaften, Institut für Pädagogik der Elementar- und Primarstufe)

Arbeitsschwerpunkte: Naturwissenschaftliche Elementarbildung, Teamarbeit von Lehrenden, Jugendvielfalt, Jugendbeteiligung

Kontakt: Justus-von-Liebig-Str. 19, 63128 Dietzenbach, lisa.oez@dietzenbach.de

\section{Julia Katharina Schmidt}

Goethe-Universität Frankfurt am Main, Fachbereich Erziehungswissenschaften, Institut für Pädagogik der Elementar- und Primarstufe

Arbeitsschwerpunkte: Klassenklima, Inklusion, Unterrichtsforschung

Kontakt: Theodor-W.-Adorno-Platz 6, 60629 Frankfurt am Main,

Jul.Schmidt@em.uni-frankfurt.de

\section{Dr. Nina Skorsetz}

Goethe-Universität Frankfurt am Main, Fachbereich Erziehungswissenschaften, Institut für Pädagogik der Elementar- und Primarstufe Arbeitsschwerpunkte: Sachunterricht, Naturwissenschaftliche Bildung im Elementar- und Primarbereich, Inklusion, Unterrichtsforschung Kontakt: Theodor-W.-Adorno-Platz 6, 60629 Frankfurt am Main, skorsetz@em.uni-frankfurt.de 


\section{Prof. Dr. Mirjam Steffensky}

Universität Hamburg, Fakultät für Erziehungswissenschaft, Didaktik der gesellschaftswissenschaftlichen und mathematisch-naturwissenschaftlichen Fächer Arbeitsschwerpunkte: Naturwissenschaftliches Lehren und Lernen im Elementar- und Primarbereich, Fachbezogenes Professionswissen von Fachkräften und Grundschullehrpersonen, Entwicklung und Evaluation von Materialien für den Elementar- und Primarbereich

Kontakt: Von-Melle-Park 8, 20146 Hamburg, mirjam.steffensky@uni-hamburg.de 


\section{Vorwort}

\section{Liebe Leserinnen und Leser,}

eine Erzieherin hat unlängst zu mir gesagt: „Vielen Dank für die Inspiration, die ich regelmäßig vom ,Haus der kleinen Forscher' für meine Arbeit in der Kita bekomme!" So etwas spornt mich und meine Kolleginnen

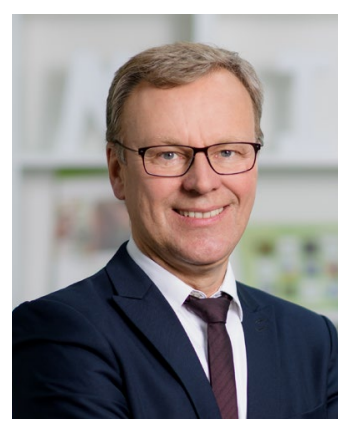
und Kollegen an, weiter an Fortbildungsangeboten für Erzieherinnen und Erzieher sowie für Lehr- und Leitungskräfte zu arbeiten, damit sie ihrerseits Mädchen und Jungen in Kitas, Horten und Grundschulen die alltägliche Begegnung mit Mathematik, Informatik, Naturwissenschaften und Technik - kurz MINT - sowie mit Fragen der Nachhaltigkeit ermöglichen können.

Wir möchten noch besser verstehen, was Pädagoginnen und Pädagogen brauchen, um sich in der frühen MINT-Bildung zu professionalisieren. Aus diesem Grund hat die Stiftung „Haus der kleinen Forscher“ gemeinsam mit dem Bundesministerium für Bildung und Forschung eine externe Studie gefördert und auch eine eigene Längsschnittbefragung gestartet. Eine Forschungsgruppe der GoetheUniversität Frankfurt am Main hat Einblicke in die professionelle Entwicklung pädagogischer Fach- und Lehrkräfte für den Bildungsbereich MINT gewonnen.

In ihrem Abschlussbericht kommt die Forschungsgruppe zu dem Ergebnis, dass der Besuch von Fortbildungen, gerade auch der wiederholte Besuch von Fortbildungen des „Hauses der kleinen Forscher“, zentral dafür ist, professionelle Kompetenzen in der frühen MINT-Bildung zu entwickeln. Fortgebildete Pädagoginnen und Pädagogen schätzen ihre Motivation, ihr Wissen und ihre Einstellung zu früher MINT-Bildung positiv ein. Eigene längsschnittliche Analysen bestätigen die Studienergebnisse und zeigen, wie bedeutsam auch die Rahmenbedingungen in der Organisation für die professionelle Entwicklung sind.

Diese Ergebnisse zeigen das positive Wirkungspotenzial unserer Arbeit. Gleichzeitig hat die Forschungsgruppe uns mitgegeben, dranzubleiben und unser Fortbildungsangebot fokussiert weiterzuentwickeln. Ein Auftrag, den wir gerne annehmen!

Ihr

Michael Fritz

Vorstandsvorsitzender der Stiftung „Haus der kleinen Forscher“ 


\section{Geleitwort}

von Mirjam Steffensky

Erneut hat sich die Stiftung „Haus der kleinen Forscher“ der Frage nach der Wirkung ihrer vielfältigen Aktivitäten gestellt. Wirkung ist hier im weiten Sinne von Entwicklungen und Veränderungen in Institutionen wie Kita und Grundschule, bei pädagogischen Fachkräften und - sicherlich am schwierigsten zu untersuchen bei Kindern aus Institutionen, die am Programm der Initiative „Haus der kleinen Forscher" teilnehmen, zu verstehen. Die kontinuierliche wissenschaftliche Untersuchung der Wirkung ihrer Aktivitäten ist für eine Initiative dieser Größe und vor allem Bedeutung angemessen, aber trotzdem keineswegs selbstverständlich. Im Rahmen dieses Bandes werden zwei wissenschaftliche Studien vorgestellt, in denen die Wirkung der Aktivitäten von einer externen sowie einer stiftungsinternen Forschungsgruppe untersucht werden. Die Stiftung schließt somit an die vorhergegangenen externen Studien an, sie baut aber auch ihr eigenes Monitoring aus. So wird der (vielleicht) stärker objektive Blick von außen und der Blick von innen durch Expertinnen und Experten, die die Prozesse im Detail kennen, klug verzahnt. Dadurch wird die Anschlussfähigkeit an die „Scientific Community“ der Bildungsforschung unterstützt und gleichzeitig die hohe Forschungsexpertise in der Stiftung und die Möglichkeit genutzt, im Prozess kontinuierlich Daten ökonomisch zu erheben und auszuwerten.

Thematisch wird in den hier vorgestellten Studien die professionelle Kompetenz von den Fortbildungsteilnehmerinnen und -teilnehmern, also den pädagogischen Fachkräften und Lehrkräften, in den Mittelpunkt gestellt. Diese stellen die zentrale Zielgruppe der Stiftung dar. Die angenommene Wirkungskette, die der Stiftungsarbeit zugrunde liegt, beschreibt die Wirkungen von Fortbildungen, die von geschulten Multiplikatorinnen und Multiplikatoren durchgeführt werden, auf die professionelle Kompetenz von Fach- und Lehrkräften, deren Handeln und damit auf die Entwicklung der „dazugehörigen“ Kita- und Schulkinder. Anders gesagt, bilden die pädagogischen Fach- und Lehrkräfte das „Bindeglied“ zwischen den Fortbildungen und der Bildungsqualität, die zur kindlichen Entwicklung beiträgt. Daher macht es Sinn, diese Gruppe und ihre Veränderungen im Zusammenhang mit der Teilnahme an Fortbildungen genauer zu untersuchen.

Anders als in bisherigen Begleitstudien wird nun stärker die Entwicklungsperspektive eingenommen. So wurden in der externen Studie „Entwicklungsverläufe von pädagogischen Fach- und Lehrkräfte in der frühen MINT-Bildung“ 
pädagogische Fach- und Lehrkräfte, die unterschiedlich lange an dem Programm der Initiative „Haus der kleinen Forscher“ teilgenommen haben, nach ihrem selbsteingeschätzten Kompetenzzuwachs befragt. In der internen Studie wird ein längsschnittlicher Ansatz verfolgt, bei dem pädagogische Fach- und Lehrkräfte vor und zu zwei Zeitpunkten nach einer Fortbildung zu ihrem selbsteingeschätzten Kompetenzzuwachs befragt wurden.

Die Frage nach der Entwicklung durch die letztlich als kontinuierliches Programm angelegten Fortbildungen ist immens wichtig. Erkenntnisse, wie sich pädagogische Fach- und Lehrkräfte unterscheiden oder wie sich Teilnehmerinnen und Teilnehmer, die schon mehrfach an Fortbildungen teilgenommen haben, von solchen unterscheiden, die erst an wenigen Fortbildungen teilgenommen haben, können die Grundlage für z. B. passgenauere Angebote oder möglicherweise stärker aufeinander aufbauende Angebote darstellen.

Ohne die Ergebnisse im Einzelnen vorwegzunehmen, deuten sie auf ein positives Bild der Arbeit der Stiftung „Haus der kleinen Forscher“. Beide Studien zeigen, dass die pädagogischen Fach- und Lehrkräfte ihre eigene Kompetenzentwicklung durch die Teilnahme an den Fortbildungen positiv einschätzen und sie außerdem die Fortbildungsinhalte als relevant einstufen. Unabhängig von den in diesem Band ausführlich diskutierten Einschränkungen durch Selbsteinschätzungen und retrospektiven Befragungen kann dies als wichtige Grundvoraussetzung erfolgreicher Fortbildungen angesehen werden.

Ich möchte an dieser Stelle das Augenmerk auf das fachdidaktische Wissen legen. Als Indikator für (selbsteingeschätztes) fachdidaktisches Wissen wird in den Studien u.a. die Nutzung des Forschungszyklus und seiner verschiedenen Einzelschritte in der Arbeit mit Kindern herangezogen. Der Forschungszyklus, also die (idealisierte) Abfolge verschiedener Schritte bei der Generierung von Erkenntnissen wie beispielsweise fragen/vermuten, beobachten/experimentieren/ überprüfen und interpretieren/schlussfolgern, ist unter verschiedenen Bezeichnungen und mit unterschiedlich ausdifferenzierten Zwischenschritten vor allem aus der entwicklungspsychologischen und naturwissenschaftsdidaktischen Forschung bekannt (Pedaste et al., 2015; Sodian \& Meyer, 2013; White \& Frederiksen, 1998). Er bildet einen zentralen Rahmen für die didaktischen Konzeptionen und Materialien der Stiftung „Haus der kleiner Forscher“. Der Forschungszyklus kann den Lernprozess beim Forschen strukturieren und erfüllt somit eine didaktische Funktion. Viele der Schritte wie das Vermuten, bei dem Vorwissen und Vorerfahrungen aktiviert werden, das Vergleichen von Beobachtungen, das Schlussfolgern aus Beobachtungen, der Rückbezug von Schlussfolgerungen auf die Fragestellung oder Vermutung stehen im engen Zusammenhang mit Merkmalen kognitiv anregender Interaktionen, die als zentral für gelingende Bildungsprozesse gelten. Verschiedene Studien deuten darauf, dass gerade die Begleitung des 
Forschungsprozesses der Kinder in diesem Sinne, also mit kognitiv anregenden und unterstützenden Fragen, Impulsen, Materialien etc., für viele pädagogische Fachkräfte und Lehrkräfte herausfordernd ist (z. B. im Kontext der Naturwissenschaften Leuchter \& Saalbach, 2014; Piasta et al., 2014). Die einzelnen Zitate aus den Interviews zum Forschungszyklus im vorliegenden Band veranschaulichen dieses.

Schaut man sich gleichzeitig die Wünsche und Erwartungen an, die die hier befragten pädagogischen Fach- und Lehrkräfte in Bezug auf die Fortbildungen angeben, zeigt sich, dass eine große Mehrheit neue Themen und direkt umsetzbare Materialien erarbeiten will. Ein deutlich kleinerer Teil der Befragten gibt an, die Begleitung von Kindern oder die Arbeit mit dem Forschungszyklus in den Fortbildungen vertiefen zu wollen. Es gibt also einen (zumindest wahrgenommenen) Widerspruch zwischen Inhalten, die aus der Forschungsperspektive wichtig erscheinen, um naturwissenschaftliche Bildung in Kita und Grundschule langfristig weiterzuentwickeln, und Inhalten, die Teilnehmerinnen und Teilnehmer sich wünschen. Ähnliches ist seit Langem auch aus der Lehrkräftebildung bekannt. Für die Fortbildungen ist es notwendig, beides zu verknüpfen, um einerseits Teilnehmerinnen und Teilnehmer und deren Institutionen nicht zu verlieren und „Commitment“ aufrechtzuerhalten und andererseits die zentralen Elemente von LehrLern-Prozessen stark zu machen. Die langfristige Teilnahme an möglicherweise passgenaueren Fortbildungen mit gezielten Reflexionen über Erfahrungen bei der Umsetzung spezifischer Lehr-Lernumgebungen ist hierfür eine Voraussetzung. Somit sind die Weiterentwicklungen der Fortbildungsangebote der Stiftung sehr plausibel.

Vielleicht könnte auch die Bedeutung des Wissens über den Forschungszyklus oder allgemeiner über die Generierung von naturwissenschaftlichem Wissen ein Ansatzpunkt sein, pädagogische Fach- und Lehrkräfte noch stärker zu unterstützen und zu motivieren, sich mit diesem Teil naturwissenschaftlicher Bildung intensiver auseinanderzusetzen. So ist aus fachdidaktischer Sicht der Forschungszyklus nicht nur als didaktisches Element anzusehen, sondern auch selbst ein wichtiges Ziel naturwissenschaftlicher Bildungsprozesse. Wissen über die Generierung von naturwissenschaftlichem Wissen, über zentrale Denk- und Arbeitsweisen wie das evidenzbasierte Argumentieren und der Umgang mit widersprüchlicher Evidenz sind zentrale Bestandteile naturwissenschaftlicher Bildung, deren Bedeutung uns - gerade jetzt - immer wieder vor Augen geführt wird. 
Für die Entwicklung einer tragfähigen frühen MINT-Bildung ist das Angebot der Stiftung wichtig und damit einhergehend auch die forschungsbasierte Überprüfung und Weiterentwicklung des bestehenden Angebots. Ich freue mich auf weitere Studien und konzeptuelle Weiterentwicklungen rund um das „Haus der kleinen Forscher" und wünsche Ihnen zunächst viele Anregungen beim Lesen dieser Studien!

Prof. Dr. Mirjam Steffensky

Universität Hamburg 


\section{Einleitung}

Stiftung Haus der kleinen Forscher

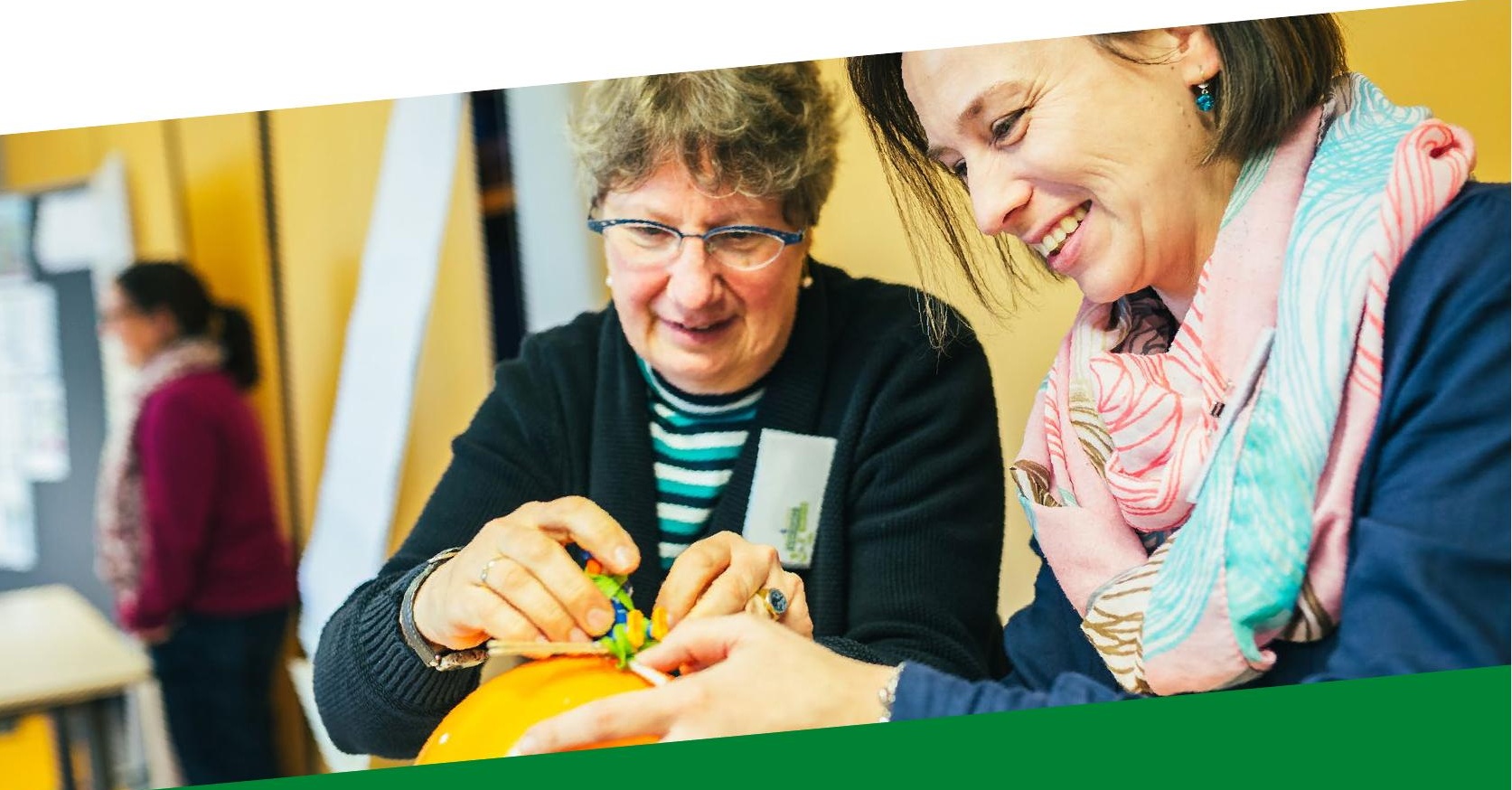

1 Überblick zur Stiftung „Haus der kleinen Forscher“

2 Die Bedeutung der Fort- und Weiterbildung in der frühen (MINT-)Bildung

3 Wirkungen der Bildungsangebote der Initiative „Haus der kleinen Forscher“

4 Hintergründe der externen Studie und stiftungsinternen Befragung zur Professionalisierung von pädagogischen Fach- und Lehrkräften 


\section{1 Überblick zur Stiftung „Haus der kleinen Forscher"}

Die gemeinnützige Stiftung „Haus der kleinen Forscher“ engagiert sich seit 2006 für gute frühe Bildung in den Bereichen Mathematik, Informatik, Naturwissenschaften und Technik (MINT) - mit dem Ziel, Kinder stark für die Zukunft zu machen und zu nachhaltigem Handeln zu befähigen. Gemeinsam mit ihren Netzwerkpartnern vor Ort bietet die Stiftung bundesweit ein Bildungsprogramm an, das pädagogische Fach-, Lehr- und Leitungskräfte dabei unterstützt, Kinder im Kita- und Grundschulalter qualifiziert beim Entdecken, Forschen und Lernen zu begleiten. Das „Haus der kleinen Forscher“ hat zum Ziel, Bildungschancen zu verbessern, das Interesse an MINT und an nachhaltiger Entwicklung zu fördern und dafür pädagogisches Personal zu professionalisieren. Die Bildungsinitiative leistet damit einen wichtigen Beitrag in folgenden Bereichen:

- zur Qualifizierung des frühpädagogischen Personals

- zur Qualitätsentwicklung von Einrichtungen

- zur Persönlichkeits-, Kompetenz und Interessenentwicklung der Kinder

- zur Nachwuchsförderung in den MINT-Bildungsbereichen

Die Hauptaktivitäten der Stiftung sind:

- der Auf- und Ausbau tragfähiger lokaler Netzwerke unter Beteiligung von Akteurinnen und Akteuren vor Ort sowie Beratung und Service für die inzwischen über 200 Netzwerkpartner,

- die Ausbildung von Multiplikatorinnen und Multiplikatoren beziehungsweise Trainerinnen und Trainern, die vor Ort pädagogische Fach-, Lehr- und Leitungskräfte kontinuierlich fortbilden,

- die Entwicklung und Bereitstellung von Fortbildungskonzepten und Materialien für pädagogische Fach-, Lehr- und Leitungskräfte,

- die Unterstützung der Qualitätsentwicklung von Bildungseinrichtungen durch die Zertifizierung als „Haus der kleinen Forscher“ sowie

- die Evaluation und wissenschaftliche Begleitung der Stiftungsaktivitäten. 


\section{Qualifizierungsinitiative für Pädagoginnen und Pädagogen}

Das „Haus der kleinen Forscher“ ist bundesweit die größte Qualifizierungsinitiative für Pädagoginnen und Pädagogen im Bereich der frühen Bildung. Sie unterstützt Kitas, Horte und Grundschulen dabei, mathematische, informatische, naturwissenschaftliche und/oder technische Schwerpunkte zu setzen und Bildung für nachhaltige Entwicklung zu etablieren sowie förderliche Entwicklungs- und Lernumgebungen für Kinder zu schaffen. Der pädagogische Ansatz der Stiftung knüpft an den Ressourcen der Kinder an und betont das gemeinsame Entdecken und Forschen im dialogischen Austausch (Stiftung Haus der kleinen Forscher, 2019b). Die Stiftung fördert mit ihren Aktivitäten zudem die Umsetzung vorhandener Bildungs- und Rahmenlehrpläne der jeweiligen Bundesländer in den Bereichen Mathematik, Informatik, Naturwissenschaften und Technik und unterstützt die Verankerung von BNE in den Bildungsplänen (vgl. u. a. Arnold, Carnap \& Bormann, 2016).

Die inhaltlichen Angebote der Stiftung umfassen neben den Fortbildungen für pädagogische Fach-, Lehr- und Leitungskräfte auch pädagogische Materialien, einen jährlichen Aktionstag sowie Anregungen für Kooperationen:

- Pädagogische Materialien: Für die praktische Umsetzung in den pädagogischen Einrichtungen stellt die Stiftung in den Fortbildungen gedruckt wie online (verfügbar unter: haus-der-kleinen-forscher.de) kostenlos Materialien zur Verfügung, z. B. Themenbroschüren, Forschungs- und Entdeckungskarten, didaktische Materialien und Filmbeispiele.

- Internetpräsenz: Die Webseite haus-der-kleinen-forscher.de bietet Informationen für alle Interessierten, rund um die Bildungsinitiative: darunter das Bildungsangebot, Kontakt zu den lokalen Fortbildungsanbietern, Forscherideen für Kinder, wissenschaftliche Studien oder Broschüren und Bildkarten zum Forschen und Entdecken.

- Campus: Auf der Online-Lernplattform campus.haus-der-kleinen-forscher. de können sich pädagogische Fach-, Lehr- und Leitungskräfte zusätzlich zu den Präsenzfortbildungen vor Ort über offene oder moderierte Online-Kurse fortbilden und untereinander in diversen Foren austauschen. Alle OnlineAngebote der Stiftung stehen kostenfrei zur Verfügung.

- Blog: Der Blog blog.haus-der-kleinen-forscher.de vom „Haus der kleinen Forscher" bietet einen Ort des Austauschs über gute frühe MINT-Bildung für nachhaltige Entwicklung. Hier präsentiert sich die Stiftung als Akteur der nationalen und internationalen Bildungs- und Stiftungswelt und gibt zugleich einen detaillierten Blick hinter die Kulissen vom „Haus der kleinen Forscher“. 
Social-Media-Kanäle: Auf Facebook, Twitter, Instagram und Youtube zeigt die Stiftung unter @kleineForscher (auf Instagram unter kleine_Forscher), wie gute frühe MINT-Bildung aussehen kann. Hier teilt sie neueste Berichte und Studien, Videos von Forscherideen und Aktionen oder informiert über die neusten Fortbildungsangebote aus dem Haus.

\section{f于:}

— Magazin „Forscht mit!“: Pädagogische Fach-, Lehr- und Leitungskräfte erhalten quartalsweise praktische Tipps zum Entdecken und Forschen in der Einrichtung, Informationen zur Arbeit der Stiftung sowie Best-Practice-Berichte aus anderen Einrichtungen und Netzwerken.

- „Tag der kleinen Forscher“: An diesem bundesweiten Mitmachtag können Kinder in ganz Deutschland ein aktuelles Forschungsthema erkunden. Dazu stellt die Stiftung den pädagogischen Einrichtungen Material bereit und ruft Unterstützerinnen und Unterstützer aus Politik, Wirtschaft, Wissenschaft und Gesellschaft zum Mitmachen auf.

- Anregungen zur Kooperation: Interessierte Eltern, Patinnen und Paten sowie andere Bildungspartner unterstützen das gemeinsame Entdecken und Forschen in den Einrichtungen.

- Zertifizierung: Engagierte Einrichtungen werden anhand festgelegter Bewertungskriterien als „Haus der kleinen Forscher“ zertifiziert. Alle sich bewerbenden Einrichtungen erhalten eine detaillierte Rückmeldung mit Anregungen für die weitere Entwicklung des gemeinsamen Entdeckens und Forschens mit den Kindern.

- Kinder-Webseite: Unter meine-forscherwelt.de gelangen Kinder im Grundschulalter in einen interaktiven Forschergarten, der sie zu eigenständigen Entdeckungsreisen animiert. Für Pädagoginnen und Pädagogen stehen Tipps für die Lernbegleitung zur Verfügung.

- Service-Portal Integration: Unter integration.haus-der-kleinen-forscher.de erhalten Fach- und Lehrkräfte durch vielfältige Materialien, praxisnahe Impulse sowie inspirierenden Erfahrungsaustausch Unterstützung bei der Integration geflüchteter Kinder in Kita, Hort und Grundschule.

\section{Bundesweite Vernetzung}

Das „Haus der kleinen Forscher“ lebt als bundesweite Bildungsinitiative vom Engagement vielfältiger Akteurinnen und Akteure vor Ort - den lokalen Netzwerken, die als dauerhafte Partner und Fortbildungsanbieter in den Regionen agieren. 
Zu den derzeit 213 Netzwerkpartnern zählen Kommunen und Kita-Träger, Wirtschaftsverbände, Science-Center, Museen, Unternehmen, Stiftungen, Vereine usw. Seit 2011 steht das Fortbildungsprogramm der Initiative auch Horten und Ganztagsgrundschulen offen.

Circa 80.000 pädagogische Fach- und Lehrkräfte aus über 32.600 Kitas, Horten und Grundschulen haben bereits am Fortbildungsprogramm der Initiative teilgenommen, davon pädagogische Fachkräfte aus rund 26.300 Kitas sowie Fach- und Lehrkräfte aus mehr als 1.500 Horten und über 4.800 (Ganztags-) Grundschulen.

Deutschlandweit sind rund 5.400 Kitas, Horte und Grundschulen als „Haus der kleinen Forscher“ zertifiziert, darunter über 4.900 Kitas. Seit Herbst 2013 können sich auch Horte und Grundschulen zertifizieren lassen. Seitdem haben rund 200 Horte und rund 300 Grundschulen das Zertifikat „Haus der kleinen Forscher" erhalten (Stand Dezember 2019).

\section{Das kontinuierliche Fortbildungsprogramm}

Die Stiftung „Haus der kleinen Forscher“ konzentriert sich auf die Weiterqualifizierung von Pädagoginnen und Pädagogen in Hinblick auf das Entdecken und Erforschen mathematischer, informatischer, naturwissenschaftlicher und/oder technischer Themen mit Kindern. Seit 2018 gibt es auch Weiterbildungsangebote mit dem Fokus Bildung für nachhaltige Entwicklung. Das Ziel ist eine kontinuierliche Begleitung der pädagogischen Fach-, Lehr- und Leitungskräfte: Die Teilnahme an Fortbildungen zu unterschiedlichen Themen erweitert sukzessive das methodische Repertoire und vertieft das Verständnis des pädagogischen Ansatzes der Stiftung. Im Wechsel von Präsenzfortbildungen und Transferphasen können die Pädagoginnen und Pädagogen das Gelernte in der Praxis ausprobieren und sich dazu in der nächsten Fortbildung austauschen. Zusätzlich stellt die Stiftung ein ständig wachsendes Online-Angebot zur Verfügung, mit dem jede beziehungsweise jeder Interessierte individuell, flexibel und kostenfrei die Fortbildungsinhalte auffrischen oder vertiefen kann. Dazu gehören sowohl offene Online-Kurse, die zu jederzeit selbstständig bearbeitet werden können, als auch moderierte OnlineKurse zu festen Zeiten. Hier werden die Inhalte gemeinsam mit anderen Teilnehmenden erarbeitet, während eine Moderatorin beziehungsweise ein Moderator sie begleitet. Webbasierte Seminare (kurz: Webinare) finden zu einem bestimmten Termin statt und beinhalten einen interaktiv gestalteten Online-Vortrag. Um die eigenen Praxiserfahrungen gemeinsam mit anderen pädagogischen Fach- und Lehrkräften zu reflektieren, gibt es zudem themenspezifische Foren, die zum Austausch von praktischen Erfahrungen genutzt werden können.

Um möglichst vielen interessierten pädagogischen Fach-, Lehr- und Leitungskräften die Teilnahme an Fortbildungen zu ermöglichen, findet die Weiterqualifi- 
zierung über ein Multiplikationsmodell statt: Die Stiftung „Haus der kleinen Forscher" bildet an mehreren Standorten in Deutschland Trainerinnen und Trainer aus, die ihrerseits Fortbildungen für Pädagoginnen und Pädagogen in ihrem lokalen Netzwerk durchführen (Stiftung Haus der kleinen Forscher, 2019c). Die Trainerinnen und Trainer qualifizieren sich durch die Teilnahme an den Präsenz- und Online-Fort-

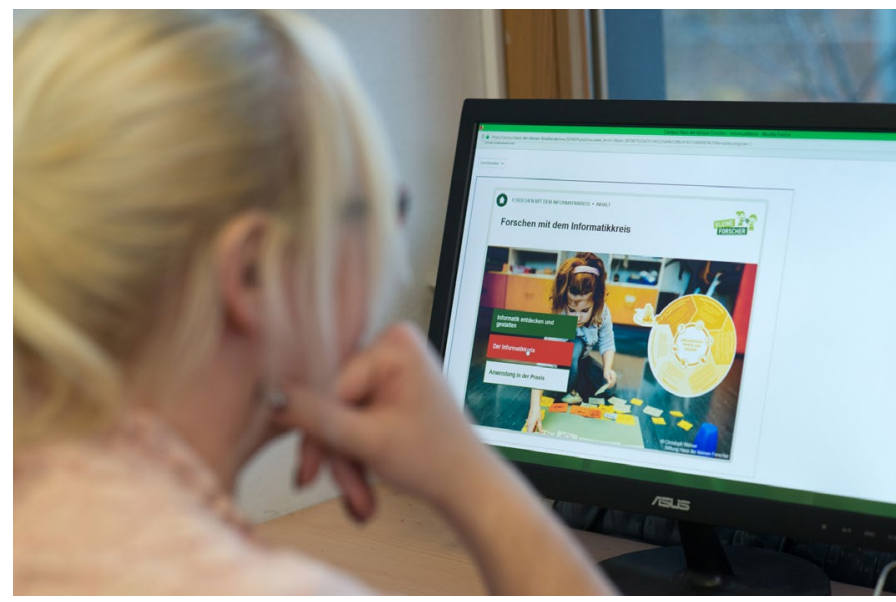
bildungen der Stiftung dafür, Fortbildungen mit Pädagoginnen und Pädagogen durchzuführen. Als Unterstützung erhalten sie ausführliche Arbeitsunterlagen für ihre Aufgabe in der Erwachsenenbildung sowie die Möglichkeit, persönliches Feedback im Hospitationsprogramm der Stiftung oder in Form von Videocoaching zu bekommen. Für die Auffrischung und Vertiefung der Fortbildungsinhalte steht zudem der Online-Campus für Trainerinnen und Trainer zur Verfügung. Die digitale Lernplattform bietet neben einer Vielzahl von Online-Lernangeboten auch inhaltliche Informationen und Arbeitsunterlagen zu den einzelnen Fortbildungsmodulen. $\mathrm{Zu}$ bestimmten Themen gibt es die Möglichkeit, eigenständig offene E-LearningModule zu bearbeiten, an tutoriell begleiteten Kursen teilzunehmen sowie die Online-Begleitkurse zu Präsenzfortbildungen zu nutzen. Darüber hinaus können die Trainerinnen und Trainer in Themenforen oder offenen Chats miteinander in Kontakt treten und sich austauschen. Für die Trainerinnen und Trainer werden in der Bildungsinitiative jedes Jahr unterschiedliche Fortbildungsthemen angeboten.

Seit 2017 ist der Einstieg in das Bildungsangebot der Stiftung sowohl für pädagogische Fach-, Lehr- und Leitungskräfte als auch für Trainerinnen und Trainer flexibel gestaltet. ${ }^{1}$ Sieht die Lernbegleitung noch Entwicklungsbedarf in ihrer pädagogischen Kompetenz beziehungsweise möchte sich einen Überblick zum

1 Mit der Flexibilisierung des Einstiegs in das Bildungsprogramm der Stiftung nimmt die Stiftung ihre Zielgruppen in eine größere Eigenverantwortung. Gemäß dem Menschenbild eigenaktiv Lernender, das dem pädagogischen Konzept der Stiftung zugrunde liegt, setzt sie darauf, dass die pädagogischen Fach-, Lehr- und Leitungskräfte sowie die Trainerinnen und Trainer selbst erkennen können, wo sie mit ihren Interessen und Bedarfen stehen und welches Thema oder Format der für sie passende Einstieg ins Bildungsprogramm des „Hauses der kleinen Forscher“ ist beziehungsweise welches Angebot sie für ihre weitere Professionalitätsentwicklung nutzen wollen. Um die Nutzerinnen und Nutzer bestmöglich in ihrer Professionalisierung als Lernbegleitung zu unterstützen, bietet die Stiftung gezielt Bildungsangebote an und entwickelt diese kontinuierlich wirkungs- und bedarfsorientiert weiter. 
pädagogischen Konzept der Stiftung verschaffen, so erhält sie das Angebot, mit den Präsenzfortbildungen „Forschen mit Wasser“ oder „Forschen mit Luft“ einzusteigen, in denen der pädagogische Ansatz der Stiftung ausführlich thematisiert wird, beziehungsweise das Seminar oder den Online-Kurs „Grundlagenseminar Der pädagogische Ansatz der Stiftung ,Haus der kleinen Forscher““ zu besuchen. Ebenso können die pädagogischen Fach-, Lehr- und Leitungskräfte beziehungsweise die Trainerinnen und Trainer als Einstieg ein anderes Modul zu mathematischen, informatischen, naturwissenschaftlichen, technischen Themen oder zur Bildung für nachhaltige Entwicklung wählen. Die Inhalte werden in verschiedenen Formaten angeboten: Fortbildungen vor Ort, Selbstbildungsformate (wie OnlineKurse oder gedruckte pädagogische Materialien) und Bildungsveranstaltungen. Das Zertifikat „Haus der kleinen Forscher“ unterstützt darüber hinaus bei der Qualitätsentwicklung der pädagogischen Arbeit in den Einrichtungen und macht das Engagement für gute frühe MINT-Bildung nach außen sichtbar. Die Stiftung orientiert sich dabei stark an den Bedarfen, dem Vorwissen, den Vorerfahrungen und Interessen ihrer Zielgruppen.

Der Ansatz einer möglichst individuellen und bedarfsgerechten Unterstützung, den die Stiftung auf Ebene der Kinder wie der pädagogischen Fach-, Lehrund Leitungskräfte verfolgt, findet durch das Qualitätssystem für Fortbildung auch auf der Ebene der Trainerinnen und Trainer Anwendung. Wesentliche Elemente des Systems betreffen die Bewerbung und Akkreditierung zu Beginn der Tätigkeit als Trainerin beziehungsweise Trainer, bedarfsgerecht gestaltete Qualifizierungsphasen sowie eine alle zwei Jahre wiederkehrende Re-Akkreditierung. Es systematisiert und erweitert auf Basis der Zieldimensionen für Multiplikatorinnen und Multiplikatoren früher MINT-Bildung die Anforderungen an Trainerinnen und Trainer und macht diese explizit (Stiftung Haus der kleinen Forscher, 2019c).

Auch die inhaltlichen Angebote der Stiftung für pädagogische Fach-, Lehrund Leitungskräfte werden auf der Grundlage fachlich fundierter Zieldimensionen entwickelt. Sie spezifizieren, welche Ziele mit bestimmten Angeboten erreicht werden sollen. Sowohl zu den einzelnen MINT-Disziplinen als auch zur Bildung für nachhaltige Entwicklung wurden gemeinsam mit Fachexpertinnen und -experten Zieldimensionen für Kinder und pädagogische Fach- und Lehrkräfte erarbeitet und als Orientierungsgrundlage zur Angebotsentwicklung genutzt (Stiftung Haus der kleinen Forscher, 2013, 2015, 2017a, 2018a, 2019a, 2019c).

Mit den Fortbildungen zur Bildung für nachhaltige Entwicklung, die die Stiftung seit 2018 anbietet, richtet sie sich erstmals explizit auch an die Leitungskräfte pädagogischer Einrichtungen mit einer eigens für diese Zielgruppe konzipierten Fortbildung. Entsprechend wurden die Zieldimensionen einer Bildung für nachhaltige Entwicklung auch für die Ebene der Leitungskräfte erarbeitet (Stiftung Haus der kleinen Forscher, 2019a). 
Das Stiftungsangebot wurde zuletzt mit der Fortbildung „MINT ist überall“ um ein Bildungsangebot ergänzt, das alle vier MINT-Disziplinen gemeinsam thematisiert (siehe Fazit und Ausblick, in diesem Band).

\section{Wissenschaftliche Begleitung und Qualitätsentwicklung}

Alle Aktivitäten der Bildungsinitiative werden kontinuierlich wissenschaftlich begleitet und evaluiert. Die Stiftung „Haus der kleinen Forscher“ pflegt einen offenen Austausch mit Wissenschaft und Fachpraxis und versteht sich als lernende Organisation.

Ein umfangreiches Spektrum an Maßnahmen dient der Sicherung und Weiterentwicklung der Qualität im „Haus der kleinen Forscher“(siehe Abbildung 1). Das stiftungsinterne Qualitätsmanagement erfasst mit eigenen Evaluationsmaßnahmen und umfassendem Monitoring alle wichtigen Aktivitäten und Angebote. Dafür nutzt die Stiftung eine ganze Reihe an Datenquellen (wie z. B. anlassbezogene Befragungen der Netzwerkkoordinatorinnen und koordinatoren, der Trainerinnen und Trainer sowie der pädagogischen Fach-, Lehr- und Leitungskräfte); eine Kombination aus quer- und längsschnittlichen Daten ermöglicht den Blick auf die aktuelle Situation und auch auf wichtige Veränderungen in den letzten Jahren. Um auf die Erkenntnisbedarfe der Stiftung flexibel reagieren zu können, werden mehrere Erhebungen mit verschiedenen Zielgruppen zu unterschiedlichen Zeitpunkten durchgeführt.

Die längsschnittliche Perspektive spielt in den internen Evaluations- und Monitoring-Maßnahmen der Stiftung eine zunehmend wichtigere Rolle, auch um dem Anspruch einer stärkeren Wirkungsorientierung gerecht zu werden. Mit dem regelmäßig erscheinenden Monitoring-Bericht stellt die Stiftung wichtige Ergebnisse dieser Maßnahmen bereit. So beschreibt der Monitoring-Bericht 2016/2017 auf Grundlage einer Wirkungskette, wie das Fortbildungsangebot der Initiative zur Verbesserung der frühen MINT-Bildung in Deutschland beiträgt (Stiftung Haus der kleinen Forscher, 2017b). Im Monitoring-Bericht 2018/2019 werden die querschnittlichen Analysen fortgeführt und durch Untersuchungsergebnisse im Längsschnitt methodisch ergänzt (Stiftung Haus der kleinen Forscher, 2020).

Die inhaltliche (Weiter-)Entwicklung neuer Stiftungsangebote erfolgt stets fachlich fundiert und in Kollaboration mit Wissenschaft und Praxis; neue Stiftungsangebote werden gemeinsam und im Austausch mit der Praxis entwickelt und getestet. In Zusammenarbeit mit Pädagoginnen und Pädagogen aus Kitas sowie aus Horten und Grundschulen findet für jedes neue Fortbildungsangebot eine ausführliche Pilotierung statt, bevor die Fortbildungskonzepte und Materialien in den regionalen Netzwerken verbreitet werden. Dabei prüfen die mitwirkenden pädagogischen Fach-, Lehr- und Leitungskräfte erste Praxisideen auf ihre Umsetzbarkeit und geben Feedback zu den Unterstützungsangeboten der Stiftung. Die 
Fortbildungskonzepte werden auf Basis dieser Rückmeldungen überarbeitet und weiterentwickelt.

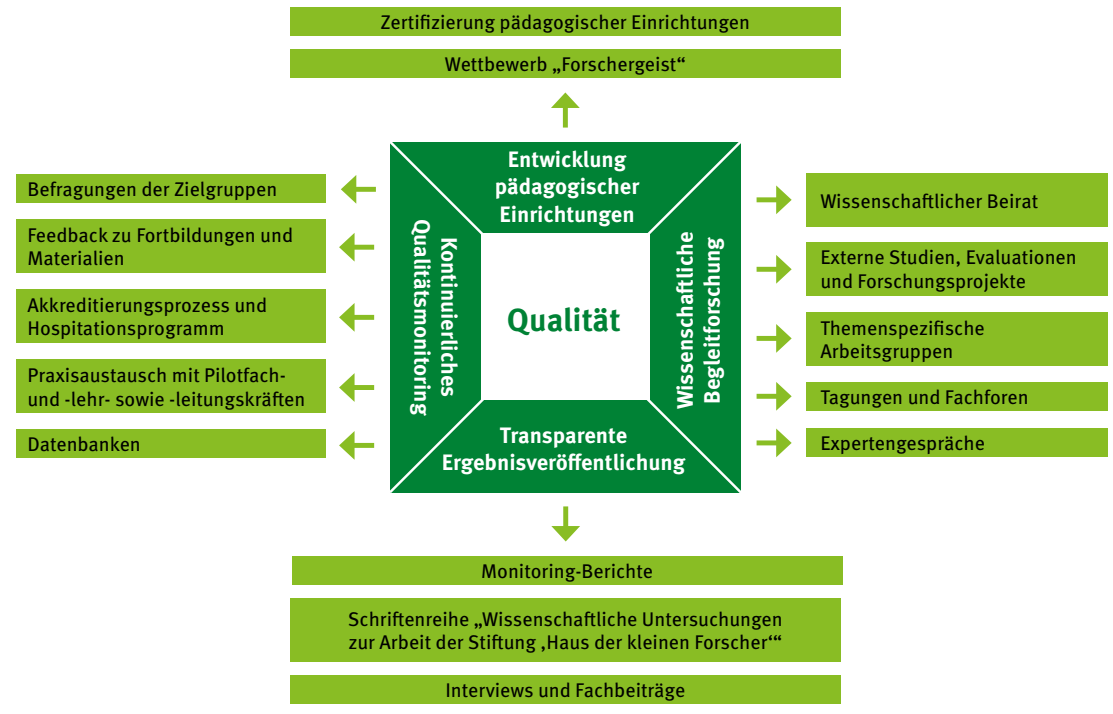

Abbildung 1. Übersicht der Maßnahmen zu Sicherung und Weiterentwicklung der Qualität der Stiftungsarbeit

Auf Einrichtungsebene ist die Zertifizierung als „Haus der kleinen Forscher“ ein weiteres wichtiges Instrument der Qualitätsentwicklung (Stiftung Haus der kleinen Forscher, 2017c). Über die Vergabe des Zertifikats entscheidet die Stiftung in einem standardisierten Verfahren, das in Anlehnung an das Deutsche Kindergarten Gütesiegel und unter Beteiligung eines Teams aus Wissenschaftlerinnen und Wissenschaftlern (Yvonne Anders, Christa Preissing, Ursula Rabe-Kleberg, Jörg Ramseger und Wolfgang Tietze) entwickelt wurde. Die Reliabilität und Validität des Zertifizierungsverfahrens für Kitas wurden in einer externen wissenschaftlichen Studie bestätigt (Anders \& Ballaschk, 2014). Die Zertifizierung als „Haus der kleinen Forscher“ ist ein kostenfreies Verfahren zur Erfassung und Steigerung der pädagogischen Qualität bei der Umsetzung von MINT-Bildungsinhalten. Durch die Beantwortung der Fragen im Zertifizierungsfragebogen und die darauffolgende ausführliche Rückmeldung der Stiftung mit praktischen Anregungen und Tipps werden Kitas, Horte und Grundschulen in ihrer Qualitätsentwicklung unterstützt. Eine Folgezertifizierung kann alle zwei Jahre erfolgen und ermöglicht die langfristige Verankerung und Weiterentwicklung der Bildungsqualität auf Einrichtungsebene.

Mit dem bundesweiten Kita-Wettbewerb „Forschergeist“ möchten die Deutsche Telekom Stiftung und die Stiftung „Haus der kleinen Forscher“ das Engage- 
ment der Kita-Fachkräfte sowie die Qualität der Einrichtungen würdigen und sie weiter zur frühpädagogischen Bildungsarbeit motivieren. Im September 2019 riefen die Initiatoren den „Forschergeist“-Wettbewerb bereits zum fünften Mal aus. Gesucht und in 2020 prämiert wurden herausragende Projekte, die Kinder für die Welt der Mathematik, Informatik, Naturwissenschaften und Technik sowie einer Bildung für nachhaltige Entwicklung begeistert haben. Die prämierten Projekte werden dokumentiert und veröffentlicht, damit sie als gute Beispiele auch andere Fachkräfte für das Forschen und Entdecken in der Kita begeistern (Deutsche Telekom Stiftung \& Stiftung Haus der kleinen Forscher, in Vorb.). ${ }^{2}$

Neben einem kontinuierlichen Monitoring zu Zwecken der Qualitätssicherung und der Qualitätsentwicklung wird die Stiftungsarbeit im Rahmen einer langfristig angelegten externen Begleitforschung mit renommierten Partnern fachlich fundiert und in Forschungsprojekten evaluiert. Zwei unabhängige Forschungsgruppen untersuchten von 2013 bis 2017 die naturwissenschaftlichen Bildungswirkungen in der frühen Kindheit (Stiftung Haus der kleinen Forscher, 2018b). Ziel des ersten Forschungsprojekts „Early Steps into Science“ (kurz: EASI Science, gefördert von der Stiftung „Haus der kleinen Forscher“ und dem Bundesministerium für Bildung und Forschung) war es, Erkenntnisse über Wirkungen früher naturwissenschaftlicher Bildungsangebote auf naturwissenschaftliche Kompetenzen von pädagogischen Fachkräften und Kindern in Kitas zu gewinnen. Die Ergebnisse zeigen, dass pädagogische Fachkräfte mit naturwissenschaftlichen Fortbildungen über ein höheres Fachwissen und mehr fachdidaktische Kenntnisse verfügen als eine Vergleichsgruppe ohne Fortbildungen. Zudem sind die Motivation zu und das Interesse an naturwissenschaftlicher Bildung fortgebildeter Pädagoginnen und Pädagogen größer. Auch die Kinder zeigen mehr Lernfreude, Interesse an Naturwissenschaften sowie Selbstvertrauen in ihr eigenes Können, wenn ihre Kita einen naturwissenschaftlichen Schwerpunkt hat (Steffensky et al., 2018). Das zweite Forschungsprojekt „Early Steps into Science and Literacy“ (kurz: EASI ScienceL, gefördert von der Stiftung „Haus der kleinen Forscher“, der Baden-Württemberg Stiftung und der Siemens Stiftung) untersuchte sprachliche Bildungswirkungen und die Interaktionsqualität im Kontext naturwissenschaftlicher Bildungsangebote. In der Studie konnte gezeigt werden, dass sich forschendes Lernen gut für die Sprachbildung eignet. Fachkräfte mit naturwissenschaftlichen Fortbildungen gestalten sprachlich anregendere Lerngelegenheiten für Kinder als Fachkräfte ohne Fortbildungen in diesem Bereich. Die sprachlichen Fähigkeiten der Kinder sind größer, wenn die sie betreuenden Fachkräfte zuvor eine kombinierte Fortbildung zu Naturwissenschaften und Sprache der Stiftung „Haus der kleinen Forscher“

2 Die Dokumentation der prämierten Projekte beim Forschergeist 2012, 2014, 2016, 2018 und 2020 sind zu finden unter forschergeist-wettbewerb.de. 
besucht haben. Zudem zeigen sich positive Zusammenhänge zwischen der durch die Fachkraft gestalteten naturwissenschaftsbezogenen Prozessqualität und den naturwissenschaftlichen Kompetenzen der Kinder (Rank et al., 2018).

Seit 2020 fördert die Stiftung gemeinsam mit dem Bundesministerium für Bildung und Forschung eine Studie zu den Wirkungen des Modellprogramms „KiQ - gemeinsam für Kita-Qualität. Wenn Entdecken und Forschen zum Alltag werden“. Mit diesem Kita-Programm nimmt die Stiftung die Einrichtungen als ein Gesamtsystem in den Blick und setzt auf einen Weiterbildungsansatz, der personenbezogene und organisationale Aspekte miteinander verschränkt und berücksichtigt, dass sich die Art der Führung und Organisation einer Einrichtung auf die pädagogische Arbeit in dieser Einrichtung auswirkt (Deutsches Jugendinstitut \& Weiterbildungsinitiative Frühpädagogische Fachkräfte, 2014; Strehmel \& Ulber, 2017). Während der Modellphase (2020-2022) wird das Programm „KiQ“ sowohl von der Stiftung evaluiert als auch von einer externen Forschungsgruppe wissenschaftlich begleitet. Ziel ist es, wichtige Erkenntnisse hinsichtlich der Erfolgsindikatoren für eine Implementierung des entdeckenden und forschenden Lernens in Kitas zu gewinnen. Weiterhin sollen Empfehlungen zu einer zukünftigen Verankerung des Konzepts im regelmäßigen Qualifizierungsangebot der Stiftung und ihrer gleichnamigen Fortbildungsinitiative „Haus der kleinen Forscher“ abgeleitet werden.

Die Ergebnisse der wissenschaftlichen Begleitung veröffentlicht die Stiftung transparent in der wissenschaftlichen Schriftenreihe; alle Publikationen sind zudem über die Webseite frei verfügbar. ${ }^{3}$

Ein Wissenschaftlicher Beirat berät die Stiftung zu Forschungsfragen sowie zur fachlichen Fundierung des Stiftungsangebots. Er setzt sich aus unabhängigen Wissenschaftlerinnen und Wissenschaftlern unterschiedlicher Fachgebiete zusammen und spricht Empfehlungen an den Vorstand und den Stiftungsrat aus. Die Mitglieder des Beirats sind hochkarätige Expertinnen und Experten relevanter Fachdisziplinen und werden jeweils für drei Jahre berufen. Von 2018 bis 2020 sind dies die folgenden Mitglieder:

- Vorsitz: Prof. Dr. Hans-Günther Roßbach, Universität Bamberg, Lehrstuhl für Elementar- und Familienpädagogik

- Prof. Dr. Fabienne Becker-Stoll, Staatsinstitut für Frühpädagogik (IFP), München

3 Alle Ergebnisse und Publikationen zur wissenschaftlichen Begleitung sind als PDF verfügbar unter: www.haus-der-kleinen-forscher.de, Rubrik „Wissenschaftliche Begleitung“. Alle Ergebnisse der externen Begleitforschung werden zudem in der vorliegenden wissenschaftlichen Schriftenreihe veröffentlicht. Eine Übersicht der bisher erschienenen Bände befindet sich auf www.haus-der-kleinenforscher.de beziehungsweise am Ende dieses Bands. 
- Prof. Dr. Marcus Hasselhorn, Deutsches Institut für Internationale Pädagogische Forschung (DIPF), Frankfurt am Main, Abteilung Bildung und Entwicklung / Prof. Dr. Jan Lonnemann, Universität Potsdam, Lehrstuhl für Empirische Kindheitsforschung

- Prof. Dr. Christoph Igel, Technische Universität Chemnitz, Bildungstechnologie

- Prof. Dr. Bernhard Kalicki, Deutsches Jugendinstitut e. V. (DJI), München, Abteilung Kinder und Kinderbetreuung, und Evangelische Hochschule Dresden, Lehrstuhl für Frühkindliche Bildung

- Prof. Dr. Alexander Kauertz, Universität Koblenz-Landau, Lehrstuhl für Physikdidaktik und Techniklehre

- Prof. Dr. Armin Lude, Pädagogische Hochschule Ludwigsburg, Abteilung Biologie, Schwerpunkt Bildung für nachhaltige Entwicklung

- Prof. Dr. Johannes Magenheim, Universität Paderborn, Lehrstuhl für Didaktik der Informatik

- Prof. Dr. Jörg Ramseger, Freie Universität Berlin, Arbeitsstelle Bildungsforschung Primarstufe

- Prof. Pia S. Schober, Ph.D, Universität Tübingen, Lehrstuhl für Soziologie mit Schwerpunkt Mikrosoziologie, und Deutsches Institut für Wirtschaftsforschung (DIW Berlin), Abteilung Bildung und Familie

- Prof. Dr. Mirjam Steffensky, Universität Hamburg, Fakultät für Erziehungswissenschaft - Didaktik der Chemie

- Prof. Dr. Wolfgang Tietze, PädQUIS gGmbH, An-Institut der Alice Salomon Hochschule, Berlin / Prof. Dr. Catherine Walter-Laager, Universität Graz, Arbeitsbereich Elementarpädagogik

- Prof. Dr. Christian Wiesmüller, Pädagogische Hochschule Karlsruhe, Abteilung für Physik und Technische Bildung und Deutsche Gesellschaft für Technische Bildung (DGTB), Ansbach

- Prof. Dr. Bernd Wollring, Universität Kassel, Lehrstuhl für Didaktik der Mathematik 


\section{Die Bedeutung der Fort- und Weiterbildung in der frühen (MINT-)Bildung}

Der frühen Bildung wird gesellschaftlich große Bedeutung zugeschrieben. Auch in den Bildungsbereichen Mathematik, Informatik, Naturwissenschaften und Technik entwickeln viele Einrichtungen der frühen Bildung ihre pädagogische Arbeit weiter. Pädagogische Fach- und Lehrkräfte sind diejenigen, die im direkten Umgang mit den Kindern diese Arbeit in der Einrichtung umsetzen und sich dafür durch Fortbildungsangebote (weiter-)qualifizieren (Buschle \& Gruber, 2018; Stiftung Haus der kleinen Forscher, 2019c). Insgesamt ist das Arbeitsfeld der frühen Bildung und der Kindertageseinrichtungen im Speziellen in den letzten Jahren durch dynamische Transformationsprozesse geprägt (u. a. quantitativer Ausbau der Kindertagesbetreuung, Einführung länderspezifischer Bildungspläne, wachsende Bedeutung einer Bildung für nachhaltige Entwicklung; vgl. u.a. Starke, 2017), was zu einem veränderten und gewachsenen Anspruch an die Professionalisierung der pädagogischen Fach- und Lehrkräfte führt (vgl. Buschle \& Gruber, 2018; Hippel, 2011). Hier nimmt die Fort- und Weiterbildung im Professionalisierungsprozess der Pädagoginnen und Pädagogen eine bedeutende Rolle ein (Stiftung Haus der kleinen Forscher, 2019c). Buschle und Gruber sehen darin die zentrale Funktion der beruflichen Weiterbildung im Arbeitsfeld der Kindertageseinrichtungen: „Weiterbildung soll der Spezialisierung der Fachkräfte über die breit angelegte Ausbildung hinaus dienen, aber auch der adäquaten und schnellen Begegnung aktueller Herausforderungen und gesellschaftspolitischer Veränderungen“ (2018, S. 10). Die von ihnen durchgeführte Studie der Weiterbildungsinitiative Frühpädagogischer Fachkräfte (WiFF) geht dem Stellenwert der Weiterbildung im Arbeitsfeld der Kindertageseinrichtungen nach.

Die Studie zeigt, dass sich pädagogische Fachkräfte in der frühen Bildung im Vergleich zu anderen Berufsgruppen überdurchschnittlich an Weiterbildung beteiligen (vgl. auch Hippel, 2011; König \& Friederich, 2015). Die berufliche Weiterbildung ist Teil des „Berufsethos“ frühpädagogischer Fachkräfte. Sie begreifen „Weiterbildungsteilnahmen als Teil ihrer beruflichen Identität sowie als Bedingung für die eigene berufliche Weiterentwicklung (Buschle \& Gruber, 2018).

Insgesamt zeigt die Studie eine hohe Bedeutung der beruflichen Weiterbildung für die Professionalisierung pädagogischer Fachkräfte, weist jedoch auf strukturelle Barrieren hin, die sowohl eine Weiterbildungsteilnahme als auch die Umsetzung der Fortbildungsinhalte in der pädagogischen Praxis erschweren. Ein fokussierter Blick auf Spezifika der MINT-bezogenen Professionalisierung pädagogischer Fach- und Lehrkräfte, auf damit verbundene Bedarfe und auf deren Ver- 
änderung im Verlauf des Professionalisierungsprozesses fehlt bisher (Nationales MINT Forum, 2018).

\section{Bildungsangebote der Stiftung „Haus der kleinen Forscher“}

Pädagoginnen und Pädagogen können Kindern Erfahrungen ermöglichen, die ihnen helfen, ihre Kompetenzen zu erweitern, eigene Antworten zu finden und Selbstwirksamkeit zu spüren (Stiftung Haus der kleinen Forscher, 2019b). Die Angebote der Stiftung „Haus der kleinen Forscher“ unterstützen pädagogische Fachund Lehrkräfte dabei, Begeisterung für das gemeinsame Entdecken und Forschen mit Kindern zu entwickeln, ihre Möglichkeiten zur pädagogischen Unterstützung zu erweitern, ihr fachdidaktisches Wissen und Handeln in Bezug auf frühe MINTBildung für nachhaltige Entwicklung auszubauen, ihre wissenschaftlichen Denkund Arbeitsweisen auszuweiten sowie ihr professionelles Rollen- und Selbstverständnis zu reflektieren (vgl. Stiftung Haus der kleinen Forscher, 2013, 2015, 2017a, 2018a, 2019a). Nimmt eine Pädagogin beziehungsweise ein Pädagoge zum ersten Mal an einer „Haus der kleinen Forscher“-Fortbildung teil, beginnt ein persönlicher Entwicklungsprozess, der im weiteren Verlauf der Teilnahme am Bildungsprogramm seine Wirkungen in der pädagogischen Arbeit entfalten kann (siehe auch Stiftung Haus der kleinen Forscher, 2020). Das vielfältige Bildungsangebot der Stiftung ermöglicht es den Pädagoginnen und Pädagogen, kontinuierlich ihr pädagogisches Repertoire zu erweitern. Zur Auffrischung und Vertiefung der Fortbildungsinhalte stellt die Stiftung kostenfreie Materialien, offene sowie moderierte Online-Kurse und eine digitale Austauschplattform zur Verfügung.

Das „Haus der kleinen Forscher“ unterstützt auf diese Weise pädagogische Fach-, Lehr- und Leitungskräfte bei ihrer Professionalisierung und trägt dadurch neben der individuellen Professionalisierung zur Qualitätsentwicklung von Einrichtungen sowie zur Persönlichkeits- und Interessenentwicklung der Kinder bei. Beispielsweise wird Weiterbildung von Kommunen, Länder- und Bundesebene sowie Trägern genutzt, um aktuelle Entwicklungen im Arbeitsfeld der Kindertageseinrichtungen zu etablieren. Insbesondere kompetenzorientierte Weiterbildung knüpft an Handlungskompetenzen, wie die Art und Weise mit der Kindern Lerngelegenheiten geboten werden, an und zahlt damit auf die pädagogische Qualität in den Einrichtungen ein. Eine viel diskutierte Frage ist in diesem Zusammenhang, wie Fort- und Weiterbildungen im Bereich der frühen Bildung beschaffen sein müssen, um tatsächlich die Qualität gesamter Kindertageseinrichtungen nachhaltig weiterzuentwickeln (vgl. u. a. Friederich, 2019; König \& Friederich, 2015). 


\section{Wirkungen der Bildungsangebote der Initiative „Haus der kleinen Forscher“}

Bereits seit einigen Jahren arbeitet die Stiftung „Haus der kleinen Forscher“verstärkt wirkungsorientiert. Eine in diesem Zusammenhang entwickelte Wirkungskette (siehe Abbildung 2) für die Zielgruppe der Pädagoginnen und Pädagogen beschreibt, wie das Fortbildungsangebot der Initiative zur Verbesserung der frühen MINT-Bildung in Deutschland beitragen möchte (Stiftung Haus der kleinen Forscher, 2017b, 2020).
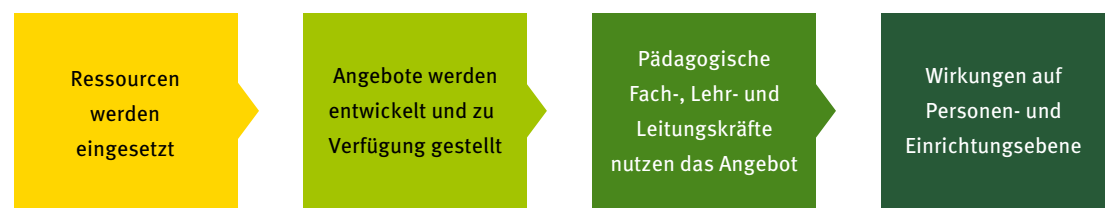

Abbildung 2. Vereinfachte Wirkungskette der Initiative "Haus der kleinen Forscher" in Hinblick auf die Zielgruppe der pädagogischen Fach-, Lehr- und Leitungskräfte sowie auf die Einrichtungen (Stiftung Haus der kleinen Forscher, 2020)

Mit der Wirkungskette wurden sowohl entscheidende Wirkungsziele identifiziert als auch die zu deren Erreichung notwendigen Schritte benannt:

\section{Einsatz von Ressourcen}

Die Ressourcen der Bildungsinitiative „Haus der kleinen Forscher“ umfassen die Mittel, die eingesetzt werden, um ihre Arbeit umsetzen zu können. Dazu gehören sowohl die lokalen Netzwerkpartner mit den Koordinatorinnen und Koordinatoren sowie den Trainerinnen und Trainern als auch die Mitarbeiterinnen und Mitarbeiter der Stiftung. Zudem beinhaltet dies die investierten Mittel durch Stiftung, Netzwerkpartner und die weiteren Unterstützerinnen und Unterstützer der Bildungsinitiative.

\section{Entwicklung und Bereitstellung von Angeboten}

Mit dem Einsatz der Ressourcen können die verschiedenen Fortbildungsformate des Bildungsangebots der Initiative gemeinsam mit Expertinnen und Experten fachlich fundiert, (weiter-)entwickelt sowie Trainerinnen und Trainer qualifiziert werden. Die Fortbildungsangebote werden über die lokalen Netzwerkpartner und als Online-Fortbildungen über die Webseite der Stiftung deutschlandweit zur Verfügung gestellt. Da es der Stiftung ein besonderes Anliegen ist, dass die päda- 
gogischen Fach-, Lehr- und Leitungskräfte das Fortbildungsangebot fortlaufend nutzen, wird es kontinuierlich ausgebaut und erweitert.

\section{Pädagogische Fach-, Lehr- und Leitungskräfte nutzen das Angebot}

Pädagogische Fach-, Lehr- und Leitungskräfte in ganz Deutschland nutzen das Fortbildungsangebot und die Materialien der Initiative „Haus der kleinen Forscher“. Stiftungseigene Monitoring-Daten weisen darauf hin, dass die durchschnittliche Anzahl der besuchten Fortbildungen sukzessive mit der Teilnahmedauer der pädagogischen Fach-, Lehr- und Leitungskräfte steigt. Im Durchschnitt besuchen die teilnehmenden Pädagoginnen und Pädagogen etwa eine (0.9) Fortbildung der Initiative „Haus der kleinen Forscher“ pro Jahr. Bei einer Teilnahme von einem bis unter zwei Jahren liegt die durchschnittliche Anzahl bei zwei Fortbildungen (1.7). Diejenigen, die bereits seit sieben bis unter acht Jahren teilnehmen, haben durchschnittlich fünf bis sechs Fortbildungen besucht (5.6) (Stiftung Haus der kleinen Forscher, 2017b).

\section{Wirkungen auf Personen- und Einrichtungsebene}

Die Wirkungen des Bildungsangebots der Initiative „Haus der kleinen Forscher“ werden als Veränderungen verstanden, die durch die Nutzung der Fortbildungsinhalte ausgelöst werden können. Sie zeigen sich auf verschiedenen Ebenen. Sowohl bezüglich des Lern- und Transfererfolgs auf Ebene der pädagogischen Fachund Lehrkräfte als auch auf Ebene der Einrichtungen gibt es bereits Erkenntnisse zur Wirkung der Fortbildungen des „Hauses der kleinen Forscher“. Anhand stiftungseigener Monitoring-Daten konnte gezeigt werden, dass die pädagogischen Fach-, Lehr- und Leitungskräfte mit dem Fortbildungsangebot sehr zufrieden sind (Stiftung Haus der kleinen Forscher, 2020). Im Zusammenhang mit der Evaluation der Angebote zur Bildung für nachhaltige Entwicklung wurde deutlich, dass die Kita-Leitungen als Multiplikatorinnen und Multiplikatoren in der Einrichtung agieren. Sie tragen das Bildungskonzept in die Einrichtung und teilen ihr Wissen mit dem Team (Stiftung Haus der kleinen Forscher, 2017b, 2020). Insgesamt legen die Ergebnisse nahe, dass Pädagoginnen und Pädagogen mit dem Besuch von „Haus der kleinen Forscher“-Fortbildungen ihr fachdidaktisches Wissen, ihre Motivation sowie ihre Selbstwirksamkeit in Bezug auf das Entdecken und Forschen mit Kindern verändern. Es gibt zudem Hinweise, dass sich der Besuch von „Haus der kleinen Forscher"-Fortbildungen auf die praktische Umsetzung des Gelernten in der Interaktion mit den Kindern auswirkt: Pädagogische Fach- und Lehrkräfte forschen anschließend häufiger mit den Kindern und lassen ihnen bei diesem Prozess mehr Freiräume (Stiftung Haus der kleinen Forscher, 2017b). 
Zwei externe Studien zur Arbeit der Stiftung „Haus der kleinen Forscher“ konnten ebenfalls Wirkungen des Fortbildungsangebots auf Ebene der Erwachsenen wir auch der Kinder zeigen. Das Forschungsprojekt „Early Steps into Science“ (Steffensky et al. 2018) stellte fest, dass die Teilnahme an naturwissenschaftlichen Fortbildungen in einem positiven Zusammenhang mit den naturwissenschaftlichen professionellen Kompetenzen pädagogischer Fachkräfte steht. Zudem zeigen Kinder aus Einrichtungen mit einem expliziten naturwissenschaftlichen Schwerpunkt höhere Lernfreude und höheres Selbstvertrauen in Bezug auf Naturwissenschaften als Kinder in Einrichtungen ohne naturwissenschaftlichen Schwerpunkt. Die Studie „Early Steps into Science and Literacy“ (Rank, Wildemann, Pauen, Hartinger, Tietze \& Kästner, 2018) gibt Hinweise darauf, dass naturwissenschaftliche Fortbildungen die naturwissenschaftlichen Kompetenzen wie auch den Spracherwerb der Kinder unterstützen und die Prozessqualität der Fachkräfte in naturwissenschaftsbezogenen Situationen bestimmen können.

Weitere Erkenntnisse zu Fortbildungswirkungen aus Längsschnitterhebungen der Stiftung werden im Beitrag B dieses Bandes berichtet. 


\section{Hintergründe der externen Studie und stiftungsinternen Befragung zur Professionalisierung von pädagogischen Fach- und Lehrkräften}

Die Stiftung „Haus der kleinen Forscher“ versteht sich als lernende Organisation, die ihre Angebote wissenschaftlich fundiert, sich von namhaften Expertinnen und Experten unterschiedlicher Fachrichtungen beraten lässt und durch die Förderung externer Begleitforschung vertiefende Erkenntnisse zu ihren Angeboten erhält sowie darauf aufbauend ihr pädagogisch-didaktisches Konzept kontinuierlich weiterentwickelt. Die unabhängige Begleitforschung sowie die stiftungseigenen Monitoring- und Evaluationsmaßnahmen bilden dabei ein Gesamtkonzept und generieren sich ergänzende Erkenntnisse zu den Wirkungen von Fortbildungen zur frühen MINT-Bildung für nachhaltige Entwicklung.

In früheren Aktivitäten zur Fachfundierung und Begleitforschung standen die Erarbeitung und Untersuchung von Kompetenzmodellen im Fokus. Derzeit und künftig sollen im Rahmen der Forschungsvorhaben der Stiftung „Haus der kleinen Forscher"verstärkt Entwicklungsmodelle erarbeitet und der Blick vermehrt auf die biografische Entwicklung auf Personen- wie auf Einrichtungsebene gerichtet werden. Ein solcher prozessorientierter Forschungsschwerpunkt knüpft an die bisherigen kompetenzorientierten Wirkungsstudien an.

\section{Fragestellung und Motivation der externen Studie und stiftungsinternen Längsschnittbefragung}

Für die bedarfsorientierte Angebotsentwicklung der Stiftung sind Erkenntnisse zu Merkmalen, die mit der MINT-bezogenen professionellen Entwicklung pädagogischer Fach- und Lehrkräfte zusammenhängen, von besonderem Interesse. Um diese Erkenntnisse zu gewinnen, hat die Stiftung zum einen gemeinsam mit dem Bundesministerium für Bildung und Forschung (BMBF) die externe Studie „Entwicklungsverläufe von pädagogischen Fach- und Lehrkräften in der frühen MINTBildung“ (kurz: „EpFL MINT“) gefördert. Diese wurde durchgeführt von einer Forschungsgruppe der Goethe-Universität Frankfurt, bestehend aus Nina Skorsetz, Lisa Öz, Julia Katharina Schmidt und Diemut Kucharz (Laufzeit 2017-2019). Zum anderen hat die Stiftung eine interne Längsschnittbefragung initiiert, um Informationen über Veränderungen zu erhalten, die durch die Teilnahme am Fortbildungsprogramm der Initiative „Haus der kleinen Forscher“ angestoßen werden. Auf den Erkenntnissen der externen Studie und der stiftungsinternen Befragungen auf- 
bauend können Erkenntnisse über die verschiedenen Lernbedarfe von Pädagoginnen und Pädagogen im frühen MINT-Bereich abgeleitet werden.

Mit der externen „EpFL MINT“-Studie (siehe Beitrag A) sollten erste Einblicke in die professionelle Entwicklung pädagogischer Fach- und Lehrkräfte für den Bildungsbereich MINT gewonnen werden. Es ist insgesamt noch recht wenig darüber bekannt, was pädagogische Fach- und Lehrkräfte motiviert, an MINT-bezogenen Fortbildungen teilzunehmen, welche Faktoren die Weiterqualifizierung im Bereich der frühen MINT-Bildung fördern oder verhindern und wie sich dies im Laufe ihres Professionalisierungsprozesses verändert (vgl. u. a. Friederich, 2019; Nationales MINT Forum, 2018). Die Forscherinnen untersuchten in einer explorativen Herangehensweise die „MINT-biografische“ Professionalisierung pädagogischer Fachund Lehrkräfte im Zusammenhang mit Fortbildungsbesuchen bei der Bildungsinitiative „Haus der kleinen Forscher“. Sie nahmen insbesondere die Fragen in den Blick, wie sich die selbstwahrgenommenen Entwicklungsverläufe der pädagogischen Fach- und Lehrkräfte in Bezug auf die Professionalisierung im MINT-Bereich beschreiben lassen und ob verschiedene Phasen oder prägende Ereignisse eine besondere Rolle in ihrer professionellen Entwicklung spielen. Dafür wurden sowohl Unterschiede zwischen erfahrenen und weniger erfahrenen Pädagoginnen und Pädagogen als auch zwischen pädagogischen Fach- und Grundschullehrkräften analysiert.

Die stiftungsinterne Längsschnittbefragung (siehe Beitrag B) ist als jährliche Erhebung unter den Pädagoginnen und Pädagogen geplant, die am Fortbildungsprogramm der Stiftung teilnehmen, um Wirkungen der Bildungsangebote der Stiftung zu früher MINT-Bildung auf die Kompetenzen der pädagogischen Fach-, Lehrkräfte und Leitungskräfte gezielt abbilden zu können. So kann die Stiftung die professionelle Entwicklung der Pädagoginnen und Pädagogen durch ihre Teilnahme am Fortbildungsangebot der Bildungsinitiative „Haus der kleinen Forscher“

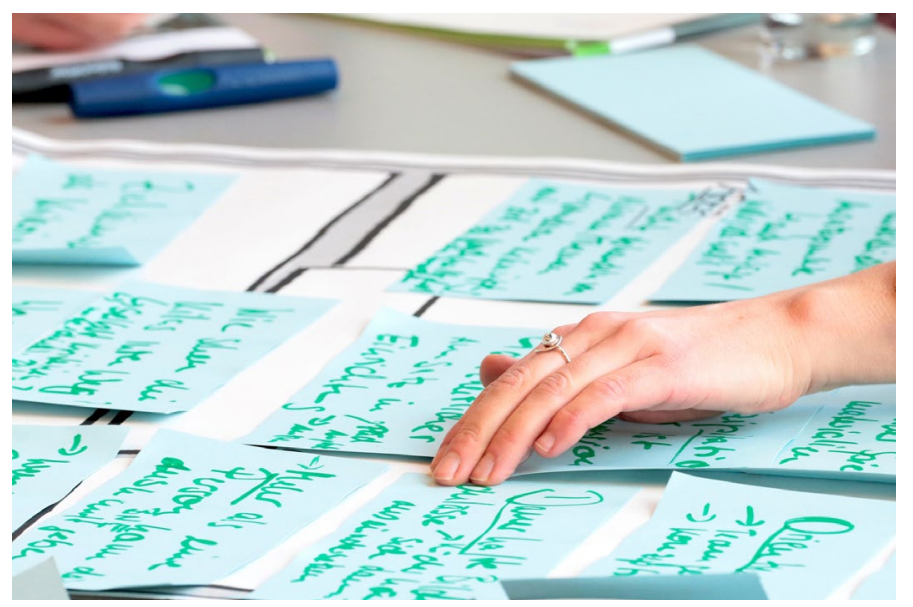

nachzeichnen.

In der bisher vorliegenden längsschnittlichen Evaluation der Fortbildungsangebote wird die professionelle Kompetenzentwicklung von Pädagoginnen und Pädagogen, die mind. eine Fortbildung der Bildungsinitiative „Haus der kleinen Forscher“ besucht haben, über drei Messzeitpunkte analysiert und der Zusammenhang von individuellen Eigenschaften und organisa- 
tionalen Rahmenbedingungen mit der Kompetenzentwicklung betrachtet. Dabei dient die Befragung vor dem Besuch einer „Haus der kleinen Forscher“-Fortbildung (erster Messzeitpunkt) der Erfassung einer Baseline: Welche MINT-bezogenen Kompetenzen bringen die Pädagoginnen und Pädagogen mit, die eine Fortbildung der Initiative „Haus der kleinen Forscher“ besuchen? Anhand eines Vergleichs dieser Daten mit den Ergebnissen einer Folgebefragung direkt im Anschluss an die besuchte Fortbildung (zweiter Messzeitpunkt) können unmittelbare Veränderungen der MINT-bezogenen Kompetenzen nach dem Besuch der Fortbildung in den Blick genommen werden. Der dritte Messzeitpunkt liefert wichtige Hinweise darauf, wie sich die Kompetenzentwicklung der Pädagoginnen und Pädagogen durch den Fortbildungsbesuch mittelfristig über einige Monate hinweg gestaltet.

\section{Qualitätssicherung, Unabhängigkeit und Wissenschaftliche Begleitung der Studien}

Der Wissenschaftliche Beirat der Stiftung „Haus der kleinen Forscher“ (Vorsitz: Prof. Dr. Hans-Günther Roßbach) berät diese zu Forschungsfragen sowie zur fachlichen Fundierung des Stiftungsangebots. ${ }^{4}$ Bei der konzeptuellen Vorbereitung und Ausschreibung der externen Studie zur Entwicklung von pädagogischen Fachund Lehrkräfte in der frühen MINT-Bildung hat der Wissenschaftliche Beirat beraten und die Durchführung des Vorhabens befürwortet. Die Studie wurde offen ausgeschrieben; das Verfahren wurde von der Stiftung koordiniert.

Eine unabhängige wissenschaftliche Jury unter Vorsitz von Prof. Dr. HansGünther Roßbach sichtete, unterstützt durch externe Fachgutachterinnen und -gutachter, die im Rahmen der öffentlichen Ausschreibung im Jahr 2017 eingegangenen Anträge. Die Jury bewertete die Anträge anhand wissenschaftlicher Qualitätskriterien und sprach sich für die Umsetzung der Studie „Entwicklungsverläufe von pädagogischen Fach- und Lehrkräften in der frühen MINT-Bildung“ („EpFL MINT“) durch die Forschungsgruppe der Goethe-Universität Frankfurt, bestehend aus Nina Skorsetz, Lisa Öz, Julia Katharina Schmidt und Diemut Kucharz, aus. Die Stiftung folgte dieser Förderempfehlung.

Von 2017 bis 2019 wurde die Studie „EpFL MINT“ von der Forschungsgruppe durchgeführt. Die Studie war als explorative Querschnittstudie angelegt, um retrospektiv die subjektiv wahrgenommenen Entwicklungsverläufe von pädagogischen Fach- und Lehrkräften zu erfassen, die Fortbildungsmaßnahmen im Rahmen des „Hauses der kleinen Forscher“ durchlaufen hatten. Dazu wurde eine

4 Die Protokolle der Beiratssitzungen sind auf der Webseite der Stiftung verfügbar (www.haus-der-kleinen-forscher.de), zu finden unter der Rubrik „Wissenschaftliche Begleitung“. 
ausgewählte Gruppe interviewt und eine weitere große Stichprobe standardisiert online befragt.

Die zentralen Fragestellungen und Ergebnisse sind im Abschnitt „Zusammenfassung zentraler Ergebnisse“ (siehe S. 38ff.) zu finden sowie vertiefend im Abschlussbericht der Studie (siehe Beitrag A).

Die Stiftung organisierte die administrative Betreuung des Studienprojekts. Beispielsweise unterstützte sie die Forschungsgruppe bei der Zusammenstellung der Stichprobe auf Basis bereits vorhandener Daten zu bisherigen Fortbildungsbesuchen von pädagogischen Fach- und Lehrkräften sowie bei der Kommunikation mit den lokalen Netzwerkpartnern und den Einrichtungen. Eine inhaltlichewissenschaftliche Beratung erfolgte durch den Wissenschaftlichen Beirat. Dieser befasste sich in seinen Sitzungen regelmäßig mit der Studie und beriet die Forschungsgruppe während der Durchführung.

Der Wissenschaftliche Beirat befürwortete auch die Durchführung der stiftungsinternen Längsschnittbefragung als eine Weiterentwicklung der stiftungseigenen Monitoring- und Evaluationsmaßnahmen. Diese baute auf bisherigen Erkenntnissen zu Wirkungen der Fortbildungen der Initiative „Haus der kleinen Forscher“ auf (Rank et al., 2018; Steffensky et al., 2018; Stiftung Haus der kleinen Forscher, 2020). Das Befragungsdesign sowie die ersten Ergebnisse wurden in zwei Sitzungen des Beirats (2018 und 2019) vorgestellt und diskutiert. Die Empfehlungen des Beirats wurden in der weiteren Erhebungs- und Analysearbeit der Längsschnittbefragung einbezogen. Beispielsweise hat die Stiftung auf Empfehlung des Beirats neben der Fortbildungsanzahl auch die Fortbildungsfrequenz (Anzahl besuchter Fortbildungen pro Jahr) in den Analysen berücksichtigt. In den kommenden Erhebungen des Längsschnitts rücken Häufigkeit und Qualität der Umsetzung der Fortbildungsinhalte (Ebene der Performanz) stärker in den Fokus. Dies wurde durch den Beirat empfohlen.

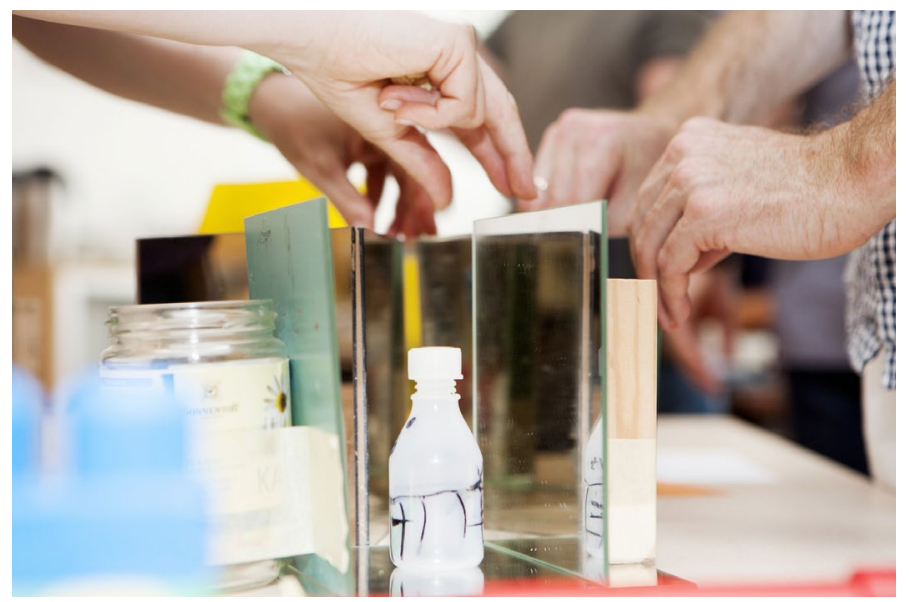

Im vorliegenden Band wird der Abschlussbericht der Studie „EpFL MINT“ zur Professionalisierung der pädagogischen Fachund Lehrkräfte veröffentlicht (siehe Beitrag A) und bisherige Ergebnisse der stiftungsinterne Längsschnittbefragung werden dargestellt (siehe Beitrag B). Die Studienberichte stellen neben Fragestellungen, Studiendesign und wesentlichen Ergebnissen 
auch Empfehlungen für die bedarfsgerechte und wirkungsorientierte Angebotsentwicklung in den Fokus und geben der Stiftung „Haus der kleinen Forscher“ Orientierung für die Qualitätsentwicklung ihrer Angebote. Die Umsetzung der wissenschaftlichen Empfehlungen in den inhaltlichen Angeboten der Stiftung „Haus der kleinen Forscher“ und ihrer fachlichen Weiterentwicklung werden im Schlusskapitel des Bandes beschrieben. 


\section{Zusammenfassung zentraler Ergebnisse}

Stiftung Haus der kleinen Forscher

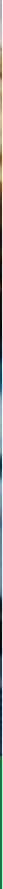




\section{Zusammenfassung zentraler Ergebnisse}

Der dreizehnte Band der Schriftenreihe „Wissenschaftliche Untersuchungen zur Arbeit der Stiftung ,Haus der kleinen Forscher“" stellt die professionelle Entwicklung von pädagogischen Fach- und Lehrkräften in den Fokus.

Um ihre Stiftungsangebote noch bedarfsorientierter und wirksamer (weiter-) zu entwickeln, hat die Stiftung „Haus der kleinen Forscher“, gemeinsam mit dem Bundesministerium für Bildung und Forschung, die Studie „Entwicklungsverläufe von pädagogischen Fach- und Lehrkräften in der frühen MINT-Bildung“(„EpFL MINT“) gefördert sowie im Rahmen ihrer stiftungsinternen Evaluationsmaßnahmen längsschnittliche Befragungen durchgeführt, die Erkenntnisse über die professionelle Entwicklung von pädagogischen Fach- und Lehrkräften im Bereich der frühen MINT-Bildung fokussieren.

Beide Untersuchungen haben pädagogische Fach- und Lehrkräfte, die mindestens eine Fortbildung der Initiative „Haus der kleinen Forscher“ besucht haben, zu ihrer Kompetenzentwicklung befragt. Das unterschiedliche methodische Design der beiden Erhebungen ermöglicht eine Untersuchung aus zwei analytischen Perspektiven. Aus den sich ergänzenden Ergebnissen lassen sich Erkenntnisse für weitere Erhebungen sowie für die inhaltlichen Stiftungsangebote ableiten (vgl. Diskussion).

\section{Entwicklungsverläufe von pädagogischen Fach- und Lehrkräften in der frühen MINT-Bildung (,EpFL MINT“)}

\section{Hintergrund und Fragestellung}

Im Projekt „Entwicklungsverläufe von pädagogischen Fach- und Lehrkräften in der frühen MINT-Bildung“ untersuchte die Forschungsgruppe bestehend aus Prof. Dr. Diemut Kucharz, Dr. Lisa Öz, Julia Katharina Schmidt und Dr. Nina Skorsetz (Goethe-Universität Frankfurt) die Entwicklung pädagogischer Fach- und Lehrkräfte, die an Bildungsangeboten der Initiative „Haus der kleinen Forscher“ teilgenommen haben, in ihrem selbst berichteten MINT-bezogenen Professionalisierungsprozess. Ziel der Studie war zu untersuchen, wie sich selbstwahrgenommene Entwicklungen pädagogischer Fach- und Lehrkräfte in Bezug auf die Professionalisierung im MINT-Bereich beschreiben lassen und ob verschiedene Phasen oder prägende Ereignisse eine besondere Rolle in der professionellen Entwicklung der pädagogischen Fach- und Lehrkräfte spielen.

Die Studie wurde von der Stiftung „Haus der kleinen Forscher“ und dem Bundesministerium für Bildung und Forschung (BMBF) gefördert. Folgende Fragen standen im Zentrum der Studie: 
1. Wie lassen sich die selbstwahrgenommenen Entwicklungsverläufe von pädagogischen Fach- und Lehrkräften in Bezug auf die Professionalisierung im MINT-Bereich beschreiben?

2. Gibt es verschiedene Phasen, Ereignisse oder typische Abläufe, die Fach- und Lehrkräfte für ihren Professionalisierungsprozess nennen?

Um diesen Fragen näherzukommen wurden folgende Teilfragestellungen in den Blick genommen:

1. Wo stehen pädagogische Fach- und Lehrkräfte in Bezug auf forschendes Lernen mit Kindern nach ihrer eigenen Einschätzung?

2. Gibt es Unterschiede zwischen erfahrenen und weniger erfahrenen pädagogischen Fach- und Lehrkräften?

3. Gibt es Unterschiede zwischen pädagogischen Fachkräften und Grundschullehrkräften?

4. Wie lassen sich die Qualifizierungsbedarfe beschreiben?

\section{Durchführung}

Die explorative Querschnittstudie nutzte ein sequenzielles Mixed-Methods-Design, bei dem aufbauend auf 15 qualitativen Interviews mit pädagogischen Fachund Lehrkräften ein Fragebogen entwickelt wurde. Die Stichprobe der darauf folgenden quantitativen Online-Befragung umfasste knapp 400 pädagogische Fach- und Lehrkräfte, die an mindestens einer Fortbildung der Initiative „Haus der kleinen Forscher" teilgenommen hatten. Die Ergebnisse basieren damit auf Selbsteinschätzungen der teilnehmenden Fach- und Lehrkräfte.

Die teilnehmenden pädagogischen Fach- und Lehrkräfte wurden nach ihrer Einschätzung bezüglich der drei MINT-bildungsbezogenen professionellen Kompetenzen Professionswissen, Motivation und Einstellungen gefragt. Die Kompetenzentwicklung wurde zum einen über den Vergleich der Aussagen von erfahrenen und weniger erfahrenen pädagogischen Fach- und Lehrkräften nachgezeichnet. Die Erfahrung wurde dafür hinsichtlich der Berufserfahrung ${ }^{5}$, der Anzahl der besuchten Fortbildungen ${ }^{6}$ und einem möglichen MINT-Hintergrund ${ }^{7}$

5 Gruppenvergleich mit den drei Gruppen Berufsanfängerinnen und -anfänger (o bis 2 Jahre), Fortgeschrittene ( 3 bis 10 Jahre) und Profis (mehr als 10 Jahre).

6 Gruppenvergleich mit den vier Gruppen 1 bis 2, 3 bis 6, 7 bis 9 und mehr als 9 besuchte Fortbildungen.

7 Von einem MINT-Hintergrund wurde gesprochen, wenn ein MINT-Fach Teil der Ausbildung der Befragten war oder diese unabhängig von den Stiftungsangeboten bereits eine Weiterbildung im MINTBereich besucht hatten. 
unterschieden. Zum anderen schätzten die Fach- und Lehrkräfte die Entwicklung ihres Professionswissens durch eine retrospektive Befragung ein.

\section{Zentrale Ergebnisse der Studie}

1. Die pädagogischen Fach- und Lehrkräfte schätzen ihre Motivation, ihr Wissen und ihre Einstellungen zur frühen MINT-Bildung positiv ein.

Eine deutliche Mehrheit der pädagogischen Fach- und Lehrkräfte bewertet die frühe MINT-Bildung sowohl in Kitas als auch in Grundschulen als wichtig. Die Befragten schätzen ihr Wissen zum Entdecken und Forschen mit Kindern hoch ein. Zudem trauen sie sich in hohem Maße zu, Kinder beim entdeckenden und forschenden Lernen zu begleiten.

\section{Die Anzahl der besuchten Fortbildungen der Initiative „Haus der kleinen} Forscher" ist von zentraler Bedeutung für die professionelle Entwicklung in der frühen MINT-Bildung.

Professionswissen: Um den Entwicklungsprozess im Professionswissen abzubilden, scheint das Merkmal „Anzahl der besuchten Fortbildungen“ relevant. Die Befragten geben rückblickend an, dass mit zunehmendem Fortbildungsbesuch ihr selbstwahrgenommenes Professionswissen steigt - die Fortbildungen der Initiative „Haus der kleinen Forscher“ werden dabei als zentrale Lerngelegenheiten beschrieben. Die Berufserfahrung in Jahren und der MINT-Hintergrund der befragten Fach- und Lehrkräfte konnten hingegen berichtete Kompetenzzuwächse nicht erklären.

Motivation: Pädagogische Fach- und Lehrkräfte mit MINT-Hintergrund und längerer Berufserfahrung sind bezüglich der Beschäftigung mit MINT-Themen - beruflich als auch privat - motivierter als Pädagoginnen und Pädagogen ohne MINTHintergrund beziehungsweise mit weniger Berufserfahrung. Die Anzahl besuchter Fortbildungen trägt signifikant zu einer Steigerung der MINT-spezifischen Motivation bei. Eine Stagnation dieser positiven Motivationsentwicklung tritt bei dem Besuch von neun und mehr Fortbildungen ein.

Einstellungen: Im Bereich der Einstellungen zur Bedeutung einer frühen MINT-Bildung und dem Forschen mit Kindern lassen sich geringe positive Entwicklungen aus den Antworten der Befragten identifizieren. 
3. Lehrkräfte zeigen eine höhere Selbstwirksamkeitserwartung als pädagogische Fachkräfte. Es deutet sich jedoch an, dass pädagogische Fachkräfte in Hinblick auf fachdidaktisches Wissen stärker von dem Besuch der Fortbildungen profitieren.

Lehrkräfte weisen hinsichtlich der Motivation zum Forschen eine höhere Selbstwirksamkeitserwartung auf, wohingegen die Fachkräfte häufiger an Fortbildungen teilnahmen und Beispielversuche im pädagogischen Alltag durchführen. In der retrospektiven Einschätzung des fachdidaktischen Wissens zeigt sich, dass die Lehrkräfte direkt nach der Ausbildung ihr fachdidaktisches Wissen höher einschätzen als die pädagogischen Fachkräfte. Die pädagogischen Fachkräfte schätzen wiederum ihr fachdidaktisches Wissen nach der (von den Fachkräften vor der Befragung zuletzt besuchten) Fortbildung höher ein, als dies Lehrkräfte tun.

\section{Die Fortbildungen unterstützen die Pädagoginnen und Pädagogen dabei, Hemmnisse gegenüber dem Forschen zu MINT-Themen abzubauen.}

Als fördernde Merkmale für ihre Professionalisierung durch Fortbildungen nannten die Befragten das eigene Entdecken und Forschen, die Verknüpfung von Theorie und Praxis in der Fortbildung sowie das regelmäßige und vielfältige Fortbildungsangebot der Initiative. Fortbildungen dienten auch dazu, Hemmnisse und Ängste hinsichtlich des Forschens im MINT-Bereich abzubauen und die Selbstwirksamkeitserwartung zu steigern, insbesondere bei pädagogischen Fachkräften.

\section{Längsschnittbefragung zu Wirkungen des „Haus der kleinen Forscher"-Fortbildungsangebots}

\section{Hintergrund und Fragestellung}

Die in diesem Band vorgestellten Analysen der stiftungsinternen Längsschnittbefragung gehen der Frage nach, welche durchschnittliche kurz- und mittelfristige Kompetenzentwicklung Pädagoginnen und Pädagogen nach dem Besuch einer „Haus der kleinen Forscher“-Präsenzfortbildung zeigen, welche individuellen Eigenschaften und organisationalen Rahmenbedingungen in Zusammenhang mit der Effektivität der Fortbildungsteilnahme stehen und wie das Ausgangsniveau verschiedener Kompetenzen mit der Anzahl bereits besuchter Fortbildungen und der Fortbildungsfrequenz (Anzahl bereits besuchter Fortbildungen pro Jahr) zusammenhängt. Folgende Fragestellungen standen entsprechend im Fokus der Analysen: 
1. Welche durchschnittliche kurz- und mittelfristige Kompetenzentwicklung zeigen Pädagoginnen und Pädagogen nach dem Besuch einer „Haus der kleinen Forscher"-Präsenzfortbildung?

2. Wie unterscheiden sich die kurz- und mittelfristigen Wirkungen der Teilnahme an einer Präsenzfortbildung für Personen mit unterschiedlichen individuellen Eigenschaften und organisationalen Rahmenbedingungen?

3. Welche Zusammenhänge zeigen sich zwischen der Fortbildungsanzahl und -frequenz vor dem betrachteten Fortbildungsbesuch und dem Ausgangsniveau verschiedener Kompetenzen?

\section{Durchführung}

Zur Beantwortung der aufgeführten Forschungsfragen kam in der vorliegenden Studie ein Längsschnittdesign mit drei Messzeitpunkten zur Anwendung. An den ersten beiden Messzeitpunkten der Längsschnittbefragung nahmen zwischen September 2018 und Februar 2019 deutschlandweit 1.559 Pädagoginnen und Pädagogen teil. In 167 „Haus der kleinen Forscher“-Fortbildungen wurden die Teilnehmenden vor sowie direkt nach einer Fortbildung befragt. An einer Folgebefragung einige Monate nach dem Fortbildungsbesuch nahmen 453 der befragten Pädagoginnen und Pädagogen erneut teil.

Die Effekte des Fortbildungsbesuchs auf die Kompetenzentwicklung der befragten Pädagoginnen und Pädagogen wurden mithilfe von Varianz- und Regressionsanalysen untersucht. In der Analyse wurden ausschließlich Daten von Personen berücksichtigt, die zu allen drei Messzeitpunkten an der Befragung teilgenommen haben. Die angewendeten Verfahren ermöglichten es, Effekte des Fortbildungsbesuchs unter Berücksichtigung individueller Eigenschaften und organisationaler Rahmenbedingungen zu identifizieren.

Auf diese Weise wurde der Zusammenhang von individuellen Eigenschaften und organisationalen Rahmenbedingungen auf die Kompetenzentwicklung untersucht, um sich der Frage zu nähern, ob ein Fortbildungsbesuch für Personen mit bestimmten Merkmalen besonders wirksam ist. 


\section{Zentrale Ergebnisse der Längsschnittbefragung}

\section{Die Pädagoginnen und Pädagogen schätzen ihr fachdidaktisches Wissen, ihre Motivation und Selbstwirksamkeit nach einem Fortbildungsbesuch signifikant höher ein.}

Die befragten Pädagoginnen und Pädagogen zeigen nach dem Besuch einer „Haus der kleinen Forscher“-Präsenzfortbildung durchschnittlich sowohl kurz-als auch mittelfristige Teilnahmeeffekte auf die Kompetenzentwicklung. Die Pädagoginnen und Pädagogen schätzen ihr fachdidaktisches Wissen nach dem Besuch einer „Haus der kleinen Forscher“-Fortbildung bedeutsam höher ein, sie empfinden sich als leicht motivierter, mit Kindern zu entdecken und zu forschen, und sie trauen sich dies auch eher zu. Die Ergebnisse weisen darauf hin, dass der Besuch einer Fortbildung insgesamt einen stärkeren Zusammenhang mit dem fachdidaktischen Wissen hat als mit den Kompetenzen Motivation und Selbstwirksamkeit.

\section{Die Effekte einer Fortbildungsteilnahme hängen mit individuellen Eigenschaften der Pädagoginnen und Pädagogen sowie mit organisationalen Rahmenbedingungen zusammen.}

Pädagoginnen und Pädagogen mit geringem Ausgangsniveau in ihren Kompetenzen und wenig Berufserfahrung scheinen stärker von der Teilnahme an einer einzelnen Präsenzfortbildung zu profitieren. Es konnten keine Hinweise auf praktisch bedeutsame Unterschiede in der selbsteingeschätzten Kompetenzentwicklung zwischen pädagogischen Fach-, Lehr- und Leitungskräften gefunden werden, alle pädagogischen Zielgruppen profitieren gleichermaßen vom Besuch einer „Haus der kleinen Forscher"-Fortbildung. Auf Ebene der organisationalen Rahmenbedingungen scheint insbesondere der MINT-bezogene Betreuungsschlüssel eine Rolle zu spielen. Er stellt das Verhältnis der Anzahl aller Pädagoginnen und Pädagogen, die zu MINT-Bildungsinhalten entdecken und forschen, zur Anzahl der Kinder in der Einrichtung dar. Dieser hängt positiv mit der Entwicklung des fachdidaktischen Wissens, der Motivation und Selbstwirksamkeit zusammen.

\section{Je mehr Fortbildungen Pädagoginnen und Pädagogen besucht haben, umso} ausgeprägter scheint ihre Kompetenzentwicklung.

Die Anzahl zuvor besuchter „Haus der kleinen Forscher“-Fortbildungen hängt mit der Entwicklung der Kompetenzen fachdidaktisches Wissen, Motivation sowie Selbstwirksamkeit in Bezug auf das Entdecken und Forschen mit Kindern zusammen. Pädagoginnen und Pädagogen, die zuvor mehr Fortbildungen besucht haben, weisen ein höheres selbsteingeschätztes Ausgangsniveau auf - sowohl für das fachdidaktische Wissen als auch für die Motivation und Selbstwirksamkeit, 
mit Kindern zu entdecken und zu forschen. Diese Ergebnisse sind Hinweise auf längerfristige Effekte der Fortbildungen.

Die Ergebnisse der „EpFL MINT“-Studie sowie der stiftungsinternen Längsschnittbefragung weisen in eine ähnliche Richtung. Beide Untersuchungen weisen auf einen Zusammenhang zwischen der Anzahl besuchter „Haus der kleinen Forscher"-Präsenzfortbildungen und der Kompetenzentwicklung der teilnehmenden Pädagoginnen und Pädagogen hin. Dieser deutet sich in beiden Untersuchungen in Hinblick auf die Entwicklung des fachdidaktisches Wissen und der Motivation an. Des Weiteren legen beide Untersuchungen nahe, dass weniger erfahrene Personen stärker vom Besuch einer „Haus der kleinen Forscher“-Fortbildung profitieren als Personen mit mehr Erfahrung. 


\section{A Entwicklungsverläufe von pädagogischen Fach- und Lehrkräften in der frühen MINT-Bildung}

Nina Skorsetz, Lisa Öz, Julia Katharina Schmidt, Diemut Kucharz

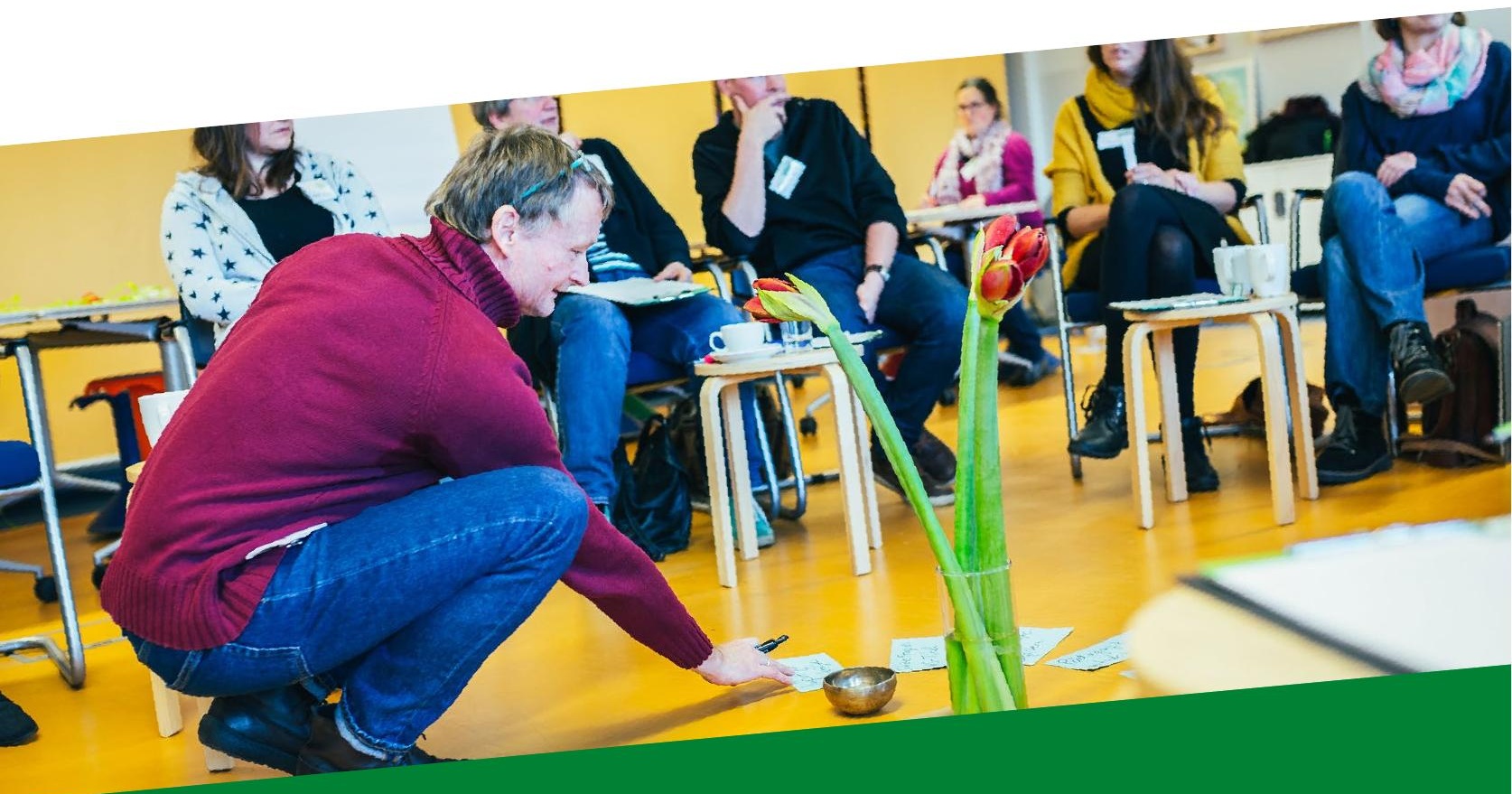

$1 \quad$ Einleitung

2 Theoretischer Hintergrund

3 Stand der Forschung

4 Forschungsfragen

5 Forschungsdesign und Stichprobenziehung

6 Instrumentenentwicklung und Durchführung

7 Auswertung

8 Ergebnisse der Studie

9 Diskussion der Ergebnisse

10 Grenzen der Studie

11 Schlussfolgerungen für die Arbeit der Stiftung „Haus der kleinen Forscher“ 


\section{$1 \quad$ Einleitung}

Bildung in der frühen Kindheit trägt maßgeblich dazu bei, zukünftige Entwicklungs-, Teilhabe- und Aufstiegschancen der heranwachsenden Kinder zu verbessern (Bundesministerium für Bildung und Forschung (BMBF), o. D.). Die aktuelle Bildungsforschung stellt insbesondere den Bereich der MINT-Fächer, also Mathematik, Informatik, Naturwissenschaften und Technik, als grundlegend für späteres erfolgreiches Lernen heraus, nicht zuletzt deshalb, weil unsere Lebenswelt zunehmend auf technischen Errungenschaften basiert. Es komme vor allem darauf an, dass Kinder schon in den ersten Lebensjahren mit diesen Thematiken in Berührung gebracht werden, um so ihre individuellen Fähigkeiten und Interessen optimal entwickeln zu können (Gold \& Dubowy, 2013). Um die ohnehin ausgeprägte kindliche Neugier in allen Belangen der sie umgebenden Natur zu stillen, weisen daher vermehrt Bildungsforscherinnen und -forscher aller Bereiche darauf hin, dass es wichtig ist, mit der MINT-Frühförderung der Jungen und Mädchen bereits in den Kindertagesstätten zu beginnen (Morgan, Farkas, Hillemeier \& Maczuga, 2016).

Die Stiftung „Haus der kleinen Forscher“ setzt an diesem Punkt an. In einer bundesweiten Initiative engagiert sie sich seit 2006 dafür, die Bildungschancen von Kindern im Kita- und Grundschulalter in den Bereichen Mathematik, Informatik, Naturwissenschaften und Technik zu verbessern. Zu diesem Zweck wurde ein Fortbildungsprogramm entwickelt, das pädagogische Fach- und Lehrkräfte darin unterstützt, MINT-spezifische Inhalte und Fragestellungen mit Kindern im Alter zwischen drei und zehn Jahren gemeinsam zu erforschen. Die Stiftung möchte damit einen Beitrag zur Qualifizierung des frühpädagogischen Personals und zur Qualitätsentwicklung von Einrichtungen sowie zur Persönlichkeits- und Interessenentwicklung der Kinder und zur Nachwuchsförderung in den MINT-Bildungsbereichen leisten (vgl. Stiftung Haus der kleinen Forscher, 2013, 2015b, 2019).

Im Projekt „Entwicklungsverläufe von pädagogischen Fach- und Lehrkräften in der MINT-Bildung“ („EpFL MINT“) untersuchte die Forschungsgruppe (Diemut Kucharz, Lisa Öz, Julia Katharina Schmidt und Nina Skorsetz) der Goethe-Universität Frankfurt, welche Entwicklungsverläufe die pädagogischen Fach- und Lehrkräfte in ihrer MINT-bezogenen Professionalisierung beschreiben, die an mindestens einer Fortbildung der Initiative „Haus der kleinen Forscher“ teilgenommen haben. Insbesondere sollten individuelle Lernbedarfe in verschiedenen Phasen der Entwicklung als pädagogische Fach- beziehungsweise Lehrkraft und die Umsetzung von Lerninhalten aus den besuchten Fortbildungen im pädagogischen Alltag der Kitas und Grundschulen in den Blick genommen werden. Gefördert wurde die Stu- 
die durch die Stiftung „Haus der kleinen Forscher“ gemeinsam mit dem Bundesministerium für Bildung und Forschung.

Der vorliegende Bericht stellt zunächst den theoretischen Hintergrund zu zentralen Teilbereichen der professionellen Kompetenz von pädagogischen Fachund Lehrkräften dar. Es folgt der aktuelle Stand der Forschung zur Bedeutung von Fortbildungen für die Entwicklung der professionellen Kompetenz sowie zu Professionswissen, Motivation und Einstellungen als zentrale Facetten der Professionalisierung pädagogischer Fach- und Lehrkräfte. Anschließend werden die hier untersuchten Forschungsfragen dargestellt sowie die Planung und Durchführung des Projekts beschrieben. Da es für die Betrachtung von Entwicklungsverläufen notwendig war, eigene empirische Messinstrumente zu entwickeln, liegt ein zentraler Aspekt dieses Berichts auf der Vorstellung dieser Instrumente sowie auf den Ergebnissen, Interpretationen und Grenzen der Studie. Der Bericht endet mit Schlussfolgerungen zur Entwicklung der drei Kompetenzfacetten Professionswissen, Motivation und Einstellungen. Zusätzlich werden abschließend Empfehlungen für die Arbeit der Stiftung „Haus der kleinen Forscher“ abgeleitet. 


\section{Theoretischer Hintergrund}

In diesem Projekt stehen die Entwicklungsverläufe von pädagogischen Fachund Lehrkräften im Fokus, die sich im Rahmen der Initiative „Haus der kleinen Forscher" fortgebildet haben. Entwicklungsverläufe werden in der vorliegenden Studie verstanden als Veränderungen im Prozess der Professionalisierung, wie sie von den Teilnehmerinnen und Teilnehmern selbst wahrgenommen werden. In Hinblick auf die Professionalisierung im MINT-Bereich stellen wir vor allem drei Teilbereiche professioneller Kompetenz als zentral heraus: Professionswissen, Motivation und Einstellungen (vgl. Stiftung Haus der kleinen Forscher, 2019). Diese drei Teilbereiche sollen im nächsten Schritt theoretisch untermauert werden.

\subsection{Professionelle Kompetenzen}

Um Entwicklungsverläufe in der Professionalisierung pädagogischer Fach- und Lehrkräfte untersuchen zu können, ist es von Relevanz, professionelle Kompetenz zu definieren und zu verstehen, was dieses Konstrukt umfasst. Wenn wir von Entwicklungsverläufen professioneller Kompetenz sprechen, gehen wir implizit davon aus, dass diese erlern- und veränderbar ist. In diesem Sinne stellt das „Modell der Determinanten und Konsequenzen der professionellen Kompetenz von Lehrkräften“ von Kunter et al. (2011) die professionelle Kompetenz von Fach- und Lehrkräften in ein Wirkungsgefüge. Hier wird die professionelle Kompetenz von Fach- und Lehrkräften als ein Zusammenspiel zwischen (1.) Lerngelegenheiten und deren Nutzung, (2.) persönlichen Voraussetzungen und (3.) professionellem Verhalten beschrieben. Die betreffenden Teilaspekte Nutzung von Lerngelegenheiten, professionelle Kompetenz und Verhalten sowie die persönlichen Voraussetzungen sind in Abbildung 3 dargestellt. Sie werden für die vorliegende Studie MINT-bezogen adaptiert und operationalisiert.

Brunner et al. (2006) beschreiben in Bezug zur Arbeit an der COAKTIV-Studie die professionelle Kompetenz als „das dynamische Zusammenwirken von Aspekten des Professionswissen, Überzeugungen, motivationalen Orientierungen und selbstregulativen Fähigkeiten“ (S. 523).

Lerngelegenheiten und deren Nutzung sind maßgeblich für die Verbesserung der professionellen Kompetenz. Die Fortbildungen zu MINT, die von der Initiative „Haus der kleinen Forscher“ angeboten werden, sind als solche Lerngelegenheiten zu begreifen. Sie sind den explizit geschaffenen Lerngelegenheiten zuzuordnen, welche als grundlegend für Kompetenzentwicklungen der pädagogischen Fach- und Lehrkräfte nach der Ausbildung angesehen werden (Kunter et al., 2011). Informelle Lerngelegenheiten finden sich im Alltag von pädagogischen 
Fach- und Lehrkräften sowohl bei der Arbeit in der Einrichtung mit den Kindern als auch beim Austausch mit Kolleginnen und Kollegen. Letzteres ist zudem im Rahmen von Fortbildungen präsent.

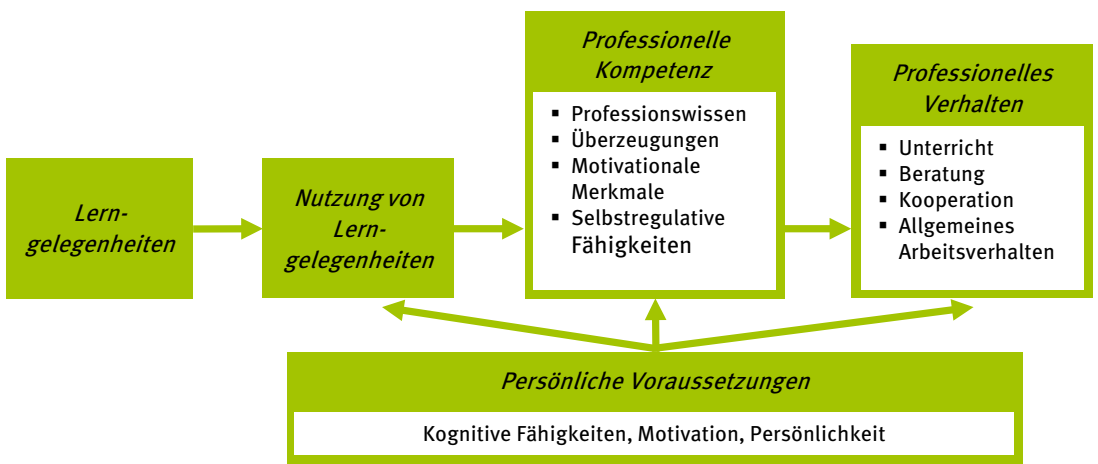

Abbildung 3. Ausschnitt aus „Modell der Determinanten und Konsequenzen der professionellen Kompetenz von Lehrkräften “ nach Kunter et al. (2011, S. 59)

\subsection{Professionswissen}

Professionswissen lässt sich nach Kunter et al. (2011) weiter in Fachwissen, fachdidaktisches Wissen, pädagogisch-psychologisches Wissen sowie Organisationsund Beratungswissen untergliedern.

Ein Überblick über die Spezifikationen des Professionswissens ist der folgenden Abbildung 4 zu entnehmen:

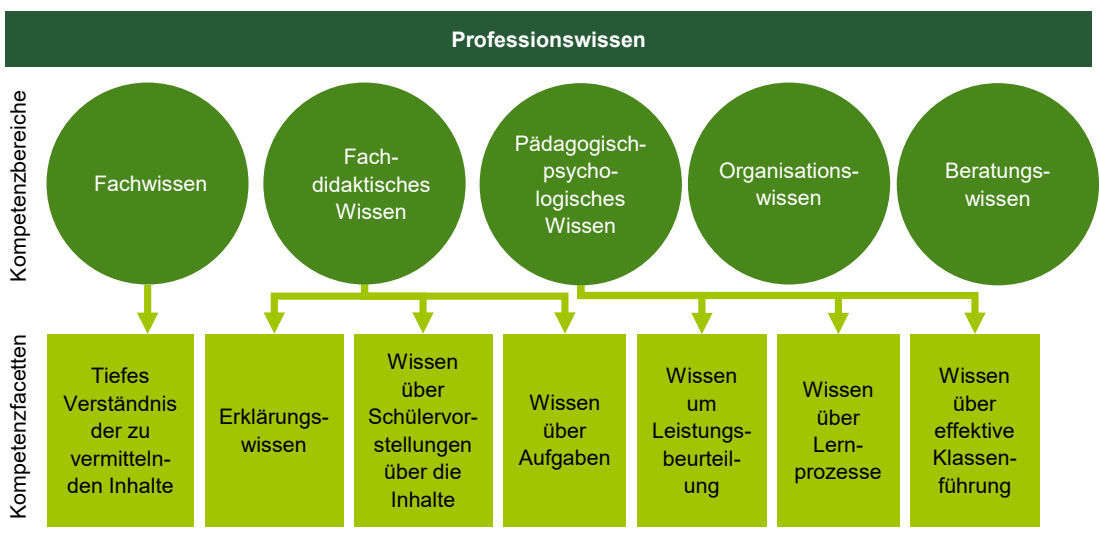

Abbildung 4. Spezifikationen für das Professionswissen, orientiert an Kunter et al. (2011, S. 32) 
Unter Fachwissen ist nach Brunner et al. (2006) nicht nur die fachliche Expertise, sondern zudem ein vertieftes Hintergrundwissen über die zu vermittelnden Inhalte hinaus zu verstehen, beispielsweise die Inhaltsstruktur oder der syntaktische Aufbau von Wissen in einem speziellen Fach. Es wird somit als Grundlage angesehen, um Lernprozesse zu steuern.

Fachdidaktisches Wissen bezeichnet die Verbindung zwischen Fachwissen und der Schülervorstellung zu diesen Inhalten beziehungsweise das Wissen darüber, wie Fachinhalte den Lernenden zugänglich gemacht werden können. Nach Neuweg (2011) ist dem fachdidaktischen Wissen auch das curriculare Wissen zuzuordnen. Darunter versteht er die Kenntnis darüber, welches Wissen vermittelt werden soll und welche Materialien und Medien sich situationsspezifisch eignen.

Im Rahmen der Arbeit der Stiftung „Haus der kleinen Forscher“ ist im Sinne des MINT-spezifischen fachdidaktischen Wissens der „Forschungskreis“ zu nennen, der als Werkzeug für Pädagoginnen und Pädagogen zum Entdecken und Forschen mit Kindern gesehen werden kann. Das didaktische Konzept einer naturwissenschaftlichen Grundbildung zum Forschen mit Kindern und das damit verbundene Modell des Forschungskreislaufs wurde von Frau Marquardt-Mau entwickelt (2004) und im pädagogischen Ansatz der Stiftung „Haus der kleinen Forscher“ adaptiert. ${ }^{8}$ „Der Prozess des Forschens gliedert sich [nach dem Forschungskreis] in verschiedene Phasen des Denkens und Handelns, die typischerweise in einem wiederkehrenden Zyklus auftreten:

(1) Frage an die Natur stellen,

(2) Ideen \& Vermutungen sammeln,

(3) Ausprobieren \& Versuch durchfuhren,

(4) Beobachten \& Beschreiben,

(5) Ergebnisse dokumentieren,

(6) Ergebnisse erörtern.“

(Marquardt-Mau, 2004, 2011; Rank, Wildemann, Hartinger \& Tietze, 2018; Stiftung Haus der kleinen Forscher, 2013). 


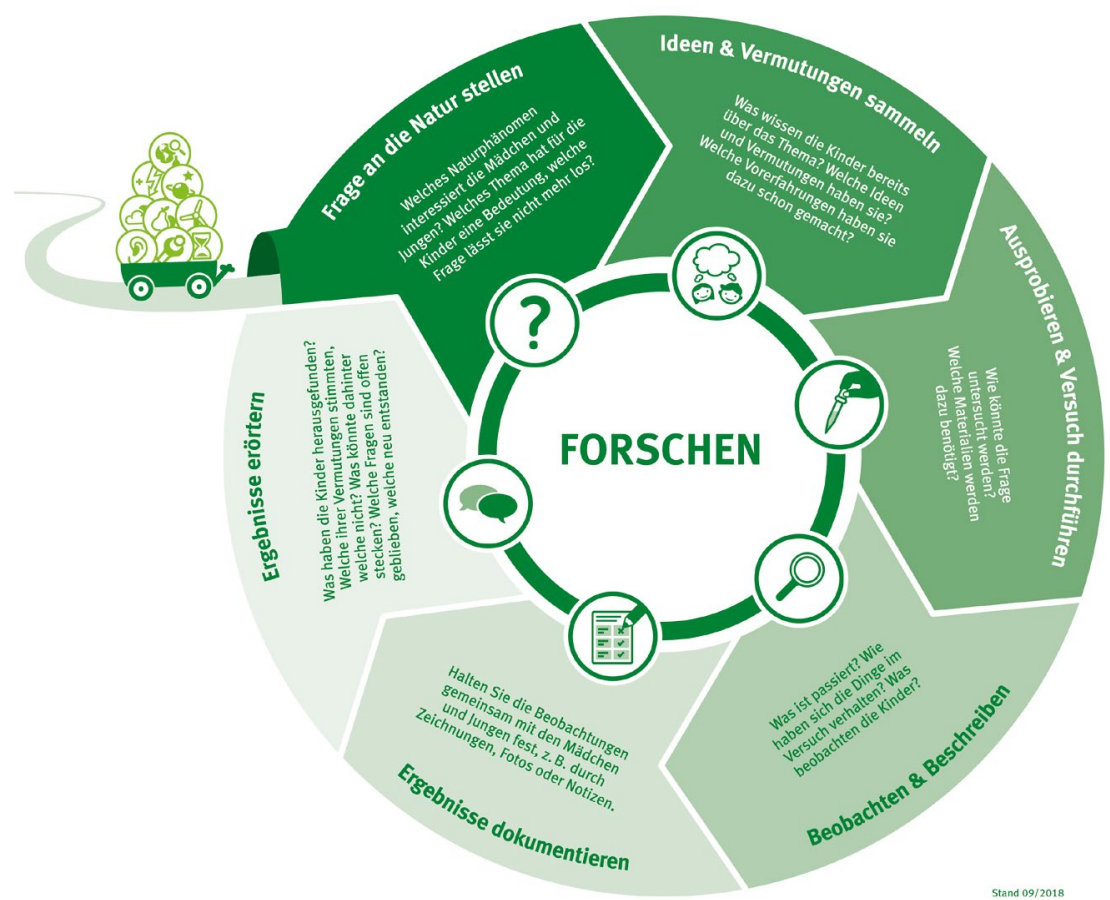

Abbildung 5. Die Methode „Forschungskreis“(in Anlehnung an Marquardt-Mau, 2011)

Der „Forschungskreis“ soll mit der Beschreibung des naturwissenschaftlichen Vorgehens als Prozess der Erkenntnisgewinnung Orientierung bieten, das Forschen mit Kindern ausgangsoffen und interaktiv zu gestalten (vgl. MarquardtMau, 2004, 2011; Rank et al., 2018; Stiftung Haus der kleinen Forscher, 2013).

Das pädagogisch-psychologische Wissen ist fachunabhängig und meint insbesondere Wissen über effektive Klassenführung sowie die Schaffung von adaptiven und kognitiv anregenden Lerngelegenheiten. Dazu braucht es spezifisches Wissen über Sozialformen, Methodik und die Organisation von Lernprozessen unter Berücksichtigung der Heterogenität von Lerngruppen, was Wissen über Entwicklungspsychologie und Lerntheorien sowie pädagogische Diagnostik beinhaltet. Beratungs- und Organisationswissen sind ebenfalls fachunspezifisch und beziehen sich auf die jeweilige Bildungseinrichtung.

Professionelles Verhalten zeigt sich vor allem an der Qualität der Lerngelegenheiten, die die pädagogische Fach- beziehungsweise Lehrkraft erzeugen kann. Das Potenzial für effektives professionelles Verhalten wird in erster Linie durch die professionelle Kompetenz der Fach- beziehungsweise Lehrkraft bestimmt (Kunter et al., 2011), hängt aber auch von den persönlichen Voraussetzungen, also den 
kognitiven Fähigkeiten, der Motivation sowie der Persönlichkeit ab. Diese persönlichen Voraussetzungen der pädagogischen Fach- beziehungsweise Lehrkraft fließen auch in die anderen beiden Aspekte Nutzung von Lerngelegenheiten und professionelle Kompetenz ein.

Im Rahmen dieser Studie kann das professionelle Verhalten nur durch die Beschreibung von Handlungs- beziehungsweise Verhaltensoptionen durch die Fachund Lehrkräfte selbst erfasst werden, die vom tatsächlichen Handeln im Alltag abweichen können. Aus dem Grund sind diese Aussagen, die eher einen Hinweis auf die Selbstwahrnehmung und -reflexion geben, dem fachdidaktischen und pädagogischen Wissen und damit dem Professionswissen zuzuordnen.

\subsection{Einstellungen und Überzeugungen}

„Einstellungen sind Gefühle, die auf unseren Überzeugungen beruhen und uns dazu prädisponieren, gegenüber Dingen, Menschen und Ereignissen in einer bestimmten Weise zu reagieren“ (Myers, 2014, S. 598). Beim Teilaspekt der Überzeugungen geht es, im Gegensatz zum pädagogisch-psychologischen Wissen, um den individuellen Richtigkeitsglauben (,beliefs“). Darunter werden nach Kunter et al. (2011) verschiedene Teilaspekte zusammengefasst: Wertbindungen, epistemologische Überzeugungen, subjektive Theorien über Lehren und Lernen sowie Zielsysteme.

Wertbindungen zielen auf die Einstellungen zur Tätigkeit als pädagogische Fach- und Lehrkraft und zur Bedeutung von MINT-Themen für die frühkindliche Bildung ab.

Epistemologische Überzeugungen umfassen persönliche Auffassungen über Wissen und dessen Entstehung. Überzeugungen über die Natur des Wissens betreffen Struktur (von Isolation bis Vernetzung) und Verlässlichkeit von Wissen (Veränderungen von Wahrheit in Raum und Zeit). Bei der Entstehung von Wissen geht es beispielsweise darum, ob „Wissensgenese als Übernahme von Wahrheiten [... oder ...] als soziale Konstruktionsleistung“ (Kunter et al., 2011, S. 236) aufgefasst wird oder die Koexistenz nebeneinanderstehender Theorien bewusst nachvollzogen wird.

Die subjektive Theorie über Lehren und Lernen einer pädagogischen Fachbeziehungsweise Lehrkraft beschreibt ihre persönliche Vorstellung davon, wie Lernende die zu vermittelnden Inhalte verarbeiten und wie sie aufgrund dessen unterrichtet werden sollten.

Zielsysteme bezeichnen Zielvorstellungen des Unterrichts beziehungsweise der Lerngelegenheiten. Überzeugungen beeinflussen maßgeblich das Verhalten gegenüber Kolleginnen und Kollegen, die Unterrichtsplanung und den Umstand, inwiefern Neues übernommen wird (Kunina-Habenicht, Decker \& Kunter, 2016). 
Besonders bedeutsam bezüglich der Einstellungen der pädagogischen Fachund Lehrkräfte werden ihre Überzeugungen und Werthaltungen dahingehend sein, welche Bedeutung sie der frühen MINT-Förderung in Kitas und Grundschulen beimessen und ob die Beschäftigung mit MINT-Themen ihnen selbst Freude bereitet. Im Sinne der Entwicklungsverläufe interessieren vor allem die Veränderungen in den Einstellungen, die die pädagogischen Fach- und Lehrkräfte nach Besuchen der Fortbildungen der Initiative „Haus der kleinen Forscher“ in Bezug auf MINT-Themen und deren Vermittlung an Kinder berichten. „Da Überzeugungen eine hohe Stabilität aufweisen und sich selten von sich aus verändern, sind maßgeschneiderte Fortbildungen notwendig, damit Lehrkräfte ihre Überzeugungen weiterentwickeln“ (Kunina-Habenicht, Decker \& Kunter, 2016, S. 323). Vor allem die Reflexion der eigenen Überzeugungen, die z. B. in Fortbildungen angeregt wird, wird für diese Weiterentwicklung als wichtig angesehen, da sonst die bereits bestehenden Überzeugungen verhindern, dass Informationen aufgenommen werden, die den eigenen Vorstellungen widersprechen (Kunina-Habenicht, Decker \& Kunter, 2016). Daher sollte bedeutsam sein, worauf es den pädagogischen Fachund Lehrkräften besonders ankommt, wenn sie mit Kindern im MINT-Bereich forschen, was sie von den Fortbildungen der Initiative „Haus der kleinen Forscher“ erwarten und wie sie die Fortbildungen wahrnehmen.

\subsection{Motivation}

Wie aus dem oben beschriebenen Modell ersichtlich wird, sind motivationale Merkmale ein wichtiger Bestandteil der professionellen Kompetenz. Um Entwicklungsverläufe in der Professionalisierung von Fach- und Lehrkräften festzustellen, die an den Fortbildungen der Initiative „Haus der kleinen Forscher“ teilgenommen haben, ist daher die Betrachtung ihrer Motivation, MINT-Themen in den Kita-Alltag zu integrieren, unumgänglich.

Nach Kunter, Klusmann \& Baumert (2009) entscheiden berufsbezogene motivationale Merkmale, „inwieweit Personen sich engagieren, ihr Wissen tatsächlich anwenden oder sich weiterbilden“ (S. 157). Selbstwirksamkeitserwartung wird nach Kunter et al. $(2009,2011)$ und Steffensky et al. (2018) zu den motivationalen Merkmalen gezählt. Kunter et al. (2011) belegen, dass die Selbstwirksamkeitserwartung der pädagogischen Fach- und Lehrkräfte als Teil eines Systems motivationaler Orientierungen beschrieben und als ein Merkmal ihrer Motivation bezeichnet werden kann.

Zudem lässt sich zwischen intrinsischer und extrinsischer Motivation unterscheiden. Intrinsische Motivation ist eine Auswirkung des Verhaltens an sich. Sie liegt im Verhalten selbst, erfolgt zeitgleich und nicht zusätzlich. Sie bezieht sich also auf Motivation, die aus einer Aufgabe selbst entspringt, etwa weil diese als 
bedeutsam erscheint. Extrinsische Motivation dagegen speist sich aus den Ergebnissen eines Verhaltens und zusätzlichen Konsequenzen von außen. Extrinsische Motivation wirkt daher sozusagen aus zukünftig erwarteten Belohnungen (Glynn \& Koballa, 2006).

In diesem Projekt wird die Motivation von pädagogischen Fach- und Lehrkräften, MINT-Themen mit Kindern in der Kita und in der Grundschule anzugehen und an Fortbildungen der Initiative „Haus der kleinen Forscher“ teilzunehmen, unabhängig davon betrachtet, ob sie in- oder extrinsischer Natur ist. Somit werden beide Motivationsformen nicht getrennt erfasst. 


\section{Stand der Forschung}

Im Folgenden wird dargestellt, inwiefern Entwicklungsverläufe, vor allem in Hinsicht auf die Wirksamkeit formeller sowie informeller Lerngelegenheiten bisher empirisch untersucht wurden. Dies soll insbesondere bezogen auf die vorgestellten Kompetenzaspekte Professionswissen, Motivation und Einstellungen geschehen.

\subsection{Entwicklungsverläufe durch Nutzung von Lerngelegenheiten}

Das in Kapitel 2.1 vorgestellte „Modell der Determinanten und Konsequenzen der professionellen Kompetenz von Lehrkräften“ (siehe Abbildung 3) stellt Lerngelegenheiten sowie deren Nutzung für die Entwicklung der professionellen Kompetenz als relevant dar. Fraglich ist, ob ein Kompetenzzuwachs durch die tägliche Berufspraxis allein angenommen werden kann.

Brunner et al. (2006) können in ihrer Studie keinen systematischen Zusammenhang zwischen Berufserfahrung und den Facetten des professionellen Wissens feststellen und halten fest, dass davon ausgegangen werden kann, dass Berufs- und Unterrichtserfahrung alleine, also ohne die Nutzung strukturierter Lerngelegenheiten wie Fort- und Weiterbildungen, nicht automatisch zu einer Verbesserung des fachdidaktischen Wissens führen.

Aber auch die Nutzung von Lerngelegenheiten allein garantiert keinen Kompetenzzuwachs, wie ein Forschungsüberblick von Lipowsky und Rzejak (2012) zeigt. Sie setzten die Anzahl von besuchten Fortbildungen in Zusammenhang mit den Kompetenzen von Lehrkräften und kommen zu dem Ergebnis, dass Lehrkräfte, die häufiger an Fortbildungen teilnehmen, nicht per se kompetenter sind als ihre Kolleginnen und Kollegen. Daraus schließen sie, dass die Häufigkeit von Fortbildungsbesuchen allein kein genauer Indikator für die Unterrichtsqualität ist. Der Kompetenzzuwachs durch Fortbildungen hänge von verschiedenen Faktoren ab, beispielsweise von Merkmalen der Lernperson selbst, ihren Erwartungen, Zielen, ihrer Haltung gegenüber dem eigenen Lernen und dem Lernen der Schülerinnen und Schüler, ihren Vorkenntnissen, ihrer spezifischen Motivation, an der Fortbildung teilzunehmen sowie der Bereitschaft, die Fortbildungsinhalte in das eigene unterrichtliche Handeln zu integrieren. Auch kontextuelle Bedingungen wie die Konzeption und Gestaltung der Fortbildung spielten eine Rolle.

Welche Bedingungen eine Fortbildung genau erfüllen muss, um die Kompetenzentwicklung zu fördern, wurde in zahlreichen Studien, vor allem zur Wirkung von Lehrkräftefortbildungen, untersucht. Lipowsky und Rzejak (2012) nennen in 
ihrem Überblick Merkmale wie wissenschaftlich fundierter bereichsspezifischer Input, der längerfristig angelegt und durch Übungen und Anwendungen angereichert ist, sowie Phasen der Reflexion und des Austauschs mit Kolleginnen und Kollegen als bedeutsam für einen Kompetenzzuwachs. Darüber hinaus zeigen sie in ihrer Studie, dass die von Grundschullehrkräften eingeschätzte persönliche Relevanz der Fortbildung davon abhängt, ob die Fortbildung als Herausforderung erlebt wird, was wiederum die selbsteingeschätzte Verarbeitung der Fortbildungsinhalte und hierüber auch Veränderungen in Überzeugungen vorhersagen kann. Lipowsky und Rzejak (2012) schließen daraus, dass anspruchsvolle Fortbildungen als relevanter eingeschätzt und tendenziell besser verarbeitet werden.

Des Weiteren untersuchten Bosse et al. (2016) den Einfluss von Einstellungen auf die Beurteilung von Fortbildungen bei Lehrkräften. Sie kommen zu dem Ergebnis, dass das Erleben der Fortbildung sehr unterschiedlich beschrieben wird. Dabei scheinen die individuellen Einstellungen in starkem Zusammenhang mit den Bewertungen der Veranstaltungen zu stehen.

Neben der Qualität beziehungsweise wahrgenommenen Qualität der Fortbildung sind auch persönliche Merkmale der Fortbildungsteilnehmerinnen und -teilnehmer relevant. Vigerske (2017) stellt in ihrer empirischen Untersuchung zur Transferqualität und zu Einflussfaktoren zum Transfer von Lehrerfortbildungsinhalten fest, dass für die Übernahme von Fortbildungsinhalten die Motivation und ein gewisses Maß an Selbstwirksamkeit von besonderer Bedeutung sind. Lipowsky und Rzejak (2012) schlussfolgern in ihrem bereits erwähnten Forschungsüberblick, dass Lehrkräfte motiviert sind, an einer Fortbildung teilzunehmen, wenn diese in engem Bezug zu ihrer Praxis steht, in dem z. B. konkrete Anregungen und Material sowie Austausch mit Kolleginnen und Kollegen geboten werden. Richter, Richter und Marx (2018) untersuchten den Zusammenhang zwischen der Berufserfahrung von Lehrkräften und deren Motivation zur Teilnahme an Fortbildungen. Sie zeigen, dass Lehrkräfte mit mehr Berufserfahrung eher seltener Fortbildungen besuchen als Lehrkräfte mit weniger Berufserfahrung. Die Fortbildungsaktivität im Laufe des Berufslebens lässt sich laut Autorin und Autoren als umgekehrte U-Kurve beschreiben: Bis zur Mitte des Berufslebens lässt sich eine steigende Fortbildungsaktivität konstatieren, ehe sie mit zunehmendem Alter wieder sinkt. Berichtet wird weiter, dass Fortbildungen häufiger von Lehrkräften mit formaler Lehrbefähigung besucht werden. Mit Bezug auf die Schulform können zwar keine systematischen Unterschiede der Teilnahmequoten gefunden werden, doch Lehrkräfte an Gymnasien besuchten eher fachliche oder fachdidaktische Fortbildungen, während Lehrkräfte anderer Schularten eher Fortbildungen mit pädagogischen Inhalten aufsuchten. Diese Befunde zeigen, dass die individuelle Teilnahme der Lehrkräfte sowohl hinsichtlich der investierten Zeit als auch des besuchten Themas stark variiert. 
Bezüglich des Zusammenhangs zwischen den Kompetenzen von Lehrkräften und ihrer Teilnahme an Fortbildungen belegen Nitsche, Dickhäuser, Dresel und Fasching (2013), dass Lehrkräfte, die eine hohe Lernzielorientierung aufweisen und somit verstärkt nach der Erweiterung der eigenen Kompetenzen streben, häufiger an schulexternen Fortbildungen teilnehmen. Diese Ergebnisse weisen auf den interessanten Umstand hin, dass gerade solche Lehrkräfte besonders aktiv an Fortbildungen partizipieren, die sich bereits intensiver in der Schule engagieren und zumindest rein subjektiv ein höheres Kompetenzniveau besitzen (Richter, 2016).

\subsection{MINT-spezifische Studien zu Professionswissen, Motivation und Einstellungen}

Aktuelle Studien zu MINT-spezifischen Kompetenzen beim frühpädagogischen Personal setzen häufig bei dessen Fachwissen und seiner motivationalen Orientierung in Bezug auf MINT-Themen an. Die Befunde bestätigen sich größtenteils gegenseitig und zeigen ein eher verbesserungsbedürftiges Bild der vorhandenen Kompetenzen, wenn man die Frühförderung von Kindern in Mathematik, Informatik, Naturwissenschaften und Technik als wichtig erachtet (vgl. Appleton, 2003; Dunekacke, 2015; Garbett, 2003).

Laut Dunekacke (2015) gibt es durchaus große Unterschiede bezüglich des MINT-spezifischen Fachwissens bei pädagogischen Fachkräften in Deutschland. Zudem hätten viele „Angst“ oder „Hemmungen“, wenn sie naturwissenschaftliche Inhalte vermitteln sollten. Ihr Fachwissen in diesen Bereichen sei oft sehr viel schlechter als das von Grundschullehrkräften (Garbett, 2003), obwohl auch diese überwiegend eine negative Haltung und nur geringes Wissen im MINT-Bereich besäßen (Appleton, 2003).

Diese eher negative Bilanz steht also dem Ziel einer nachhaltigen MINT-spezifischen Frühförderung von Jungen und Mädchen aktuell entgegen. Ein Mittel zur Begegnung dieses Gegensatzes liegt in der Fort- und Weiterbildung der pädagogischen Fach- und Lehrkräfte in Bezug auf ihr eigenes Wissen in MINT-Themen sowie die didaktische Umsetzung von MINT-Inhalten. Studien mit Lehrkräften verdeutlichen, dass das professionelle Handeln als umso besser bezeichnet werden kann, je höher das einschlägige Wissen im MINT-Bereich ist (Baumert \& Kunter, 2006; Blömeke, Kaiser \& Lehmann, 2008; Kunter et al., 2013).

Entgegen der oben berichteten Ergebnisse von Lipowsky und Rzejak (2012) stellen Steffensky et al. (2018) im Rahmen der EASI-Science-Studie fest, dass sich Unterschiede hinsichtlich der Kompetenzen von pädagogischen Fachkräften abhängig von der Häufigkeit, naturwissenschaftliche Fortbildungen zu besuchen, ergeben. Fachkräfte, die in den letzten drei Jahren keine Fortbildung im natur- 
wissenschaftlichen Bereich besucht hatten, zeigten deutliche Unterschiede in den „motivationalen Kompetenzen (Interesse, Selbstkonzept, Selbstwirksamkeitserwartung und Enthusiasmus) sowie pädagogischen Überzeugungen (programmatischer Ansatz, kindzentrierter Ansatz, Open-Framework-Ansatz)“ (Steffensky et al., 2018, S. 131) zu Fachkräften, die in dieser Zeit mindestens zwei Fortbildungen

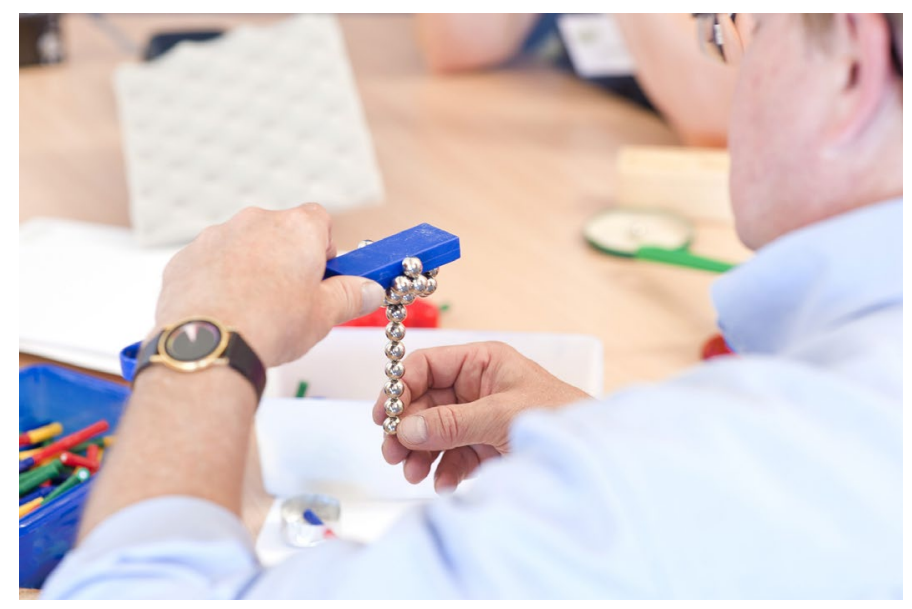
besucht hatten. Schon ein Jahr ohne Fortbildung bewirkte einen signifikanten Unterschied im Fachwissen zugunsten fortgebildeter Fachkräfte. Unterschiede im fachdidaktischen Wissen dagegen konnten nicht nur auf die Häufigkeit des Besuchs von Fortbildungen zurückgeführt werden, sondern sie liegen auch am sogenannten MINT-Hintergrund, wenn die Fach- und Lehrkräfte beispielsweise in der Ausbildung weitere naturwissenschaftliche Lerngelegenheiten hatten.

Um die oben benannten Ängste und Hemmungen abzubauen, kann neben der alleinigen Stärkung des Fachwissens ein zusätzlicher Ansatzpunkt sein, den Bezug der Fach- und Lehrkräfte zur Domäne der Naturwissenschaften zu stärken und diesen in Zusammenhang mit ihrem Fähigkeitsselbstkonzept einerseits und ihrem Fach- und fachdidaktischen Wissen andererseits zu bringen (Kauertz \& Gierl, 2014). Auch die MINT-Fortbildungen für Fach- und Lehrkräfte der Initiative „Haus der kleinen Forscher“ zielen neben der Steigerung des fachlichen und fachdidaktischen Wissens auf Motivation, Interesse und Fähigkeitsselbstkonzepte ab (Stiftung Haus der kleinen Forscher, 2013, 2015b, 2018, 2019).

\subsection{Forschungsdesiderate und daraus folgende Untersuchungsschwerpunkte}

Wie gezeigt wurde, können Studien einen positiven Einfluss auf die Entwicklung der professionellen Kompetenz durch Fortbildungen aufweisen, sofern diese Fortbildungen bestimmte Bedingungen erfüllen (vgl. Steffensky et al., 2018). Eine bedeutsame Rolle spielen dabei die Motivation, sich mit den Themen der Fortbildung zu beschäftigen, sowie die Selbstwirksamkeitseinschätzung in diesem Bereich. Nicht eindeutig geklärt ist dagegen, welche Rolle die Berufserfahrung oder die Anzahl der besuchten Fortbildungen spielt und ob Fach- und Lehrkräf- 
te in gleicher Weise von Fortbildungen profitieren, insbesondere wenn es um MINT-Themen geht. Noch nicht untersucht wurde, wie eine solche Kompetenzentwicklung verläuft und von welchen Faktoren sie abhängt. Um Fort- und Weiterbildungen im MINT-Bereich zu optimieren, erscheint es relevant, mehr über die Entwicklungsverläufe bei pädagogischen Fach- und Lehrkräften in Bezug auf die Kompetenz, mit Kindern zu forschen, zu erfahren. Dazu gehört auch die Erfassung von Hintergrundvariablen, die einen solchen Entwicklungsverlauf möglicherweise beeinflussen und eine Bedeutung für die Qualifizierungsbedarfe haben, wie beispielsweise die Ausbildung allgemein, eine MINT-spezifische Ausbildung, die Zugehörigkeit zu einer Berufsgruppe oder die Dauer der Berufserfahrung sowie die Einschätzung der Relevanz der besuchten Fortbildungen für die eigene professionelle Kompetenz, die sich auf professionelles Verhalten auswirken kann (siehe Abschnitt A 2.2). In der hier vorgestellten Studie soll dieses Forschungsdesiderat aufgegriffen und anhand einer explorativen Querschnittstudie mithilfe von Selbstauskünften von Fach- und Lehrkräften untersucht werden; eine Längsschnittstudie war aufgrund der Rahmenbedingungen nicht möglich. 


\section{Forschungsfragen}

Aus den beschriebenen Forschungsdesiderata und Untersuchungsschwerpunkten ergeben sich folgende über- und untergeordnete Forschungsfragen. Die zwei übergeordneten Forschungsfragen lauten:

FF1: Wie lassen sich die selbst wahrgenommenen Entwicklungsverläufe von pädagogischen Fach- und Lehrkräften in Bezug auf die Professionalisierung im MINTBereich beschreiben?

FF2: Gibt es verschiedene Phasen, Ereignisse oder typische Abläufe, die Fach- und Lehrkräfte für ihren Professionalisierungsprozess nennen?

Um diese beantworten zu können, werden im Vorfeld die folgenden Unterfragen betrachtet:

1. Wo stehen pädagogische Fach- und Lehrkräfte in Bezug auf forschendes Lernen mit Kindern nach ihrer eigenen Einschätzung? (FF U1)

2. Gibt es Unterschiede zwischen erfahrenen und weniger erfahrenen pädagogischen Fach- und Lehrkräften? (FF U2)

3. Gibt es Unterschiede zwischen pädagogischen Fachkräften und Grundschullehrkräften? (FF U3)

4. Wie lassen sich die Qualifizierungsbedarfe beschreiben? (FF U4) 


\section{Forschungsdesign und Stichprobenziehung}

Für die Studie wurde ein sequenzielles Mixed-Methods-Design gewählt (siehe Abbildung 6). Es handelt sich um ein sogenanntes Vorstudienmodell, bei welchem eine qualitative Studie einer quantitativen vorangestellt wurde (Döring \& Bortz, 2016). Nach Mayring (2001) ist dieses Design vor allem geeignet, wenn aus offen gestalteten Interviews Daten zur Operationalisierung von Indikatoren gewonnen werden sollen, die weiter zur Generierung eines geschlossenen Testinstruments genutzt werden (vgl. Ernst, 2004). Hier wurde eine qualitative Studie (Leitfadeninterviews) einer quantitativen Studie (Fragebogenerhebung) vorangestellt, um diverse Items zum Thema selbstwahrgenommene Entwicklungsverläufe für einen Online-Fragebogen auf Basis dieser Ergebnisse generieren zu können. Ziel der Interviews war es, zunächst Einblicke in die persönlichen Erfahrungen der Teilnehmerinnen und Teilnehmer mit dem Fortbildungsangebot der Initiative „Haus der kleinen Forscher“ zu gewinnen. Gleichzeitig sollten die Daten aus den Interviews auch wiederum zur Spezifizierung der Fragebogenergebnisse dienen. Dazu wurden die transkribierten Interviews mithilfe der qualitativen Inhaltsanalyse ausgewertet.

Der Online-Fragebogen wurde durch aus anderen Studien vorliegenden Einzelitems beziehungsweise Skalen ergänzt.

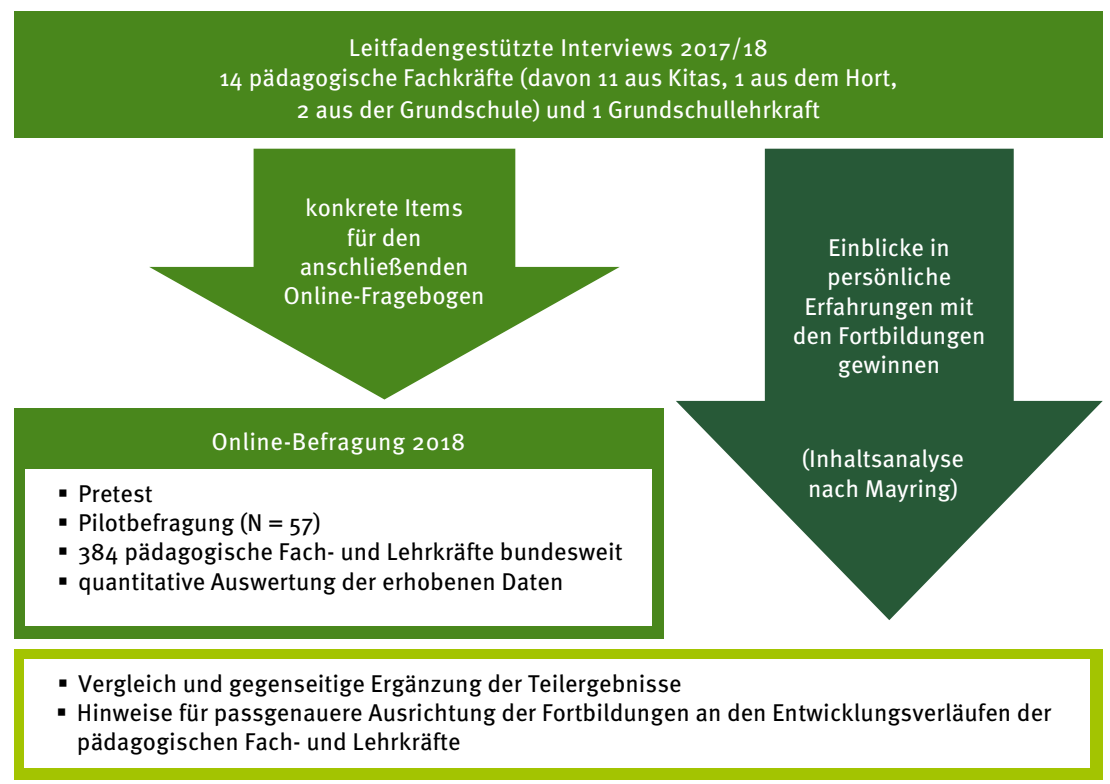

Abbildung 6. Forschungsdesgin 
Entwicklungsverläufe per se konnten mit diesem Design nicht gemessen werden, da im zeitlichen und finanziellen Rahmen des Projekts lediglich eine Querschnittbefragung möglich war. Stattdessen sollten mögliche Entwicklungsverläufe explorativ über Hilfskonstruktionen operationalisiert werden, indem erfahrene und weniger erfahrene Fach- und Lehrkräfte unterschieden wurden. Dies erfolgte aus Angaben zu Berufserfahrung, MINT-Hintergrund ${ }^{10}$ und der Anzahl an besuchten Fortbildungen. Außerdem sollten Fach- und Lehrkräfte aus dem Kita- wie aus dem Grundschulbereich einbezogen werden, um mögliche Unterschiede dieser Berufsgruppen in selbst berichteten Entwicklungsverläufen oder zu prägenden Ereignissen untersuchen zu können. Die erhaltenen quasimetrischen Daten wurden dazu mithilfe von mittelwertvergleichenden t-Tests und Varianzanalysen ausgewertet sowie durch Post-hoc-Analysen weiter aufgeklärt.

\section{Stichprobenziehung}

Für die Stichprobe hinsichtlich der Interviews wurde der Fokus auf eine bundesweite Auswahl von Fach- und Lehrkräften aus Kitas und Grundschulen gelegt, wobei sowohl zertifizierte als auch nicht zertifizierte Einrichtungen enthalten sein sollten; außerdem sollten Einrichtungen mit vielen und wenigen besuchten Fortbildungen bei der Initiative „Haus der kleinen Forscher“ berücksichtigt werden (vgl. Steffensky et al., 2018).

Die Stichprobenziehung für die Online-Befragung folgte der Logik einer Zufallsauswahl aus einem durch die Stiftung „Haus der kleinen Forscher“ zur Verfügung gestellten Pool. Bei diesem Pool handelte es sich um eine proportionale Stichprobe aus Einrichtungen, die zuvor bereits an Fortbildungen oder der Zertifizierung der Stiftung „Haus der kleinen Forscher“ teilgenommen hatten und bei der der Anteil an Kindergärten, Grundschulen und Horten so verteilt war, wie er in der Datenbank (Grundgesamtheit) der Stiftung „Haus der kleinen Forscher“ abgebildet ist. Innerhalb der Gruppen der Kitas, Horte und Grundschulen wurden die Fälle dann zufällig gezogen. Insgesamt wurden 3.000 Einrichtungen durch die Forschungsgruppe angeschrieben. 384 Fragebögen wurden beantwortet. Die Rücklaufquote war mit rund 13 Prozent eher gering, was mit der Länge des Fragebogens zusammenhängen könnte. Es können keine Rückschlüsse dazu gezogen werden, aus wie vielen Einrichtungen die Befragten stammen, um eine mögliche Verzerrung der Stichprobe durch die Teilnahme mehrerer Fach- oder Lehrkräfte aus einer Einrichtung zu kontrollieren.

10 Dies bedeutet, dass die Fach- und Lehrkräfte naturwissenschaftliche Lerngelegenheiten z. B. in der Ausbildung hatten. 


\section{Instrumentenentwicklung und Durchführung}

Das Vorgehen der Fragebogenerstellung bei der Umsetzung des eben aufgeführten Forschungsdesigns wird in vier Schritten beschrieben: Entwicklung des Interviewleitfadens, Ableitung von Items aus der Interviewbefragung, Modellierung der Items zu Entwicklungsverläufen und Pilotierung des Online-Fragebogens.

\subsection{Interviewleitfaden}

Die Interviews wurden, wie oben aufgeführt, mithilfe eines leitfadengestützten Designs (Flick, 2006) durchgeführt. Dabei war es wichtig, die Fragen verständlich zu formulieren und an die Erfahrungen der Probandinnen und Probanden anzuknüpfen sowie mithilfe der Fragen Raum für subjektive Schilderungen zu schaffen. Ausgehend von der theoretischen Herleitung und dem Forschungsdesign entsprechend wurde der Leitfaden für die Interviews entwickelt. Die ersten beiden Fragen prüfen Hintergrundvariablen ab, die eine erste Unterscheidung der Aussagen nach Berufsgruppen ermöglichen. Die dritte und vierte Frage zielen auf das potenzielle professionelle Verhalten der Befragten ab.

Die fünfte und sechste Frage sollen der Erfassung von Einstellungen zu MINTThemen dienen. Der Fragenkomplex von der siebten bis zur 17. Frage dreht sich um die Teilnahme an den Fortbildungsveranstaltungen. Die Fragen 18 bis 20 sollen erfassen, was nach der Fortbildungsteilnahme in den Einrichtungen durchgeführt wurde. Frage 21 fragt nach der Nutzung der Online-Angebote. Die 22. Frage lässt Raum für Wünsche und die 23. fragt nach der Weiterempfehlung an Kolleginnen und Kollegen. Aus dem letzten Fragenkomplex sollen sich Rückschlüsse auf die Motivation für weitere Teilnahmen und die fortführende Beschäftigung mit MINT-Themen ziehen lassen.

Der Leitfaden für die durchgeführten Interviews setzt sich aus den folgenden Fragen zusammen:

1. Sind Sie Fach- oder Lehrkraft?

2. Ist Ihre Kita/Grundschule von der Stiftung „Haus der kleinen Forscher“ zertifiziert?

3. Bild 1: Nach einem Waldspaziergang mit den Kindern, bei dem es zu kalt war, um am Wasserloch zu matschen, stellt die Fachkraft eine Lernumgebung her, in der die Kinder sowohl mit Wasser als auch mit anderen in der Natur vorkommenden Materialien experimentieren können. Was geht Ihnen durch den Kopf, 
wenn Sie diese Situation sehen? Was würden Sie als pädagogische Fachkraft/ Grundschullehrkraft hier tun?

4. Bild 2: Kinder versuchen beim Spielen im Freien einen abgesägten Baumstamm aufzurichten. Da dieser sehr schwer und unhandlich ist, geht es nicht alleine. Da müssen sich die Kinder etwas einfallen lassen. Was geht Ihnen durch den Kopf, wenn Sie diese Situation sehen? Was würden Sie als pädagogische Fachkraft/Grundschul-Lehrkraft hier tun?

5. Welche Bedeutung messen Sie den MINT-Themenbereichen für frühkindliche Bildung bei? Ist es wichtig, Kinder bereits frühzeitig an mathematische, informatische, naturwissenschaftliche und technische Themen heranzuführen, oder sehen Sie das eher kritisch?

6. Wieso haben Sie sich entschlossen, an einer oder mehreren Fortbildungen der Initiative „Haus der kleinen Forscher“ teilzunehmen? Wie kam es dazu?

7. Können Sie sich erinnern, zu welchem Zeitpunkt Sie Ihre erste, zweite, dritte usw. Fortbildung besucht haben und welche das waren?

8. Schildern Sie doch mal, wie eine Fortbildung im „Haus der kleinen Forscher“ typischerweise abläuft!

9. Wenn Sie an Ihre allererste „Haus der kleinen Forscher“-Fortbildung denken, was ist Ihnen besonders in Erinnerung geblieben?

10. Hat Sie die erste Fortbildung zur Teilnahme an weiteren motiviert? Warum (nicht)?

11. Woran erinnern Sie sich noch in Bezug auf die weiteren von Ihnen besuchten Fortbildungen?

12. Gibt es spezielle Ereignisse in oder nach den Fortbildungen, die Sie besonders (de-)motiviert haben? Warum?

13. Welche Inhalte der Fortbildungen waren besonders spannend für die Kinder?

14. Was konnten Sie nach den Fortbildungen beim Forschen mit den Kindern gleich gut im Kindergarten/in der Schule umsetzen und was nur schwer oder gar nicht?

15. Was genau haben Sie durch die Fortbildungen dazugelernt?

16. Wie hat sich durch die Fortbildungen Ihre Einstellung zum Forschen mit Kindern verändert?

17. Inwieweit beeinflussen die Fortbildungen Ihr Bild von einer guten pädagogischen Fach- beziehungsweise Lehrkraft?

18. Stellen Sie eine Wirkung der Fortbildungen auf Ihr Verhalten in der Einrichtung fest? In welcher Form?

19. Erfolgt nach einer Fortbildung ein reflektierender Austausch über die Inhalte mit Kolleginnen und Kollegen? (Teamarbeit?) Jedes Mal/teilweise? Wie genau erfolgt der Austausch? Worüber wird gesprochen? 
20. Was wurde im Nachhinein von Ihren Kolleginnen oder Kollegen bezüglich der Fortbildungsinhalte zurückgemeldet?

21. Haben Sie bisher auch Online-Angebote zur Fortbildung genutzt? Welche? Wie beurteilen Sie sie im Vergleich zu den Präsenzfortbildungen?

22. Welche Inhalte hätten Sie sich in den Fortbildungen gewünscht, die nicht thematisiert wurden?

23. Wie würden Sie eine Kollegin oder einen Kollegen motivieren, an solchen Fortbildungen teilzunehmen?

\subsection{Ableitung von Items aus der Interviewbefragung}

Um relevante Items für den Online-Fragebogen aus den geführten Interviews abzuleiten, war es notwendig, die Interviews zunächst zu transkribieren und bei der Sichtung des Datenmaterials stets die zentralen Forschungsfragen vor Augen zu haben.

Für einen ersten Überblick über das in den Interviews gewonnene Stimmungsbild der Fortbildungsteilnehmerinnen und -teilnehmer wurden einzelne prägnant erscheinende Aussagen in argumentativer Validierung (Döring \& Bortz, 2016) durch die Forschungsgruppe ausgewählt, die die Eindrücke der Fach- und Lehrkräfte angemessen abbilden und zusammenfassen:

- Frühförderung MINT wird als wichtig erachtet: „Also ich finde schon, dass man so mit zwei spielerisch mit MINT anfangen sollte! Dadurch werden die Wahrnehmung und die Sinne gestärkt, dass die das alles viel mehr wahrnehmen. “

- Das bisherige Fortbildungsformat wird überwiegend positiv bewertet: „Man muss das ja auch nicht alles immer wissenschaftlich begründen. Sondern es geht darum, das Forschen an sich anzuregen. Ums Ausprobieren. “

- Die Fortbildungen würden Theorie mit Praxis verbinden und die Fach- und Lehrkräfte darin schulen, kindgerecht zu MINT-Themen zu forschen: „Dass die Kinder selber ihre Hypothesen aufstellen. Das ist auch immer das Spannende! Dass sie auf eigene Ideen kommen!“

- Die Fortbildungen werden als spannend, kurzweilig und praxisnah erlebt: „Und man kann staunen, und wir wollten auch unsere Kinder zum Staunen bringen. Das heißt, der Workshop hat uns sofort mitgerissen!“

- Die Fortbildungen scheinen die Häufigkeit des Forschens mit Kindern in Projekten, Beobachtungen, Experimenten oder selbst Gebautem zu erhöhen: „Ja, dass man nach den Fobis unmittelbar ganz viel forscht und ehm versucht, viel Zeit dafür freizumachen. “ 
- Das selbstständige Ausprobieren wird als prägendes Ereignis aus der Fortbildung beschrieben: „Überhaupt, dass man was ausprobieren darf, forscht, das finde ich nun sehr wichtig!“

- Die Forschungsaktivitäten, die im Rahmen der Fortbildungen als Beispiele durchgeführt wurden, ließen sich leicht mit den Kindern wiederholen: „Alles, was ich selbst probiert hatte, das konnte ich gleich sehr gut umsetzen.“

- Positiv bewertet werden auch die über die Fortbildung hinausgehenden Online-Angebote, Literaturempfehlungen und die von den pädagogischen Fachkräften so benannte „Nach-Hause-Tüte“, die pädagogischen Materialien und Anregungen, hier im Speziellen die Forscherkarten und der Forscherbus: „Es ist klar geworden, dass man mit den allereinfachsten Materialien Phänomene untersuchen kann.“

Nach Fertigstellung des Kategoriensystems (siehe Abschnitt A 7.1.2) konnten relevante Aspekte der Fortbildungen für die Fragestellung zum einen über die Häufigkeit ihrer Nennung bestimmt werden, zum anderen über die Diversität der gegebenen Antworten. Der durch die erhobenen Interviews gewonnene Einblick in das Fortbildungsformat erlaubte spezifische Erkenntnisse über Ablauf, Schwerpunkt und Intention der Fortbildungen und führte so zu konkreten Items, die schließlich in den Online-Fragebogen Einzug fanden. Die hervorgehobenen Formulierungen dienten u. a. als Anregung für Fragebogenitems. Die konkreten Items, die aus den Interviews generiert wurden, sind folgende:

- Item 1.7 und 1.8, die nach der Zertifizierung der Einrichtungen und dem MINTHintergrund der pädagogischen Fach- und Lehrkräfte fragen.

- Item 2.4 bis 2.8, die erfragen, worauf es den Fach- und Lehrkräften beim Forschen mit Kindern besonders ankommt.

- Item 4.7 und 4.8, welche das professionelle Verhalten von pädagogischen Fach- und Lehrkräften thematisieren.

- Item 5.1 und 5.7 bis 5.9 sowie 5.12, die Erwartungen an die Fortbildungen, deren Einfluss auf die Einstellungen zu MINT-Themen und das Forschen mit Kindern abbilden.

- Item 6.8 bis 6.17, die danach fragen, was in der Fortbildung konkret gelernt wurde.

- Item 6.18, das erfasst, welche Merkmale der Fortbildung als besonders empfunden werden. In Verbindung mit Item 8.25 geht es hier auch darum, inwiefern das selbstständige Entdecken und Forschen als prägendes Ereignis für die eigene professionelle Entwicklung eingeschätzt wird. 
- Item 7.3, das erhebt, ob die Fortbildungsinhalte auch an Kolleginnen und Kollegen kommuniziert werden.

- Item 8.16 bis 8.18, als Ergänzungen zu den Phasen des „Forschungskreises“ (siehe Abschnitt A 6.3.1; vgl. Marquardt-Mau, 2004, 2011; Rank et al., 2018; Stiftung Haus der kleinen Forscher, 2013).

- Item 8.25, das besonders hilfreiche Aspekte aus den Fortbildungen thematisiert.

Item 11.1, das Wünsche der Teilnehmerinnen und Teilnehmer erfasst.

\subsection{Modellierung der Items zu Entwicklungsverläufen}

Die Frage nach den Entwicklungsverläufen der pädagogischen Fach- und Lehrkräfte in ihrer Professionalisierung steht an zentraler Stelle dieses Projekts.

Insgesamt werden in der vorliegenden Studie drei Kompetenzfacetten erfasst: Motivation, Einstellungen sowie das Professionswissen mit dem Schwerpunkt auf fachdidaktischem Wissen und potenziellem professionellem Verhalten. ${ }^{11}$ Gefragt wurde jeweils nach dem selbsteingeschätzten aktuellen Stand der Motivation, der Einstellungen und des Professionswissens sowie nach der selbst wahrgenommenen Veränderung durch den Besuch der Fortbildungen.

Die Verläufe wurden aus der Sicht der Teilnehmerinnen und Teilnehmer erfasst, es handelt sich also um selbsteingeschätzte Verläufe, die von außen wahrgenommenen oder gemessenen Entwicklungen abweichen können und ggf. im Selbstbericht eher positiver ausfallen. Sozial erwünschtes Antwortverhalten wurde nicht überprüft und kann deshalb nicht ausgeschlossen werden.

Neben den aus den Interviews abgeleiteten Items wurden Items aus verschiedenen schon vorhandenen Instrumenten zusammengestellt. Die Quellen sind im Folgenden in Klammern angegeben. Einige Items wurden für die spezifische Fragestellung der vorliegenden Studie erweitert. Diese Änderungen wurden im Vorhinein argumentativ (Döring \& Bortz, 2016) und nach der Pilotierung statistisch validiert.

\subsubsection{Selbsteingeschätztes Professionswissen}

Bezugnehmend auf die Fortbildungen der Initiative „Haus der kleinen Forscher“ war zunächst zu fragen, welches Professionswissen die Fach- und Lehrkräfte durch das Fortbildungsangebot vermittelt bekamen. Anhand der Interviews stellte sich

11 Andere Teilaspekte des Professionswissens wurden nicht erfasst. Dies waren Bestandteil der EASIScience-Studie (Steffensky et al., 2018, S. 99). 
heraus, dass der „Forschungskreis“ als Hilfestellung für Fachund Lehrkräfte beim Forschen zu MINT-Themen mit Kindern als fachdidaktisches Wissen betrachtet werden kann (vgl. Marquardt-Mau, 2004, 2011; Rank et al., 2018; Stiftung Haus der kleinen Forscher, 2013).

Um Professionalisierungsverläufe zu erfassen, war der Aspekt der temporären Veränderung mit aufzunehmen; demzu-

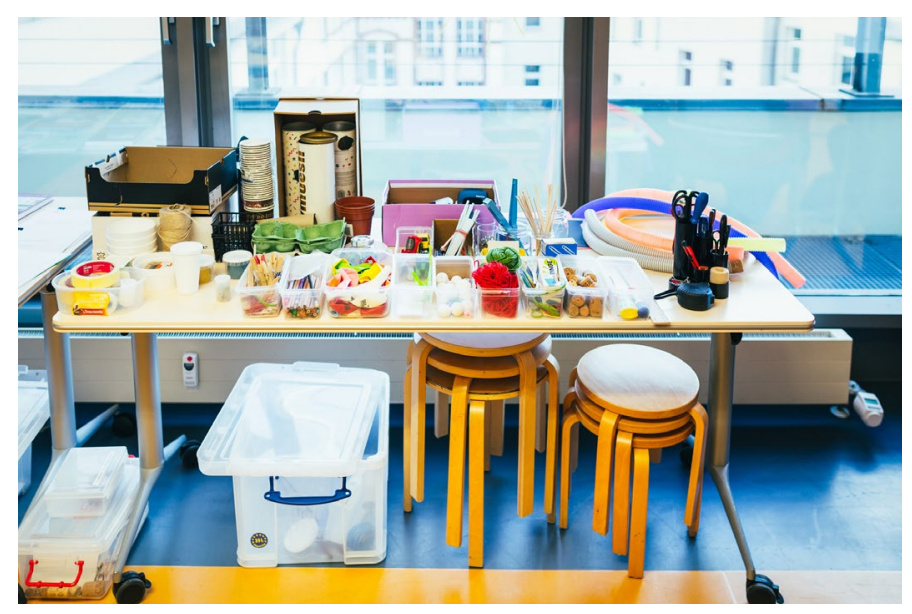
folge lauteten die Fragen hierzu:

„Bitte schätzen Sie Ihre Kompetenz zum Forschen mit Kindern in den folgenden Bereichen jeweils zum Zeitpunkt direkt nach der Ausbildung, nach der ersten Fortbildung und nach der letzten Fortbildung auf einer Skala von 1 (=sehr schlecht) bis 10 (=sehr gut) ein (Item 8.1-8.21):

1. Interessen der Kinder erkennen

2. Vorkenntnisse der Kinder einschätzen

3. Experimente vorbereiten

4. Anregende Fragen stellen, ohne die Antwort zu verraten

5. Ideen der Kinder aufgreifen

6. Kindgerechtes Erklären von Naturphänomenen

7. Dokumentieren von Experimenten und Ergebnissen.“

Ergänzt wurde der Aspekt „Kindgerechtes Erklären von Naturphänomenen“, weil diese Umschreibung in den Interviews gewählt wurde, wenngleich es das Ziel der Stiftung „Haus der kleinen Forscher“ eher ist, die Kinder bei eigenen Erklärungen und dem eigenen Verstehen zu unterstützen.

Zusätzlich haben wir das selbsteingeschätzte fachdidaktische Wissen der Befragten über sechs Items (4.1-4.6) erhoben, wobei zu je einem Phänomen abgefragt wurde, inwiefern sich zugetraut wird, den entsprechenden Beispielsachverhalt Kindern erklären zu können (NFFK-Inventar nach Zimmermann, 2011, nach eigenem Ermessen abgewandelt).

Das potenzielle professionelle Verhalten wurde wie schon in den Interviews anhand zweier Beispielsituationen (Item 4.7-4.16) erfasst, bei denen jeweils dazu 
Stellung genommen werden sollte, was man persönlich in einer solchen Situation tun würde (aus Interviews abgeleitet und nach eigenem Ermessen ergänzt).

Bezüglich der wahrgenommenen Wirksamkeit der Fortbildungen haben wir weitere 15 Items $(6.1,6.3,6.5,6.6,6.8-6.17,6.19)$ verwendet, um zu erfassen, ob die Befragten den Eindruck haben, dass die Fortbildungen sie auf die Rolle als Lernbegleitung vorbereiten, ihr MINT-Wissen verbessern, sich auf den Forscherdrang der Kinder auswirken und Wichtiges für die tägliche Arbeit in den Einrichtungen vermitteln. Zudem wurde danach gefragt, was konkret in den „Haus der kleinen Forscher"-Fortbildungen gelernt wurde und ob dies dazu beigetragen hat, vertiefte Denkprozesse bei den Kindern anzustoßen (aus Interviews abgeleitet und nach eigenem Ermessen ergänzt).

\subsubsection{Selbsteingeschätzte Motivation}

Um die Motivation der pädagogischen Fach- und Lehrkräfte im Sinne des Kompetenzmodells (Kunter et al., 2011) in Bezug auf das Forschen mit Kindern zu MINTThemen zu ermitteln, wurden 12 Items entwickelt, welche orientiert am Monitoring-Bericht 2016/2017 der Stiftung „Haus der kleinen Forscher“ entstanden sind (Stiftung Haus der kleinen Forscher, 2017).

Zunächst wurde danach gefragt, ob prinzipiell die Bereitschaft besteht, sich auch in der eigenen Freizeit mit MINT-Themen zu beschäftigen (Item 2.3, Item nach eigenem Ermessen ergänzt). Danach wurden Items zur Erfassung der Selbstwirksamkeitserwartung aufgeführt (Item 3.1 bis 3.6, mit kleinen Veränderungen z. B. in Antwortmöglichkeiten aus Monitoring-Bericht 2016/2017, Stiftung Haus der kleinen Forscher, 2017). Auch die Frage, ob sich nach den Fortbildungen die Häufigkeit des Forschens erhöht hat (Item 6.4), wurde gestellt. Zudem wollten wir wissen, ob aktuell die Motivation besteht, an weiteren MINT-Fortbildungen teilzunehmen (Item 7.1) und wie viele Forschungsaktivitäten aus den Fortbildungen (im Folgenden sogenannte Beispielexperimente) bereits mit Kindern wiederholt wurden (Item 7.4). Abschließend war von Interesse, zu welchen Themen bereits geforscht wurde (Item 8.22) und wie häufig in der eigenen Einrichtung zu MINTFragestellungen geforscht wurde (Item 8.23, alle mit kleinen Veränderungen aus dem Monitoring-Bericht 2016/2017 der Stiftung „Haus der kleinen Forscher“, Stiftung Haus der kleinen Forscher, 2017 ergänzt).

\subsubsection{Einstellungen}

Um die Einstellungen der Befragten in Anlehnung an das COAKTIV-Kompetenzmodell (Kunter et al., 2011) in Bezug auf MINT-Themen und deren Vermittlung an Kinder zu erfassen, wurden zehn Items gebildet. Es wurde abgefragt, inwiefern der Förderbereich MINT für wichtig in Kitas und Grundschulen erachtet wird (Item 
2.1 und 2.2, vgl. Steffensky et al., 2018) und worauf es den Befragten besonders ankommt, wenn sie mit Kindern zu MINT-Themen forschen (Item 2.4 bis 2.8, aus Interviews abgeleitet). Zusätzlich war von Interesse, ob die MINT-Themen nach einem Fortbildungsbesuch der Initiative „Haus der kleinen Forscher“ für interessanter gehalten werden als vorher (Item 5.7, aus Interviews abgeleitet). Daneben erhoben wir, ob die Fortbildungen dazu beitragen, die eigene Meinung zur frühkindlichen Auseinandersetzung mit MINT-Themen zu verändern (Item 5.8, aus Interviews abgeleitet) und ob den Befragten das Forschen zu MINT-Themen zunehmend leichter fällt (Item 5.9, aus Interviews abgeleitet).

\subsection{Pilotierung des Fragebogens}

Auf Grundlage der in den Interviews gewonnenen Eindrücke und der, wie in $A b$ schnitt A 6.3 dargestellt, entliehenen Items, wurde ein Online-Fragebogen entwickelt. Es folgte ein Pretest mit sechs ehemaligen Interviewpartnerinnen und -partnern, die sich dazu bereit erklärt hatten. Es ging hier vor allem um die Beseitigung von missverständlichen Formulierungen, das Testen der Übersichtlichkeit des Fragebogens und die Ermittlung eventueller Schwierigkeiten bei der Beantwortung der Fragen. Nach einer entsprechenden Überarbeitung des Instruments erfolgte eine Pilotbefragung von insgesamt 57 pädagogischen Fach- und Lehrkräften (45 pädagogische Fachkräfte, zehn Lehrkräfte, zwei ohne Auskunft). Erfragt wurde dabei nicht, ob die pädagogischen Fachkräfte im Kindergarten- oder im Hortbereich arbeiten. Diese Antwortdifferenzierung wurde in der Hauptuntersuchung ergänzt.

Ziel der Pilotierung war es, vor allem aufgetretene Varianzen im Antwortverhalten zu ermitteln, um so Items mit wenig Varianz ausschließen und den Fragebogen entsprechend kürzen zu können, eventuell auftretende Schwierigkeiten in der Beantwortung einzelner Frage zu identifizieren sowie die Güte verschiedener Fragenblöcke zu erfassen. Bei der Pilotierung traten keine Schwierigkeiten bei der Beantwortung auf, aber der Bogen wurde als zu lang empfunden. Deshalb war die Kürzung des Fragebogens notwendig.

Insgesamt bestand der Pilotfragebogen aus elf übergeordneten Skalen:

1. Hintergrundvariablen

2. Einstellungen zu MINT-Themen

3. Persönlichkeitsmerkmale

4. aktuelle Frühförderkompetenz im MINT-Bereich

5. besuchte Fortbildungen der Initiative „Haus der kleinen Forscher“

6. Einschätzung der Wirksamkeit dieser Fortbildungen 
7. Einflussfaktoren auf Entwicklungsverläufe

8. Entwicklungsverläufe

9. die letzte Forschungsaktivität mit Kindern

10. Genderaspekte

11. Wünsche der Teilnehmerinnen und Teilnehmer

Die Güte der einzelnen Skalen wurde separat überprüft. Dazu wurden Reliabilitätsprüfungen mittels Cronbachs Alpha durchgeführt, was ein Maß für die interne Konsistenz der Skalen darstellt. Sinnvoll angewendet werden konnte diese Überprüfung allerdings nur bei Skalen mit mindestens ordinalem Skalenniveau. Konkret bedeutete dies, dass die Reliabilität im Falle der Selbstwirksamkeitserwartung ( $\alpha=0,759)$ (Skala 3), des Selbstkonzepts $(\alpha=0,838)$ (Skala 3), des fachdidaktischen Wissens $(\alpha=0,748)$ (Skala 4), der Wirksamkeit der Fortbildungen ( $\alpha=0,651$ ) (Skala 6) und der Entwicklungsverläufe $(\alpha=0,919)$ (Skala 8) überprüft, festgestellt und mit gut bis zufriedenstellend bewertet werden konnte.

Aufschluss darüber, welche Items aus dem Instrument gestrichen werden konnten, gab zudem die Betrachtung der Varianz innerhalb eines Items (Ausschluss von Items mit besonders geringer Varianz) sowie die systematisch inhaltliche Überlegung, welche Items zur Beantwortung der Forschungsfragen beitrugen. Zudem gab es einige Items, die Ähnliches abbildeten und somit gestrichen werden konnten.

Neben dem Kürzen des Bogens wurden die Antwortkategorien vereinheitlicht. So wurden beispielsweise alle Skalierungen auf eine 4er-Skalierung festgelegt (bis auf die der Kompetenzverläufe) und die Ausrichtung von Antworten mit ordinalem Messniveau stets von kleineren zu größeren Werten bestimmt.

Zum Schluss der Pilotierung wurden noch Items mit aufgenommen, die Erkenntnisbedarfe der Stiftung „Haus der kleinen Forscher“ zur etwaigen (Weiter-) Entwicklung ihrer Angebote aufgriffen. Sie adressierten vor allem Genderaspekte (Skala 10). Der Umfang des Fragebogens reduzierte sich durch die Pilotierung von 164 auf 114 Items.

Die eigentliche Erhebung fand dann unter der freiwilligen Teilnahme von insgesamt 384 pädagogischen Fach- und Lehrkräften (Stichprobenbeschreibung siehe Abschnitt A 7.2.1) statt. Sie wurde online durchgeführt, um eine bundesweite Beteiligung zu ermöglichen. 


\section{Auswertung}

In diesem Kapitel wird das Vorgehen zur Auswertung der beiden eingesetzten Befragungen (qualitative und quantitative Befragung) dargestellt sowie die jeweiligen Stichproben beschrieben. Im ersten Teilkapitel zur qualitativen Befragung wird nach der Beschreibung der Stichprobe die Verarbeitung der Interviewdaten und deren Auswertung beschrieben. Im zweiten Teilkapitel zur quantitativen Befragung erfolgt nach der Stichprobenbeschreibung die Beschreibung der statistischen Auswertung des Online-Fragebogens.

\subsection{Interviews}

Die Interviews wurden leitfadengestützt durchgeführt (siehe Abschnitt A 6.1) und erfolgten telefonisch, sodass die Teilnehmerinnen und Teilnehmer über das ganze Bundesgebiet verteilt sein konnten. Insgesamt wurden 15 Interviews mit pädagogischen Fach- und Lehrkräften durchgeführt, aufgezeichnet und transkribiert. Alle Interviews konnten für die Auswertung berücksichtigt werden.

\subsubsection{Stichprobe}

Es wurden 14 pädagogische Fachkräfte ${ }^{12}$ sowie eine Grundschullehrkraft befragt. Es befand sich ein Mann unter den Befragten. Ihre jeweiligen Einrichtungen waren in zehn Fällen bereits von der Stiftung „Haus der kleinen Forscher“ zertifiziert. Bei den anderen fünf war dies nicht der Fall, laut eigener Aussage war aber eine Zertifizierung in Planung.

Fünf der befragten Personen waren aus Nordrhein-Westfalen, vier Personen aus Baden-Württemberg, drei Personen aus Sachsen, zwei Personen aus dem Saarland und eine Person aus Brandenburg.

In vier Fällen wurden bisher bis zu drei Fortbildungen besucht. Acht der Interviewten hatten bereits bis zu sechs Fortbildungen besucht. Zwei Personen wiesen bis zu neun Fortbildungen auf und eine Person hatte bereits an zwölf Fortbildungen teilgenommen. Durchschnittlich hatten die Fachkräfte fünf Fortbildungen besucht. Die Lehrkraft hatte bisher sechs Fortbildungen besucht.

12 Davon arbeiteten zwei an einer Grundschule und eine an einem Hort. Die weiteren elf pädagogischen Fachkräfte arbeiteten in Kindertagesstätten. 


\subsubsection{Datenanalyse der Interviews}

Die Interviews wurden mithilfe der qualitativen Inhaltsanalyse von Mayring (2002) ausgewertet. Dazu wurden sie zunächst transkribiert und anschließend im Programm MaxQDA mithilfe eines Kodiersystems systematisch kodiert.

Das Kategoriensystem wurde deduktiv und induktiv erstellt. In einem ersten Schritt wurden in Anlehnung an das Kompetenzmodell von COAKTIV (Kunter et al., 2011; siehe Abschnitt A 2.1) deduktiv sogenannte Hauptkategorien gebildet: Professionswissen, Motivation und Einstellungen zu MINT-Themen. Den Hauptkategorien wurden in einem ersten Schritt die passenden Analyseeinheiten aus den Interviews zugeordnet. Im Anschluss wurden diese Einheiten in der Forschungsgruppe diskutiert und Subkategorien herausgebildet. In allen drei Hauptkategorien ließen sich Aussagen zu den folgenden vier Themenblöcken identifizieren: MINT-Themen allgemein, Forschen mit Kindern, Fortbildung und Material (siehe Abbildung 7).

\section{Professionelle Kompetenz}

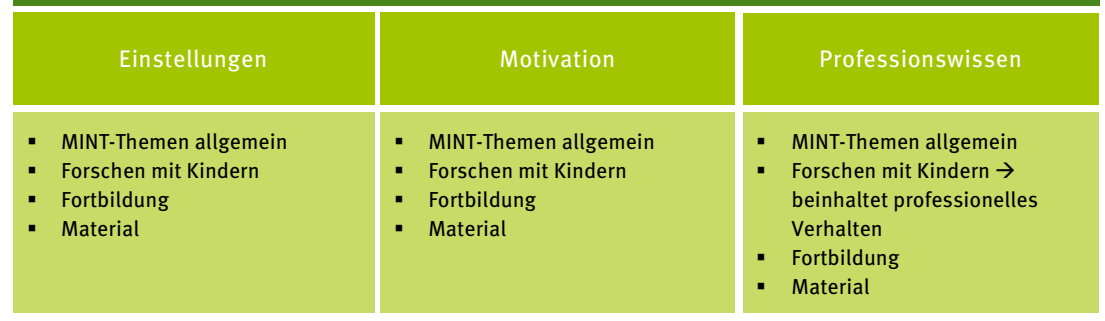

Abbildung 7. Einordnung der Interviewdaten in Themenfelder (eigene Darstellung)

Die einzelnen Analyseeinheiten, nämlich die Aussagen der Befragten, wurden dann den Themenblöcken zugeordnet. Die so zugeordneten Analyseeinheiten wurden in einem weiteren Schritt mithilfe induktiv gewonnener Subkategorien wie z. B. „Aufgaben einer pädagogischen Fachkraft“ (siehe Anhang) kodiert. Auf diese Weise wurden auch Aussagen aufgenommen, nach denen nicht explizit gefragt worden war.

Wie im zweiten Kapitel beschrieben, fließen die Aspekte Lerngelegenheiten, Nutzung von Lerngelegenheiten, professionelles Verhalten und persönliche Voraussetzungen mit ihren durch die Interviews zu erfassenden Elementen mit ein. Die Fortbildung als Lerngelegenheit ist in jeder Hauptkategorie als eigener Themenblock berücksichtigt, hier werden vordergründig die Fortbildung bewertende Aspekte erfasst. Die Interviews erfassen aber auch mögliche Auswirkungen der Fortbildung auf Kompetenzentwicklungsverläufe, sodass diese Teilaspekte im Kategoriensystem unter dem Aspekt professionelle Kompetenz berücksichtigt wurden. Die Teilnahme an der Fortbildung sowie das Auseinandersetzen mit den 
zur Verfügung gestellten Materialien im Anschluss an die Fortbildung werden als Nutzung von Lerngelegenheiten angesehen. Eine Nutzung von Lerngelegenheiten manifestiert sich in der professionellen Kompetenz. Daher wurden die Aspekte, die mit der Nutzung der Lerngelegenheit (Fortbildung der Initiative „Haus der kleinen Forscher") zusammenhängen, in die Hauptkategorie professionelle Kompetenz eingeordnet. Der Aspekt des professionellen Verhaltens wird insbesondere in der Kategorie Professionswissen im Themenblock Forschen mit Kindern berücksichtigt. Die persönlichen Voraussetzungen der pädagogischen Fach- und Lehrkräfte wurden in den Interviews nicht explizit erfragt. Die persönliche Motivation wurde in einigen Aspekten jedoch deutlich, sodass die Aussagen in diesen Themenbereich einfließen.

Das vollständige Kategorien- und Kodiersystem befindet sich im Anhang. Das Vorgehen orientierte sich am Ablaufmodell nach Mayring (2002); die Kodierungen wurden von einer zweiten Person gegenkodiert. Nicht-Übereinstimmungen wurden kommunikativ validiert.

In der Auswertung wurden die genannten Aussagen nicht fallbezogen, sondern anhand der Kategorien strukturiert und quantifiziert. Der Interpretationsfokus lag auf der selbst wahrgenommenen Entwicklung der professionellen Kompetenz der pädagogischen Fach- und Lehrkräfte, inklusive ihrer Motivations-, Einstellungs- und Wissensentwicklung.

\subsection{Online-Befragung}

In diesem Kapitel erfolgt zunächst eine Beschreibung der Stichprobe der Online-Befragung. Dann wird das Vorgehen bei der Auswertung der Fragebögen beschrieben.

\subsubsection{Stichprobe}

Die Online-Erhebung basiert auf einer Stichprobe von 384 Personen. Damit wurde eine Rücklaufquote von 13 Prozent erreicht. Die erfassten Hintergrundvariablen der Befragten geben Auskunft über ihre soziodemografische Verortung (siehe Tabelle 1). 
Tabelle 1. Stichprobe der Online-Befragung - Personenebene

\begin{tabular}{lll}
\hline & Pädagogische Fachkraft & Lehrkraft \\
\hline Anzahl $(\mathrm{n}=376)^{13}$ & $306(81 \%)$ & $70(19 \%)$ \\
\hline Geschlecht & $280(93 \%)$ weiblich & $63(91 \%)$ weiblich \\
$(\mathrm{n}=370)$ & $21(7 \%)$ männlich & $6(9 \%)$ männlich \\
\hline Regionale Verteilung & alle 16 Bundesländer vertreten, > $50 \%$ aus Nordrhein- \\
& Westfalen, Bayern und Baden-Württemberg \\
\hline MINT-Hintergrund & ja: $79(26 \%)$ & ja: $52(74 \%)$ \\
(n=372) & nein: $223(74 \%)$ & nein: $18(26 \%)$ \\
\hline Berufserfahrung in Jahren (MW) & $19,6(S D 11,9 ; n=296)$ & $16,7(S D 9,2 ; n=64)$ \\
\hline Anzahl besuchter Fortbildungen im & $4,5(S D 3,5)$ & $3,6(S D 4,6)$ \\
„Haus der kleinen Forscher“ (MW) & & \\
\hline
\end{tabular}

Die Befragten aus den Grundschulen waren zu 93 Prozent Lehrkräfte und zu 7 Prozent pädagogische Fachkräfte. Bezüglich der regionalen Verteilung lässt sich feststellen, dass Groß-, Mittel- und Kleinstädte sowie ländlich gelegene Ortschaften annähernd gleichermaßen in der Stichprobe vertreten waren. Bei der Berufserfahrung zeigt sich, dass im Durchschnitt die pädagogischen Fachkräfte länger ihren Beruf ausübten und auch mehr Fortbildungen der Initiative „Haus der kleinen Forscher" besucht hatten. In Tabelle 2 ist aufgelistet, in welchen Einrichtungsformen die Befragten arbeiteten und ob diese durch die Stiftung „Haus der kleinen Forscher" zertifiziert waren. Entsprechend der Stichprobenverteilung arbeitete der Großteil der Stichprobe in Kindertagesstätten, gefolgt von Grundschulen und schlussendlich den Horten.

Tabelle 2. Stichprobe der Online-Befragung - Einrichtungsebene

\begin{tabular}{llll}
\hline & Kitas & Horte & Grundschulen \\
\hline $\begin{array}{l}\text { Einrichtungen } \\
(\mathrm{n}=374)\end{array}$ & $276(74 \%)$ & $30(8 \%)$ & $68(18 \%)$ \\
\hline $\begin{array}{l}\text { Zertifizierung } \\
(\mathrm{n}=374)\end{array}$ & $\begin{array}{l}96 \text { von } 276 \\
(35 \%)\end{array}$ & $\begin{array}{l}8 \text { von } 30 \\
(27 \%)\end{array}$ & $\begin{array}{l}10 \text { von } 68 \\
(16 \%)\end{array}$ \\
\hline
\end{tabular}

Sowohl in Hinblick auf das Geschlecht, die Anzahl der besuchten Fortbildungen sowie die Einrichtungstypen (Kita, Hort, Grundschule) kann davon ausgegangen werden, dass die Stichprobe die Klientel der Initiative „Haus der kleinen Forscher“ in etwa repräsentativ abbildet (vgl. Abschnitt B 2.3, Stiftung Haus der kleinen Forscher, 2015a, 2017). Hinsichtlich der Zertifizierungen unterscheidet sich die Stichprobe jedoch deutlich von der Grundgesamtheit der an den Stiftungsangeboten teilnehmenden Einrichtungen, von denen insgesamt nur 16 Prozent zerti-

13 Abweichungen in der Anzahl von der Gesamtstichprobe sind auf Missings zurückzuführen. 
fiziert sind. Dies könnte ein Hinweis darauf sein, dass es sich bei der Stichprobe möglicherweise um eine besonders motivierte Personengruppe handeln könnte, denn eine Bedingung für die Zertifizierung einer Einrichtung ist ein erhöhtes Engagement im MINT-Bereich.

\subsubsection{Auswertung der Fragebögen}

Eine erste quantitativ-deskriptive Auswertung des Online-Fragebogens erfolgte automatisch im genutzten Programm EvaSyS, sodass die zentralen Tendenzen im Antwortverhalten sichtbar wurden.

Um Aussagen zur Hauptfragestellung, nämlich über Entwicklungsverläufe, treffen zu können, musste in der vorliegenden Studie auf Hilfskonstruktionen zurückgegriffen werden, da aus projektorganisatorischen Gründen keine Längsschnittstudie möglich war. Dazu wurden Daten von mehr und weniger erfahrenen Fach- und Lehrkräften miteinander verglichen in der Annahme, dass weniger erfahrene am Anfang stehen und erfahrene weiter fortgeschritten in ihrem Entwicklungsverlauf sind. Die Erfahrung wurde im Fragebogen durch drei Items abgebildet (siehe auch Tabelle 1), nämlich der MINT-Hintergrund, die Berufserfahrung und die Anzahl besuchter MINT-Fortbildungen der Initiative „Haus der kleinen Forscher“. Um dann Unterschiede zwischen erfahrenen und weniger erfahrenen Fach- und Lehrkräften zu ermitteln, wurden T-Tests berechnet, die für jeweils zwei unabhängige Stichproben überprüfen, ob sich deren Mittelwerte in Bezug auf festgelegte Zielvariablen unterscheiden, sowie einfaktorielle Varianzanalysen bei mehr als zwei unabhängigen Stichproben. ${ }^{14}$ Als Gruppierungsvariablen wurden erstens die Berufserfahrung, zweitens der MINT-Hintergrund, drittens die Anzahl der besuchten Fortbildungen und viertens die Unterscheidung nach Fach- und Lehrkraft herangezogen. Zur Analyse der Berufserfahrung wurde die Stichprobe in drei Gruppen aufgeteilt, welche eine Unterscheidung nach Anfängerinnen und Anfängern, Fortgeschrittenen und Profis möglich macht. Berufsanfängerinnen und -anfänger besitzen nach der vorliegenden Einteilung null bis zwei Jahre Erfahrung (8 Personen), Fortgeschrittene drei bis zehn Jahre (87 Personen) und Profis sind über zehn Jahre (254 Personen) in ihrem Berufsfeld tätig. Diese Einteilung wurde vorgenommen, um sowohl die erste Zeit nach der Berufsausbildung wie auch die Profis mit mehr als einem Jahrzehnt Berufserfahrung voneinander abzugrenzen. Aufgrund der geringen Fallzahl in der ersten Gruppe der Berufsanfängerinnen und

14 Hier wurde jeweils die Varianzgleichheit der Gruppen mithilfe des F- oder auch Levene-Tests überprüft, um dann die geeigneten Post-hoc-Analysen auszuwählen und die signifikanten Ergebnisse auf Gruppenunterschiede zu untersuchen. Bei gleichen Varianzen und stark unterschiedlicher Fallzahl wurde der GT2 nach Hochberg genutzt, bei ungleichen Varianzen (gleiche oder ungleiche Fallzahl) der Games-Howell. 
Berufsanfänger wurde zum Vergleich eine neue Gruppierung gebildet: Gruppe 1 mit null bis fünf Jahren Berufserfahrung (33 Personen), Gruppe 2 mit sechs bis zehn Jahren Berufserfahrung (62 Personen) und Gruppe 3 mit mehr als zehn Jahren Erfahrung (254 Personen). Die Varianzanalyse mit der neuen Dreiergruppierung kommt jedoch zu denselben Ergebnissen wie die erste Einteilung, sodass diese weiterführend berichtet wird.

Zur Unterscheidung zwischen mehr oder weniger MINT-fortbildungserfahrenen Fach- und Lehrkräften in Hinblick auf die Anzahl der besuchten Fortbildungen wurde folgende Aufteilung vorgenommen: Gruppe 1 mit einer beziehungsweise zwei Fortbildungsbesuchen, Gruppe 2 mit drei bis sechs Fortbildungsbesuchen, Gruppe 3 mit sieben bis neun Fortbildungsbesuchen und Gruppe 4 mit mehr als neun Fortbildungen. Diese Gruppeneinteilung wurde in Anlehnung an den Monitoring-Bericht der Stiftung „Haus der kleine Forscher“ (2016/2017) für die Studie weiterentwickelt (Stiftung Haus der kleinen Forscher, 2017).

Getestet wurden die Unterschiede zwischen den oben beschriebenen Gruppen hinsichtlich verschiedener Facetten des Aspekts Professionswissen: der „Forschungskreis“ (vgl. Marquardt-Mau, 2004, 2011; Rank et al., 2018; Stiftung Haus der kleinen Forscher, 2013) als Teil des fachdidaktischen Wissens und weitere Items. Beim Aspekt Motivation wurden die Unterschiede in Hinblick auf die Selbstwirksamkeitserwartung sowie die Anzahl der wiederholten Beispielexperimente und die Anzahl bereits umgesetzter Forschungsaktivitäten überprüft. Unterschiede zum Aspekt der Einstellungen wurden anhand verschiedener Fragen zu den Einstellungen zu MINT-Themen identifiziert.

Um nicht nur auf Einzelitemebene die Entwicklungsverläufe der Fach- und Lehrkräfte identifizieren zu können, wurden Indizes zu den drei Kompetenzfacetten gebildet. Hier wäre die Berechnung über eine explorative Faktorenanalyse möglich gewesen, es wurde jedoch folgendes Vorgehen gewählt: In einem argumentativen Validierungsprozess (Döring \& Bortz, 2016) wurden in mehreren Diskussionsrunden durch Expertinnen und Experten die Zusammensetzung der Indizes diskutiert.

Der Index „Professionswissen“ ergibt sich aus der Summe aller mindestens ordinalen Items, die die selbsteingeschätzten Facetten des Professionswissens der Befragten ermitteln (Item 4.1 bis $4.6+6.1+6.3+6.5+6.6+6.8$ bis $6.17+$ $6.19+8.3+8.6+8.9+8.12+8.15+8.18+8.21)$. Hierbei konnten Werte zwischen 28 (= sehr geringes selbst berichtetes Professionswissen) und 154 (= sehr hoch selbsteingeschätztes Professionswissen) erzielt werden. Der entsprechende Cronbachs-Alpha-Wert liegt bei $\alpha=0,88$ und belegt somit eine hohe Reliabilität der Skala.

Zur Bestimmung von Motivationsverläufen wurde auch hier ein Index gebildet. Der Index „Motivation“ ergibt sich aus der Summe von zwölf Items (Item 2.3 
+3.1 bis $3.6+6.4+7.1+7.4+8.22$ (nur Häufigkeit) +8.23$)$. Es konnten Werte zwischen 12 (= sehr geringe Motivation) und 61 (= sehr hohe Motivation) erreicht werden. Der entsprechende Cronbachs-Alpha-Wert liegt bei $\alpha=0,68$ und belegt somit eine akzeptable Reliabilität der Skala.

Korrespondierend zum Professionswissen und zur Motivation wurde auch zur Erfassung von Einstellungen ein kumulierter Index „Einstellungen“ errechnet. Dieser basiert auf der Summe dreier Items (Item $2.1+2.2+5.7) .{ }^{15}$ Die Befragten konnten Werte zwischen 3 (= sehr negative Einstellung) und 12 (= sehr positive Einstellung) erzielen. Der entsprechende Cronbachs-Alpha-Wert liegt allerdings nur bei $\alpha=0,44$, was auf eine geringe Reliabilität der Skala hindeutet, aber auch auf die niedrige Anzahl eingeflossener Items zurückgeführt werden kann. Aufgrund dessen sind entsprechende Ergebnisse in der Auswertung mit Vorsicht zu interpretieren.

Die finale Analyse des erhobenen Datenmaterials mündete in einer Zusammenführung zwischen den qualitativ und quantitativ gewonnenen Daten in Hinblick auf die Forschungsfragen der vorliegenden Studie, die im nächsten Kapitel dargestellt werden.

15 Die übrigen Items, die in Kapitel 6.3.3 den Einstellungen zugeordnet wurden, ließen sich aufgrund der fehlenden metrischen Antwortskala nicht im Index abbilden. 


\section{Ergebnisse der Studie}

In diesem Kapitel werden die Ergebnisse der Studie vorgestellt und in Hinblick auf die Forschungsfragen zusammengeführt. Hauptfragestellung der vorliegenden Studie ist es, Hinweise über die Entwicklungsverläufe von pädagogischen Fachund Lehrkräften in Bezug auf die Professionalisierung im MINT-Bereich zu generieren, da bisher dazu keine Forschungsergebnisse vorliegen.

\subsection{Stand der Professionalisierung im MINT-Bereich - Beantwortung der Unter-Forschungsfragen}

Die Beantwortung der Forschungsfragen FF U1 bis FF U4 stellt eine Annäherung dar, wo die pädagogischen Fach- und Lehrkräfte in Hinblick auf ihre Professionalisierung im MINT-Bereich stehen.

\subsubsection{FF U1 Wo stehen pädagogische Fach- und Lehrkräfte in Bezug auf forschendes Lernen mit Kindern nach ihrer eigenen Einschätzung?}

\section{In Bezug auf ihr Professionswissen}

Das selbst wahrgenommene Professionswissen der befragten Fach- und Lehrkräfte wurde in Bezug auf drei Aspekte erhoben: ihr Fach- und fachdidaktisches Wissen sowie ihre professionellen Verhaltenspotenziale. Das erhobene fachdidaktische Wissen bezieht sich zum einen auf die Kompetenzen in der Umsetzung des „Forschungskreises“ (vgl. Marquardt-Mau, 2004, 2011; Rank et al., 2018; Stiftung Haus der kleinen Forscher, 2013), ein Handwerkszeug zum strukturierten Forschen mit Kindern als Orientierungshilfe und Anleitung für die pädagogischen Fach- und Lehrkräfte. Zum anderen auf sechs ausgewählte Beispielsachverhalte, bei denen die Befragten einschätzen sollten, wie gut sie diese den Kindern erklären können. Das professionelle Verhaltenspotenzial wurde über die Nennung von Handlungsoptionen anhand zweier ausgewählter Beispielsituationen erhoben.

Die pädagogischen Fach- und Lehrkräfte aus den Interviews gaben an, dass sich durch die Fortbildungen der Initiative „Haus der kleinen Forscher“ ihr Hintergrundwissen zu MINT-Themen verbessert hat. Sie schätzten die konkreten Anregungen zum professionellen Handeln und zur Didaktik im Allgemeinen, die dort vermittelt wurden, und fühlten sich dadurch gestärkt, spontan bei Fragen der Kinder Forschungsaktivitäten durchzuführen. Die Selbstwahrnehmung des Professionswissens der Interviewten wurde nach eigener Angabe gefördert durch leicht anzuwendende Materialien (65 Nennungen) und verbessertes didaktisches Wis- 
sen zum Forschen mit Kindern (36 Nennungen), was die Befragten im Allgemeinen auf die Fortbildungen zurückführten (28 Nennungen) und dort in Zusammenhang gebracht wurde mit der Vermittlung von MINT-spezifischen Hintergrundinformationen (sieben Nennungen).

Im Folgenden werden beispielhaft Aussagen aus den Interviews zur Anwendung des „Forschungskreises“ (vgl. Marquardt-Mau, 2004, 2011; Rank et al., 2018; Stiftung Haus der kleinen Forscher, 2013) herangezogen. Eine Interviewteilnehmerin (pädagogische Fachkraft, Kita in Brandenburg) berichtete von ihren Schwierigkeiten in der Anwendung des „Forschungskreises“:

„Also mir fällt es schwer, diesen Forschungskreis umzusetzen, weil ich daran selber- also das macht man da irgendwie selber. Das geht ja da um Dokumentieren und welches Material man braucht, und wozu man das braucht und so weiter. Den Forschungskreis an sich habe ich eigentlich nie angewendet, außer in den Fortbildungen. Ja also das hat man irgendwie im Gefühl, also ich hab' da nie so die einzelnen Stationen so abgearbeitet. Das kommt irgendwie so von alleine, fand ich. Also wenn man das dann immer wieder in den Fortbildungen so hat, dann ist das so der Punkt, auf den ich mich nicht so freue, aber es gibt ja vielleicht auch Leute, die den auch aktiv nutzen. “

Eine andere Interviewpartnerin (pädagogische Fachkraft, Kita in Baden-Württemberg) formulierte dagegen ihre Fortschritte:

„Am Anfang haben wir uns mit dem Forschungskreis schwergetan. Aber jetzt, je mehr Fortbildungen wir besuchen, je mehr wir damit arbeiten, desto einfacher wird das. So langsam wird der Forschungskreis einsichtiger für uns. Eigentlich haben wir das auch schon immer so gemacht, nur halt nicht so richtig bewusst. Jetzt ist es uns richtig bewusst, dass wir den Kindern was anbieten. Hier, probier' das doch mal aus, die stellen neue Fragen und dann geht die Spirale halt weiter, dass die Kinder immer mehr lernen. “

Vor allem die Interviews machten deutlich, worin der eigentliche Nutzen der verschiedenen Fortbildungen für die befragten Fach- und Lehrkräfte besteht. Sie äußerten mehrheitlich, dass sie den Förderbereich MINT relevant für Kinder hielten und dass sie dort lernen würden, wie man ihn optimal an die Kinder heranführt.

Die Angaben der Teilnehmenden in der Online-Befragung zum forschenden Lernen mithilfe des „Forschungskreises“ (Items 8.1 bis 8.21; vgl. Marquardt-Mau, 2004, 2011; Rank et al., 2018; Stiftung Haus der kleinen Forscher, 2013) fielen mehrheitlich positiv aus (siehe Abbildung 8). Die Mittelwerte sind bei diesen Items besonders hoch: Ihre Kompetenzen, diese Methode und ihre Teilaspekte 
anwenden zu können, schätzten die Befragten durchschnittlich mit 8,3 von 10 möglichen Punkten ein. Dies deutet daraufhin, dass sie sich durchaus didaktischmethodisch in der Lage fühlen, mit Kindern nach dieser didaktischen Vorlage zu MINT-Themen zu forschen.

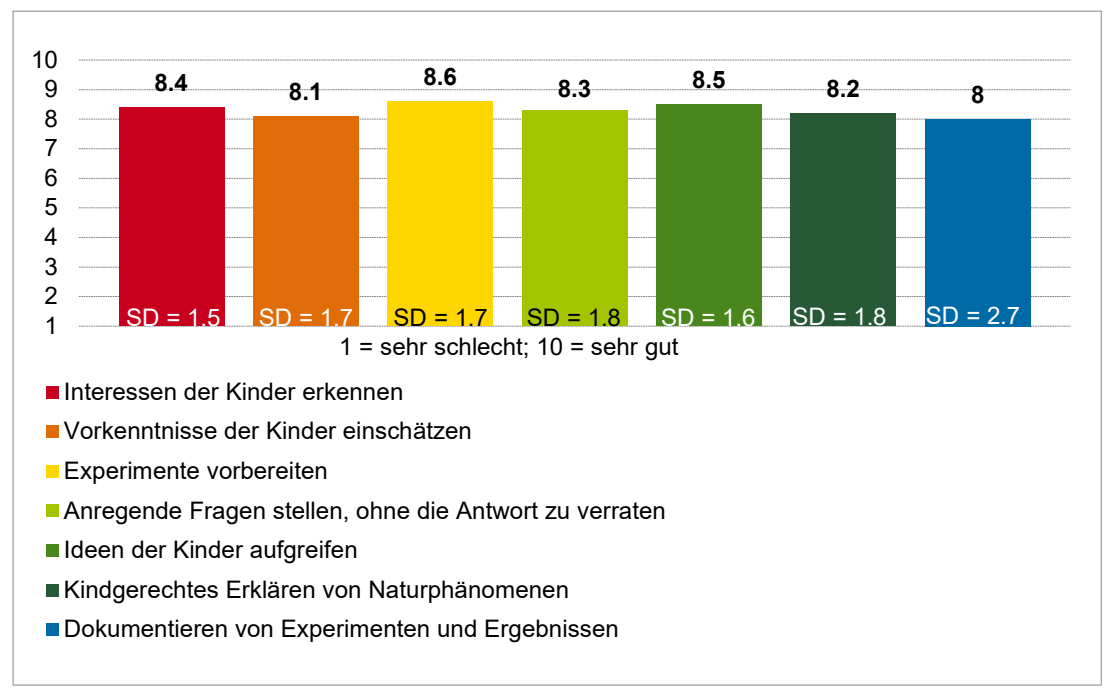

Abbildung 8. Selbstauskünfte zum Professionswissen (Items 8.3, 8.6, 8.9, 8.12, 8.15, 8.21)

In den Interviews fanden sich an verschiedenen Stellen Aussagen dazu, welche Aufgaben eine Fachkraft nach Ansicht der Befragten hat. Besonders häufig wurde das Erkennen von Gefahren und das Fördern selbstständigen Ausprobierens genannt, aber auch das Geben von Hilfestellungen und Anregungen. Des Weiteren thematisierten die Interviewten als Aufgabe, um Bildung zu ermöglichen, die Förderung von Kreativität, das Handelnd-erfahren-Lassen, das Erkennen von Interessen und die Orientierung daran sowie die Berücksichtigung des Alters der Kinder und ein angemessenes Eingehen auf ihre Fragen. Weniger häufig wurden die Förderung von Teamgeist und individuellen Stärken sowie das Besprechen von Forschungsergebnissen genannt (siehe Abbildung 9). 


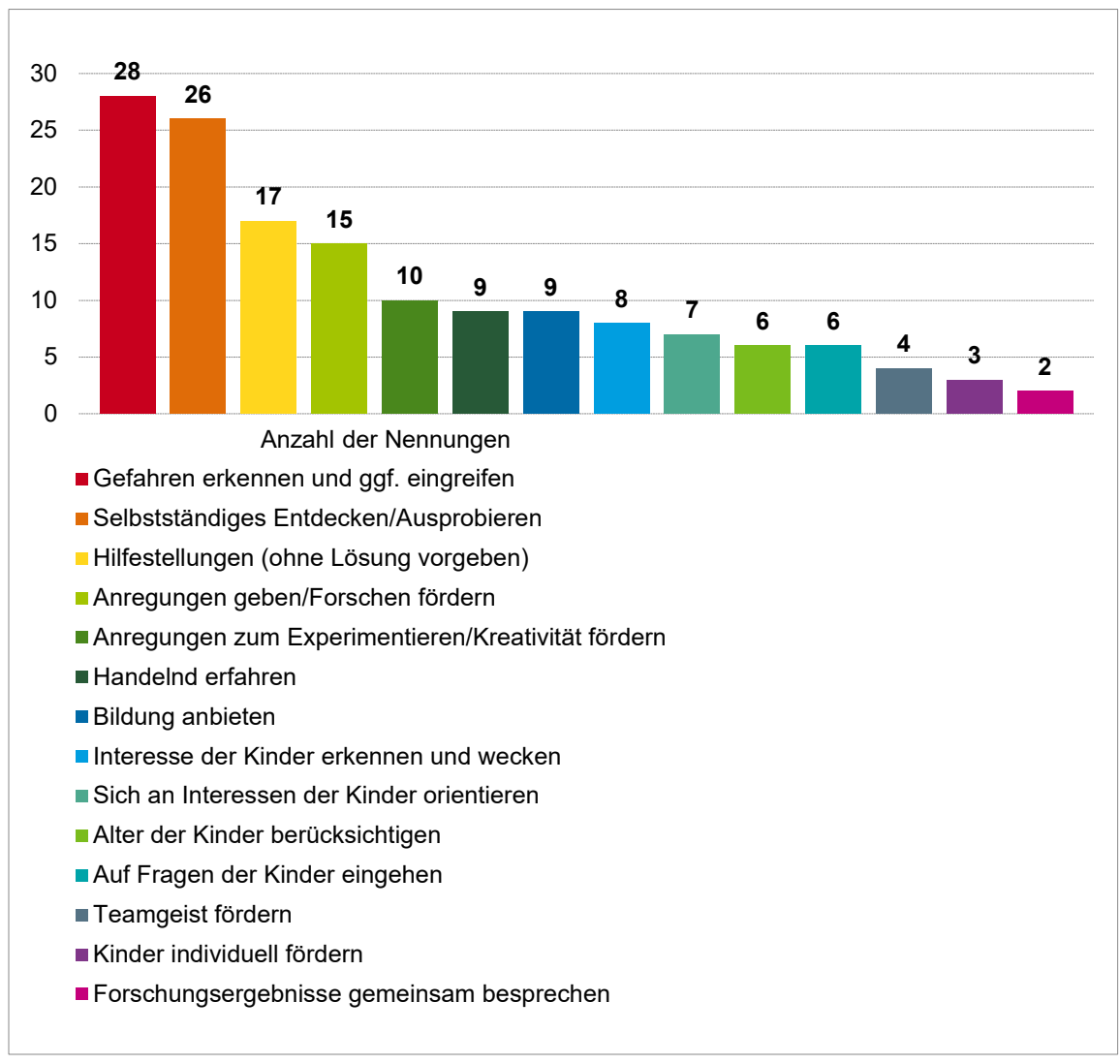

Abbildung 9. Nennungen zu Aufgaben einer guten pädagogischen Fachkraft (Interviewbefragung)

Zur Erfassung des potenziellen professionellen Verhaltens der Befragten wurden für den Online-Fragebogen zwei Beispielssituationen beschrieben. Zu der ersten Beispielsituation wurden elf aus den Interviews abgeleitete Verhaltensoptionen als Mehrfachwahlaufgabe angeboten (Item 4.7); fünf davon sollten ausgewählt werden. Zu der zweiten Beispielsituation wurden Verhaltensoptionen angeboten, zu denen die Zustimmung der Pädagoginnen und Pädagogen erfragt wurde (Item 4.8 bis 4.16). Bei den Situationen, in denen Kinder beim Forschen unterstützt werden sollen, waren die am häufigsten gewählten und somit als am wichtigsten eingeschätzten Verhaltensoptionen folgende: die Kinder zunächst selbstständig Dinge ausprobieren lassen, beobachten und die Beobachtungen gemeinsam besprechen, bei Misserfolgen Anregungen geben, dabei jüngere und ältere Kinder stets gleichermaßen miteinbeziehen und nur bei Gefahr eingreifen.

Diese Verhaltensoptionen gehen wiederum größtenteils konform mit dem „Forschungskreis“ (vgl. Marquardt-Mau, 2004, 2011; Rank et al., 2018; Stiftung 
Haus der kleinen Forscher, 2013). Differenzen ergaben sich dahingehend, dass nicht häufiger angegeben wurde, vor einer Forschungsaktivität Ideen und Vermutungen der Kinder zu sammeln sowie zum Schluss die Ergebnisse zu dokumentieren und zu erörtern.

70 Prozent der Online-Befragten berichteten, dass sie bei ihrer letzten Forschungsaktivität die Kinder überwiegend eigenständig Dinge ausprobieren und forschen ließen (Item 9.2). Unterstützt wurden die Kinder vor allem durch das Eingehen auf ihre selbst entwickelten Ideen und bei der Benutzung der zur Verfügung gestellten Instrumente und Materialien (Item 9.3). Besonderer Bedarf, die Kinder zu unterstützen, ergab sich beim Dokumentieren der Forschungsergebnisse (Item 9.4). Zum Abschluss der Forschungsaktivitäten wurden mehrheitlich die Beobachtungen der Kinder, ihr Vorgehen beim Ausprobieren und die Ergebnisse in Beziehung zu den Vermutungen der Kinder zu Beginn besprochen (Item 9.6).

Ihr fachdidaktisches Wissen zu den Beispielsachverhalten, die sich auf ausgewählte Bereiche der verschiedenen Fortbildungen zu MINT-Themen beziehen (im Fragebogen Item 4.1 bis 4.6), schätzten die Fach- und Lehrkräfte durchschnittlich als gut ein (mit 3 von 4 Punkten). Über 80 Prozent der Befragten gaben an, dass sie Kindern erklären könnten, warum etwa Holz auf dem Wasser schwimmt, aber ein Stein untergeht; warum unterschiedlich schwere Kinder zusammen wippen können, wenn das schwerere Kind weiter innen sitzt, warum nur ganz bestimmte Materialien von Magneten angezogen werden und wie der menschliche Körper funktioniert. Etwas geringer, nämlich zu 78 Prozent, wurde angegeben, dass sich die Befragten in der Lage fühlten, die Funktionsweise von Ampeln Kindern näher bringen zu können, und 72 Prozent trauten sich zu, Kindern zu erklären, warum es keine größte und keine kleinste Zahl gibt (siehe Abbildung 10).

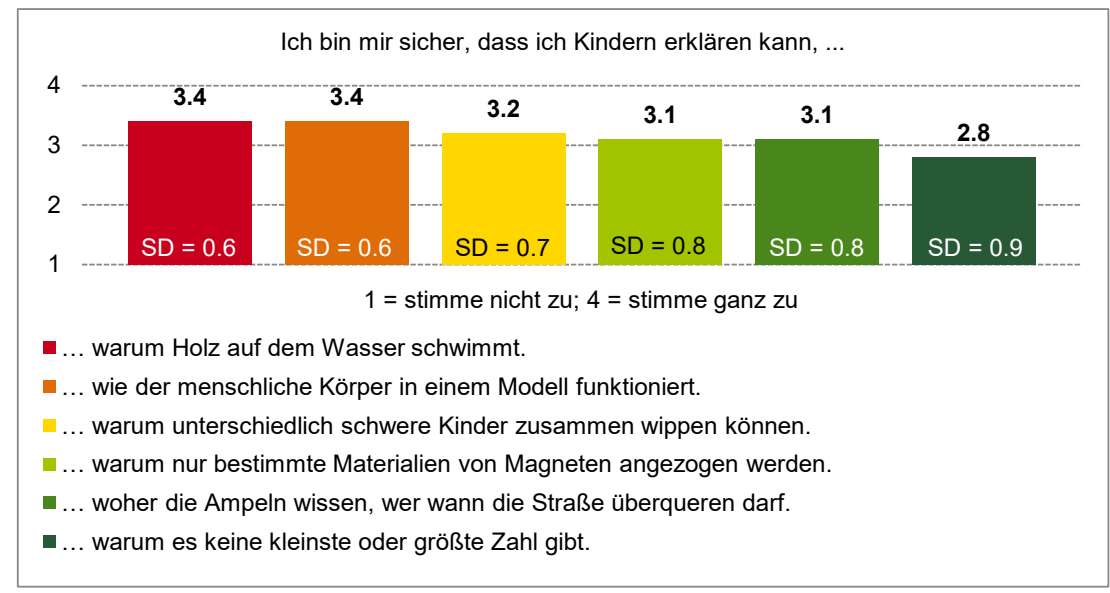

Abbildung 10. Selbstauskünfte über das fachdidaktische Wissen (Item 4.1-4.6) 


\section{In Bezug auf ihre Motivation, mit Kindern zu forschen}

Nach Aussage der Interviewteilnehmerinnen und -teilnehmer steigerten die Fortbildungen der Initiative „Haus der kleinen Forscher“ ihre Motivation, sich mit MINT-Themen vermehrt sowohl in der eigenen Einrichtung (zehn Nennungen) als auch privat (vier Nennungen) zu beschäftigen. Motivierend würden vor allem die Anregungen zum Forschen mit Kindern (38 Nennungen) wirken, die Beschäftigung mit MINT-Themen im Allgemeinen (14 Nennungen) und die Anwendbarkeit der zur Verfügung gestellten Materialien (13 Nennungen).

Als relevantes Motivationsmerkmal hinsichtlich des Forschens mit Kindern wurde in der Online-Befragung die Selbstwirksamkeitserwartung (Item 3.1-3.6) der Befragten erhoben (siehe Abschnitt A 6.3.3). Diese gibt an, wie hoch die Erwartung der Befragten ist, auf Grundlage ihrer eigenen Kompetenzen MINT-spezifische Sachverhalte an Kinder zu vermitteln. Erzielt werden konnten Punktwerte zwischen 6 und 24. Der errechnete Durchschnitt liegt bei 19,6, fällt also durchaus optimistisch aus.

In Item 2.3 wurde die Bereitschaft, sich auch in der eigenen Freizeit mit MINTThemen zu beschäftigen, abgefragt. Die Antwort unter den Befragten ist breit gestreut, 69 Prozent von ihnen stimmten einer solchen Bereitschaft eher oder voll zu.

\section{In Bezug auf ihre Einstellungen zu MINT-Themen}

Insgesamt zeigte sich, dass sich die Interviewaussagen zu Einstellungen primär auf das Forschen mit Kindern bezogen (208 Nennungen). Dennoch wurden auch Statements zu MINT-Themen im Allgemeinen (34 Nennungen) und den Fortbildungen im Speziellen (11 Nennungen) getroffen.

Die Aussagen zu den Subkategorien in der Hauptkategorie „Einstellungen“ verteilten sich in den Interviews folgendermaßen: Am häufigsten wurde genannt, dass den Kindern MINT-Themen im Alltag begegnen und diese deshalb wichtig sind (zehn Nennungen). Ähnlich oft wurde die Wichtigkeit des frühen MINT-Kontakts benannt (neun Nennungen). Wichtig schienen auch der Ausgleich des fehlenden MINT-Kontakts im Elternhaus (sechs Nennungen) durch die Einrichtung und die Ermöglichung eines Einblicks in verschiedene Themenbereiche im Laufe der Kita- und Schulzeit (vier Nennungen). Der globale Ansatz, dass MINT-Bildung Deutschland langfristig hilft, wurde dreimal benannt. Zweimal wurde einschränkend angemerkt, dass MINT-Bildung nur ein Aspekt von vielen sei, den Bildungseinrichtungen berücksichtigen sollten (siehe Abbildung 11). 


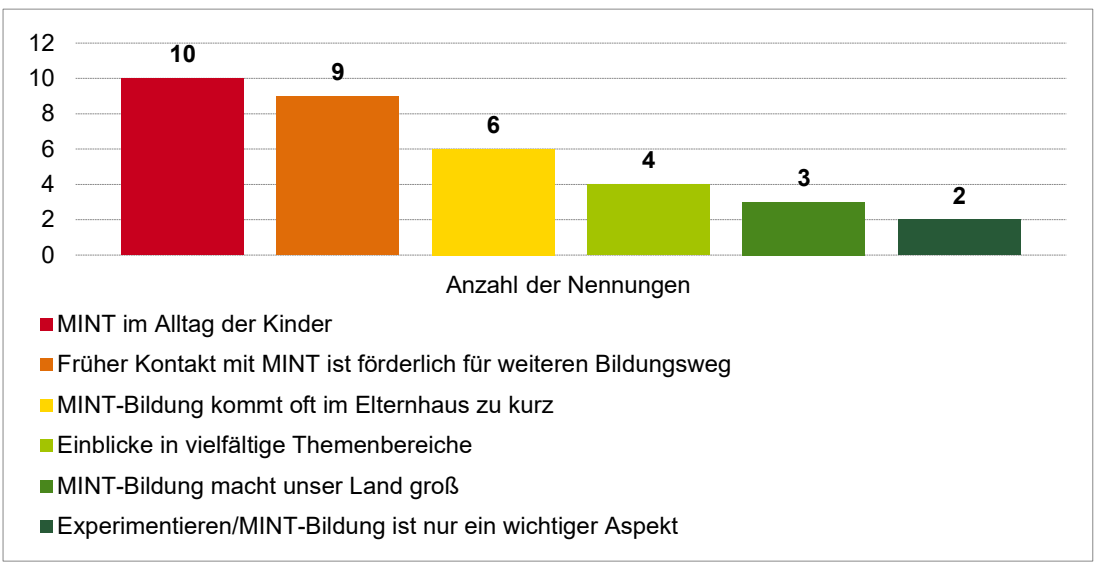

Abbildung 11. Einstellungen zu MINT-Themen (Interviewbefragung)

Einstellungen zum Forschen mit Kindern wurden überwiegend dort geäußert, wo es darum ging, inwiefern die Fortbildungen der Initiative „Haus der kleinen Forscher“ das Bild einer guten pädagogischen Fach- beziehungsweise Lehrkraft beeinflussen. Was den Interviewten besonders in Erinnerung geblieben ist, waren zum Teil Umsetzungsschwierigkeiten bei der Anwendung der Inhalte aus den Fortbildungen. Dabei bezogen sich die Aussagen auf Umsetzungsschwierigkeiten mit dem „Forschungskreis“ (vgl. Marquardt-Mau, 2004, 2011; Rank et al., 2018; Stiftung Haus der kleinen Forscher, 2013) sowie auf äußere Gegebenheiten (,Das Problem, das ich dabei sehe ist - gerade auch in unserer Schule gibt es ein akutes Platzproblem. So was will man dann ja auch stehen lassen, aber kann man nicht in einer Stunde behandeln. [...]“) oder Fähigkeiten der Kinder, (,Es müssen halt nur noch viele Kinder lernen, länger dabei zu bleiben und die muss man dann bei der Stange halten. ").

Auf die Frage, wieso sich bewusst für die Teilnahme an den Fortbildungen entschieden wurde, äußerten viele Interviewte, dass ihr Interesse an MINT und am Forschen im Allgemeinen häufig auch schon vor den besuchten Fortbildungen ausgeprägt gewesen war. Durch den Besuch einer Fortbildungsveranstaltung der Initiative „Haus der kleinen Forscher“ sei ihnen bewusst geworden, dass die Forschungsanregungen beziehungsweise „Experimente“ aus den Fortbildungen leicht umsetzbar seien. Einige Befragte äußerten zudem, die Fortbildung habe ihre Einstellungen dahingehend verändert, dass beim Forschen nicht immer alles funktionieren muss und sie nicht immer alle Zusammenhänge erklären können müssen, nicht zuletzt auch deshalb, weil die Kinder beim Experimentieren beziehungsweise Entdecken und Forschen über MINT-Themen hinaus etwas lernen.

Zwei Items $(2.1,2.2$.) des Online-Fragebogens sollen an dieser Stelle in die Erfassung der Einstellungen eingehen. Den Förderbereich MINT in Kindertages- 
stätten hielten 91 Prozent der Befragten für wichtig bis sehr wichtig. MINT-Bildung in der Grundschule hielten sogar 95 Prozent für wichtig bis sehr wichtig.

Im Online-Fragebogen wurde auch erhoben, was den Fach- und Lehrkräften beim Forschen besonders wichtig ist (Items 2.4-2.8). Hier bekamen alle Antwortoptionen bei einer geringen Streuung hohe Zustimmungswerte, besonders die beiden Items, dass Kinder ihre eigenen Ideen entwickeln und früh lernen, ihren eigenen Fragestellungen nachzugehen (siehe Abbildung 12).

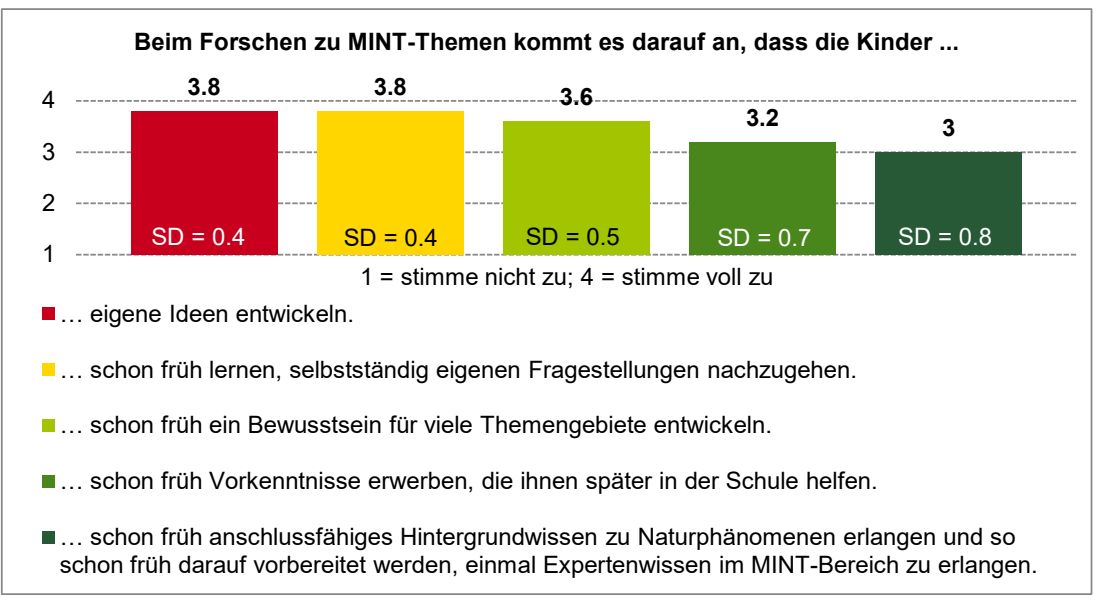

Abbildung 12. Lernziel/Zweck des Forschens mit Kindern (Item 2.4-2.8)

\section{Zusammenfassung FF U1}

Es zeigte sich, dass beim selbstberichteten Professionswissen die Prioritäten der Befragten konform mit dem Leitgedanken der Stiftung „Haus der kleinen Forscher" gehen, da sie angaben, großen Wert darauf zu legen, Kinder zunächst selbstständig Dinge ausprobieren zu lassen, zu beobachten und die Beobachtungen später zu besprechen. Entsprechend schätzten sich die Befragten des Online-Fragebogens selbst bezüglich der Umsetzung des „Forschungskreises“ (vgl. Marquardt-Mau, 2004, 2011; Rank et al., 2018; Stiftung Haus der kleinen Forscher, 2013) als kompetent bis sehr kompetent ein, in den Interviews gab es hierzu unterschiedliche Ansichten. Die Beispielsachverhalte (Online-Fragebogen Item 4.7-4.16) konnten die Befragten nach eigenen Angaben den Kindern gut erklären, wobei die meisten Schwierigkeiten darin gesehen wurden zu erklären, warum es keine größte und kleinste Zahl gibt. Auch hinsichtlich Motivation und Einstellungen zeichneten die Befragten von sich ein positives Bild: sie seien motiviert, mit Kindern zu MINT-Themen zu forschen und würden diese Themen auch für relevant halten.

Die Motivation, sich auch im Privaten mit MINT-Themen auseinanderzusetzen, war bei den Befragten hoch. Auch zeigte sich im Schnitt eine hohe Selbst- 
wirksamkeitserwartung der Befragten. MINT-Themen wurden als wichtig eingeschätzt, wobei die Relevanz von MINT-Themen in der Grundschule etwas höher eingeschätzt wurde als in Kindertagestätten.

Insgesamt lieferten sowohl die Ergebnisse der Interviews als auch der Fragebögen folglich ein positives Bild der MINT-bezogenen Kompetenzen der befragten Fach- und Lehrkräfte. Einschränkend muss jedoch bemerkt werden, dass es sich um selbsteingeschätzte Kompetenzen handelt, nicht um tatsächlich beobachtete.

\subsubsection{FF U2 Gibt es Unterschiede zwischen erfahrenen und weniger erfahrenen pädagogischen Fach- und Lehrkräften?}

Die Erfahrung der Fach- und Lehrkräfte wurde zur Beantwortung dieser Frage unter drei verschiedenen Bezugspunkten beleuchtet: die Berufserfahrung, der MINTHintergrund und die Anzahl der besuchten Fortbildungen bei der Initiative „Haus der kleinen Forscher" durch die Befragten. Dabei wurden die jeweiligen Unterschiede bezüglich des Professionswissens, der Motivation und der Einstellungen zu MINT untersucht.

\section{In Bezug auf das Professionswissen}

\section{Berufserfahrung}

Tabelle 3 zeigt, dass sich bezüglich der selbsteingeschätzten Kompetenzen, den „Forschungskreis“ (vgl. Marquardt-Mau, 2004, 2011; Rank et al., 2018; Stiftung Haus der kleinen Forscher, 2013) umsetzen zu können, keine signifikanten Unterschiede zwischen Anfängerinnen und Anfängern, Fortgeschrittenen und Profis erkennen lassen.

Tabelle 3. Einfluss der Berufserfahrung auf die Kompetenzen, den „Forschungskreis“ umsetzen zu können (Items 8.3, 8.6, 8.9, 8.12, 8.15, 8.18, 8.21)

\begin{tabular}{lccccc}
\hline Berufserfahrung in Jahren & $\begin{array}{c}0-2 \\
(\mathrm{n}=7)\end{array}$ & $\begin{array}{c}3-10 \\
(\mathrm{n}=84)\end{array}$ & $\begin{array}{c}>10 \\
(\mathrm{n}=249)\end{array}$ & $\mathrm{F}$ & Sig. \\
\hline Interessen der Kinder erkennen & 8.0 & 8.4 & 8.4 & 0.4 & .400 \\
\hline $\begin{array}{l}\text { Vorkenntnisse der Kinder } \\
\text { einschätzen }\end{array}$ & 8.0 & 8.1 & 8.1 & 0.1 & .585 \\
\hline Experimente vorbereiten & 8.1 & 8.4 & 8.6 & 1.2 & .083 \\
\hline Anregende Fragen stellen & 7.3 & 8.3 & 8.3 & 2.0 & .392 \\
\hline Ideen der Kinder aufgreifen & 8.1 & 8.5 & 8.5 & 0.4 & .459 \\
\hline Kindgerechtes Erklären & 7.7 & 8.1 & 8.2 & 0.5 & .963 \\
\hline Dokumentieren & 8.2 & 7.9 & 7.9 & 0.1 & .980 \\
\hline
\end{tabular}

einfaktorielle Varianzanalyse, * $p<.05$, zweiseitig 
Bezüglich der Einschätzung, wie gut spezifische Sachverhalte den Kindern erklärt werden können, zeigt Tabelle 4 allein beim Erklären des menschlichen Körpers anhand eines Modells signifikante Unterschiede. Diese lassen sich durch Post-hocAnalysen (Hochberg) als signifikante Unterschiede zwischen den Anfängerinnen und Anfängern sowie den Profis erklären (.025). Aber auch hinsichtlich der anderen Themenbereiche (mit Ausnahme der Ampelphasen) ist ein kleiner Anstieg des berichteten fachdidaktischen Wissens mit der Berufserfahrung zu erkennen, der aber nicht signifikant ist.

Tabelle 4. Einfluss der Berufserfahrung auf das fachdidaktische Wissen (Items 4.1-4.6)

\begin{tabular}{|c|c|c|c|c|c|}
\hline Berufserfahrung in Jahren & $\begin{array}{c}0-2 \\
(n=9)\end{array}$ & $\begin{array}{c}3-10 \\
(n=92)\end{array}$ & $\begin{array}{c}>10 \\
(n=282)\end{array}$ & $\mathrm{F}$ & Sig. \\
\hline \multicolumn{6}{|l|}{$\begin{array}{l}\text { Ich bin mir sicher, dass ich } \\
\text { Kindern erklären kann, ... }\end{array}$} \\
\hline $\begin{array}{l}\text {... warum Holz auf dem Wasser } \\
\text { schwimmt. }\end{array}$ & 3.1 & 3.4 & 3.5 & 2.2 & .108 \\
\hline $\begin{array}{l}\text {... warum unterschiedlich schwe- } \\
\text { re Kinder zusammen wippen } \\
\text { können. }\end{array}$ & 3.0 & 3.1 & 3.2 & 1.5 & .231 \\
\hline $\begin{array}{l}\ldots \text { warum nur bestimmte Mate- } \\
\text { rialien von Magneten angezogen } \\
\text { werden. }\end{array}$ & 2.8 & 3.0 & 3.1 & 1.8 & .160 \\
\hline $\begin{array}{l}\text {... wie der menschliche Körper in } \\
\text { einem Modell funktioniert. }\end{array}$ & 2.9 & 3.3 & 3.4 & $4.4^{\star}$ & .013 \\
\hline $\begin{array}{l}\text {... warum es keine kleinste oder } \\
\text { größte Zahl gibt. }\end{array}$ & 2.7 & 2.8 & 2.8 & 0.2 & .832 \\
\hline $\begin{array}{l}\text {... woher die Ampeln wissen, } \\
\text { wer wann die Straße überqueren } \\
\text { darf. }\end{array}$ & 3.3 & 3.1 & 3.1 & 0.6 & .563 \\
\hline
\end{tabular}

einfaktorielle Varianzanalyse, ${ }^{*} p<.05$, zweiseitig

Zieht man also die Berufserfahrung, operationalisiert über die Jahre im Beruf, als Gruppierungsvariable heran, zeigen sich hinsichtlich des selbsteingeschätzten Professionswissens keine bedeutsamen Unterschiede zwischen den Gruppen. Das bedeutet, erfahrene wie weniger erfahrene Fach- und Lehrkräfte schätzten ihr Professionswissen als vergleichbar hoch ein. 


\section{MINT-Hintergrund}

Fach- und Lehrkräfte können auch anhand ihres MINT-Hintergrunds unterschieden werden. Die Stichprobe wurde daher unterteilt in Fach- und Lehrkräfte, die unabhängig von der Initiative „Haus der kleinen Forscher“ bereits in einem MINTBereich Erfahrungen gesammelt haben, z. B. durch ein Studium, eine Ausbildung, als Teil der pädagogischen Ausbildung oder durch Weiterbildungen, und Fachund Lehrkräfte ohne besondere MINT-Erfahrungen. Tabelle 5 zeigt, dass sich beide Gruppen für gleich kompetent hielten, den „Forschungskreis“ (vgl. MarquardtMau, 2004, 2011; Rank et al., 2018; Stiftung Haus der kleinen Forscher, 2013) umzusetzen, also z. B. MINT-Themen kindgerecht zu erklären sowie Experimente und Ergebnisse zu dokumentieren.

Tabelle 5. Unterschied zwischen dem Vorhandensein eines MINT-Hintergrunds auf die Kompetenz, den „Forschungskreis“ umsetzen zu können (Items 8.3, 8.6, 8.9, 8.12, 8.15, $8.18,8.21)$

\begin{tabular}{lcccc}
\hline & $\begin{array}{c}\text { MINT- } \\
\text { Hintergrund } \\
(\mathrm{n}=120-124)\end{array}$ & $\begin{array}{c}\text { kein MINT- } \\
\text { Hintergrund } \\
(\mathrm{n}=204-211)\end{array}$ & $\mathrm{t}$ & Sig. \\
\hline Interessen der Kinder erkennen & 8.4 & 8.4 & -0.1 & .899 \\
\hline Vorkenntnisse der Kinder einschätzen & 8.1 & 8.1 & 0.5 & .617 \\
\hline Experimente vorbereiten & 8.7 & 8.5 & 1.1 & .253 \\
\hline Anregende Fragen stellen & 8.4 & 8.2 & 0.7 & .462 \\
\hline Ideen der Kinder aufgreifen & 8.5 & 8.5 & 0.0 & .982 \\
\hline Kindgerechtes Erklären & 8.3 & 8.1 & 1.4 & .157 \\
\hline Dokumentieren & 8.1 & 7.8 & 1.6 & .177 \\
\hline t-Test, * ${ }^{*}<05$ & & & &
\end{tabular}

Geht es konkret darum, spezifische Sachverhalte kindgerecht erklären zu können, schätzten sich die beiden Gruppen dagegen unterschiedlich ein (Tabelle 6): Im Vergleich gaben die Befragten mit einem MINT-Hintergrund ihre Fähigkeit, Kindern die Beispielsachverhalte zu erklären, als höher an als die Befragten ohne MINT-Hintergrund. 
Tabelle 6. Unterschied zwischen dem Vorhandensein eines MINT-Hintergrunds auf das fachdidaktische Wissen (Items 4.1-4.6)

\begin{tabular}{|c|c|c|c|c|}
\hline & $\begin{array}{c}\text { MINT- } \\
\text { Hintergrund } \\
(n=133-134)\end{array}$ & $\begin{array}{l}\text { Kein MINT- } \\
\text { Hintergrund } \\
(\mathrm{n}=241-244)\end{array}$ & $\mathrm{t}$ & Sig. \\
\hline \multicolumn{5}{|c|}{ Ich bin mir sicher, dass ich Kindern erklären kann, ... } \\
\hline $\begin{array}{l}\text {... warum Holz auf dem Wasser } \\
\text { schwimmt. }\end{array}$ & 3.5 & 3.4 & $2.7^{\star}$ & .007 \\
\hline $\begin{array}{l}\text {... warum unterschiedlich schwere Kinder } \\
\text { zusammen wippen können. }\end{array}$ & 3.3 & 3.1 & $2.9^{*}$ & .005 \\
\hline $\begin{array}{l}\text {... warum nur bestimmte Materialien von } \\
\text { Magneten angezogen werden. }\end{array}$ & 3.3 & 3.0 & $3.3^{*}$ & .001 \\
\hline $\begin{array}{l}\text {... wie der menschliche Körper in einem } \\
\text { Modell funktioniert. }\end{array}$ & 3.6 & 3.3 & $4.0^{\star}$ & .000 \\
\hline $\begin{array}{l}\text {... warum es keine kleinste oder größte } \\
\text { Zahl gibt. }\end{array}$ & 2.9 & 2.7 & $3.0^{\star}$ & .003 \\
\hline $\begin{array}{l}\text {... woher die Ampeln wissen, wer wann } \\
\text { die Straße überqueren darf. }\end{array}$ & 3.1 & 3.0 & 0.8 & .441 \\
\hline
\end{tabular}

t-Test, ${ }^{\star} \mathrm{p}<.05$

\section{Anzahl der besuchten Fortbildungen}

Unterteilt man die Gruppen in Personen mit ein bis zwei Fortbildungsteilnahmen, drei bis sechs Fortbildungsteilnahmen, sieben bis neun Fortbildungsteilnahmen und mehr als neun Fortbildungsteilnahmen, lassen sich Varianzanalysen heranziehen, die folgendes Ergebnis aufweisen.

Für die selbsteingeschätzte Kompetenz, den „Forschungskreis“ (vgl. Marquardt-Mau, 2004, 2011; Rank et al., 2018; Stiftung Haus der kleinen Forscher, 2013) anwenden zu können, zeigen sich signifikante Unterschiede in Bezug auf die Anzahl der Fortbildungsbesuche. Die Mittelwerte geben einen Hinweis darauf, dass Personen mit mehr besuchten Fortbildungen ihre Kompetenz in der Anwendung des „Forschungskreises“ (vgl. Marquardt-Mau, 2004, 2011; Rank et al., 2018; Stiftung Haus der kleinen Forscher, 2013) jeweils höher einschätzten (Tabelle 7). Signifikante Gruppenunterschiede finden sich in den Post-hoc-Analysen (Hochberg) zwischen den Personen, die ein bis zwei Fortbildungen besucht haben und denen, die bereits mehr als neun Fortbildungsbesuche aufweisen z. B. beim Item „Kindgerechtes Erklären .021 oder bei Item Vorkenntnisse der Kinder einschätzen .009). 
Tabelle 7. Einfluss der Fortbildungsanzahl auf die Kompetenzen, den „Forschungskreis“ umsetzen zu können (Items 8.1-8.21)

\begin{tabular}{lcccccc}
\hline $\begin{array}{l}\text { Anzahl besuchter } \\
\text { Fortbildungen }\end{array}$ & $\begin{array}{c}1-2 \\
(\mathrm{n}=103-110)\end{array}$ & $\begin{array}{c}3-6 \\
(\mathrm{n}=132-136)\end{array}$ & $\begin{array}{c}7-9 \\
(\mathrm{n}=46-47)\end{array}$ & $\begin{array}{c}\text { >9 } \\
(\mathrm{n}=46-48)\end{array}$ & $\mathrm{F}$ & Sig. \\
\hline $\begin{array}{l}\text { Interessen der } \\
\text { Kinder erkennen }\end{array}$ & 8.1 & 8.3 & 8.7 & 8.9 & $5.4^{*}$ & .001 \\
\hline $\begin{array}{l}\text { Vorkenntnisse } \\
\text { der Kinder ein- } \\
\text { schätzen }\end{array}$ & 7.9 & 8.1 & 8.2 & 8.6 & $3.6^{*}$ & .014 \\
\hline $\begin{array}{l}\text { Experimente vor- } \\
\text { bereiten }\end{array}$ & 8.3 & 8.6 & 8.9 & 8.9 & $3.9^{*}$ & .009 \\
\hline $\begin{array}{l}\text { Anregende Fra- } \\
\text { gen stellen }\end{array}$ & 8.0 & 8.3 & 8.5 & 8.9 & $5.5^{*}$ & .001 \\
\hline $\begin{array}{l}\text { Ideen der Kinder } \\
\text { aufgreifen }\end{array}$ & 8.3 & 8.4 & 8.9 & 8.8 & $3.9^{*}$ & .010 \\
\hline $\begin{array}{l}\text { Kindgerechtes } \\
\text { Erklären }\end{array}$ & 7.9 & 8.2 & 8.1 & 8.6 & $3.6^{*}$ & .014 \\
\hline $\begin{array}{l}\text { Dokumentieren } \\
\text { einfaktorielle Varianzanalyse, * } \mathrm{p}<.05, \mathrm{zw} \text { - }\end{array}$ & 7.6 & 8.8 & 8.4 & 8.4 & $3.8^{*}$ & .010 \\
\hline
\end{tabular}

Bezüglich der Einschätzung, spezifische Sachverhalte kindgerecht erklären zu können, fallen die Ergebnisse differenzierter aus: Lediglich die Themen „Hebelwirkung“ und „Magnetismus“ trauten sich Befragte mit steigender Anzahl an besuchten Fortbildungen eher zu, erklären zu können (siehe Tabelle 8). Beim Thema „Hebelwirkung“ besteht ein knapp nicht signifikanter Gruppenunterschied laut Post-hoc-Analysen (Hochberg) zwischen den Gruppen mit bisher ein bis zwei und den mit sieben bis neun besuchten Fortbildungen (Item 4.2 (Hebel): .057). ${ }^{16}$

In Bezug auf die Frage nach der Erklärung zu den Magneten (Item 4.3) zeigen die Post-hoc-Analysen einen signifikanten Unterschied (Hochberg) zwischen der Gruppe mit ein bis zwei Fortbildungen und derjenigen mit sieben bis neun (.011) beziehungsweise mit über neun Fortbildungen (.006).

16 Dies könnte auf eine Alpha-Fehler-Kumulierung hinweisen, die an dieser Stelle aber nicht weiter überprüft wurde. 
Tabelle 8. Einfluss der Fortbildungsanzahl auf das fachdidaktische Wissen (Items 4.1-4.6)

\begin{tabular}{|c|c|c|c|c|c|c|}
\hline $\begin{array}{l}\text { Anzahl besuchter } \\
\text { Fortbildungen }\end{array}$ & $\begin{array}{c}1-2 \\
(n=139-140)\end{array}$ & $\begin{array}{c}3-6 \\
(n=142-144)\end{array}$ & $\begin{array}{c}7-9 \\
(n=46-47)\end{array}$ & $\begin{array}{c}>9 \\
(n=50-53)\end{array}$ & $\mathrm{F}$ & Sig. \\
\hline \multicolumn{7}{|l|}{$\begin{array}{l}\text { Ich bin mir sicher, } \\
\text { dass ich Kindern er- } \\
\text { klären kann, ... }\end{array}$} \\
\hline $\begin{array}{l}\text {... warum Holz } \\
\text { auf dem Wasser } \\
\text { schwimmt. }\end{array}$ & 3.4 & 3.5 & 3.5 & 3.5 & 1.4 & .240 \\
\hline $\begin{array}{l}\text {... warum unterschied- } \\
\text { lich schwere Kinder } \\
\text { zusammen wippen } \\
\text { können. }\end{array}$ & 3.1 & 3.2 & 3.4 & 3.4 & $3.5^{\star}$ & .016 \\
\hline $\begin{array}{l}\text {... warum nur be- } \\
\text { stimmte Materialien } \\
\text { von Magneten an- } \\
\text { gezogen werden. }\end{array}$ & 2.9 & 3.1 & 3.3 & 3.3 & $5.5^{\star}$ & .001 \\
\hline $\begin{array}{l}\text {... wie der mensch- } \\
\text { liche Körper in einem } \\
\text { Modell funktioniert. }\end{array}$ & 3.3 & 3.4 & 3.5 & 3.5 & 1.9 & .126 \\
\hline $\begin{array}{l}\text {... warum es keine } \\
\text { kleinste oder größte } \\
\text { Zahl gibt. }\end{array}$ & 2.8 & 2.8 & 2.7 & 2.8 & 0.2 & .875 \\
\hline $\begin{array}{l}\text {... woher die Ampeln } \\
\text { wissen, wer wann die } \\
\text { Straße überqueren } \\
\text { darf. }\end{array}$ & 3.0 & 3.1 & 3.1 & 3.2 & 0.6 & .638 \\
\hline
\end{tabular}

einfaktorielle Varianzanalyse, ${ }^{\star} \mathrm{p}<.05$, zweiseitig

\section{In Bezug auf ihre Motivation}

\section{Berufserfahrung}

Die Ergebnisse zeigen signifikante Unterschiede zwischen den Gruppen der Berufsanfängerinnen und -anfänger, Fortgeschrittenen und Profis hinsichtlich der Selbstwirksamkeitserwartung, wobei die Befragten mit mehr als zehn Jahren Berufserfahrung den höchsten Mittelwert aufweisen. Zwischen Berufsanfängerinnen und -anfängern und Fortgeschrittenen wird ein signifikanter Unterschied verzeichnet (Tabelle 9), wie auch die Post-hoc-Analysen bestätigen (Bonferroni) (.006). Ein signifikanter Unterschied (.001) besteht zudem zwischen Berufsanfängerinnen und -anfängern und Profis (über 10 Jahre).

Keinen Unterschied gibt es hinsichtlich der Anzahl der wiederholten Beispielexperimente. Hinsichtlich der Anzahl bereits absolvierter Forschungsaktivitäten zeigen sich dagegen Unterschiede, die laut Post-hoc-Analysen (Bonferroni) vor allem zwischen den Anfängerinnen und Anfängern und den Fortgeschrittenen (.013) sowie den Anfängerinnen und Anfängern und den Profis (.006) bestehen. 
Tabelle 9. Einfluss der Berufserfahrung auf Selbstwirksamkeitserwartung, Anzahl wiederholter Beispielexperimente und Anzahl umgesetzter Forschungsaktivitäten (Items 3.1-3.6, 7.4 und 8.23)

\begin{tabular}{lccccc}
\hline Berufserfahrung in Jahren & $\begin{array}{c}0-2 \\
(\mathrm{n}=8)\end{array}$ & $\begin{array}{c}3-10 \\
(\mathrm{n}=87)\end{array}$ & $\begin{array}{c}>10 \\
(\mathrm{n}=254)\end{array}$ & $\mathrm{F}$ & Sig. \\
\hline Selbstwirksamkeitserwartung & 16.4 & 19.1 & 19.5 & $6.2^{*}$ & .002 \\
\hline Anzahl wiederholter Beispielexperimente & 2.1 & 2.5 & 2.6 & 2.0 & .141 \\
\hline Anzahl umgesetzter Forschungsaktivitäten & 2.5 & 4.4 & 4.5 & $5.0^{*}$ & .008 \\
\hline einfaktorielle Varianzanalyse, *p ${ }^{*} .05$, zweiseitig & & & &
\end{tabular}

\section{MINT-Hintergrund}

Pädagoginnen und Pädagogen mit MINT-Hintergrund zeigten eine signifikant höhere Selbstwirksamkeitserwartung als Pädagoginnen und Pädagogen ohne MINTHintergrund. Ebenso haben Personen mit MINT-Hintergrund signifikant mehr Forschungsaktivitäten umgesetzt. Hinsichtlich der Anzahl wiederholter Beispielexperimente, die bei den Fortbildungen der Initiative „Haus der kleinen Forscher“ durchgeführt wurden, kann jedoch kein signifikanter Unterschied zwischen Pädagoginnen und Pädagogen mit und ohne MINT-Hintergrund festgestellt werden (siehe Tabelle 10).

Tabelle 10. Unterschied zwischen dem Vorhandensein eines MINT-Hintergrunds auf die Selbstwirksamkeitserwartung, Anzahl wiederholter Beispielexperimente und Anzahl umgesetzter Forschungsaktivitäten (Items 3.1-3.6, 7.4 und 8.23)

\begin{tabular}{lcccc}
\hline & $\begin{array}{c}\text { MINT- } \\
\text { Hintergrund } \\
(\mathrm{n}=126-132)\end{array}$ & $\begin{array}{c}\text { Kein MINT- } \\
\text { Hintergrund } \\
(\mathrm{n}=234)\end{array}$ & $\mathrm{t}$ & Sig. \\
\hline Selbstwirksamkeitserwartung & 20.2 & 18.9 & $4.6^{*}$ & .000 \\
\hline Anzahl wiederholter Beispielexperimente & 2.5 & 2.6 & -0.6 & .542 \\
\hline Anzahl umgesetzter Forschungsaktivitäten & 5.1 & 4.1 & $4.9^{*}$ & .000 \\
\hline t-Test, ${ }^{*} p<.05$ & & & &
\end{tabular}

\section{Anzahl der besuchten Fortbildungen}

Eine Lehrkraft aus dem Saarland, die bereits sechs Fortbildungen besucht hatte, brachte im Interview ihre subjektiv wahrgenommenen Veränderungen in der Motivation und Selbstwirksamkeit auf den Punkt, was sich in der Online-Befragung bestätigte:

„Ja wie gesagt, ich bin da jetzt viel offener, ich bin - ich traue mir das viel schneller zu und ja forschen ist gut und die Kinder lieben das einfach.“ 
In allen drei untersuchten Bereichen, der Selbstwirksamkeitserwartung, der Anzahl wiederholter Beispielexperimente sowie der Anzahl umgesetzter Forschungsaktivitäten, zeigen sich zwischen den vier Gruppen Unterschiede. Post-hoc-Analysen (Hochberg) belegen, dass ein signifikanter Unterschied zwischen den Gruppen mit ein bis zwei und sieben bis neun Fortbildungsbesuchen in Bezug auf die Selbstwirksamkeitserwartung besteht (.021).

In Bezug auf die wiederholten Beispielexperimente ergeben sich signifikante Gruppenunterschiede (Games-Howell) zwischen Personen mit ein bis zwei Fortbildungsbesuchen und denen mit drei bis sechs, sieben bis neun beziehungsweise mehr als neun Fortbildungsbesuchen (immer .000). Signifikant unterscheiden sich auch die Gruppen mit drei bis sechs und sieben bis neun Fortbildungsbesuchen (.000).

In Bezug auf die Häufigkeit des Forschens in der Praxis zeigen sich signifikante Unterschiede in den Post-hoc-Analysen (Games-Howell) zwischen der Gruppe mit einem bis zwei Fortbildungsbesuchen und sieben bis neun (.000) sowie mehr als neun Fortbildungsbesuchen (.004). Es finden sich aber auch Unterschiede zwischen der Gruppe mit drei bis sechs Fortbildungen und der Gruppe mit sieben bis neun Fortbildungen (.000) sowie der Gruppe mit mehr als neun Fortbildungen (.046).

Tabelle 11. Einfluss der Fortbildungsanzahl auf Selbstwirksamkeitserwartung, Anzahl wiederholter Experimente und Anzahl umgesetzter Forschungsaktivitäten (Items 3.1-3.6, 7.4 und 8.23)

\begin{tabular}{lcccccc}
\hline $\begin{array}{l}\text { Anzahl besuchter } \\
\text { Fortbildungen }\end{array}$ & $\begin{array}{c}1-2 \\
(\mathrm{n}=123-138)\end{array}$ & $\begin{array}{c}3-6 \\
(\mathrm{n}=134-140)\end{array}$ & $\begin{array}{c}7-9 \\
(\mathrm{n}=45-47)\end{array}$ & $\begin{array}{c}19 \\
(\mathrm{n}=46-48)\end{array}$ & $\mathrm{F}$ & Sig. \\
\hline $\begin{array}{l}\text { Selbstwirksam- } \\
\text { keitserwartung }\end{array}$ & 18.8 & 19.3 & 20.3 & 20.2 & $5.2^{\star}$ & .002 \\
\hline $\begin{array}{l}\text { Anzahl wiederhol- } \\
\text { ter Beispielexperi- } \\
\text { mente }\end{array}$ & 2.4 & 2.5 & 3.0 & 2.7 & $15.3^{\star}$ & .000 \\
\hline $\begin{array}{l}\text { Anzahl umgesetz- } \\
\text { ter Forschungsakti- } \\
\text { vitäten }\end{array}$ & 3.8 & 4.7 & 5.2 & 5.0 & $11.1^{\star}$ & .000 \\
\hline
\end{tabular}

einfaktorielle Varianzanalyse, ${ }^{\star} p<.05$, zweiseitig

Damit spielten die besuchten Fortbildungen für die Motivationssteigerung eine bedeutende Rolle. Bei genauerer Betrachtung lässt sich erkennen, dass die Werte von der ersten bis zur dritten Gruppe kontinuierlich ansteigen, danach aber in der Gruppe der Befragten, die mehr als neun Fortbildungen besucht haben, leicht zurückgehen (siehe Tabelle 11). 


\section{In Bezug auf ihre Einstellungen}

Die Einstellung bezüglich der Wichtigkeit der Förderung von MINT-Themen im Kindergarten und Grundschulbereich wurde im Online-Fragebogen mit den Items 2.1 und 2.2 erfasst.

\section{Berufserfahrung}

Betrachtet man die Einstellung zur Wichtigkeit des Förderbereiches MINT unterschieden nach der Berufserfahrung in Jahren, so können keine signifikanten Unterschiede zwischen den Pädagoginnen und Pädagogen festgestellt werden.

Tabelle 12. Einschätzung der Wichtigkeit des Förderbereichs MINT getrennt nach Berufserfahrung (Items 2.1 und 2.2)

\begin{tabular}{|c|c|c|c|c|c|}
\hline Berufserfahrung in Jahren & $\begin{array}{c}0-2 \\
(n=8)\end{array}$ & $\begin{array}{c}3-10 \\
(n=87)\end{array}$ & $\begin{array}{c}>10 \\
(n=254)\end{array}$ & $\mathrm{F}$ & Sig. \\
\hline $\begin{array}{l}\text { Der Förderbereich MINT ist in } \\
\text { Kindertagesstätten sehr wichtig. }\end{array}$ & 3.5 & 3.4 & 3.4 & 0.2 & .823 \\
\hline $\begin{array}{l}\text { Der Förderbereich MINT ist in Grundschulen } \\
\text { sehr wichtig. }\end{array}$ & 3.7 & 3.7 & 3.7 & 0.1 & .943 \\
\hline
\end{tabular}

\section{MINT-Hintergrund}

Es lässt sich ein signifikanter Unterschied zwischen Pädagoginnen und Pädagogen mit und ohne MINT-Hintergrund hinsichtlich der Einschätzung der Wichtigkeit des Förderbereichs MINT in Kindertagesstätten feststellen: Fach- und Lehrkräfte mit MINT-Hintergrund hielten die Förderung in diesem Bereich für signifikant wichtiger. Hinsichtlich der Einstellungen zur Wichtigkeit der MINT-Förderung im Grundschulbereich ist diese unterschiedliche Einschätzung nicht zu erkennen.

Tabelle 13. Einschätzung der Wichtigkeit des Förderbereichs MINT unterteilt nach MINTHintergrund (Items 2.1 und 2.2)

\begin{tabular}{|c|c|c|c|c|}
\hline & $\begin{array}{c}\text { MINT- } \\
\text { Hintergrund } \\
(\mathrm{n}=127-132)\end{array}$ & $\begin{array}{l}\text { Kein MINT- } \\
\text { Hintergrund } \\
(\mathrm{n}=212-239)\end{array}$ & $t$ & Sig. \\
\hline $\begin{array}{l}\text { Der Förderbereich MINT ist in } \\
\text { Kindertagesstätten sehr wichtig. }\end{array}$ & 3.6 & 3.3 & $2.84^{\star}$ & .005 \\
\hline $\begin{array}{l}\text { Der Förderbereich MINT ist in Grundschu- } \\
\text { len sehr wichtig. }\end{array}$ & 3.7 & 3.6 & 1.12 & .263 \\
\hline
\end{tabular}

\section{Anzahl der besuchten Fortbildungen}

Unterteilt man die Einschätzung der Wichtigkeit des Förderbereichs MINT im Kindergarten und in der Grundschule nach Anzahl der besuchten Fortbildungen, 
zeigen sich signifikante Unterschiede nur in Bezug auf Kindertagesstätten. Diese bestehen laut Post-hoc-Analysen (Games-Howell) zwischen der Gruppe der Befragten, die bis zu zwei Fortbildungen besucht haben, und jener Gruppe der Befragten, die bereits an sieben bis neun (.000) Fortbildungen teilgenommen haben. Signifikant unterscheidet sich auch die Gruppe mit drei bis sechs von der Gruppe mit sieben bis neun Fortbildungsbesuchen (.000).

Tabelle 14. Einschätzung der Wichtigkeit des Förderbereichs MINT unterteilt nach der Anzahl der besuchten Fortbildungen (Items 2.1 und 2.2)

\begin{tabular}{lcccccc}
\hline $\begin{array}{l}\text { Anzahl besuchter } \\
\text { Fortbildungen }\end{array}$ & $\begin{array}{c}0-2 \\
(\mathrm{n}=123-138)\end{array}$ & $\begin{array}{c}3-6 \\
(\mathrm{n}=134-140)\end{array}$ & $\begin{array}{c}7-9 \\
(\mathrm{n}=45-47)\end{array}$ & $\begin{array}{c}19 \\
(\mathrm{n}=46-48)\end{array}$ & $\mathrm{F}$ & Sig. \\
\hline $\begin{array}{l}\text { Der Förderbereich } \\
\text { MINT ist in Kinder- } \\
\text { tagesstätten sehr } \\
\text { wichtig. }\end{array}$ & 3.3 & 3.4 & 3.8 & 3.4 & $5.4^{\star}$ & .001 \\
\hline $\begin{array}{l}\text { Der Förderbereich } \\
\begin{array}{l}\text { MINT ist in Grund- } \\
\text { schulen sehr } \\
\text { wichtig. }\end{array}\end{array} \quad 3.6$ & 3.7 & 3.8 & 3.7 & 2.6 & .049 \\
\hline
\end{tabular}

einfaktorielle Varianzanalyse, *p $<.05$, zweiseitig

\section{Zusammenfassung FF U2}

Unterschiede zwischen erfahrenen und weniger erfahrenen Fach- und Lehrkräften finden sich entlang ihrer Berufserfahrung, ihres MINT-Hintergrunds und der Anzahl der von ihnen besuchten Fortbildungen:

Mit der Berufserfahrung zeigten sich signifikante Unterschiede zwischen den Gruppen hinsichtlich der Selbstwirksamkeitserwartung der Befragten in Bezug darauf, zu MINT-Themen mit den Kindern zu forschen. Dabei fielen die Werte für die Gruppen mit mehr Berufserfahrung jeweils höher aus. Dies ergab sich auch für die Häufigkeit, im MINT-Bereich zu forschen. Keine Unterschiede zeigten sich in Bezug auf das Professionswissen. Hier scheint die Berufserfahrung keinen Einfluss auf das selbsteingeschätzte Können zu haben.

Personen mit MINT-Hintergrund fühlten sich laut eigener Aussagen kompetenter, beispielhafte Phänomene den Kindern zu erklären, sind motivierter, MINTSachverhalte zu vermitteln, besitzen eine höhere Selbstwirksamkeitserwartung und haben häufiger im MINT-Bereich mit den Kindern geforscht.

Die größten Unterschiede finden sich zwischen den Gruppen in Hinblick auf die Anzahl der besuchten Fortbildungen: Zwischen pädagogischen Fach- und Lehrkräften, die eine bis zwei, drei bis sechs, sieben bis neun oder mehr als neun Fortbildungen besucht haben, finden sich signifikante Unterschiede hinsichtlich der Selbsteinschätzung ihres Professionswissens, ihrer Motivation, ihrer Selbstwirksamkeitserwartung, ihrer Einstellungen zu MINT sowie der Anzahl von For- 
schungsaktivitäten. Ein Sättigungseffekt könnte ab neun besuchten Fortbildungen eintreten, denn für diese Gruppe steigen die Mittelwerte nicht mehr an.

\subsubsection{FF U3 Gibt es Unterschiede zwischen pädagogischen Fachkräften und Grundschullehrkräften?}

Für die Beantwortung der dritten Unterfrage der Forschungsfrage werden Unterschiede zwischen Fach- und Lehrkräften analysiert, die sich aus den erhobenen Fragebogendaten ergeben, denn in den Interviews lassen sich keine deutlichen Unterschiede zwischen den Einschätzungen von Fach- und Lehrkräften finden.

\section{Besuchte Fortbildungen in der Initiative „Haus der kleinen Forscher“}

Vergleicht man die Studienteilnehmerinnen und -teilnehmer hinsichtlich der Anzahl der besuchten Fortbildungen, so besuchten pädagogische Fachkräfte, die in einer Kindertagesstätte arbeiten, durchschnittlich eine halbe Fortbildung mehr als solche, die im Hort oder als Lehrkräfte arbeiten.

Während bei den Lehrkräften die meisten weniger als drei Fortbildungen besucht haben, gab der größte Teil der Fachkräfte in Kita und Hort an, drei bis sechs Fortbildungen besucht zu haben. Erwartungsgemäß sind die Besuche der Fortbildungen zu den Themen Wasser und Luft insgesamt deutlich überrepräsentiert, weil diese im Fortbildungsprogramm der Initiative als Basisfortbildungen deklariert werden, wobei gleichwohl alle Fortbildungsinhalte vertreten sind. Deutlich wurde aber auch, dass 87 Prozent aller Befragten die Online-Angebote der Stiftung „Haus der kleinen Forscher“ noch nie genutzt haben, unabhängig von ihrer Berufszugehörigkeit.

Tabelle 15. Anzahl der besuchten Fortbildungen nach der Einrichtung

\begin{tabular}{lccc}
\hline Anzahl an besuchten Fortbildungen & $\begin{array}{c}\text { Fachkraft Kinder- } \\
\text { tagesstätte } \\
(\mathrm{n}=261)\end{array}$ & $\begin{array}{c}\text { Fachkraft Hort } \\
(\mathrm{n}=29)\end{array}$ & $\begin{array}{c}\text { Lehrkraft } \\
(\mathrm{n}=66)\end{array}$ \\
\hline 0 bis 2 & $88(34 \%)$ & $11(38 \%)$ & $37(56 \%)$ \\
\hline 3 bis 6 & $109(42 \%)$ & $12(41 \%)$ & $21(32 \%)$ \\
\hline 7 bis 9 & $35(13 \%)$ & $3(10 \%)$ & $5(8 \%)$ \\
\hline mehr als 9 & $23(9 \%)$ & $3(10 \%)$ & $3(5 \%)$ \\
\hline $\begin{array}{l}\text { Anzahl an Fortbildungen } \\
\text { (Mittelwert) }\end{array}$ & 4.2 & 3.5 & 3.6 \\
\hline
\end{tabular}

17 Die Unterscheidung in pädagogische Fachkräfte Kita und Hort sowie Lehrkräfte findet hier nach der angegebenen Einrichtung statt, nicht nach der angegebenen Profession, da dort sowohl Personen mit einer Lehramtsausbildung als auch mit einer sozialpädagogischen Ausbildung arbeiten können. 


\section{In Bezug auf das Professionswissen}

Hinsichtlich der selbsteingeschätzten Kompetenz, den „Forschungskreis“ (vgl. Marquardt-Mau, 2004, 2011; Rank et al., 2018; Stiftung Haus der kleinen Forscher, 2013) anwenden zu können, schätzten sich die Fachkräfte durchweg etwas kompetenter ein als die Lehrkräfte; signifikant werden die Unterschiede aber nur beim Item „Ideen der Kinder aufgreifen“ (siehe Tabelle 16).

Tabelle 16. Unterschied zwischen den Professionen in Bezug auf die Kompetenzen, den „Forschungskreis“ umsetzen zu können (Items 8.3, 8.6, 8.9, 8.12, 8.15, 8.18, 8.21)

\begin{tabular}{lcccc}
\hline & $\begin{array}{c}\text { Pädagogische } \\
\text { Fachkraft } \\
(\mathrm{n}=266-274)\end{array}$ & $\begin{array}{c}\text { Lehrkraft } \\
(\mathrm{n}=57-59)\end{array}$ & $\mathrm{t}$ & Sig. \\
\hline Interessen der Kinder erkennen & 8.4 & 8.2 & 1.5 & .120 \\
\hline Vorkenntnisse der Kinder einschätzen & 8.1 & 7.9 & 1.3 & .197 \\
\hline Experimente vorbereiten & 8.6 & 8.4 & 0.9 & .345 \\
\hline Anregende Fragen stellen & 8.3 & 8.0 & 1.9 & .063 \\
\hline Ideen der Kinder aufgreifen & 8.6 & 8.1 & $2.7^{\star}$ & .006 \\
\hline Kindgerechtes Erklären & 8.1 & 8.2 & -0.4 & .737 \\
\hline Dokumentieren & 7.9 & 7.8 & 0.5 & .605 \\
\hline t-Test, ${ }^{*} p<.05$ & & & &
\end{tabular}

\section{In Bezug auf ihre Motivation}

Unterschiede in der Motivation zeigen sich zwischen Fach- und Lehrkräften an verschiedenen Stellen: Lehrkräfte verfügten über eine signifikant höhere Selbstwirksamkeitserwartung als Fachkräfte. Dagegen haben Fachkräfte signifikant häufiger Beispielexperimente wiederholt. ${ }^{18}$ Kein signifikanter Unterschied zwischen Fach- und Lehrkräften ergab sich jedoch hinsichtlich der Anzahl umgesetzter Forschungsaktivitäten in der Einrichtung (siehe Tabelle 17).

18 Dabei ist zu berücksichtigen, dass pädagogische Fachkräfte auch im Schnitt häufiger Fortbildungen besucht haben und damit mehr Beispielexperimente zum Wiederholen mitnehmen konnten (vgl. Abschnitt $A$ 7.2.1). 
Tabelle 17. Unterschied zwischen der Profession in Bezug auf Selbstwirksamkeitserwartung, Anzahl umgesetzter Forschungsaktivitäten und Anzahl wiederholter Beispielexperimente (Items 3.1-3.6; 7.4 und 8.23)

\begin{tabular}{lcccc}
\hline & $\begin{array}{c}\text { Pädagogische } \\
\text { Fachkraft } \\
(\mathrm{n}=279-293)\end{array}$ & $\begin{array}{c}\text { Lehrkraft } \\
(\mathrm{n}=63-70)\end{array}$ & $\mathrm{t}$ & Sig. \\
\hline Selbstwirksamkeitserwartung & 19.2 & 20.0 & $-2.0^{*}$ & .048 \\
\hline Anzahl umgesetzter Forschungsaktivitäten & 4.5 & 4.4 & 0.2 & .836 \\
\hline Anzahl wiederholter Beispielexperimente & 2.6 & 2.4 & $2.4^{*}$ & .015 \\
\hline t-Test, ${ }^{*} p<.05$ & & & &
\end{tabular}

\section{In Bezug auf ihre Einstellungen}

Betrachtet man die Einstellungen pädagogischer Fach- und Lehrkräfte zur Wichtigkeit des Förderbereichs MINT, so sind keine signifikanten Unterschiede festzustellen, lediglich Tendenzen (Tabelle 18). Auffallend ist, dass Fach- und Lehrkräfte die Förderung von MINT-Themen in Grundschulen als etwas wichtiger erachtet als in Kindertagestätten (siehe auch Abschnitt A 8.1.1).

Tabelle 18. Unterschiede in den Einstellungen pädagogischer Fach- und Lehrkräfte zum Förderbereich MINT (Item 2.1 und 2.2)

\begin{tabular}{lcccc}
\hline & $\begin{array}{c}\text { Lehrkraft } \\
(\mathrm{n}=64-68)\end{array}$ & $\begin{array}{c}\text { Pädagogische } \\
\text { Fachkraft } \\
(\mathrm{n}=271-300)\end{array}$ & $\mathrm{t}$ & Sig. \\
\hline $\begin{array}{l}\text { Der Förderbereich MINT ist in Kindertages- } \\
\text { stätten sehr wichtig. }\end{array}$ & 3.3 & 3.4 & 1.90 & .058 \\
\hline $\begin{array}{l}\text { Der Förderbereich MINT ist in Grundschulen } \\
\text { sehr wichtig. }\end{array}$ & 3.7 & 3.7 & 0.28 & .782 \\
\hline
\end{tabular}

$\mathrm{t}$-Test, ${ }^{*} \mathrm{p}<.05$

\section{Zusammenfassung $\mathrm{FF} \mathrm{U}_{3}$}

Im Datensatz lassen sich nur wenige Unterschiede zwischen Fach- und Lehrkräften erkennen. Lehrkräfte und pädagogische Fachkräfte schätzen sich in ihrem Professionswissen nur hinsichtlich des Aufgreifens von Ideen signifikant unterschiedlich ein - hier fühlen sich die Fachkräfte kompetenter. Hinsichtlich der Motivation fällt auf, dass die pädagogischen Fachkräfte signifikant häufiger an Fortbildungen teilgenommen haben und auch häufiger Beispielexperimente aus diesen in der Einrichtung durchgeführt haben. Die Lehrkräfte haben dagegen eine höhere Selbstwirksamkeitserwartung. Bezüglich der Einstellungen konnten auf Einzelitemebene keine signifikanten Unterschiede zwischen den Professionsgruppen festgestellt werden. 
Diese Ergebnisse finden sich in den Indizes überwiegend wieder: Während es beim Index „Motivation“ keinen Unterschied gibt, schätzten die Fachkräfte ihr Professionswissen insgesamt signifikant besser ein als die Lehrkräfte. Der Index „Einstellungen“ zeigt einen signifikanten Unterschied zwischen den Berufsgruppen, was auf der Einzelitemebene nicht zu finden ist. Da aber dieser Index einen wenig annehmbaren Cronbach-Alpha-Wert aufweist, ist dieses abweichende Ergebnis vermutlich darauf zurückzuführen (siehe Tabelle 19).

Tabelle 19. Unterschiede zwischen den Professionen in Bezug auf die Indizes „Professionswissen“, „Motivation“ und „Einstellungen“

\begin{tabular}{lcccc}
\hline & $\begin{array}{c}\text { Pädagogische } \\
\text { Fachkraft } \\
(\mathrm{n}=198-254)\end{array}$ & $\begin{array}{c}\text { Lehrkraft } \\
(\mathrm{n}=42-57)\end{array}$ & $\mathrm{t}$ & Sig. \\
\hline Index „Professionswissen“ & 126.5 & 121.0 & $2.5^{\star}$ & .005 \\
\hline Index „Motivation“ & 42.2 & 43.6 & -1.3 & .202 \\
\hline Index „Einstellungen“ & 10.1 & 9.6 & $1.9^{\star}$ & .015 \\
\hline
\end{tabular}

$\mathrm{t}$-Test, ${ }^{\star} \mathrm{p}<.05$

\subsubsection{FF U4 Wie lassen sich die Qualifizierungsbedarfe beschreiben?}

Über die Hälfte der Online-Befragten gab an, dass sie ihre Rolle als Lernbegleitung ohne die Fortbildungen der Initiative „Haus der kleinen Forscher“ nicht sehr gut hätten erfüllen können (Item 6.1 und 6.2). Hierbei lässt sich ein signifikanter Unterschied (.001) zwischen den Professionen in der Wahrnehmung ihrer Rolle als Lernbegleitung feststellen: Demnach meinten die Fachkräfte (MW 2,3, SD 0,7), dass sie ohne die Fortbildungen der Initiative „Haus der kleinen Forscher“ die Rolle als Lernbegleitung weniger gut bestreiten könnten, als das die Lehrkräfte einschätzten (MW 2,7, SD 0,7). Insgesamt berichten 83 Prozent, ihr Wissen im MINT-Bereich hätte sich durch die Fortbildungen der Initiative „Haus der kleinen Forscher" erhöht (Item 6.3). 92 Prozent gaben an, dort Wichtiges für die Arbeit in der Kita beziehungsweise Grundschule gelernt zu haben (Item 6.6), und 75 Prozent meinten, dass sich dies auch auf den Forscherdrang der Kinder auswirke (Item 6.5).

Sowohl die Interviews als auch die Fragebögen geben Aufschluss darüber, welche weiteren Qualifizierungsbedarfe die pädagogischen Fach- und Lehrkräfte sahen. Sie werden im Folgenden nacheinander dargestellt.

Auf die Frage, was in den Fortbildungen besonders motivierend war, äußerten viele im Interview, dass dort Anregungen gegeben wurden, die leicht umsetzbar seien, und dass selbst geforscht wurde. Außerdem äußerten viele Befragte, die Fortbildungen würden „mitreißen“ und man gerate ins Staunen. Im Großen und 
Ganzen überwiegen die positiven Beurteilungen der Fortbildungen (siehe Abbildung 13).

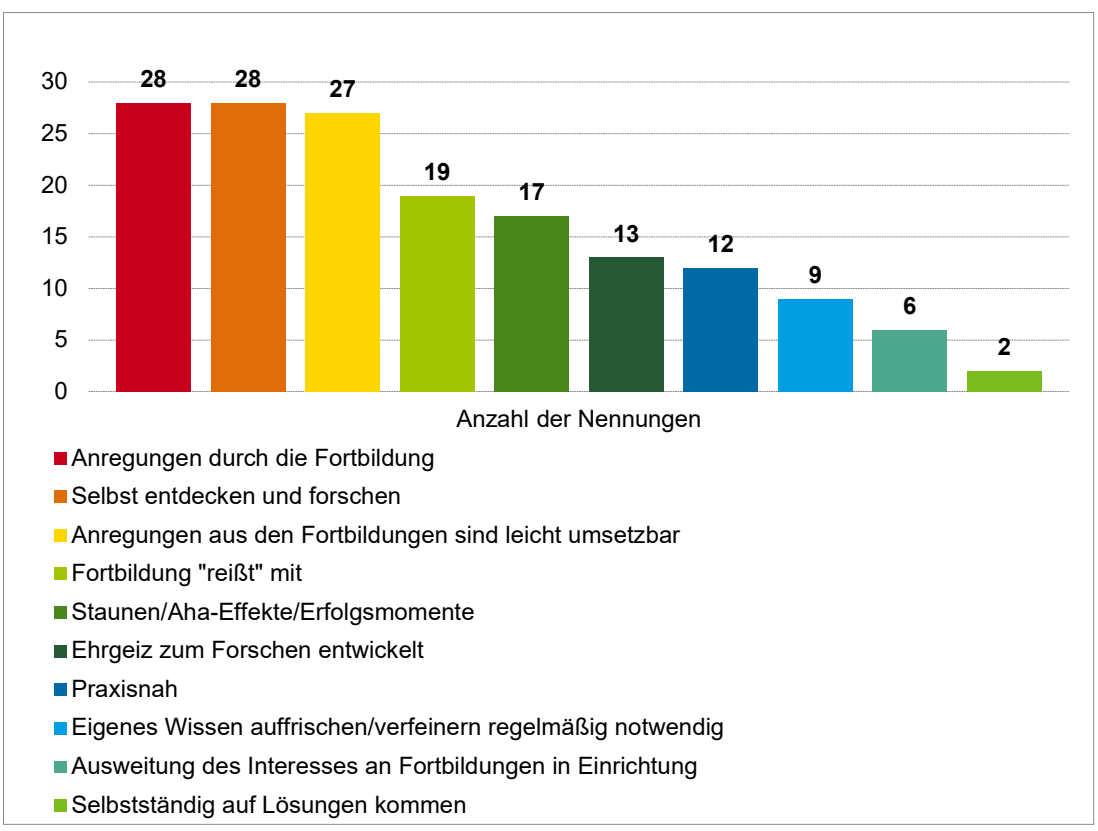

Abbildung 13. Motivationsfördernde Aspekte der Fortbildungen (Interviewbefragung)

Auf die Frage, was als demotivierend empfunden wurde, äußerte ein Teil der Interviewten, dass in einigen Fortbildungen die Themen zu kompliziert bearbeitet wurden; als ein negatives Beispiel wurde die Mathematik-Fortbildung genannt, die zu schwer gewesen sei und nicht optimal am Vorwissen der Teilnehmerinnen und Teilnehmer angeknüpft hätte. Weiter wurde kritisiert, dass das Alter der Kinder nicht ausreichend berücksichtigt würde.

Den meisten Interviewpartnerinnen und -partnern fiel es schwer, weitere Themen zu nennen, zu denen sie sich gerne fortbilden lassen würden. Die meisten äußerten stattdessen, die Themen der Bildungsangebote der Stiftung „Haus der kleinen Forscher" seien vielfältig und ausreichend. Sie schätzten außerdem den regelmäßigen Angebotsturnus der Fortbildungen und die Möglichkeit, auch mehrmals zum gleichen Thema zu erscheinen, weil jede Fortbildung stets überarbeitet und weiterentwickelt werde.

In eine ganz ähnliche Richtung gehen die Antworten der Online-Befragung. Die Hälfte der Befragten legte Wert darauf, das bisherige Format genauso beizubehalten. 45 Prozent aller Befragten wünschten sich noch mehr konkrete Tipps zum Forschen mit Kindern. 24 Prozent hätten gerne ihre Rolle als Lernbegleitung stärker behandelt. Etwa ein Fünftel wünschte sich ein breiteres Themenangebot, 
12 Prozent hätten gerne mehr Gelegenheit zum Austausch. Nur wenige, nämlich 8 Prozent, wünschten sich mehr theoretischen Input dazu, warum was wie funktioniert, und 5 Prozent hätten den „Forschungskreis“ (vgl. Marquardt-Mau, 2004, 2011; Rank et al., 2018; Stiftung Haus der kleinen Forscher, 2013) gerne detaillierter behandelt (Item 11.1, Mehrfachantworten möglich).

Betrachtet man die Wünsche bezüglich der Fortbildungen jedoch getrennt nach der Profession, so sind einige Unterschiede erkennbar (Tabelle 20). Die Lehrkräfte scheinen mit dem bisherigen Konzept der Fortbildungen etwas unzufriedener als die Fachkräfte. Ergänzend erwähnten die Fachkräfte häufiger, dass vermehrt unterschiedliche Altersstufen berücksichtigt werden und vermehrt die Möglichkeit zum Austausch mit anderen Pädagoginnen und Pädagogen gegeben wird.

Tabelle 20. Wünsche der pädagogischen Fach- und Lehrkräfte bezüglich der zukünftigen Fortbildungen vom „Haus der kleinen Forscher“ (Item 11.1)

\begin{tabular}{lcc}
\hline $\begin{array}{l}\text { Was wünschen Sie sich von zukünftigen Fortbildungen des } \\
\text { „Hauses der kleinen Forscher?“ Ich wünsche mir, ... }\end{array}$ & $\begin{array}{c}\text { Pädagogische } \\
\text { Fachkraft } \\
(\mathrm{n}=306)\end{array}$ & $\begin{array}{c}\text { Lehr- } \\
\text { kraft } \\
(\mathrm{n}=70)\end{array}$ \\
\hline ... dass das bisherige Konzept beibehalten wird. & $52.9 \%$ & $37.1 \%$ \\
\hline$\ldots$ dass noch mehr Theorie vermittelt wird. & $8.2 \%$ & $8.6 \%$ \\
\hline$\ldots$ dass die Rolle der Lernbegleitung stärker thematisiert wird. & $24.8 \%$ & $25.7 \%$ \\
\hline $\begin{array}{l}\text { g. dass noch mehr konkrete Tipps zum Forschen mit Kindern } \\
\text { gegeben werden. }\end{array}$ & $44.4 \%$ & $47.1 \%$ \\
\hline $\begin{array}{l}\text { besser berücksichtigt werden sollte. } \\
\text { b. dass andere Themen angeboten werden. }\end{array}$ & $38.9 \%$ & $27.1 \%$ \\
\hline$\ldots$ dass der „Forschungskreis“ detaillierter behandelt wird. & $21.2 \%$ & $11.4 \%$ \\
\hline$\ldots$ dass mehr Gelegenheit zum Austausch mit anderen Pädago- & $5.6 \%$ & $5.7 \%$ \\
\hline ginnen und Pädagogen gegeben wird. & $15 \%$ & $4.3 \%$ \\
\hline Anderes & $2.6 \%$ & $2.9 \%$ \\
\hline
\end{tabular}

Mehrfachantworten waren möglich.

In einem offenen Antwortfeld konnten noch weitere Bedarfe genannt werden. Es finden sich folgende Themen: eine stärkere Berücksichtigung der Lebenswelt der Kinder, z. B. die Entwicklung vom Analogen vermehrt hin zum Digitalen, das Thema „Informatik in der Grundschule“ und „Sexualität“, Anpassung der Themen an die Belange von Grundschülerinnen und Grundschülern sowie ein besserer Zugang zu weiterführender Literatur beziehungsweise weiteren Handreichungen für die Praxis in den Einrichtungen.

Den Genderaspekt in den Fortbildungen stärker herauszustellen, wurde von den meisten Befragten (82 Prozent) als unwichtig eingeschätzt (Item 10.3). Die 
Befragten sind zu 64 Prozent der Meinung, dass Jungen und Mädchen die Experimente zu MINT-Themen gleich gut verstehen (Item 10.1).

Die Erwartungen, die die Teilnehmerinnen und Teilnehmer an die Fortbildungen der Initiative „Haus der kleinen Forscher“ haben (Item 5.1), decken sich weitestgehend zwischen Fach- und Lehrkräften. Lediglich in drei Bereichen unterscheiden sie sich: Erstens wurden direkt einsetzbare Materialien von 70 Prozent der Fachkräfte und 83 Prozent der Lehrkräfte gefordert. Damit ist der entsprechende Anteil bei den Lehrkräften höher. Zweitens erwarteten nur 49 Prozent der Lehrkräfte eine Vermittlung von Fachwissen, während die Fachkräfte zu 66 Prozent auf eine solche abzielten. Der größte Unterschied bei den Erwartungen an die Fortbildungen besteht aber drittens in dem Umstand, dass 70 Prozent der Fachkräfte sich einen Erfahrungsaustausch mit anderen Pädagoginnen und Pädagogen wünschten, während der Anteil der Lehrkräfte nur bei 47 Prozent lag.

Weiter wurde im Online-Fragebogen erfragt, welche der Rahmenbedingungen der Fortbildungen der Initiative „Haus der kleinen Forscher“ die pädagogischen Fachkräfte und Lehrkräfte als besonders förderlich für ihre persönliche Weiterqualifizierung empfinden (Item 7.2). Durch die Trennung in die Professionsgruppen ist zu erkennen, dass sich Fach- und Lehrkräfte in ihrer Einschätzung nur wenig unterscheiden (siehe Abbildung 14). Als am wichtigsten werden mit Abstand die Verknüpfung von Theorie und Praxis in der Fortbildung empfunden sowie das regelmäßige und vielfältige Angebot der Fortbildungen.

Darüber hinaus wurde im Online-Fragebogen gefragt, welche Aspekte aus den Fortbildungen besonders hilfreich für die Umsetzung von Experimenten beziehungsweise Forschungsanregungen im MINT-Bereich in der Einrichtung waren (Item 8.25). Auch hier sind mit zwei Ausnahmen keine größeren Unterschiede zwischen pädagogischen Fach- und Lehrkräften zu erkennen (siehe Abbildung 15). Mit 23 Prozent scheint es die pädagogischen Fachkräfte mehr als die Lehrkräfte (7 Prozent) zu erleichtern, dass sie nicht alles selbst wissen müssen, um mit den Kindern forschen zu können. Auch dass die Lernprozesse der Kinder immer im Zentrum der Fortbildungen stehen, empfanden die pädagogischen Fachkräfte (20 Prozent) unterstützender als die Lehrkräfte. Als besonders hilfreich sticht heraus, dass die zum Forschen verwendeten Materialien alltagstauglich und günstig waren sowie Material und Anregungen mit nach Hause gegeben wurden. Auffällig ist außerdem, dass nur 29 Prozent der Lehrkräfte und 34 Prozent der pädagogischen Fachkräfte es als hilfreich empfanden, dass sie bei den Fortbildungen ohne Anleitung selbst entdecken und forschen konnten. Zudem wurden in den Interviews teilweise Hemmungen („sich darauf einlassen“) beziehungsweise Verunsicherung bezüglich des selbstständigen Forschens und Entdeckens berichtet. 


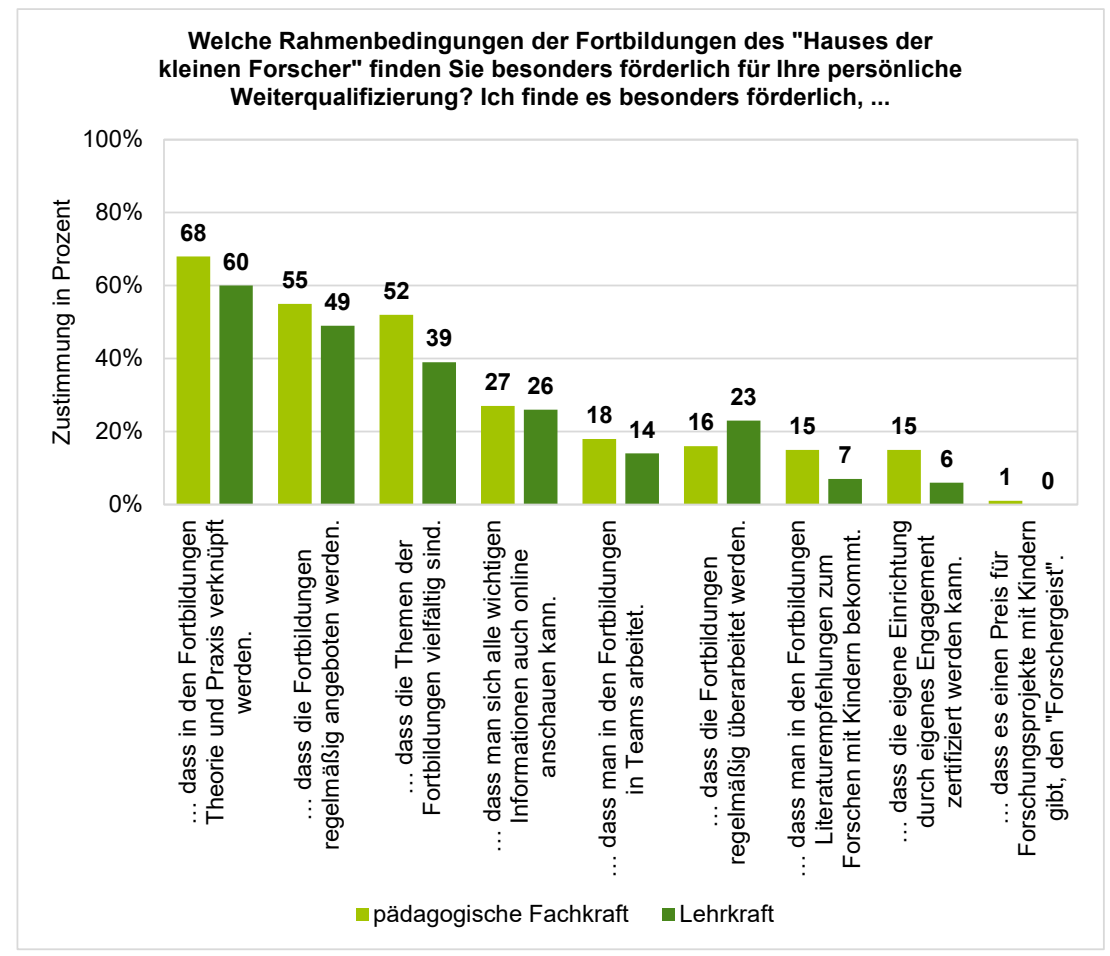

Abbildung 14. Förderliche Rahmenbedingungen der Fortbildungen der Initiative „Haus der kleinen Forscher" für die persönliche Weiterqualifizierung der pädagogischen Fach- und Lehrkräfte (Item 7.2) 


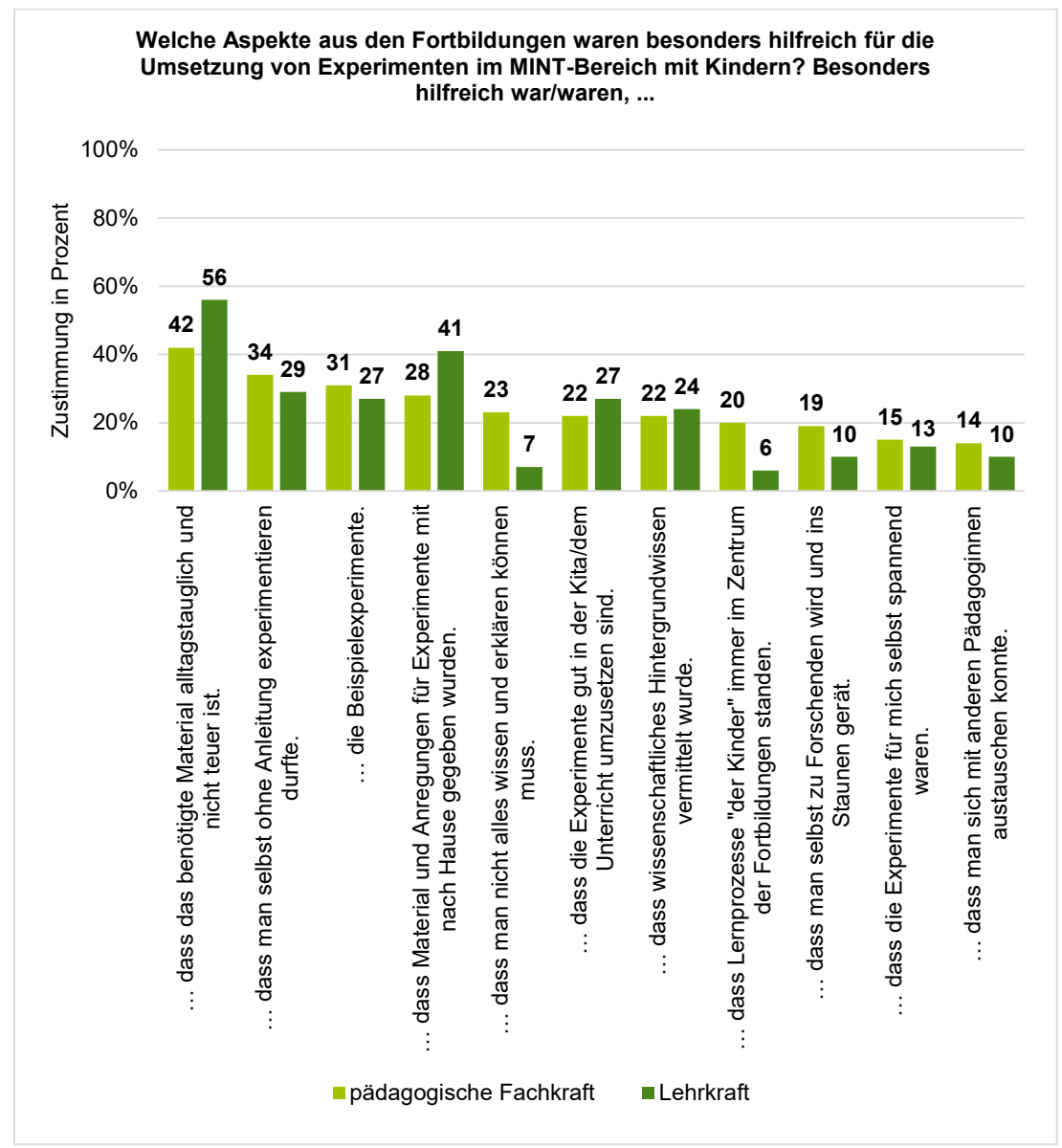

Abbildung 15. Hilfreiche Aspekte aus den Fortbildungen für die Umsetzung von Experimenten im MINT-Bereich mit Kindern (Item 8.25)

\section{Zusammenfassung $\mathrm{FF} \mathrm{U}_{4}$}

Die MINT-Fortbildungen der Initiative wurden überwiegend positiv eingeschätzt. Als motivierend wurden sie insbesondere aufgrund ihrer Praxisnähe, konkreten Tipps zum Forschen mit Kindern, leicht umsetzbaren Beispielexperimenten und Austauschgelegenheiten mit anderen Pädagoginnen und Pädagogen beschrieben.

Qualifizierungsbedarfe bestünden nach Selbstaussage der Befragten, zum einen bezüglich eines angemessenen Umgangs beim Forschen mit Kindern unterschiedlicher Altersstufen und zum anderen seien vermehrt konkrete Tipps zum Forschen und zur Rolle der Lernbegleitung seitens der Befragten erwünscht. Insgesamt wurde das bisherige Konzept der Stiftung „Haus der kleinen Forscher“ von den Befragten aber als gewinnbringend eingeschätzt und nur wenig kritisiert. 
50 Prozent der Befragten wollten das bisherige Konzept beibehalten und sahen keinen Verbesserungsbedarf, 45 Prozent der Befragten wünschten sich noch mehr konkrete Tipps zum Forschen mit Kindern. Insgesamt wurden schnell umsetzbare Praxisempfehlungen von den Teilnehmerinnen und Teilnehmern mehr geschätzt als tiefere Einblicke in naturwissenschaftliche und didaktische Theorien.

\subsection{Entwicklungsverläufe im Professionalisierungsprozess - Beantwortung der Hauptforschungsfragen}

Um abschließend zur Beantwortung der Hauptforschungsfragen Aussagen über Entwicklungsverläufe treffen zu können, sollen im nächsten Schritt Verläufe über Hilfskonstruktionen verdeutlicht werden, indem die Entwicklungen zum einen über retrospektive Einschätzungen abgebildet und zum anderen die Ergebnisse weniger erfahrener und erfahrenerer Pädagoginnen und Pädagogen systematisch verglichen werden, wie es auch zur Beantwortung der FF U2 (Kapitel 8.1.2) bereits erfolgte.

\subsubsection{FF1 Wie lassen sich die Entwicklungsverläufe von pädagogischen Fach- und Lehrkräften in Bezug auf die Professionalisierung im MINT-Bereich beschreiben?}

Zunächst wurde die Entwicklung der Fach- und Lehrkräfte hinsichtlich der Veränderung ihres Professionswissens durch eine retrospektive Einschätzung nachgezeichnet. Um die professionelle Entwicklung hinsichtlich der Nutzung des „Forschungskreises“ (vgl. Marquardt-Mau, 2004, 2011; Rank et al., 2018; Stiftung Haus der kleinen Forscher, 2013) als Teil des fachdidaktischen Wissens beurteilen zu können, wurden die Items 8.1 bis 8.21 genutzt, in denen nach Unterschieden in der Kompetenzwahrnehmung zu unterschiedlichen Zeitpunkten gefragt wurde. Im Allgemeinen kann man beobachten, dass sich die Lehrkräfte direkt nach der Ausbildung signifikant besser einschätzten als die pädagogischen Fachkräfte. Diesen „Rückstand“ holten die pädagogischen Fachkräfte nach eigener Aussage jedoch auf und schätzten sich nach der letzten Fortbildung durchgehend kompetenter ein als die Lehrkräfte. Signifikante Unterschiede zwischen den Einschätzungen gibt es nur vereinzelt. Lehrkräfte schätzten sich im Rückblick direkt nach der Ausbildung hinsichtlich des Erkennens von Interessen der Kinder, im Stellen anregender Fragen sowie im Dokumentieren von Experimenten signifikant besser ein als pädagogische Fachkräfte. Lehrkräfte und pädagogische Fachkräfte unterscheiden sich zudem hinsichtlich der Kompetenzfacette „Kindgerechtes Erklären von Naturphänomenen“ direkt nach der Ausbildung, aber auch nach der ersten Fortbildung 
signifikant voneinander - Lehrkräfte schätzten sich zu diesen Zeitpunkten besser ein. Kindgerechtes Erklären stellt die einzige Kompetenzfacette dar, bei der sich Lehrkräfte und pädagogische Fachkräfte erst nach der letzten Fortbildung gleich kompetent einstuften. Im Aufgreifen der Ideen der Kinder schätzten sich die pädagogischen Fachkräfte nach der letzten Fortbildung signifikant besser ein als die Lehrkräfte (Tabelle 21).

Tabelle 21. Retrospektive Betrachtung der Entwicklung des fachdidaktischen Wissens (,Forschungskreis“)

\begin{tabular}{|c|c|c|c|c|}
\hline & $\begin{array}{l}\text { MW Pädagogische } \\
\text { Fachkraft (SD) }\end{array}$ & $\begin{array}{l}\text { MW Lehrkraft } \\
\text { (SD) }\end{array}$ & $\mathrm{t}$ & Sig. \\
\hline $\begin{array}{l}8.1 \text { Interessen der Kinder erkennen: } \\
\text { direkt nach der Ausbildung }\end{array}$ & $6.1(2.2)$ & $6.8(1.7)$ & $-2.4^{\star}$ & .016 \\
\hline $\begin{array}{l}8.2 \text { Interessen der Kinder erkennen: } \\
\text { nach der ersten Fortbildung }\end{array}$ & 7.7 (1.5) & $7.8(1.2)$ & -0.3 & .746 \\
\hline $\begin{array}{l}8.3 \text { Interessen der Kinder erkennen: } \\
\text { nach der letzten Fortbildung }\end{array}$ & $8.4(1.3)$ & $8.2(1.1)$ & 1.6 & .120 \\
\hline $\begin{array}{l}8.4 \text { Vorkenntnisse der Kinder einschät- } \\
\text { zen: direkt nach der Ausbildung }\end{array}$ & $5.9(2.1)$ & $6.4(1.8)$ & -1.7 & .099 \\
\hline $\begin{array}{l}\text { 8.5 Vorkenntnisse der Kinder einschät- } \\
\text { zen: nach der ersten Fortbildung }\end{array}$ & $7.3(1.6)$ & $7.6(1.3)$ & -1.3 & .190 \\
\hline $\begin{array}{l}\text { 8.6 Vorkenntnisse der Kinder einschät- } \\
\text { zen: nach der letzten Fortbildung }\end{array}$ & $8.1(1.3)$ & $7.9(1.3)$ & 1.3 & .197 \\
\hline $\begin{array}{l}\text { 8.7 Experimente vorbereiten: } \\
\text { direkt nach der Ausbildung }\end{array}$ & $5.7(2.5)$ & $6.5(2.1)$ & -2.2 & .026 \\
\hline $\begin{array}{l}\text { 8.8 Experimente vorbereiten: } \\
\text { nach der ersten Fortbildung }\end{array}$ & $7.7(1.6)$ & $7.9(1.5)$ & -0.8 & .439 \\
\hline $\begin{array}{l}\text { 8.9 Experimente vorbereiten: } \\
\text { nach der letzten Fortbildung }\end{array}$ & $8.6(1.3)$ & $8.4(1.2)$ & 0.9 & .345 \\
\hline $\begin{array}{l}\text { 8.10 Anregende Fragen stellen, ohne } \\
\text { die Antwort zu verraten: direkt nach der } \\
\text { Ausbildung }\end{array}$ & $5.7(2.3)$ & $6.4(2.1)$ & $-2.0^{\star}$ & .045 \\
\hline $\begin{array}{l}\text { 8.11 Anregende Fragen stellen, ohne } \\
\text { die Antwort zu verraten: nach der } \\
\text { ersten Fortbildung }\end{array}$ & $7.5(1.6)$ & $7.7(1.3)$ & -1.2 & .222 \\
\hline $\begin{array}{l}\text { 8.12 Anregende Fragen stellen, ohne } \\
\text { die Antwort zu verraten: nach der } \\
\text { letzten Fortbildung }\end{array}$ & $8.3(1.4)$ & $8(1)$ & 1.9 & .063 \\
\hline $\begin{array}{l}8.13 \text { Ideen der Kinder aufgreifen: } \\
\text { direkt nach der Ausbildung }\end{array}$ & $6.5(2.3)$ & $6.5(2.1)$ & 0.3 & .768 \\
\hline $\begin{array}{l}8.14 \text { Ideen der Kinder aufgreifen: } \\
\text { nach der ersten Fortbildung }\end{array}$ & $7.8(1.8)$ & $7.8(1.4)$ & 0.3 & .782 \\
\hline $\begin{array}{l}8.15 \text { Ideen der Kinder aufgreifen: } \\
\text { nach der letzten Fortbildung }\end{array}$ & $8.6(1.3)$ & $8.1(1.3)$ & $2.7^{\star}$ & .006 \\
\hline
\end{tabular}




\begin{tabular}{|c|c|c|c|c|}
\hline $\begin{array}{l}\text { 8.16 Kindgerechtes Erklären von Natur- } \\
\text { phänomenen: } \\
\text { direkt nach der Ausbildung }\end{array}$ & $5.6(2.2)$ & $6.4(2.1)$ & $-2.6^{*}$ & .010 \\
\hline $\begin{array}{l}8.17 \text { Kindgerechtes Erklären von Natur- } \\
\text { phänomenen: } \\
\text { nach der ersten Fortbildung }\end{array}$ & $7.3(1.7)$ & $7.8(1.5)$ & $-2.0^{*}$ & .042 \\
\hline $\begin{array}{l}8.18 \text { Kindgerechtes Erklären von } \\
\text { Naturphänomenen: nach der letzten } \\
\text { Fortbildung }\end{array}$ & $8.1(1.4)$ & $8.2(1.3)$ & -0.3 & .737 \\
\hline $\begin{array}{l}\text { 8.19 Dokumentieren von Experimentie- } \\
\text { ren und Ergebnissen: direkt nach der } \\
\text { Ausbildung }\end{array}$ & $5.4(2.5)$ & $6.1(2.1)$ & $-2.5^{\star}$ & .016 \\
\hline $\begin{array}{l}\text { 8.20 Dokumentieren von Experimentie- } \\
\text { ren und Ergebnissen: nach der ersten } \\
\text { Fortbildung }\end{array}$ & $7.2(1.9)$ & $7.5(1.5)$ & -1.7 & .099 \\
\hline $\begin{array}{l}\text { 8.21 Dokumentieren von Experimentie- } \\
\text { ren und Ergebnissen: nach der letzten } \\
\text { Fortbildung }\end{array}$ & $7.9(1.7)$ & $7.8(1.3)$ & 0.5 & .605 \\
\hline
\end{tabular}

Mit den Items 6.8 bis 6.17 sowie in Item 6.19 wurde abgefragt, was die pädagogischen Fach- und Lehrkräfte in den Fortbildungen gelernt haben, sodass nochmal explizit nach dem Mehrwert der Fortbildungen für die eigene Professionalisierung gefragt wurde (Tabelle 22 und 23). Die pädagogischen Fachkräfte schätzten ihren Wissenszuwachs durch die Fortbildungen signifikant größer ein als die Lehrkräfte. Nur beim Wissen, Kinder zum Forschen zu motivieren, und der Einschätzung, inwiefern die Fortbildungen (hilfreiche) Anleitungen bieten, wie man mit Kindern forschen sollte, ist keine unterschiedliche Einschätzung der Professionen festzustellen. Zu fachlichen Hintergründen, spielerischem Vorgehen, kindgerechtem Erklären, Anstoßen von Denkprozessen beim Kind, Kinder unterschiedlichen Alters zu unterstützen, spontan und angemessen auf Kinderfragen zu reagieren sowie mit Alltagsmaterialien zu forschen, scheinen die Fachkräfte aus den Fortbildungen dagegen mehr mitzunehmen. Angesichts dessen, dass nur 26 Prozent der pädagogischen Fachkräfte - im Gegensatz zu 74 Prozent der Lehrkräfte - einen MINT-Hintergrund haben, scheint dieser signifikante Unterschied zwischen Lehrund Fachkräften plausibel. 
Tabelle 22. Eingeschätzte Wirksamkeit der Fortbildungen (Item 6.8 bis $6.17,6.19$ )

\begin{tabular}{|c|c|c|c|c|c|}
\hline & $\begin{array}{l}\text { MW Gesamte } \\
\text { Stichprobe (SD) } \\
(n=339-360)\end{array}$ & $\begin{array}{l}\text { Pädagogische } \\
\text { Fachkraft (SD) } \\
(\mathrm{n}=275-292)\end{array}$ & $\begin{array}{l}\text { Lehrkraft (SD) } \\
\quad(n=51-61)\end{array}$ & $\mathrm{t}$ & Sig. \\
\hline Kindgerechtes Erklären & $2.7(0.8)$ & $2.8(0.8)$ & $2.4(0.7)$ & $3.183^{\star}$ & .002 \\
\hline $\begin{array}{l}\text { Hintergrundwissen zu } \\
\text { MINT-Themen }\end{array}$ & $3.1(0.7)$ & $3.2(0.7)$ & $2.8(0.8)$ & $3.929^{\star}$ & .000 \\
\hline $\begin{array}{l}\text { Kinder zum Forschen } \\
\text { motivieren }\end{array}$ & $3.2(0.7)$ & $3.2(0.7)$ & $3.1(0.7)$ & 0.438 & .661 \\
\hline $\begin{array}{l}\text { Man muss nicht alles } \\
\text { wissen, es muss nicht } \\
\text { alles funktionieren. }\end{array}$ & $3.5(0.7)$ & $3.5(0.6)$ & $3.2(0.8)$ & $3.369^{\star}$ & .001 \\
\hline $\begin{array}{l}\text { Mit kleinem Budget und } \\
\text { Alltagsmaterialien kann } \\
\text { man viel forschen und } \\
\text { Erkenntnisse gewinnen. }\end{array}$ & $3.6(0.6)$ & $3.6(0.5)$ & $3.3(0.8)$ & $3.369^{\star}$ & .001 \\
\hline $\begin{array}{l}\text { Jüngere und ältere } \\
\text { Kinder benötigen unter- } \\
\text { schiedliche Begleitung } \\
\text { beim Forschen. }\end{array}$ & $3.3(0.7)$ & $3.3(0.7)$ & $2.9(0.9)$ & $4.232^{\star}$ & .000 \\
\hline $\begin{array}{l}\text { Spielerisch die Welt } \\
\text { entdecken }\end{array}$ & $3.5(0.7)$ & $3.6(0.6)$ & $3.3(0.9)$ & $2.276^{*}$ & .026 \\
\hline $\begin{array}{l}\text { Anleitungen, wie man } \\
\text { mit Kindern forschen } \\
\text { sollte }\end{array}$ & $3.2(0.7)$ & $3.2(0.7)$ & $3(0.8)$ & 1.608 & .109 \\
\hline $\begin{array}{l}\text { Neue Ideen, die den } \\
\text { Kita-/Grundschulalltag } \\
\text { erleichtern }\end{array}$ & $3.4(0.7)$ & $3.4(0.6)$ & $3.2(0.9)$ & $2.218^{*}$ & .030 \\
\hline $\begin{array}{l}\text { Spontan und angemes- } \\
\text { sen reagieren, wenn } \\
\text { Kinder Fragen haben }\end{array}$ & $3.1(0.8)$ & $3.2(0.8)$ & $2.7(0.8)$ & $4.128^{*}$ & .000 \\
\hline $\begin{array}{l}\text { Nach den Fortbildungen } \\
\text { gelingt es mir besser, } \\
\text { bei Kindern vertiefte } \\
\text { Denkprozesse anzu- } \\
\text { stoßen. }\end{array}$ & $2.9(0.6)$ & $3(0.6)$ & $2.7(0.6)$ & $2.969^{\star}$ & .003 \\
\hline
\end{tabular}

$\mathrm{t}$-Test, ${ }^{\star} \mathrm{p}<.05$

In einem weiteren Schritt wurden die Verläufe der Kompetenzentwicklung über den Vergleich der Aussagen von erfahrenen und weniger erfahrenen Fach- und Lehrkräften nachgezeichnet. Dazu wurden die Merkmale Berufserfahrung sowie Anzahl der besuchten Fortbildungen als Vergleichsvariablen herangezogen. Der Verlauf wurde hinsichtlich der selbsteingeschätzten Veränderungen beziehungsweise Unterschiede beim Forschen mit Kindern zu MINT-Themen in den Kompetenzfacetten Professionswissen, Motivation und Einstellungen erfasst; hierfür 
wurden die Items jeweils zu einem sogenannten Index zusammengefasst (siehe Abschnitt A 7.2.2).

Werden die erstellten Indizes zu „Professionswissen“, „Motivation“ und „Einstellungen“ in Bezug auf die Berufserfahrung berechnet, ergeben sich folgende Ergebnisse, die in Tabelle 23 dargestellt sind. Der Mittelwert des Index „Professionswissen" steigt zwar mit der Berufserfahrung kontinuierlich an, jedoch sind die Differenzen zwischen den Gruppen nicht signifikant. Die kumulierten Werte zu den Aussagen der Befragten im Index „Motivation“ dagegen unterscheiden sich zwischen den Gruppen mit unterschiedlicher Berufserfahrung signifikant. Die Unterschiede liegen laut den Post-hoc-Analysen (Hochberg) zwischen den Berufsanfängerinnen und -anfängern (ein bis zwei Jahre) und den Fortgeschrittenen (drei bis zehn Jahre Berufserfahrung) (.041) sowie zwischen den Berufsanfängerinnen und -anfängern (ein bis zwei Jahre) und den Profis (über zehn Jahre Berufserfahrung) (.006). Beim Index „Einstellungen“ zeigen sich keine signifikanten Unterschiede (siehe Tabelle $23^{19}$ ).

Tabelle 23. Einfluss der Berufserfahrung auf die Indizes „Professionswissen“, „Motivation“ und „Einstellungen“

\begin{tabular}{lccccc}
\hline Berufserfahrung in Jahren & $\begin{array}{c}0-2 \\
(\mathrm{n}=5)\end{array}$ & $\begin{array}{c}3-10 \\
(\mathrm{n}=63)\end{array}$ & $\begin{array}{c}>10 \\
(\mathrm{n}=174)\end{array}$ & $\mathrm{F}$ & Sig. \\
\hline Index „Professionswissen“ & 123 & 124 & 126 & 0.6 & .559 \\
\hline Index „Motivation“ & 34 & 41 & 42 & $5.9^{\star}$ & .003 \\
\hline Index „Einstellungen“ & 10 & 10 & 10 & 0.3 & .762 \\
\hline
\end{tabular}

einfaktorielle Varianzanalyse, ${ }^{\star} p<.05$, zweiseitig

In Bezug auf die Anzahl der besuchten Fortbildungen verändern sich alle IndexWerte signifikant. Beim Index „Professionswissen“ zeigen sich im Post-hoc-Test (Hochberg) Unterschiede zwischen den Gruppen mit ein bis zwei Fortbildungsbesuchen und der Gruppe mit sieben bis neun und mehr als neun Fortbildungsbesuchen (.013 beziehungsweise .000 ) sowie zwischen der Gruppe mit drei bis sechs Fortbildungsbesuchen und der Gruppe mit mehr als neun Fortbildungen (.004).

Beim Index „Motivation“ lassen sich Signifikanzen (Games-Howell) zwischen allen Gruppen finden (.000 bis .009), bis auf die Gruppen der Befragten mit sieben bis neun Fortbildungsbesuchen und denen mit mehr als neun Besuchen. Hier könnte eine Stagnation vermutet werden.

Beim Index „Einstellungen“ lassen sich lediglich signifikante Gruppenunterschiede (Hochberg) zwischen den Befragten mit ein bis zwei Fortbildungsbesu-

19 Die schwankenden Stichprobengrößen in dieser und den folgenden Tabellen sind auf Missings bei den einzelnen eingeflossenen Items zurückzuführen. 
chen und denen mit sieben bis neun Fortbildungsbesuchen (.003) identifizieren. Da bei diesem Index der Cronbach-Alpha-Wert gering ausfiel, sind die Signifikanzwerte kaum interpretierbar.

Tabelle 24. Einfluss der Fortbildungsanzahl auf die Indizes "Professionswissen“, "Motivation“ und „Einstellungen“

\begin{tabular}{lcccccc}
\hline $\begin{array}{l}\text { Anzahl besuchter } \\
\text { Fortbildungen }\end{array}$ & $\begin{array}{c}0-2 \\
(\mathrm{n}=79-113)\end{array}$ & $\begin{array}{c}3-6 \\
(\mathrm{n}=95-140)\end{array}$ & $\begin{array}{c}7-9 \\
(\mathrm{n}=36-43)\end{array}$ & $\begin{array}{c}>9 \\
(\mathrm{n}=32-40)\end{array}$ & $\mathrm{F}$ & Sig. \\
\hline $\begin{array}{l}\text { Index „Professions- } \\
\text { wissen“ }\end{array}$ & 122.25 & 124.16 & 130.08 & 132.93 & $7.5^{*}$ & .000 \\
\hline Index „Motivation“ & 39.23 & 42.07 & 48.00 & 46.82 & $27.0 *$ & .000 \\
\hline $\begin{array}{l}\text { Index „Einstellun- } \\
\text { gen“ }\end{array}$ & 9.68 & 9.98 & 10.65 & 10.35 & $5.0^{*}$ & .002 \\
\hline
\end{tabular}

einfaktorielle Varianzanalyse, ${ }^{\star} p<.05$, zweiseitig

\section{Zusammenfassung FF1}

Vergleichbar mit den Ergebnissen bei den Einzelauswertungen (siehe Abschnitt A 8.1.2) steigt bei der Auswertung auf der Basis der Indizes das Professionswissen der Befragten nach eigener Angabe nicht mit der Berufserfahrung, sondern mit der Anzahl der besuchten Fortbildungen der Initiative „Haus der kleinen Forscher“ kontinuierlich an. Die Lehrkräfte schätzen ihr Professionswissen nur direkt nach der Ausbildung signifikant besser ein als die pädagogischen Fachkräfte. Diesen „Rückstand“ holen die pädagogischen Fachkräfte auf. Sie schätzten sich nach der letzten Fortbildung deskriptiv sogar etwas besser als die Lehrkräfte ein, der Unterschied erreicht aber nicht Signifikanzniveau.

Die Werte des Index „Motivation“ steigen sowohl mit der Berufserfahrung als auch mit der Anzahl der besuchten Fortbildungen an; dies zeigte sich ähnlich in den Einzelauswertungen.

Mit dem Index „Einstellungen“ konnte mit Einschränkungen eine positive Entwicklung nur mit der Variable Anzahl besuchter Fortbildungen gemessen werden; das zeigte sich bereits in den Einzelauswertungen.

\subsubsection{FF2 Gibt es verschiedene Phasen, Ereignisse oder typische Abläufe im Professionalisierungsprozess der Fach- und Lehrkräfte?}

Zieht man die im vorherigen Kapitel berichteten Ergebnisse heran, erklärt die Anzahl der besuchten Fortbildungen am besten den selbsteingeschätzten Zuwachs an Professionswissen im Forschen mit Kindern zu MINT-Themen. Damit können als „Ereignis“ im Professionalisierungsprozess die Besuche der Fortbildungen der Initiative „Haus der kleinen Forscher“ betrachtet werden. Mit Bezug auf die vorge- 
fundenen Daten lässt sich der Verlauf am ehesten als stetig beschreiben - auch in den Interviews wurde nicht von einem sprunghaften Kompetenzzuwachs aufgrund eines Einzelereignisses berichtet.

In den Interviews haben sich die Fach- und Lehrkräfte in ganz unterschiedlicher Weise auf die Fortbildungen bezogen. Eine Fachkraft aus Sachsen berichtete, dass die Fortbildung für sie ein Auslöser für eigenes Interesse an und ihre Motivation für MINT-Themen war:

„Ich bin halt hingegangen, weil ich dachte, das muss ich halt machen. Hab“dann aber schnell gemerkt, dass es schöne Weiterbildungen sind, die sehr praktisch ausgerichtet sind, und hab das dann halt so für mich entdeckt. Ich bin dann auch immer weiter hingegangen, weil es gibt ja so viele Themen. “

Als prägend wurde das selbstständige Forschen und Entdecken in den Fortbildungen empfunden; dadurch sei Verständnis ausgelöst worden. Eine Fachkraft aus Nordrhein-Westfalen erklärte: „Also ich kann nicht sagen, ich hab“das und das gelernt, aber die Sachen, die man selber früher nicht verstanden hat, durch Experimente erstmal klar werden, verstehen. “ Zwei weitere Fachkräfte bezeichneten das Verstehen, was bei ihnen durch das Forschen und Entdecken ausgelöst wurde, als „Aha-Moment“. In welcher Weise das fachdidaktische Wissen durch die Fortbildungen nach Angaben der Fach- und Lehrkräfte gefördert wurde, wurde bereits oben beschrieben.

Wie sich das eigene professionelle Verhalten durch die Fortbildungen verändert, beschrieb eine Lehrkraft aus Nordrhein-Westfalen:

„Für mich waren das so ein paar Verhaltensweisen für mich als Durchführer. Damals in meiner Lernwerkstatt lief das alles ein bisschen anders ab. Ich hab' in der Schule gesehen, dass es sinnvoller ist, das so zu machen als so, wie ich das früher gemacht habe. Sowas wie die Kinder erstmal komplett machen zu lassen, mehr Freiraum geben. In der Lernwerkstatt gab es immer mehr Vorgaben noch.“

Auch ein Erzieher aus Nordrhein-Westfalen gab an, dass es ihm anfangs schwerfiel, die Kinder die Lösung selbstständig entdecken zu lassen: „[...] ohne konkrete Antworten gleich zu geben [...] und das habe ich mir dann angewöhnt. Das so zu machen immer.“

Es wurde über alle 15 Interviews hinweg insgesamt fünfundzwanzigmal benannt, dass das Forschen mit Kindern nach den Fortbildungen leichter fallen würde, weil diese das professionelle Handeln und die didaktischen Kenntnisse fördern würden und konkrete Anregungen zum Forschen gäben. 
Ebenso scheint die Fähigkeit, den „Forschungskreis“ (vgl. Marquardt-Mau, 2004, 2011; Rank et al., 2018; Stiftung Haus der kleinen Forscher, 2013) als Werkzeug für das Forschen mit Kindern zu nutzen, ein Entwicklungsprozess zu sein. So berichtete eine pädagogische Fachkraft aus Baden-Württemberg, die bereits zwölf Fortbildungen besucht hat: „[...] je mehr Fortbildungen wir besuchen, je mehr wir damit arbeiten, desto einfacher wird das! “

Danach gefragt, was sich konkret an den eigenen Forschungsaktivitäten durch die Teilnahme an den Fortbildungen der Initiative „Haus der kleinen Forscher“ verändert habe, wurden im Online-Fragebogen verschiedene Dinge genannt. Demnach würde häufiger, selbstbewusster und auch zu vielfältigeren Themen geforscht, und die Ideen aus der Fortbildung mit Kolleginnen und Kollegen würden geteilt. Interessant sind einige Unterschiede zwischen den Beurteilungen der pädagogischen Fach- und Lehrkräfte. Deutlich mehr pädagogische Fachkräfte forschen nach den Fortbildungen häufiger mit den Kindern und richten regelmäßige Forscherzeiten sowie Forschungsateliers ein (siehe Abbildung 16). Dies könnte daran liegen, dass Lehrkräfte in dieser Hinsicht durch die andere Rahmung und Taktung in der Schule weniger häufige Umsetzungsmöglichkeiten haben.

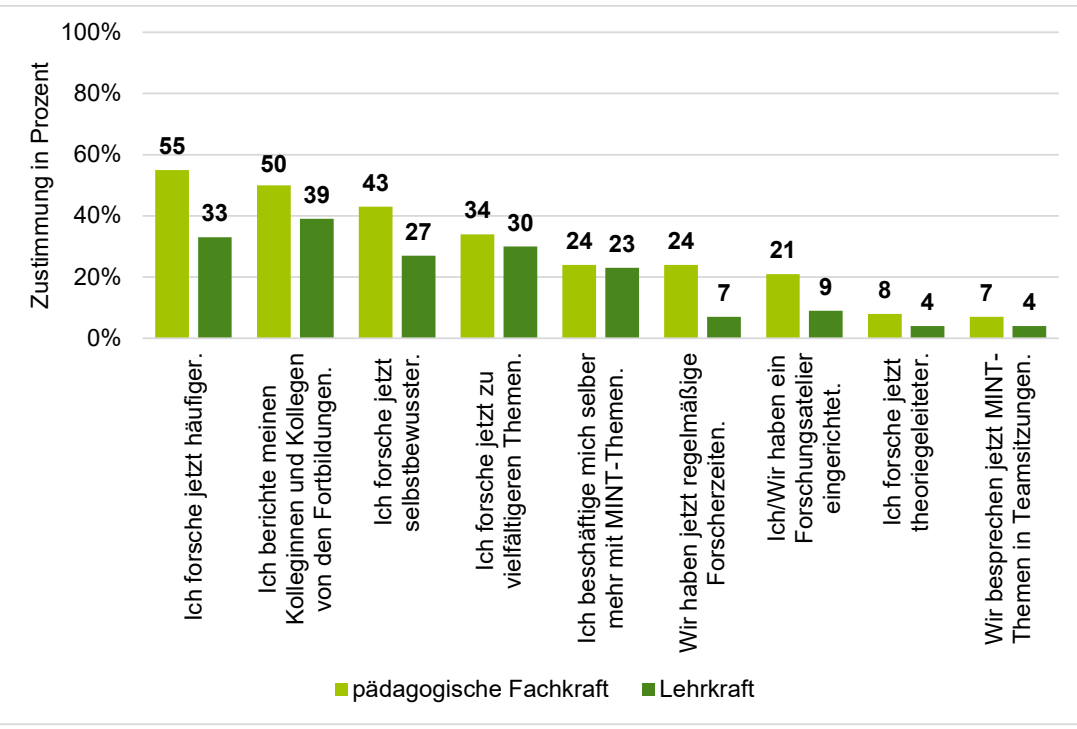

Abbildung 16. Konkrete Veränderungen nach den Fortbildungen der Initiative „Haus der kleinen Forscher" (Mehrfachantworten sind zulässig, Item 6.21)

Die Motivation, mit Kindern zu forschen, steigt dagegen direkt nach dem Besuch einer Fortbildung nur bei etwa einem Drittel an: 50 Prozent der pädagogischen Fachkräfte sowie 44 Prozent der Lehrkräfte geben im Online-Fragebogen an, dass sie immer gleich motiviert seien zu forschen (Item 8.24). 32 Prozent der Lehrkräf- 
te und 20 Prozent der pädagogischen Fachkräfte gaben an, dass ihre Motivation unabhängig vom Besuch der Fortbildungen schwanke, während 24 Prozent der Lehrkräfte und 30 Prozent der pädagogischen Fachkräfte immer direkt nach der letzten Fortbildung besonders motiviert seien. Auf die Frage, wie sich durch die Fortbildungen die Einstellungen zum Forschen mit Kindern verändert haben, äußerten die Interviewten, dass sie die „Angst“ vor Naturwissenschaften (acht Aussagen) nehmen und einen neuen Blick auf MINT-Themen (drei Aussagen) vermitteln würden.

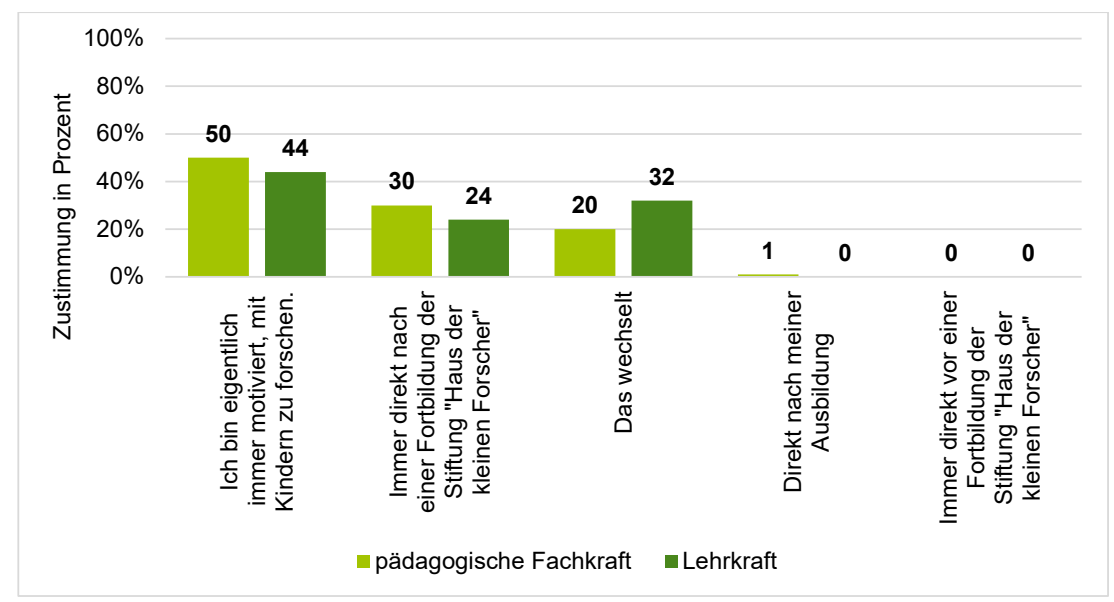

Abbildung 17. Zeitpunkt besonders hoher Motivation, mit Kindern zu experimentieren (Item 8.24)

\section{Zusammenfassung FF2}

Die Fortbildungen der Initiative „Haus der kleinen Forscher“ können als zentrale Ereignisse im Entwicklungsverlauf in Bezug auf frühe MINT-Bildung bezeichnet werden, weil durch sie - nach Angaben der Teilnehmerinnen und Teilnehmer das Professionswissen und die Motivation der Fach- und Lehrkräfte zum Forschen mit Kindern nahezu kontinuierlich gefördert werden. 


\section{Diskussion der Ergebnisse}

In diesem Kapitel werden die Ergebnisse der Studie zusammenfassend interpretiert hinsichtlich der Frage, welche Entwicklungsverläufe die befragten Fach- und Lehrkräfte in Bezug auf ihre Professionalisierung im Bereich der MINT-Bildung selbst einschätzten.

In Anlehnung an die COAKTIV-Studie von Kunter et al. (2011) zur professionellen Kompetenz von Lehrkräften wurden in dieser Studie verschiedene Facetten der professionellen Kompetenz von Fach- und Lehrkräften im MINT-Bereich erfasst. Dazu wurden die Teilnehmerinnen und Teilnehmer an Fortbildungen der Initiative „Haus der kleinen Forscher“ nach ihrer Einschätzung bezüglich ihres Professionswissens, ihrer Motivation und ihren Einstellungen gefragt. Verläufe der Kompetenzentwicklung wurden über den Vergleich der Aussagen von erfahrenen und weniger erfahrenen Fach- und Lehrkräften nachgezeichnet. Die Erfahrung wurde dafür hinsichtlich der Anzahl an Berufsjahren, der Anzahl besuchter Fortbildungen und einem möglichen MINT-Hintergrund operationalisiert. Weiter wurde die Entwicklung durch eine retrospektive Einschätzung der Fach- und Lehrkräfte hinsichtlich der Veränderung ihres Professionswissens nachgezeichnet.

\subsection{Professionswissen: Stand und Entwicklung}

Das Professionswissen wurde durch die Bereiche fachdidaktisches Wissen sowie professionelle Verhaltensoptionen abgefragt und über Items zur Nutzung des „Forschungskreises“ (vgl. Marquardt-Mau, 2004, 2011; Rank et al., 2018; Stiftung Haus der kleinen Forscher, 2013), zum Verhalten in Beispielsituationen sowie zur Fähigkeit, verschiedene Phänomene kindgerecht erklären zu können, operationalisiert. Der Schwerpunkt lag auf dem fachdidaktischen Wissen; inhaltlich stehen die Items in einem engen Zusammenhang zum Fortbildungskonzept der Stiftung „Haus der kleinen Forscher“. Insgesamt schätzten die Befragten hierbei ihre eigene Kompetenz relativ hoch ein, besonders auffällig war dies bei den pädagogischen Fachkräften. Dieser Befund steht im Gegensatz zu Studien, die das Professionswissen im MINT-Bereich bei Fach- und Lehrkräften objektiv erfasst haben und insgesamt als eher niedrig einschätzen (vgl. Appleton, 2003; Dunekacke, 2015; Garbett, 2003)2015; Garbett, 2003. Offenbar stimmen selbsteingeschätzte und objektiv gemessene Fachkompetenzen in der Höhe nicht überein (Müllerbuchhof \& Zehrt, 2006). In der vorliegenden Studie handelt es sich um Selbsteinschätzungen der Fach- und Lehrkräfte, deren Antworten auch durch sozial erwünschtes Verhalten beeinflusst sein können. 
Befragt nach der Veränderung dieser Kompetenzfacette fanden sich in der retrospektiven Einschätzung des eigenen Professionswissens zu verschiedenen Zeitpunkten signifikante Unterschiede und damit Anhaltspunkte, die auf einen Entwicklungsprozess hindeuten.

Um einen Entwicklungsprozess im Professionswissen abzubilden, wurden die Merkmale Berufserfahrung, MINT-Hintergrund und Anzahl besuchter Fortbildungen herangezogen. Die Anzahl der besuchten Fortbildungen erwies sich als relevant für den Kompetenzzuwachs; dagegen ließen sich in den Antworten der befragten Fach- und Lehrkräfte Kompetenzzuwächse weder durch den beruflichen Hintergrund noch die Berufserfahrung erklären. Obwohl es sich auch hier um Selbsteinschätzungen handelt, deckt sich das Ergebnis mit Studien, die keinen Kompetenzzuwachs allein durch die Berufsjahre feststellen (Lipowsky \& Rzejak, 2012; Nitsche et al., 2013; Steffensky et al., 2018; Brunner et al., 2006), sondern den Zusammenhang mit besuchten Fortbildungen herstellen (Richter et al., 2018; Lipowsky \& Rzejak, 2012; Nitsche et al., 2013; Steffensky et al., 2018). In der Studie EASI Science konnte hingegen gezeigt werden, dass die tatsächliche Sachund Handlungskompetenz der Fach- und Lehrkräfte durchaus mit den Berufsjahren steigt (vgl. Steffensky et al., 2018).

Legt man die Anzahl der besuchten Fortbildungen den Analysen zugrunde, gaben die Befragten an, dass mit zunehmendem Fortbildungsbesuch ihr selbstwahrgenommenes Professionswissen steigt. Das zeigte sich sowohl im direkten Vergleich zwischen erfahrenen und unerfahrenen Fach- und Lehrkräften als auch in der retrospektiven Einschätzung durch die Befragten selbst. Damit werden die Fortbildungen der Initiative „Haus der kleinen Forscher“ als zentrale Lerngelegenheiten wahrgenommen (Kunter et al., 2011), die für einen Entwicklungsverlauf verantwortlich gemacht werden.

\subsection{Motivation: Stand und Entwicklung}

Als weitere Facette der professionellen Kompetenz wurde die Motivation, sich mit MINT-Themen zu beschäftigen, erhoben (Kunter et al., 2011). Die Motivation hat einen großen Einfluss darauf, sich im MINT-Bereich fortzubilden und mit Kindern zu forschen (Rost-Roth, 2001). Erwartungsgemäß gaben die Fach- und Lehrkräfte hohe Motivationswerte an, sonst würden sie vermutlich auch keine Fortbildungen der Initiative „Haus der kleinen Forscher“ besuchen und nicht an einer solchen Befragung teilnehmen. Die Motivation wurde im Fragebogen über mehrere Items wie die Anzahl der mit den Kindern durchgeführten Forschungsaktivitäten und Umsetzung von Beispielexperimenten oder zur Häufigkeit des Forschens allgemein abgefragt. Alle Befragten gaben durchweg hohe Zustimmungswerte an, was sicher auch mit der Zusammensetzung der Stichprobe zu erklären ist. Als weiterer 
Bereich wurde unter Motivation die Selbstwirksamkeitserwartung erhoben (siehe Abschnitt A 6.3.2), die ebenfalls bei allen Befragten in einem hohen Bereich lag.

Bedeutsame Unterschiede hinsichtlich der Motivation fanden sich für den MINT-Hintergrund: Sowohl Lehrkräfte als auch Fachkräfte mit einem MINT-Hintergrund gaben an, motivierter zu sein, mit Kindern zu MINT-Themen zu forschen, sich mit den MINT-Themen zu beschäftigen etc. Hinsichtlich der Entwicklungsverläufe gaben die Befragten an, dass sich ihre Motivation bezüglich MINT-Themen mit zunehmender Berufserfahrung und der Anzahl der besuchten Fortbildungen signifikant gesteigert habe. Das bezieht sich sowohl auf die Anzahl der Forschungsaktivitäten mit Kindern als auch auf die Selbstwirksamkeitserwartung. Lediglich bei Personen, die bereits neun und mehr Fortbildungen der Initiative „Haus der kleinen Forscher“ besucht haben, stieg die Motivation nicht mehr weiter an. Diesen Sachverhalt fanden auch Schulz-Hardt, Rott, Meinken und Frey (2001), der einen Anreizverlust und einen zunehmenden Widerwillen gegenüber der wiederholten Ausführung einer spezifischen Handlung kennzeichnet, im Fall der vorliegenden Studie die wiederholte Teilnahme an Fortbildungen. Denkbar wäre außerdem, dass sich die Befragten nach so vielen besuchten Fortbildungen kompetent genug fühlen und keine weitere Fortbildung mehr benötigen.

An Unterschieden zwischen den beiden Professionen hinsichtlich der Selbstwirksamkeitserwartung zeigte sich, dass diese bei den Lehrkräften im Schnitt höher ist als bei den pädagogischen Fachkräften. Dies ist vermutlich darauf zurückzuführen, dass von den Lehrkräften die Mehrheit einen MINT-Hintergrund hat (nur rund 26 Prozent haben keinen MINT-Hintergrund) und die pädagogischen Fachkräfte mehrheitlich keinen (74 Prozent) MINT-Hintergrund aufweisen können. Die pädagogischen Fachkräfte nehmen jedoch häufiger an Fortbildungen teil, sodass hinsichtlich der Motivation im Allgemeinen (Index „Motivation“) keine signifikanten Unterschiede zwischen den Professionen bestehen.

\subsection{Einstellungen: Stand und Entwicklung}

Auch bei den Einstellungen zur Bedeutung von MINT-Bildung bereits für junge Kinder sind hohe Werte bei den Befragten erwartbar: Würde man das Forschen mit Kindern zu MINT-Themen für weniger wichtig erachten, würde man keine Fortbildungen der Initiative „Haus der kleinen Forscher“ besuchen. Entsprechend haben alle Befragten angegeben, dass sie die MINT-Bildung als wichtig oder sehr wichtig einschätzen - hier fanden sich kaum nennenswerte Veränderungen über die Zeit.

Außerdem ist der Befund erwartbar, dass Fach- und Lehrkräfte mit MINT-Hintergrund die Förderung im MINT-Bereich als wichtiger einschätzten als Pädagoginnen und Pädagogen ohne MINT-Hintergrund, vor allem für den Kita-Bereich. Auf Einzelitemebene zeigt sich, dass alle Pädagoginnen und Pädagogen MINT- 
Förderung im Grundschulbereich als wichtiger einschätzen, was vermutlich auf die längere Tradition solcher Themen im Sachunterricht der Grundschule zurück zu führen ist. Die Einstellung gegenüber der Wichtigkeit des Förderbereichs MINT im Kindergarten ist bei pädagogischen Fachkräften sowie Lehrkräften am positivsten, die bis zu zwei Fortbildungen besucht haben, nicht bei denjenigen mit mehr Fortbildungsbesuchen; dieser Unterschied ist signifikant. Für den Grundschulbereich bleibt die Einstellung über die steigende Anzahl von Fortbildungsbesuchen nahezu gleich. Diese Ergebnisse decken sich mit bereits berichteten Annahmen, dass Überzeugungen recht stabil sind (Kunina-Habenicht, Decker \& Kunter, 2016), aber gleichzeitig scheinen Fortbildungen auch das Potenzial für Veränderungen von Einstellungen zu bieten (Kunina-Habenicht, Decker \& Kunter, 2016; Lipowsky \& Rzejak, 2012). Hinweise darauf finden sich in den Ergebnissen aus dem kumulierten Index „Einstellungen“: Durch eine steigende Anzahl an Fortbildungsbesuchen entwickeln sich die Einstellungen gegenüber MINT-Themen signifikant positiv.

\subsection{Fazit}

Insgesamt zeichneten die Befragten ein äußerst positives Bild sowohl ihrer professionellen Kompetenz als auch ihrer Entwicklung, die sie durch die Fortbildungen im Rahmen der Initiative „Haus der kleinen Forscher“ erfahren haben. 50 Prozent der Befragten sahen keinen Bedarf, das Fortbildungsangebot der Initiative „Haus der kleinen Forscher“ zu verändern und sprachen sich dafür aus, das bisherige Format genauso weiterzuführen. Die andere Hälfte formulierte Änderungsvorschläge in eher begrenztem Rahmen, z. B. mehr praktische Tipps zum Forschen mit Kindern zu erhalten oder die unterschiedlichen Altersstufen von Kindern mehr zu berücksichtigen. Die Varianzen im Antwortverhalten waren generell gering, große Unterschiede konnten weder zwischen den Berufsgruppen (Fach- und Lehrkraft) noch zwischen Personengruppen mit unterschiedlichem Erfahrungshintergrund ausgemacht werden. So handelt es sich bei den in Kapitel A 8 und im Folgenden berichteten Unterschieden in den Entwicklungen nur um geringe Ausprägungen. Demzufolge stellt sich die Gruppe der befragten Fach- und Lehrkräfte als recht homogene Gruppe dar, was ihre Einschätzungen bezüglich ihrer professionellen Kompetenz im MINT-Bereich und deren Entwicklung angeht. Unterschiede zeigten sich erst in detaillierten Analysen.

Um die Entwicklungsverläufe von Fach- und Lehrkräften in ihrer professionellen Kompetenz im MINT-Bereich zu beschreiben, erwiesen sich die Teilnahmen an Fortbildungen der Initiative „Haus der kleinen Forscher“ als prägende Lerngelegenheiten. Nach ihrer eigenen Wahrnehmung steigerten sich vor allem das Professionswissen und die Motivation bezüglich MINT-Themen und dem Forschen 
mit Kindern mit zunehmender Anzahl besuchter Fortbildungen. Obwohl es sich um Selbsteinschätzungen handelt, wurden im Rahmen der EASI-Science-Studie (Steffensky et al., 2018) Unterschiede hinsichtlich des Fachwissens, der Motivation und der Einstellungen von pädagogischen Fachkräften abhängig von der Häufigkeit naturwissenschaftlicher Fortbildungen auch mithilfe von Fremdeinschätzungen berichtet. Unterschiede im fachdidaktischen Wissen dagegen konnten dort nicht nur auf die Häufigkeit des Besuchs von Fortbildungen, sondern auch auf einen MINT-Hintergrund zurückgeführt werden (Steffensky et al., 2018). Dieser Zusammenhang zeigte sich in der vorliegenden Studie dagegen nicht, was sicher auf die Selbsteinschätzung zurückzuführen ist.

Als besonders wichtige Rahmenbedingungen für den Erfolg der Fortbildungen für ihre Professionalisierung nannten die Befragten in der Online-Erhebung der vorliegenden Studie das eigene Forschen und Entdecken, die Verknüpfung von Theorie und Praxis in der Fortbildung sowie das regelmäßige und vielfältige Angebot der Fortbildungen. Die Interviewteilnehmerinnen und -teilnehmer betonten vor allem das selbstständige Forschen und Entdecken in den Fortbildungen als Schlüsselereignisse („Aha-Momente“) für das Verstehen. Fortbildungen dienen nach eigener Aussage auch dazu, Hemmnisse und Ängste hinsichtlich des Forschens im MINT-Bereich abzubauen und die Selbstwirksamkeitserwartung zu steigern, insbesondere bei pädagogischen Fachkräften. Die Bedeutung von spezifischem Fachwissen bleibt dabei unklar.

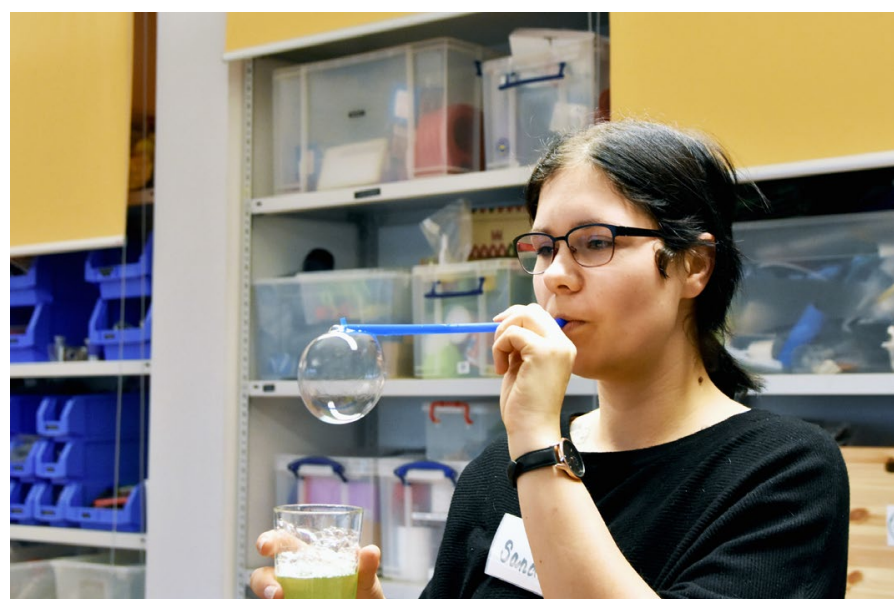




\section{Grenzen der Studie}

Neben den Befunden, die die durchgeführten Interviews und die Online-Befragung ergeben haben, stößt diese eher explorativ angelegte Studie inhaltlich und methodisch an verschiedenen Stellen an ihre Grenzen: Zum einen war es eine besondere Schwierigkeit, aus einer Querschnitterhebung wie dieser Entwicklungsverläufe pädagogischer Fach- und Lehrkräfte abzuleiten. Dazu wäre eine Längsschnittstudie mit verschiedenen Messzeitpunkten geeigneter gewesen, was allerdings aufgrund der kurzen Laufzeit des Projekts nicht möglich war. Der Versuch, sich diesen Verläufen dennoch über Hilfskonstruktionen (retrospektive Einschätzung sowie der Vergleich zwischen erfahrenen und weniger erfahrenen pädagogischen Fach- und Lehrkräften) zu nähern, konnte lediglich einige Dimensionen aufdecken, entlang derer sich unterschiedliche Entwicklungen bei den pädagogischen Fach- und Lehrkräften zeigen.

Obwohl in der Studie zahlreiche Unterschiedsberechnungen durchgeführt und Signifikanzwerte berichtet wurden, weisen diese eher geringe Effektstärken auf. Insgesamt zeigen die Antworten der Befragten nur eine sehr geringe Varianz, sodass Mehrebenenanalysen nicht gerechnet werden konnten, obwohl das angesichts der Anlage der Studie und der Stichprobenmerkmale angebracht gewesen wäre.

Zum anderen basieren alle Aussagen zu Professionswissen, Motivation und Einstellungen auf Selbsteinschätzungen der Befragten und wurden nicht objektiv beobachtet, z. B. in Praxissituationen gemessen. Kucharz et al. (2014) fassen bisherige Forschungsarbeiten mit dem Resultat zusammen, dass die Kompetenzselbsteinschätzung bei pädagogischen Fachkräften gegenüber der Fremdeinschätzung positiver ausfällt. Das Problem dabei besteht einerseits in der Möglichkeit, sich über- beziehungsweise unterschätzen zu können, und zum anderen in den bei Befragungen oft auftretenden Antworten nach sozialer Erwünschtheit. So erstaunen die Ergebnisse, dass die pädagogischen Fachkräfte ihr Professionswissen höher einschätzen als die Lehrkräfte nur auf den ersten Blick: Lehrkräfte haben zwar häufiger einen MINT-Hintergrund, pädagogische Fachkräfte besuchen aber im Schnitt häufiger Fortbildungen, was der ausschlaggebende Faktor für die drei Facetten des Professionswissens zu sein scheint.

Es ist beispielsweise auch nicht möglich, Aussagen zur Qualität des forschenden Lernens der Befragten mit Kindern zu treffen, sondern wir erfahren lediglich, wie diese Qualität subjektiv empfunden wurde. Zudem konnte die Umsetzung der Fortbildungsinhalte in die berufliche Praxis der pädagogischen Fach- und Lehrkräfte nicht direkt untersucht werden. Dazu wären unter Umständen Videoanalysen oder Beobachtungen vor Ort notwendig gewesen. 
Des Weiteren ist es möglich, dass die Stichprobe der Online-Erhebung verzerrt sein könnte. Es liegt die Vermutung nahe, dass nur besonders motivierte Probandinnen und Probanden vertreten sind, die bereit waren, einen solch umfangreichen Fragebogen auszufüllen, und daher ein recht einheitlich positives Bild von den Fortbildungen der Initiative „Haus der kleinen Forscher“ gezeichnet wird. Diese Vermutung wird bestärkt durch den Umstand, dass die Befragten überproportional häufig in einer Einrichtung tätig sind, die als „Haus der kleinen Forscher“ zertifiziert ist (im Vergleich zur Gesamtheit der Einrichtungen, die an Fortbildungen der Initiative teilnehmen) und deshalb eine größere Nähe zu MINTThemen haben.

Hinsichtlich der Anzahl der besuchten Fortbildungen, der Profession und der Einrichtungstypen konnte die Stichprobe die teilnehmenden Fach- und Lehrkräfte an Fortbildungen der Initiative „Haus der kleinen Forscher“ insgesamt in etwa repräsentieren.

Was zusätzlich kritisch hinterfragt werden sollte, ist der Wunsch einiger Befragten nach weniger Theorie und mehr praxisnahen Tipps in den Fortbildungen. Es scheint, als besäße das Fach- und fachdidaktische Wissen zu MINT-Themen für die Befragten eine untergeordnete Rolle gegenüber dem Angebot an schnellen Lösungen für den Alltag. Letztere sollten jedoch eher als Anregungen für die berufliche Praxis gesehen werden, da immer, wenn es um eine angemessene Bildung von Kindern geht, theoretische Kenntnisse zum jeweiligen Thema und seiner Vermittlung unabdingbar sind (Schäfer, 1995). 


\section{Schlussfolgerungen für die Arbeit der Stiftung „Haus der kleinen Forscher“}

Abschließend soll aus den Befunden dieser Studie abgeleitet werden, welche Schlussfolgerungen für die zukünftige Arbeit der Stiftung „Haus der kleinen Forscher“ gezogen werden können.

Insgesamt lassen die Selbstauskünfte der Teilnehmerinnen und Teilnehmer den Schluss zu, dass die Fortbildungen sich positiv auf die Einschätzung des eigenen Professionswissens und der Selbstwirksamkeit auswirken und dass dies unabhängig von der Profession (Vergleich zwischen Fach- und Lehrkräften) geschieht. Objektive Auskünfte über diesen Professionalisierungsprozess können nur weitere Forschungen in der konkreten Praxis geben.

Die Fortbildungen wurden von den befragten pädagogischen Fach- und Lehrkräften als prägend für den Entwicklungsverlauf bezeichnet. Knapp die Hälfte der Teilnehmerinnen und Teilnehmer formuliert Verbesserungsvorschläge.

Gewünscht wurde beispielsweise, dass die Referentinnen und Referenten noch mehr konkrete Tipps zum Forschen mit den Kindern geben sollten. Da vor allem die leichte Umsetzbarkeit der in der Fortbildung vor Ort ausprobierten Forschungsbeispiele und die Anregungen für zuhause geschätzt wurden, verwundert es nicht, dass bei Optimierungsmaßnahmen gerade solche genannt wurden, welche die Übertragbarkeit der Inhalte aus den Fortbildungen in den eigenen beruflichen Alltag tangieren. Es wäre also ratsam, stets darauf zu achten, dass das, was in einer Fortbildung angeboten wird, auch möglichst einfach mit Kindern angewendet werden kann. Das bedeutet etwa, dass auf Materialien zurückgegriffen werden sollte, die ungefährlich und in den Einrichtungen vorhanden sind.

Etwa 40 Prozent der Fachkräfte und knapp 30 Prozent der Lehrkräfte gaben an, dass das Eingehen auf Kinder unterschiedlicher Altersstufen stärker thematisiert werden sollte. Betrachtet man zudem die Hinweise aus der offenen Frage zu weiteren Wünschen an die Stiftung „Haus der kleinen Forscher“, finden sich auch Äußerungen, dass die Fortbildungen zu sehr an Kindern im Kita-Alter und zu wenig auf Grundschulkinder ausgerichtet seien. Empfehlenswert wäre daher eine Überprüfung der Inhalte mit Blick auf altersgemischte Gruppen einerseits sowie einzelne Altersstufen andererseits. Grundschullehrkräfte gaben zudem häufiger an, dass sie sich andere Themen wünschen würden, benannten aber nicht, welche das sind.

Die Rolle der Lernbegleitung, die die Teilnehmerinnen und Teilnehmer der Fortbildungen in ihren Berufen einnehmen, ist ein zentraler Aspekt, wenn es darum geht, Kinder an MINT-Themen heranzuführen. Einige der Befragten wünschten sich hier eine stärkere Fokussierung auf didaktische Aspekte sowie das pädagogi- 
sche Selbstverständnis als Fach- beziehungsweise Lehrkraft. Lehrkräfte wünschten sich auch etwas häufiger eine theoretische Fundierung.

Das Themenangebot der Stiftung „Haus der kleinen Forscher“ wird von der Mehrheit der Befragten als vielseitig und abwechslungsreich bezeichnet. Dennoch sagen knapp ein Fünftel, es wäre ratsam, zukünftige Fortbildungen noch mehr an der Lebenswelt der Kinder auszurichten. Vor dem Hintergrund der MINT-Themen als Fokus der Stiftung „Haus der kleinen Forscher“ könnte man also weiter überlegen, an welchen Stellen es Berührungspunkte gibt mit klassischen MINT-Fragestellungen einerseits und dem Alltag der Kinder andererseits.

Die Austauschphasen in den Fortbildungen sind ein weiterer Aspekt, der wiederholt von den Befragten positiv erlebt wurde. Vor allem die Fachkräfte nutzen in den Fortbildungen die Chance, von Erfahrungen ihrer Kolleginnen und Kollegen zu lernen und Feedback zu ihren eigenen Standpunkten und Methoden zu erhalten. Zukünftige Fortbildungen sollten daher stets im Blick haben, dass genügend Zeit für Austausch zur Verfügung steht, um mit den anderen Teilnehmerinnen und Teilnehmern ins Gespräch zu kommen. Lehrkräfte nennen diesen Punkt seltener; es könnte sein, dass er ihnen weniger wichtig scheint beziehungsweise der Austausch schon in ausreichendem Maße zur Verfügung steht.

Die Verknüpfung von Theorie und Praxis ist zwar eines der Aushängeschilder der Stiftung „Haus der kleinen Forscher“, jedoch haben die Interviews und Befragungen ergeben, dass es weniger der theoretische Input als vielmehr die Beispielexperimente sind, die die Teilnehmerinnen und Teilnehmer begeistern. Frei nach dem Motto „man muss nicht alles erklären können“ wurde wiederholt angedeutet, dass zugunsten von mehr Praxisphasen eher auf Theorie verzichtet werden sollte. Gegebenenfalls, so wurde betont, könne man sich nach einer Fortbildung in den Handreichungen und Literaturempfehlungen für zuhause noch einmal genauer anschauen, warum was wie geschieht. Für zukünftige Fortbildungen bedeutet das, auf die Bedeutung des Fachwissens hinzuweisen und den theoretischen Input mit den Praxiserfahrungen gezielter zu verzahnen.

Insgesamt gesehen wird das Fortbildungsformat der Stiftung „Haus der kleinen Forscher“ geschätzt. Der „Forschungskreis“ (vgl. Marquardt-Mau, 2004, 2011; Rank et al., 2018; Stiftung Haus der kleinen Forscher, 2013) als Hilfestellung für das Forschen mit Kindern zu MINT-Themen sei zwar nicht immer einfach umzusetzen, biete aber eine Orientierungshilfe und Handlungsempfehlungen, auf die sich die pädagogischen Fach- und Lehrkräfte beziehen können, wenn sie mit Kindern entdecken und forschen. Es ist daher zu empfehlen, ihn auch zukünftig in das Fortbildungsprogramm zu integrieren.

Bezüglich der Entwicklungsverläufe der Befragten lässt sich feststellen, dass sowohl ihr Professionswissen als auch die Motivation zum Forschen mit Kindern mit der Anzahl der besuchten Fortbildungen in der Initiative „Haus der kleinen 
Forscher" anwächst beziehungsweise sich zum Positiven weiterentwickelt. Viele der Befragten äußerten zudem, dass sie gerne an mehr als zwei Fortbildungen pro Jahr teilnehmen würden, was aber meist aus organisatorischen Gründen in ihrer eigenen Einrichtung nicht möglich ist, wie Aussagen in den Interviews zeigten.

Gewünscht wurde vor diesem Hintergrund, den Angebotsturnus der Initiative „Haus der kleinen Forscher“ zu erhöhen und zudem auch die regionale Verteilung der Orte, an denen die Fortbildungen stattfinden, zu erweitern. Da die OnlineFormate bislang noch wenig angenommen werden - 87 Prozent aller Befragten haben noch nicht auf das Online-Angebot zugegriffen -, könnte hierbei über eine sinnvolle Verknüpfung mit und Ergänzung zu den bestehenden Fortbildungen nachgedacht werden. Dazu würde sich eine wissenschaftliche Begleitforschung anbieten, um Angebot und Nutzung der Lerngelegenheiten gut aufeinander abzustimmen. 


\section{B Kompetenzentwicklung von Pädagoginnen und Pädagogen im Längsschnitt}

Stiftung Haus der kleinen Forscher

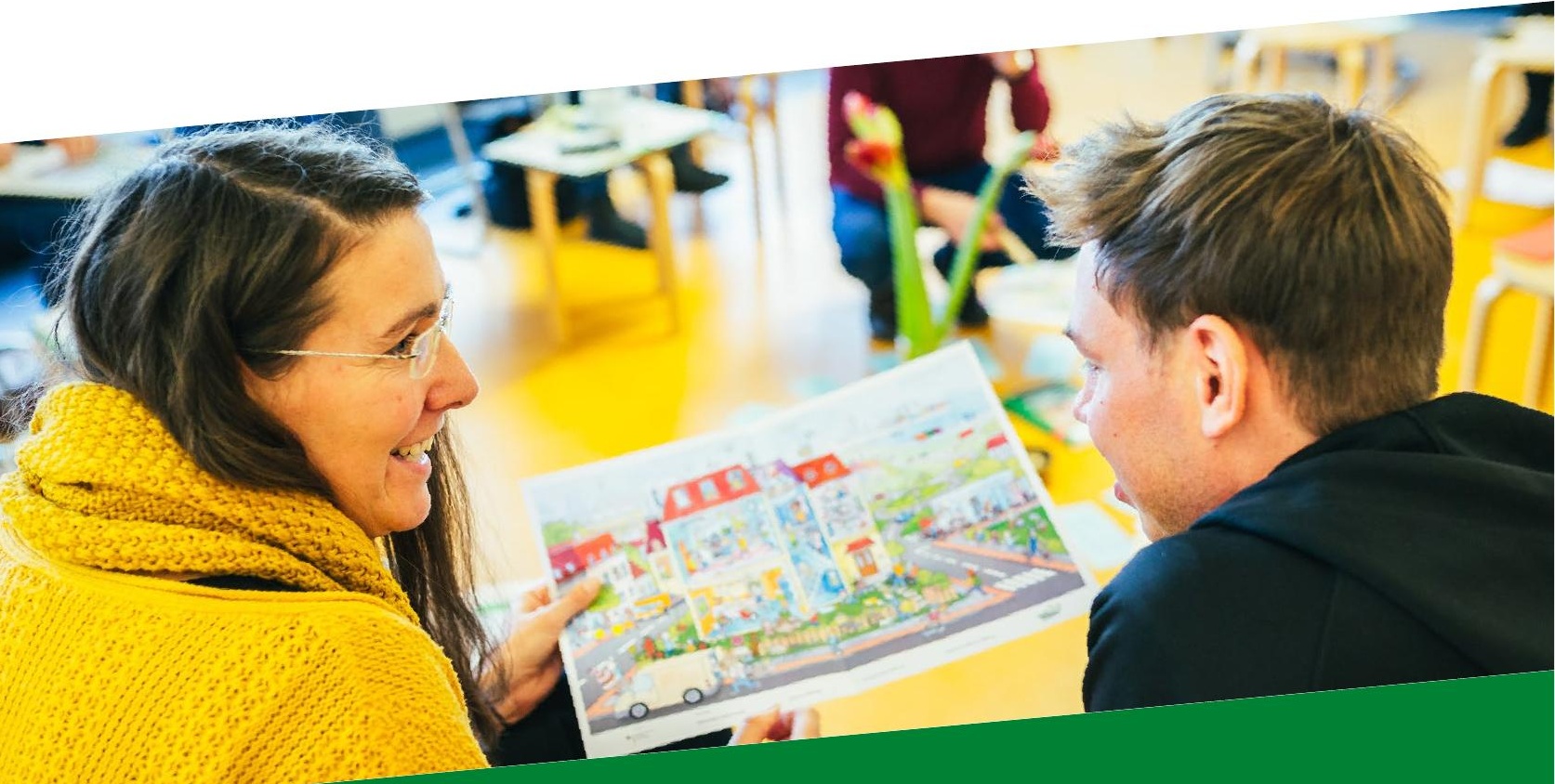

1 Hintergrund der Längsschnittanalysen zu Wirkungen des „Haus der kleinen Forscher"-Fortbildungsangebots

2 Design und Stichprobenbeschreibung der Längsschnittbefragung

3 Ergebnisse

4 Allgemeine Diskussion 


\section{Hintergrund der Längsschnittanalysen zu Wirkungen des „Haus der kleinen Forscher"-Fortbildungsangebots}

\subsection{Inhaltlich-pädagogische Ziele des Fortbildungsprogramms}

Pädagogische Fach- und Lehrkräfte begleiten und unterstützen Kinder in einer sich ständig verändernden Welt. Die Bildungsinitiative „Haus der kleinen Forscher“ verfolgt das Ziel, Pädagoginnen und Pädagogen in ihrer Rolle als Lernbegleitung zu bestärken, und gibt ihnen die Möglichkeit, ihr MINT-Wissen und Wissen zum Bildungskonzept Bildung für nachhaltige Entwicklung (BNE) sowie die dazugehörigen pädagogischen Strategien zu erweitern und zu vertiefen. Das pädagogische Konzept und die Zieldimensionen früher MINT-Bildung und früher Bildung für nachhaltige Entwicklung der Stiftung „Haus der kleinen Forscher“ auf Ebene der pädagogischen Fach- und Lehrkräfte sind grundlegend für jede Fortbildung aus dem Bildungsangebot. Sie beziehen sich auf die Begeisterung für das gemeinsame Entdecken und Forschen mit Kindern, pädagogische Unterstützungsmöglichkeiten, fachdidaktisches Wissen und Handeln, wissenschaftliche Denk- und Arbeitsweisen, Wissen und Einstellungen zu früher MINT-Bildung und früher Bildung für nachhaltige Entwicklung sowie das professionelle Rollen- und Selbstverständnis (vgl. Anders, Hardy, Pauen \& Steffensky, 2013; Benz, Grüßing, Lorenz, Selter \& Wollring, 2017; Bergner et al., 2018; Kosack, Jeretin-Kopf \& Wiesmüller, 2015; Stiftung Haus der kleinen Forscher, 2019).

Als wirkungsorientiert arbeitende Organisation reflektiert die Stiftung ihren Wirkungserfolg in Bezug auf diese Dimensionen fortschreitend durch interne und externe Untersuchungen. Die Entwicklung der Befragungsinstrumente und die Auswertung der Ergebnisse der in 2018 begonnenen Längsschnittbefragung orientieren sich an einem Wirkungsmodell der Kompetenzentwicklung von Pädagoginnen und Pädagogen.

\subsection{Wirkungsmodell der Kompetenzentwicklung}

Um Aussagen über die Wirkungen der Bildungsangebote der Initiative „Haus der kleinen Forscher" treffen zu können, wurde ein Wirkungsmodell der Kompetenzentwicklung von Pädagoginnen und Pädagogen in der frühen MINT-Bildung konzipiert (siehe Abbildung 18). Dieses dient als theoretische Grundlage für die vorlie- 
gende stiftungsinterne Längsschnittbefragung. Bei den folgenden Überlegungen wird von dem Kompetenzbegriff nach Weinert (2001) ausgegangen, nach dem Kompetenzen verstanden werden als „die bei Individuen verfügbaren oder durch sie erlernbaren kognitiven Fähigkeiten und Fertigkeiten, um bestimmte Probleme zu lösen, sowie die damit verbundenen motivationalen, volitionalen und sozialen Bereitschaften und Fähigkeiten, um die Problemlösungen in variablen Situationen erfolgreich und verantwortungsvoll nutzen zu können“(S. 27f.).

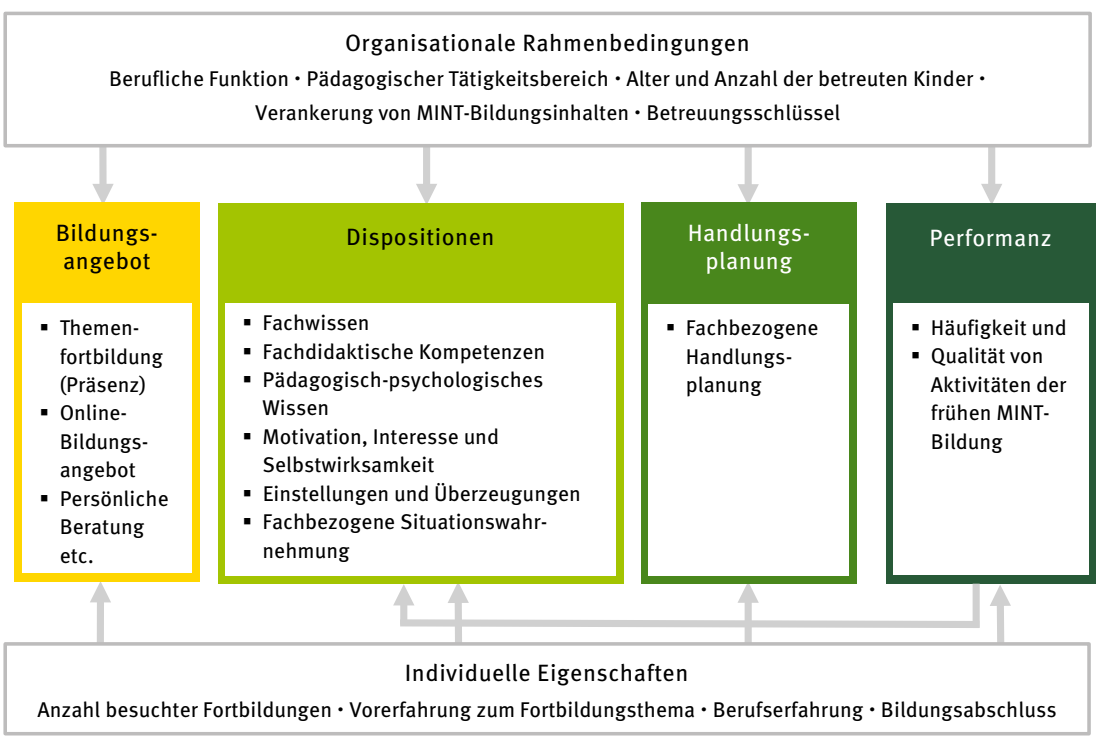

Abbildung 18. Wirkungsmodell der Kompetenzentwicklung von Pädagoginnen und Pädagogen in der frühen MINT-Bildung; in Anlehnung an Fröhlich-Gildhoff, NentwigGesemann und Pietsch (2011, überarbeitete Fassung von 2013; zitiert nach FröhlichGildhoff, 2014)

Das entwickelte Wirkungsmodell orientiert sich am Kompetenzmodell von Fröhlich-Gildhoff, Nentwig-Gesemann und Pietsch (2011). Es handelt sich um ein sogenanntes Prozessmodell, welches ausgehend von einer angestrebten pädagogischen Situation (Bereich Performanz) notwendige Komponenten (Voraussetzungen) als Handlungsanforderungen identifiziert. Diesem prozessorientierten Modell liegt bereits das Strukturmodell von Baumert und Kunter (2011) zugrunde, welches situationsunabhängig wichtige Kompetenzen abbildet und beschreibt. Das Prozessmodell erweitert das Strukturmodell von Baumert und Kunter um situationsspezifische Fertigkeiten und die konkrete Umsetzung von Aktivitäten im pädagogischen Alltag.

Bildungsangebote zielen demnach auf die Veränderung beziehungsweise Erweiterung von Dispositionen der Pädagoginnen und Pädagogen wie Wissen, Mo- 
tivation, Fertigkeiten, Denk- und Wahrnehmungsprozesse sowie Überzeugungen und letztendlich auf Verhaltensänderungen ab (vgl. Fröhlich-Gildhoff, NentwigGesemann \& Pietsch, 2011). Mit dem Ziel der Verhaltensänderung werden in dem Wirkungsmodell situationsunabhängige Kompetenzen aus dem Bereich der Dispositionen um situationsspezifische Fertigkeiten (Bereich Handlungsplanung) ergänzt. Der Gesamtprozess der Kompetenzentwicklung auf den unterschiedlichen verhaltensrelevanten Dimensionen hängt mit individuellen Eigenschaften (z. B. Anzahl besuchter Fortbildungen und Berufserfahrung) sowie organisationalen Rahmenbedingungen (z. B. berufliche Funktion und Betreuungsschlüssel) zusammen (vgl. Fröhlich-Gildhoff, Nentwig-Gesemann \& Pietsch, 2011).

Die einzelnen Komponenten in den Bereichen Dispositionen, Handlungsplanung und Performanz sind in unterschiedlichem Ausmaß durch die Nutzung von Fortbildungsangeboten veränderbar. So sind Entwicklungen in den Komponenten Fachkompetenzen (prozessbezogene und inhaltsbezogene Kompetenzen) oder fachdidaktische Kompetenzen eher und in stärkerem Ausmaß zu erwarten als beispielsweise die Veränderung von Einstellungen und Handlungsroutinen. Es ist davon auszugehen, dass Einstellungen und Orientierungen (sogenannte „beliefs“) relativ stabile Konstrukte darstellen (Anders, 2012; Fröhlich-Gildhoff, Weltzien, Kirstein, Pietsch, Rauh, 2014; Tietze, 1998).

Das dargestellte prozesshafte Wirkungsmodell der Kompetenzentwicklung dient als analytische Grundlage der Längsschnittbefragung und -auswertungen und ist als „lernendes Modell“ gedacht. Dies bedeutet, dass die dem Modell zugrunde gelegten Wirkungsannahmen mit den bis dato sowie den in Folgebefragungen gewonnenen empirischen Erkenntnissen überprüft und darauf aufbauend ggf. angepasst beziehungsweise erweitert werden können.

Das Wirkungsmodell der Kompetenzentwicklung sowie das Befragungsdesign und die leitenden Fragestellungen der Untersuchung wurden in 2018 und 2019 mit dem Wissenschaftlichen Beirat der Stiftung „Haus der kleinen Forscher“ diskutiert. Der Wissenschaftliche Beirat hat insbesondere hinsichtlich der Analysen und der Interpretation der vorliegenden Daten wertvolle Hinweise und Vorschläge beigesteuert. So wurde beispielsweise aufgrund der dezidierten Empfehlungen des Wissenschaftlichen Beirats eine Dropout-Analyse durchgeführt (siehe Abschnitt B 2.1) und bei der Ergebnisinterpretation berücksichtigt.

\subsection{Bisherige Wirkungsanalysen zum Fortbildungsprogramm}

Im Monitoring-Bericht 2016/2017 der Stiftung wurden Wirkungszusammenhänge der Bildungsinitiative analysiert. Gruppenvergleiche lieferten Hinweise darauf, 
dass pädagogische Fach- und Lehrkräfte umso mehr von den „Haus der kleinen Forscher“-Fortbildungen profitieren, je kontinuierlicher sie daran teilnehmen. Es konnte gezeigt werden, dass die Anzahl der Fortbildungsbesuche für eine Wissenserweiterung, Veränderungen in der Selbstwirksamkeitserwartung sowie eine gesteigerte Häufigkeit und eine verbesserte Qualität des gemeinsamen Forschens mit Kindern relevant sind (vgl. Stiftung Haus der kleinen Forscher, 2017).

Diese Ergebnisse stehen in Einklang mit jenen aus externen Untersuchungen zur Bildungsinitiative wie dem Forschungsprojekt Early Steps Into Science (EASI Science): Pädagogische Fachkräfte mit naturwissenschaftlichen Fortbildungen verfügen über höheres naturwissenschaftsbezogenes Fachwissen und mehr fachdidaktische Kenntnisse als eine Vergleichsgruppe ohne solche Fortbildungen. Zudem sind sie motivierter und interessierter an naturwissenschaftlicher Bildung (vgl. Steffensky et al., 2018). Weitere Untersuchungen zum Fortbildungsprogramm finden sich in früheren Monitoring-Berichten der Stiftung (2009-2015) (vgl. Stiftung Haus der kleinen Forscher, 2009, 2010, 2011, 2012, 2013, 2014, 2015). Seit Herbst 2018 führt die Stiftung eine langfristig angelegte Längsschnittbefragung von Pädagoginnen und Pädagogen durch, um die Wirkungen des Fortbildungsprogramms auf die Kompetenzen und Einstellungen zu früher MINT-Bildung und früher Bildung für nachhaltige Entwicklung sowie die Umsetzung der Fortbildungsinhalte in der pädagogischen Praxis zu erheben. Durch diese Längsschnittbefragung kann die professionelle Entwicklung der Pädagoginnen und Pädagogen nach der Teilnahme an „Haus der kleinen Forscher“-Fortbildungsangeboten nachgezeichnet werden (siehe Kapitel B 2).

In Analysen bei über 1.500 pädagogischen Fach-, Lehr- und Leitungskräften zu den ersten zwei Messzeitpunkten der Längsschnittbefragung (vor und direkt nach dem Besuch einer Präsenzfortbildung) zeigten sich Wirkungen auf den selbstberichteten Lernerfolg unmittelbar nach dem Fortbildungsbesuch (vgl. Monitoring-Bericht 2018/2019; Stiftung Haus der kleinen Forscher, 2020). Besonders hinsichtlich der Selbstsicherheit bei der Umsetzung des Fortbildungsthemas und des Wissens in Bezug auf das Entdecken und Forschen mit Kindern schätzen sich die Pädagoginnen und Pädagogen nach dem Fortbildungsbesuch deutlich besser ein. Motivation und Selbstwirksamkeitserwartung in Bezug auf das Entdecken und Forschen mit Kindern werden bereits vor der Teilnahme an der Präsenzfortbildung hoch eingeschätzt und steigen weiter. Gruppenvergleiche lieferten Hinweise darauf, wie die „Haus der kleinen Forscher“-Fortbildungen Wirkungen auf den Lernerfolg entfalten: Die Pädagoginnen und Pädagogen, die zuvor keine Fortbildung besucht haben, weisen den höchsten Zuwachs in den untersuchten Zielbereichen auf. Diese Fortbildungsneulinge beim „Haus der kleinen Forscher“Programm profitieren also kurzfristig stärker von dem Besuch einer einzelnen Präsenzfortbildung als Vergleichsgruppen mit mehr besuchten „Haus der kleinen 
Forscher"-Fortbildungen. Zugleich schätzen die Pädagoginnen und Pädagogen mit vielen besuchten Präsenzfortbildungen bereits vor einer weiteren Fortbildung ihr Wissen, ihre Motivation und ihre Selbstwirksamkeitserwartung umso höher ein, je mehr „Haus der kleinen Forscher“-Präsenzfortbildungen sie zuvor besucht haben. Dieses höhere Ausgangsniveau der weiter fortgeschrittenen Programmteilnehmenden weist auf mögliche längerfristige Wirkungen der Bildungsangebote des „Hauses der kleinen Forscher“ auf den Lernerfolg der Pädagoginnen und Pädagogen hin.

\subsection{Erkenntnisinteresse der vorliegenden Analysen}

In der vorliegenden Untersuchung zur Längsschnittbefragung analysiert die Stiftung die professionelle Kompetenzentwicklung von Pädagoginnen und Pädagogen über drei Messzeitpunkte (im Verlauf von insgesamt bis zu fünf Monaten). Neben den Messzeitpunkten vor und direkt nach dem Besuch einer „Haus der kleinen Forscher"-Präsenzfortbildung wurden die Teilnehmenden auch zwei bis fünf Monate nach Teilnahme an der Fortbildung befragt. Dieser dritte Messzeitpunkt liefert wichtige Hinweise darauf, wie sich die Kompetenzentwicklung der Pädagoginnen und Pädagogen durch den Fortbildungsbesuch im mittelfristigen Verlauf gestaltet. In den Analysen wird für den Einfluss von individuellen und organisationalen Voraussetzungen kontrolliert.

Im Folgenden werden diese Fragestellungen untersucht:

1. Welche durchschnittliche kurz- und mittelfristige Kompetenzentwicklung zeigen Pädagoginnen und Pädagogen nach dem Besuch einer „Haus der kleinen Forscher"-Präsenzfortbildung?

2. Wie unterscheiden sich die kurz- und mittelfristigen Wirkungen der Teilnahme an einer Präsenzfortbildung für Personen mit unterschiedlichen individuellen Eigenschaften und organisationalen Rahmenbedingungen?

3. Welche Zusammenhänge zeigen sich zwischen der Fortbildungsanzahl und -frequenz vor dem betrachteten Fortbildungsbesuch und dem Ausgangsniveau verschiedener Kompetenzen? 


\section{Design und Stichprobenbeschreibung der Längsschnittbefragung}

\subsection{Erhebungsdesign}

Zur Beantwortung der oben aufgeführten Forschungsfragen kam in der vorliegenden Studie ein Längsschnittdesign mit drei Messzeitpunkten zur Anwendung. An den ersten beiden Messzeitpunkten der Längsschnittbefragung nahmen zwischen September 2018 und Februar 2019 deutschlandweit 1.559 pädagogische Fach-, Lehr- und Leitungskräfte aus Kitas, Horten und Grundschulen teil. In 167 „Haus der kleinen Forscher“-Präsenzfortbildungen wurden die Teilnehmenden vor (t0) sowie am gleichen Tag direkt nach der Fortbildung (t1) mittels eines Papierfragebogens befragt. Die Erstbefragung erfolgte in insgesamt 17 lokalen Netzwerken der Bildungsinitiative „Haus der kleinen Forscher“ deutschlandweit. Die teilnehmenden lokalen Netzwerke wurden nach einer Vielzahl an Kriterien sorgfältig ausgewählt. Ausschlaggebend für die Auswahl der an der Befragung teilnehmenden Netzwerke waren eine gleichmäßige regionale Verteilung über das gesamte Bundesgebiet, eine hohe Anzahl an durchgeführten Präsenzfortbildungen im geplanten Erhebungszeitraum und ein möglichst hoher Anteil an sogenannten Fortbildungsneulingen, die das erste Mal eine „Haus der kleinen Forscher“-Fortbildung besuchten.

Für die Folgebefragung einige Monate nach dem Fortbildungsbesuch (t2) wurden die Pädagoginnen und Pädagogen per E-Mail kontaktiert (siehe Abbildung 19). Hierbei betrug der Rücklauf rund 29 Prozent. In einer Dropout-Analyse wurde untersucht, ob es sich bei denjenigen, die nicht an der Folgebefragung teilgenommen haben, um zufällige oder systematische Ausfälle handelt. Die Ergebnisse weisen darauf hin, dass die in den hier dargestellten Analysen betrachtete Stichprobe zum Zeitpunkt t2 im Vergleich zu den nach der Ersterhebung (t0/t1) Ausgeschiedenen leicht motivierter ist und sich geringfügig selbstwirksamer einschätzt, mit Kindern zu entdecken und zu forschen.

Die Themen der „Haus der kleinen Forscher“-Fortbildungen deckten das gesamte Spektrum der Präsenzfortbildungen der Bildungsinitiative zu MINT-Bildung und Bildung für nachhaltige Entwicklung (BNE) ab, das im Zeitraum der Erhebung (September 2018 bis Februar 2019) bundesweit angeboten wurde. Das Angebot an Präsenzfortbildungen der Initiative umfasste im Erhebungszeitraum Fortbildungen mit thematischem Bezug zu den einzelnen MINT-Bereichen (beispielsweise „Zahlen, Zählen, Rechnen“, „Informatik entdecken - mit und ohne Computer“, 
„Forschen mit Wasser“, „Technik - von hier nach da“) sowie zwei Fortbildungen zu Bildung für nachhaltige Entwicklung (BNE).

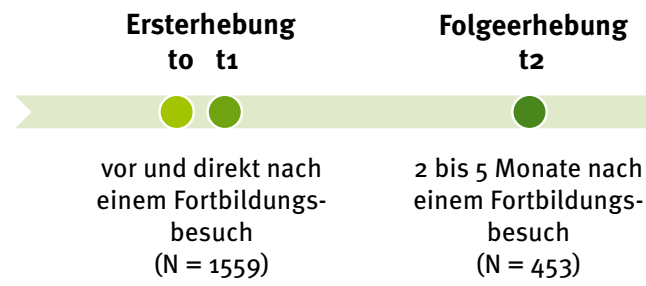

Abbildung 19. Erhebungsdesign der Längsschnittbefragung

Das angewandte längsschnittliche Erhebungsdesign ermöglichte die Bestimmung von durchschnittlichen kurzfristigen (t0 zu t1) und mittelfristigen (t0 zu t2) Effekten der Teilnahme an einer Fortbildung (im Folgenden Fortbildungseffekte genannt) für die Gesamtheit der befragten Pädagoginnen und Pädagogen (Fragestellung 1).

Um ein differenzierteres Verständnis für die Wirkungen des Besuchs der Präsenzfortbildungen zu erhalten, wurden neben den durchschnittlichen Fortbildungseffekten der Befragten zudem Zusammenhänge der Fortbildungseffekte mit individuellen Eigenschaften und organisationalen Rahmenbedingungen der Pädagoginnen und Pädagogen bestimmt (Fragestellung 2). Außerdem wurden die Zusammenhänge der vorherigen Fortbildungsanzahl und -frequenz mit Unterschieden im Kompetenzausgangsniveau der pädagogischen Fach-, Lehr- und Leitungskräfte zum Messzeitpunkt to analysiert, um retrospektiv erste Erkenntnisse in Hinblick auf mögliche längerfristige Teilnahmeeffekte zu gewinnen (Fragestellung 3).

\section{2 Übersicht über die verwendeten Konstrukte und Messinstrumente}

Die Inhalte und Methoden der Präsenzfortbildungen zielen insbesondere darauf ab, die teilnehmenden Pädagoginnen und Pädagogen zu motivieren, mit Kindern zu entdecken und zu forschen, ihre Selbstwirksamkeit dafür zu stärken und ihr dafür nötiges fachdidaktisches Wissen auszubauen (siehe Abschnitt B 1.1; vgl. Stiftung Haus der kleinen Forscher, 2019). Daher lag im Fokus der vorliegenden Untersuchung die Bestimmung von kurz- und mittelfristigen Fortbildungseffekten für verschiedene Kompetenzen.

In Tabelle 25 wird dargestellt, mit welchen Fragebogenitems die Konstrukte fachdidaktisches Wissen, Motivation und Selbstwirksamkeit in Bezug auf das 
Entdecken und Forschen mit Kindern gemessen wurden. Diese Items sind auf der Basis früherer Monitoring-Untersuchungen der Stiftung und im Austausch mit der Forschungsgruppe der externen Studie EASI-Science (Steffensky et al., 2018) entstanden.

Tabelle 25. Erhebung der verwendeten abhängigen Variablen

\begin{tabular}{|c|c|c|c|}
\hline Merkmal & Itemformulierung & MZP & Kodierung \\
\hline $\begin{array}{l}\text { Fachdidaktisches } \\
\text { Wissen in Bezug auf } \\
\text { das Entdecken und } \\
\text { Forschen mit Kindern }\end{array}$ & $\begin{array}{l}\text { Ich weiß, wie ich Anlässe im } \\
\text { Alltag aufgreifen und mit den } \\
\text { Kindern spontan dazu entde- } \\
\text { cken und forschen kann. } \\
\text { Ich weiß, wie ich Forschungs- } \\
\text { projekte gemeinsam mit } \\
\text { Kindern planen und durchfüh- } \\
\text { ren kann. }\end{array}$ & & \\
\hline $\begin{array}{l}\text { Motivation in Bezug } \\
\text { auf das Entdecken und } \\
\text { Forschen mit Kindern }\end{array}$ & $\begin{array}{l}\text { Mir macht es Spaß, Anlässe } \\
\text { im Alltag aufzugreifen und mit } \\
\text { den Kindern dazu spontan zu } \\
\text { entdecken und zu forschen. } \\
\text { Mir macht es Spaß, For- } \\
\text { schungsprojekte gemeinsam } \\
\text { mit Kindern zu planen und } \\
\text { durchzuführen. }\end{array}$ & $\mathrm{t} 0, \mathrm{t} 1, \mathrm{t} 2$ & $\begin{array}{l}\text { Bildung eines Durch- } \\
\text { schnittswerts aus } \\
\text { beiden Items für } \\
\text { jedes Merkmal, } \\
\text { Skala von } \\
1=\text { trifft überhaupt } \\
\text { nicht zu bis } \\
6=\text { trifft voll und } \\
\text { ganz zu }\end{array}$ \\
\hline $\begin{array}{l}\text { Selbstwirksamkeit } \\
\text { in Bezug auf das Ent- } \\
\text { decken und Forschen } \\
\text { mit Kindern }\end{array}$ & $\begin{array}{l}\text { Ich traue mir zu, Anlässe im } \\
\text { Alltag aufzugreifen und mit } \\
\text { den Kindern dazu spontan zu } \\
\text { entdecken und zu forschen. } \\
\text { Ich traue mir zu, Forschungs- } \\
\text { projekte gemeinsam mit } \\
\text { Kindern zu planen und durch- } \\
\text { zuführen. }\end{array}$ & & \\
\hline
\end{tabular}

Anmerkung: t0: vor der Fortbildung; t1: direkt nach der Fortbildung; t2: 2-5 Monate nach der Fortbildung; MZP: Messzeitpunkt.

Im Wirkungsmodell der Kompetenzentwicklung für die Längsschnittbefragung (siehe Abbildung 18) wird angenommen, dass der individuelle Kompetenzentwicklungsprozess der Pädagoginnen und Pädagogen nicht nur mit der Fortbildungsteilnahme, sondern ebenfalls mit individuellen Eigenschaften sowie mit organisationalen Rahmenbedingungen zusammenhängt. Die in die Analyse aufgenommenen Merkmale der befragten Pädagoginnen und Pädagogen ermöglichen die Bestimmung von individuellen Fortbildungseffekten für Personen mit spezifischen Eigenschaften. 


\section{Merkmale auf individueller Ebene}

Auf individueller Ebene wurden in den statistischen Analysen folgende Merkmale berücksichtigt:

- die Anzahl der vor der Ersterhebung insgesamt besuchten „Haus der kleinen Forscher"-Fortbildungen,

- die bisherige Fortbildungsfrequenz (Anzahl zuvor besuchter „Haus der kleinen Forscher"-Fortbildungen pro Jahr),

- die Berufserfahrung sowie

- der höchste Bildungsabschluss.

Wie diese Variablen erhoben wurden, zeigt Tabelle 26. Die Anzahl der zwischen der Erst- und der Folgeerhebung besuchten Fortbildungen floss als Kontrollvariable ein. Dadurch wurde eine Verzerrung der Ergebnisse durch den Umstand ausgeschlossen, dass manche Befragte zwischen den Messzeitpunkten weitere Fortbildungen besucht haben und andere Befragte nicht. Da Alter und Berufserfahrung nach Cohen (1988) sehr hoch $(r=.67, p<.01)$ korrelieren, floss in die Analysen mit der Berufserfahrung die Variable ein, die einen konkreten Bezug zur aktuellen beruflichen Tätigkeit beschreibt.

Tabelle 26. Erhebung der verwendeten unabhängigen Variablen auf individueller Ebene

\begin{tabular}{|c|c|c|}
\hline Merkmal & MZP & Inhalt/Kodierung \\
\hline $\begin{array}{l}\text { Fortbildungsanzahl } \\
\text { vor t0 }\end{array}$ & to & $\begin{array}{l}\text { Anzahl der vor t0 insgesamt besuchten Präsenzfortbildun- } \\
\text { gen bei der Initiative „Haus der kleinen Forscher“ }\end{array}$ \\
\hline $\begin{array}{l}\text { Fortbildungsanzahl } \\
\text { zwischen } \mathrm{t} 1 \text { und } \mathrm{t} 2\end{array}$ & $\mathrm{t} 2$ & $\begin{array}{l}\text { Anzahl der zwischen } \mathrm{t} 1 \text { und } \mathrm{t} 2 \text { besuchten Präsenzfortbildun- } \\
\text { gen bei der Initiative „Haus der kleinen Forscher“ }\end{array}$ \\
\hline Fortbildungsfrequenz & to & $\begin{array}{l}\text { Anzahl besuchter Präsenzfortbildungen bei der Initiative } \\
\text { „Haus der kleinen Forscher“ pro Jahr; Verhältnis aus } \\
\text { — Anzahl der vor t0 besuchten Präsenzfortbildungen bei der } \\
\text { Initiative „Haus der kleinen Forscher“ und } \\
\text { — Teilnahmedauer (Zeitraum zwischen dem Jahr der ersten } \\
\text { Teilnahme an einer Präsenzfortbildung bei der Initiative } \\
\text { „Haus der kleinen Forscher“ und t0) }\end{array}$ \\
\hline Berufserfahrung & $\mathrm{t} 2$ & Dauer in der aktuellen beruflichen Funktion in Jahren \\
\hline $\begin{array}{l}\text { Höchster Bildungs-ab- } \\
\text { schluss }\end{array}$ & $\mathrm{t} 1$ & $\begin{array}{l}1=\text { bisher ohne abgeschlossene (Berufs-)Ausbildung } \\
2=\text { abgeschlossene (Berufs-)Ausbildung } \\
3=(\text { Fach-)Hochschulstudium } \\
4=\text { Promotion }\end{array}$ \\
\hline
\end{tabular}

Anmerkung: t0: vor der Fortbildung; t1: direkt nach der Fortbildung; t2: 2-5 Monate nach der Fortbildung; MZP: Messzeitpunkt. 


\section{Merkmale auf organisationaler Ebene}

Aus der Vielzahl von möglichen relevanten organisationalen Kontextfaktoren mit Bezug zur Kompetenzentwicklung wurden vier Aspekte in die vorliegende Untersuchung aufgenommen:

- die berufliche Funktion in der Einrichtung (pädagogische Fachkraft, Lehrkraft oder Leitungskraft),

- die Verankerung von MINT-Bildungsinhalten in der Einrichtungskonzeption,

- der allgemeine Betreuungsschlüssel und

- der MINT-bezogene Betreuungsschlüssel.

Aufgrund der mittleren bis hohen Korrelation zwischen der beruflichen Funktion und dem Einrichtungstyp (pädagogische Fachkraft und Kita: $r=.39, p<.01$; Lehrkraft und Grundschule: $r=.68, p<.01$ ) wurde nur die berufliche Funktion als Variable in die Analysen aufgenommen. Die meisten pädagogischen Fachkräfte sind im Krippen- beziehungsweise Kita-Bereich tätig, die befragten Lehrkräfte arbeiten fast ausschließlich an der Grundschule (siehe Abschnitt B 2.3). Die berufliche Funktion bildet also zu einem großen Teil gleichzeitig auch den Einrichtungstyp $a b$, in dem die Befragten tätig sind (Details siehe Abschnitt B 2.3).

In Tabelle 27 wird die Operationalisierung der organisationalen Merkmale näher dargestellt.

Zum einen konnte durch die genannte Auswahl der Kontextfaktoren näher untersucht werden, welchen Zusammenhang die Verankerung der MINT-Bildung in der Einrichtungskonzeption mit der Kompetenzentwicklung des pädagogischen Personals hat. Andererseits wurden mithilfe der beiden Betreuungsschlüssel die personellen Kapazitäten für die Umsetzung von MINT-Bildung im Alltag in der Analyse berücksichtigt. Der allgemeine Betreuungsschlüssel gibt das Verhältnis zwischen der Anzahl aller Pädagoginnen und Pädagogen und der Anzahl der betreuten Kinder in der Einrichtung wieder. In Ergänzung dazu ist der MINT-bezogene Betreuungsschlüssel das Verhältnis zwischen der Anzahl derjenigen Pädagoginnen und Pädagogen, die zu MINT-Bildungsinhalten entdecken und forschen, und der Anzahl der betreuten Kinder in der Einrichtung. 
Tabelle 27. Erhebung der verwendeten unabhängigen Variablen auforganisationaler Ebene

\begin{tabular}{|c|c|c|}
\hline Merkmal & MZP & Inhalt/Kodierung \\
\hline $\begin{array}{l}\text { Aktuelle berufliche } \\
\text { Funktion }\end{array}$ & to, t2 & $\begin{array}{l}\text { Funktion, in der die befragte Person aktuell in der Einrichtung } \\
\text { tätig ist (Mehrfachnennung möglich) } \\
\text { — pädagogische Fachkraft beziehungsweise Erzieher/in } \\
\text { — Lehrer/in (oder Referendar/in) } \\
\text { — Auszubildende/r oder Praktikant/in } \\
\text { n Einrichtungsleitung oder stellvertretende Leitung } \\
\text { n andere Funktion (mit offener Antwortmöglichkeit) }\end{array}$ \\
\hline $\begin{array}{l}\text { MINT-Bildungs- } \\
\text { inhalte in Konzeption }\end{array}$ & to & $\begin{array}{l}\text { schriftliche Einbindung von mathematischen, informati- } \\
\text { schen, naturwissenschaftlichen oder technischen Bildungs- } \\
\text { inhalten in der pädagogischen Konzeption der Einrichtung } \\
\text { (ja/ nein beziehungsweise weiß nicht) }\end{array}$ \\
\hline Betreuungsschlüssel & t2 & $\begin{array}{l}\text { Verhältnis aus } \\
\text { Anzahl der pädagogischen Fach- beziehungsweise Lehr- } \\
\text { kräfte in der Einrichtung (offene Antwort) } \\
\text { — Anzahl der Kinder in der Einrichtung (vier Altersstufen, } \\
\text { jeweils offene Antwort) }\end{array}$ \\
\hline $\begin{array}{l}\text { MINT-bezogener } \\
\text { Betreuungsschlüssel }\end{array}$ & $\mathrm{t} 2$ & $\begin{array}{l}\text { Verhältnis aus } \\
\text { Anzahl der pädagogischen Fach- beziehungsweise Lehr- } \\
\text { kräfte in der Einrichtung, die regelmäßig zu Inhalten aus dem } \\
\text { Bereich Mathematik, Informatik, Naturwissenschaften oder } \\
\text { Technik entdecken und forschen (offene Antwort) } \\
\text { Anzahl der Kinder in der Einrichtung (vier Altersstufen, } \\
\text { jeweils offene Antwort) }\end{array}$ \\
\hline
\end{tabular}

Anmerkung: t2:

2-5 Monate nach der Fortbildung; MZP: Messzeitpunkt.

Die Interpretation beider Betreuungsschlüssel erfolgte unter der Annahme, dass ein höherer Betreuungsschlüssel (weniger Kinder pro pädagogischer Fach- oder Lehrkraft) zu potenziell mehr Zeit für Interaktionen pro Kind führt. Gemäß des Wirkungsmodells besteht die Annahme, dass mehr potenzielle Zeit für mehr MINTbezogene Interaktionen eine wichtige Rahmenbedingung der Kompetenzentwicklung darstellen (vgl. Fröhlich-Gildhoff et al., 2014). Zusätzlich ist anzunehmen, dass dies für alle betrachteten Einrichtungstypen in gleichem Maße gilt. Der allgemeine sowie der MINT-bezogene Betreuungsschlüssel sollten dabei nicht als absolute Werte interpretiert werden, da Aspekte wie Arbeitszeit der Pädagoginnen und Pädagogen oder Betreuungsumfang der Einrichtung nicht berücksichtigt werden. Vielmehr sind die beiden Schlüssel in den folgenden Regressionsanalysen im direkten Vergleich zueinander zu interpretieren: Der allgemeine Betreuungsschlüssel repräsentiert die potenzielle Zeit, die den Pädagoginnen und Pädagogen neben anderen Tätigkeiten für Interaktionen aller Art mit Kindern zur Verfügung steht. Im Rahmen der Regressionsanalysen lieferte der MINT-bezogene Betreuungsschlüssel unter statistischer Kontrolle des allgemeinen Betreuungs- 
schlüssels einen Hinweis darauf, wie viel Zeit den Pädagoginnen und Pädagogen potenziell für MINT-bezogene Aktivitäten pro Kind - im Vergleich zu Interaktionen aller Art - zur Verfügung steht. Es lässt sich annehmen, dass ein höherer Anteil an pädagogischen Fach- oder Lehrkräften, die zu MINT-Themen entdecken und forschen, damit einhergeht, dass potenziell auch mehr Zeit für MINT-spezifischen Austausch im Team zur Verfügung steht.

Der hier vorliegende Bericht fokussiert auf die erfassten Selbsteinschätzungen zu den genannten Variablen. Um Kompetenzen mit möglichst wenig Verzerrung von Urteilsfehlern zu erfassen (vgl. Bortz \& Döring, 2006), wurden in der Längsschnittbefragung auch situationsspezifische Vignetten zur Erfassung von alltagsnahem Verhalten eingesetzt. Diese werden in weiteren Analysen ausgewertet.

\subsection{Stichprobenbeschreibung}

Ziel der Längsschnittbefragung ist es, dieselben Pädagoginnen und Pädagogen über mehrere Jahre hinweg in regelmäßigen Abständen zu den langfristigen Wirkungen der Fortbildungsbesuche zu befragen.

Im Folgenden wird die Stichprobe der pädagogischen Fach-, Lehr- und Leitungskräfte beschrieben, die an allen drei Messzeitpunkten der Längsschnittbefragung teilgenommen haben $(\mathrm{N}=453)$. Relevante Merkmale zur Beschreibung dieser Stichprobe in Hinblick auf die folgenden Analysen werden in Tabelle 28 bis Tabelle 31 aufgeführt. Zur Einschätzung der Repräsentativität der Stichprobe wird die Verteilung der Merkmale zum einen mit dem pädagogischen und leitenden Personal in Kitas und Horten deutschlandweit (vgl. Autorengruppe Fachkräftebarometer, 2019) sowie zum anderen mit stiftungseigenen Monitoring-Daten zu den teilnehmenden Pädagoginnen und Pädagogen am Fortbildungsprogramm der Bildungsinitiative verglichen.

\section{Geschlecht}

Der überwiegende Teil der Befragten (93 Prozent) ist weiblich (siehe Tabelle 28). Pädagogische Fach-, Lehr- und Leitungskräfte unterscheiden sich in dieser Stichprobe nicht hinsichtlich ihres Geschlechts. Die Stichprobe ist mit dem pädagogischen und leitenden Personal in Kitas und Horten in ganz Deutschland bezüglich der Geschlechterverteilung vergleichbar (2018: 6.2 Prozent männlich, 93.8 Prozent weiblich; vgl. Autorengruppe Fachkräftebarometer, 2019). Bei einer stiftungseigenen Befragung von knapp 3.000 Pädagoginnen und Pädagogen 2015 waren 96 Prozent der Befragten weiblich (vgl. Stiftung Haus der kleinen Forscher, 2015). In diesen stiftungseigenen Befragungen spiegelt sich die bundesweite Entwicklung zu einem größeren Anteil von männlichem Personal in Kindertages- 
einrichtungen wider: Dieser war 2015 auch bundesweit kleiner als 2018 (2015: 5.2 Prozent, vgl. Autorengruppe Fachkräftebarometer, 2019).

Tabelle 28. Stichprobenbeschreibung auf individueller Ebene (Teil 1)

\begin{tabular}{lcc}
\hline & N & Prozent \\
\hline Geschlecht & 373 & \\
Weiblich & & $93 \%$ \\
Männlich & & $7 \%$ \\
\hline Höchster Bildungsabschluss & 422 & \\
Ohne Ausbildung & & $2 \%$ \\
Ausbildung & & $65 \%$ \\
Studium & $34 \%$ \\
Promotion & $0 \%$ \\
\hline
\end{tabular}

\section{Bildungsabschluss}

Ein Großteil der Befragten (65 Prozent) verfügt als höchsten Bildungsabschluss über eine Ausbildung. Dennoch ist der Anteil der Befragten mit Studienabschluss (34 Prozent) in dieser Stichprobe recht hoch. Die Befragten aus den unterschiedlichen Berufsgruppen (pädagogische Fach-, Lehr- oder Leitungskräfte) unterscheiden sich dabei signifikant voneinander ( $p<.05)$ : Während von den pädagogischen Fachkräften 22 Prozent einen Hochschulabschluss haben, sind es bei den Einrichtungsleitungen 36 Prozent. Bei einer Stiftungsbefragung von pädagogischen Fach- und Lehrkräften 2015 verfügten ebenfalls 22 Prozent über einen Hochschulabschluss (vgl. Stiftung Haus der kleinen Forscher, 2015). Diese Stichproben aus stiftungseigenen Befragungen weisen einen deutlich höheren Anteil an pädagogischen Fachkräften und Leitungskräften mit Hochschulabschluss als im Bundesdurchschnitt auf: Bundesweit verfügten 2018 knapp sechs Prozent des pädagogischen und leitenden Personals in Kindertageseinrichtungen in Deutschland über einen einschlägigen Hochschulabschluss (vgl. Autorengruppe Fachkräftebarometer, 2019). Von den Lehrkräften haben fast alle Befragten (98 Prozent) ein Studium absolviert.

\section{Alter}

Die befragten Pädagoginnen und Pädagogen sind durchschnittlich etwa 43 Jahre alt und seit knapp 13 Jahren in ihrer aktuellen beruflichen Funktion tätig (siehe Tabelle 29). Pädagogische Fach-, Lehr- und Leitungskräfte unterscheiden sich in dieser Stichprobe hinsichtlich ihres Alters. So sind die pädagogischen Fachkräfte mit gut 41 Jahren durchschnittlich signifikant jünger als die Leitungskräfte mit durchschnittlich rund 48 Jahren $(p<.05)$. Die Lehrkräfte sind im Mittel 46 Jahre alt und unterscheiden sich nicht von den beiden anderen Berufsgruppen. Damit deckt sich das Alter der teilnehmenden pädagogischen Fachkräfte in etwa mit für 
2016 berichteten Daten für Deutschland, wonach das Durchschnittsalter der Fachkräfte aus dem Bereich frühe Bildung 41 Jahre betrug (vgl. Autorengruppe Fachkräftebarometer, 2019), sowie mit früheren Stiftungserhebungen (Durchschnittsalter: 45 Jahre, vgl. Stiftung Haus der kleinen Forscher, 2015). Hinsichtlich ihrer Berufserfahrung unterscheiden sich die Befragten mit unterschiedlicher beruflicher Funktion hingegen nicht.

Tabelle 29. Stichprobenbeschreibung auf individueller Ebene (Teil 2)

\begin{tabular}{lccccc}
\hline & $\mathrm{N}$ & Min & Max & M & SD \\
\hline Alter in Jahren & 369 & 20 & 64 & 42.61 & 11.36 \\
\hline Berufserfahrung in Jahren & 381 & 1 & 45 & 12.79 & 10.92 \\
\hline Fortbildungsanzahl vor t0 & 445 & 0 & 17 & 2.84 & 3.72 \\
\hline $\begin{array}{l}\text { Fortbildungsanzahl zwi- } \\
\text { schen t1 und t2 }\end{array}$ & 420 & 0 & 3 & 0.18 & 0.47 \\
\hline Fortbildungsfrequenz vor t0 & 434 & 0 & 6 & 0.78 & 0.89 \\
\hline
\end{tabular}

Anmerkung: t0: vor der Fortbildung; t1: direkt nach der Fortbildung; t2: 2-5 Monate nach der Fortbildung

\section{Anzahl besuchter Fortbildungen}

Zum Zeitpunkt der Ersterhebung (to/t1) haben die Befragten durchschnittlich knapp drei Präsenzfortbildungen der Initiative „Haus der kleinen Forscher“ besucht. Hierbei ist die Spannweite recht groß: Acht Prozent der Befragten haben an zehn oder mehr Präsenzfortbildungen teilgenommen, während mit 40 Prozent der Großteil der Stichprobe Fortbildungsneulinge sind, die ihre erste Fortbildung besuchen.

Die Anzahl besuchter Fortbildungen steigt bis zum Zeitpunkt der Folgebefragung (t2) zwei bis fünf Monate später auf knapp vier Fortbildungen. Dies ist zum einen darin begründet, dass hierbei die zum Zeitpunkt der Ersterhebung (t0/t1) besuchte Fortbildung, in deren Rahmen die Befragung stattfand, berücksichtigt wird. Darüber hinaus haben etwa 15 Prozent der Befragten zwischen der Ersterhebung ( $\mathrm{t} 0 / \mathrm{t} 1$ ) und der Folgebefragung ( $\mathrm{t} 2$ ) zwei bis fünf Monate später zwischen einer und drei weiteren „Haus der kleinen Forscher“-Fortbildungen besucht.

Da viele Pädagoginnen und Pädagogen im Laufe der Zeit weitere Fortbildungen besuchen, wird der Gesamtanteil der Fortbildungsneulinge im Sample perspektivisch kleiner werden und nimmt vor allem zu den ersten Messzeitpunkten dieser langfristig angelegten Befragung einen größeren Anteil ein. Der vergleichsweise hohe Anteil an Pädagoginnen und Pädagogen, die zuvor noch keine „Haus der kleinen Forscher"-Fortbildung besucht haben, wurde für den ersten Messzeitpunkt der Längsschnittbefragung bewusst forciert, indem für die Ersterhebung lokale Netzwerke ausgewählt wurden, die im Vorjahr eine hohe Quote an Fortbil- 
dungsneulingen aufwiesen. Wie stiftungseigene Monitoring-Daten zeigen, steigt die Anzahl der besuchten Fortbildungen mit der Teilnahmedauer am Bildungsprogramm der Initiative kontinuierlich (Stiftung Haus der kleinen Forscher, 2014, 2017).

\section{Fortbildungsfrequenz}

Im Durchschnitt hatten die befragten Pädagoginnen und Pädagogen zum Zeitpunkt der Ersterhebung (t0/t1) knapp eine Fortbildung pro Jahr ihrer bisherigen Teilnahmedauer besucht. Die Spannweite ist dabei mit einer bis sechs besuchten Fortbildungen pro Jahr recht groß. Die Fortbildungsfrequenz (Anzahl besuchter Fortbildungen pro Jahr) der Befragten deckt sich mit Daten aus einer stiftungseigenen Befragung von 2016 von 2.100 Pädagoginnen und Pädagogen aus Kitas, Horten und Grundschulen; hier lag die durchschnittliche Fortbildungsfrequenz bei 0.9 Fortbildungen pro Jahr (vgl. Stiftung Haus der kleinen Forscher, 2017). Pädagogische Fachkräfte sowie Einrichtungsleitungen unterscheiden sich in der vorliegenden Untersuchung hinsichtlich der Fortbildungsfrequenz signifikant von den befragten Lehrkräften ( $p<.05)$ : Während die pädagogischen Fachkräfte zum Zeitpunkt der Ersterhebung (to/t1) durchschnittlich 3.1 und die Einrichtungsleitungen 4.3 Fortbildungen besucht haben, sind es bei den Lehrkräften 1.2 besuchte Fortbildungen. Auch unterscheidet sich die Fortbildungsfrequenz: Die Einrichtungsleitungen haben jährlich 1.1 Fortbildungen besucht, während es bei den Lehrkräften 0.6 Fortbildungen pro Jahr sind. Mit 0.8 besuchten Fortbildungen pro Jahr unterscheiden sich die pädagogischen Fachkräfte nicht von den anderen Berufsgruppen. Hinsichtlich der zwischen der Ersterhebung (to/t1) und der Folgeerhebung (t2) besuchten Fortbildungen bei der Initiative „Haus der kleinen Forscher" unterscheiden sich die Personen aus den verschiedenen Berufsgruppen nicht.

\section{Berufliche Funktion}

Mit etwa zwei Dritteln (67 Prozent) handelt es sich bei den an der Längsschnittbefragung teilnehmenden Pädagoginnen und Pädagogen mehrheitlich um pädagogische Fachkräfte (siehe Tabelle 30). Die Anteile an Lehrkräften beziehungsweise Leitungskräften sind mit weiteren 11 beziehungsweise 14 Prozent ähnlich groß. Fünf Prozent der Befragten haben - teilweise zusätzlich zu den aufgeführten beruflichen Funktionen - eine sonstige Funktion angegeben, beispielsweise Kinderpfleger beziehungsweise -pflegerin, Erzieherhelfer beziehungsweise -helferin, Sprachförderkraft oder Heilpädagoge beziehungsweise -pädagogin. Die meisten Befragten arbeiten im Kita-Bereich (74 Prozent), daneben sind je 15 Prozent im Krippenbereich sowie in der Grundschule tätig (Mehrfachnennung möglich). Im Hort arbeiten neun Prozent. Die meisten Befragten betreuen Kinder im Alter zwi- 
schen drei und sechs Jahren (72 Prozent), etwa ein Drittel der Befragten betreut (teilweise zusätzlich) unter dreijährige Kinder (34 Prozent). Ein weiteres knappes Drittel der Befragten betreut Kinder im Grundschulalter (28 Prozent), nur sieben Prozent betreuen Kinder, die älter als zehn Jahre sind.

Berufliche Funktion der Befragten und Einrichtungstyp überschneiden sich zu großen Teilen: So sind 90 Prozent der pädagogischen Fachkräfte im Krippenbeziehungsweise Kita-Bereich tätig, 96 Prozent der Lehrkräfte im Grundschulbereich. Von den Leitungskräften sind 84 Prozent in Krippe beziehungsweise Kita tätig, 16 Prozent (teilweise zusätzlich) im Hort sowie zwölf Prozent in der Grundschule. Aufgrund dieser großen Überschneidung wird die Variable berufliche Funktion in den folgenden Analysen als Merkmal der Organisation eingeordnet.

Tabelle 30. Stichprobenbeschreibung auforganisationaler Ebene (Teil 1)

\begin{tabular}{|c|c|c|}
\hline & $\mathrm{N}$ & Prozent \\
\hline Berufliche Funktion & 448 & \\
\hline Pädagogische Fachkraft & & $67 \%$ \\
\hline Lehrkraft & & $11 \%$ \\
\hline Leitung & & $14 \%$ \\
\hline Sonstige Funktion & & $5 \%$ \\
\hline Einrichtungstyp ${ }^{a}$ & 448 & \\
\hline Krippe & & $15 \%$ \\
\hline Kindergarten & & $74 \%$ \\
\hline Hort & & $9 \%$ \\
\hline Grundschule & & $15 \%$ \\
\hline Alter der betreuten Kinder ${ }^{\mathrm{a}}$ & 448 & \\
\hline 0 bis unter 3 Jahre & & $34 \%$ \\
\hline 3 bis unter 6 Jahre & & $72 \%$ \\
\hline 6 bis unter 10 Jahre & & $28 \%$ \\
\hline 10 Jahre und älter & & $7 \%$ \\
\hline
\end{tabular}

Anmerkung: aAnteil entspricht der Zustimmung zum Einzelitem (Mehrfachnennung möglich).

\section{MINT-Bildung in der Organisation}

81 Prozent der Befragten gaben an, dass MINT-Bildungsinhalte in der Einrichtungskonzeption verankert sind (siehe Tabelle 31). Personen aus den verschiedenen Berufsgruppen unterscheiden sich dabei nicht signifikant voneinander. Der allgemeine Betreuungsschlüssel sagt aus, dass jedes Kind durchschnittlich von 0.15 Pädagoginnen beziehungsweise Pädagogen betreut wird; dies entspricht einem Verhältnis von einer Pädagogin beziehungsweise einem Pädagogen zu gut sechs Kindern. Betrachtet man die Anzahl der Pädagoginnen und Pädagogen, die in der Einrichtung zu MINT-Bildungsinhalten entdecken und forschen, im Verhältnis zur Anzahl der Kinder, so ergibt sich im Durchschnitt ein MINT-bezogener Betreuungsschlüssel von 0.07 (etwa eine Pädagogin beziehungsweise ein Pädagoge zu 14 Kindern). Diese beiden Werte können jeder für sich genommen nicht sinn- 
voll interpretiert werden. Sie erheben aufgrund ihrer Berechnung, wie beschrieben, nicht den Anspruch, die reale Fachkraft-Kind-Relation in den Einrichtungen abzubilden, sondern geben im direkten Vergleich miteinander Hinweise darauf, wie viel Zeit den Pädagoginnen und Pädagogen für MINT-bezogene Interaktionen im Vergleich zur Zeit für pädagogische Interaktionen aller Art zur Verfügung steht.

Bei den Betreuungsschlüsseln zeigen sich signifikante Unterschiede zwischen den in der Grundschule tätigen Lehrkräften im Vergleich zu den pädagogischen Fachkräften und Einrichtungsleitungen, die überwiegend im Krippen- und Kita-Bereich tätig sind ( $p<.05$ ): Die Lehrkräfte geben an, dass in den Grundschulen mit einem Betreuungsschlüssel mit etwa einer Lehrkraft je elf Kinder deutlich mehr Kinder von einer Pädagogin beziehungsweise einem Pädagogen betreut werden als im Kita-Bereich mit etwa einer pädagogischen Fachkraft pro sechs Kindern. Dieses Verhältnis spiegelt sich auch im MINT-bezogenen Betreuungsschlüssel wider (durchschnittlich 0.04 bei den Lehrkräften, 0.07 bei den pädagogischen Fachkräften). Dies legt die Annahme nahe, dass den Lehrkräften potenziell deutlich weniger Zeit für MINT-spezifische Interaktionen pro Kind zur Verfügung steht. Die Korrelation beider Schlüssel ist mit $r=.45(p<.01)$ von mittlerer Größe.

Tabelle 31. Stichprobenbeschreibung auforganisationaler Ebene (Teil 2)

\begin{tabular}{lccccc}
\hline & N & Min & Max & M & SD \\
\hline MINT-Bildungsinhalte in Konzeption & 429 & .00 & 1.00 & .81 & .40 \\
\hline Betreuungsschlüssel (zentriert) & 364 & .03 & .46 & .15 & .06 \\
\hline $\begin{array}{l}\text { MINT-bezogener Betreuungsschlüssel } \\
\text { (zentriert) }\end{array}$ & 364 & .00 & .44 & .07 & .06 \\
\hline
\end{tabular}

Insgesamt ähneln die Teilnehmenden der Längsschnittbefragung den bundesweit tätigen pädagogischen Fachkräften in Hinblick auf Geschlecht und Alter. Ein Unterschied zeigt sich im höchsten erreichten Bildungsabschluss mit einem höheren Anteil von Personen mit Hochschulabschluss in dieser Stichprobe im Vergleich zum Bundesdurchschnitt. Ein Vergleich der untersuchten Längsschnittstichprobe zu weiteren stiftungseigenen Monitoring-Daten bezüglich der am Bildungsprogramm der Stiftung teilnehmenden Pädagoginnen und Pädagogen zeigt, dass sich diese hinsichtlich der Fortbildungsfrequenz (Anzahl besuchter Fortbildungen pro Jahr) ähneln. Leichte Unterschiede zeigen sich hingegen dahingehend, dass die Stichprobe der Längsschnittbefragung einen etwas höheren Anteil an Männern aufweist. Zudem verfügen mehr Pädagoginnen und Pädagogen über einen Studienabschluss und sind etwas jünger als in einer stiftungseigenen Befragung von 2015.

Die Besonderheiten dieser Stichprobe sind für die Interpretation der Ergebnisse zur ersten Forschungsfrage zu berücksichtigen. Die Analysen zur Beantwor- 
tung der zweiten und dritten Forschungsfrage wurden unter Konstanthaltung dieser Merkmale durchgeführt, weshalb die Besonderheiten dieser Stichprobe bei der Ergebnisinterpretation nicht berücksichtigt werden müssen.

\subsection{Analyseverfahren}

Die Teilnahmeeffekte des Fortbildungsbesuchs auf die Kompetenzentwicklung der befragten Pädagoginnen und Pädagogen wurden mithilfe von Varianz- und Regressionsanalysen untersucht. In der Analyse wurden ausschließlich Daten von Personen berücksichtigt, welche zu allen drei Messzeitpunkten (t0, t1, t2) an der Befragung teilgenommen haben.

Um Fragestellung 1 (siehe Abschnitt B 1.2) zu beantworten, wurden in einem ersten Schritt mithilfe einer Varianzanalyse die durchschnittlichen kurz- und mittelfristigen Fortbildungseffekte für die Gesamtstichprobe berechnet, welche den Zuwachs in verschiedenen Kompetenzen vor und nach der Teilnahme an einer Fortbildung widerspiegeln (siehe Ergebnisse in Abschnitt B 3.1). Als varianzanalytisches Verfahren kamen einfaktorielle Varianzanalysen mit Messwiederholung zur Anwendung (Repeated Measures ANOVA).

Da sich die berechneten Fortbildungseffekte für Personen mit Bezug zu deren individuellen Eigenschaften und organisationalen Rahmenbedingungen unterscheiden können (Fragestellung 2), wurden unter Anwendung von regressionsanalytischen Verfahren (multiple Regressionsmodelle) Zusammenhänge zwischen diesen Merkmalen und den Fortbildungseffekten berechnet (siehe Ergebnisse in Abschnitt B 3.2). Mithilfe dieses Verfahrens können Effekte des Fortbildungsbesuchs unter Berücksichtigung von Kontrollvariablen identifiziert werden, wodurch eine validere Aussage über die Wirkungen der Teilnahme an den Fortbildungen ermöglicht wird.

Für die Beantwortung der Fragestellungen 1 und 2 wurden folgende Fortbildungseffekte näher untersucht:

- kurzfristiger Effekt (t0 zu t1): die Differenz des Kompetenzniveaus zwischen vor und direkt nach der Teilnahme an der Fortbildung,

- mittelfristiger Effekt (t0 zu t2): die Differenz des Kompetenzniveaus zwischen vor und zwei bis fünf Monate nach der Teilnahme an der Fortbildung.

In Hinblick auf Fragestellung 3 wurde näher untersucht, inwieweit ein Zusammenhang zwischen der Fortbildungsanzahl und -frequenz vor dem betrachteten Fortbildungsbesuch mit dem selbsteingeschätzten Ausgangsniveau der Kompetenzen der befragten Pädagoginnen und Pädagogen zum Messzeitpunkt to besteht (siehe Ergebnisse in Abschnitt B 3.3). Die Ergebnisse liefern erste Hinweise auf 
mögliche längerfristige Effekte der Fortbildungsteilnahme. Zudem wurde untersucht, ob individuelle Eigenschaften oder organisationale Rahmenbedingungen in Zusammenhang mit dem Ausgangsniveau (t0) der Befragten stehen. Hierbei kamen ebenso regressionsanalytische Verfahren zur Anwendung.

Im Folgenden werden zwei Signifikanzniveaus berichtet: Ergebnisse mit $p<$ $\left..05{ }^{\star \star}\right)$ und $p<.10\left(^{*}\right)$ werden als signifikant berichtet. Die beiden Signifikanzniveaus sind in den Ergebnisdarstellungen durch entsprechende Symbole gekennzeichnet. Neben der Signifikanz bemisst sich die Bedeutsamkeit eines Effekts an der Effektstärke. Für die Bestimmung der Effektstärke von Mittelwertunterschieden und zur Beurteilung ihrer praktischen Relevanz wurde Cohens $d$ herangezogen. Nach Cohen (1988) spricht man ab einem Wert von 0.2 von einem kleinen, ab 0.5 von einem mittleren und ab 0.8 von einem starken Effekt. Zur Einordnung der praktischen Relevanz der Ergebnisse der Regressionsanalysen in den Abschnitten B 3.2 und B 3.3 wird das Effektstärkemaß Cohens $f^{2}$ herangezogen, das die Größe des statistischen Effekts beschreibt. Ab einem $f^{2}$ von 0.02 spricht man von einem kleinen, $a b 0.15$ von einem mittleren und ab 0.35 von einem starken Effekt (vgl. Chin, 1998). 


\section{Ergebnisse}

Im Folgenden werden in einem ersten Schritt die Ergebnisse der varianzanalytischen Verfahren bezüglich der durchschnittlichen kurz- und mittelfristigen Effekte der Teilnahme an einer Präsenzfortbildung der Gesamtstichprobe berichtet (siehe Abschnitt B 3.1). Die berechneten Effektstärken stellen unbereinigte Effekte des untersuchten Fortbildungsbesuchs dar und geben erste Hinweise auf die Wirkungen der Fortbildung. In einem zweiten Schritt werden die Zusammenhänge von bestimmten individuellen Eigenschaften und organisationalen Rahmenbedingungen mit den kurz- und mittelfristigen Fortbildungseffekten berichtet, welche mithilfe von regressionsanalytischen Verfahren ermittelt wurden (siehe Abschnitt B 3.2). Abschließend wird anhand des Ausgangsniveaus der Kompetenzen beispielhaft untersucht, inwieweit ein Zusammenhang zwischen der Fortbildungsanzahl beziehungsweise -frequenz vor dem betrachteten Fortbildungsbesuch (t0) und dem selbsteingeschätzten Kompetenzniveau besteht, um erste Erkenntnisse in Hinblick auf längerfristige Fortbildungseffekte zu gewinnen (siehe Abschnitt B 3.3).

\subsection{Kurz- und mittelfristige Kompetenzentwicklung nach einem Fortbildungsbesuch}

Um Fragestellung 1 zu beantworten, werden in diesem Abschnitt die durchschnittlichen Fortbildungseffekte der befragten Pädagoginnen und Pädagogen für das fachdidaktische Wissen, die Motivation und die Selbstwirksamkeit in Bezug auf das Entdecken und Forschen mit Kindern berichtet. Abbildung 20 gibt auf einer Skala von 1 (trifft überhaupt nicht zu) bis 6 (trifft voll und ganz zu) die durchschnittliche Entwicklung der verschiedenen Kompetenzen direkt nach (t1) und einige Monate nach (t2) einem einzelnen Fortbildungsbesuch wieder. 


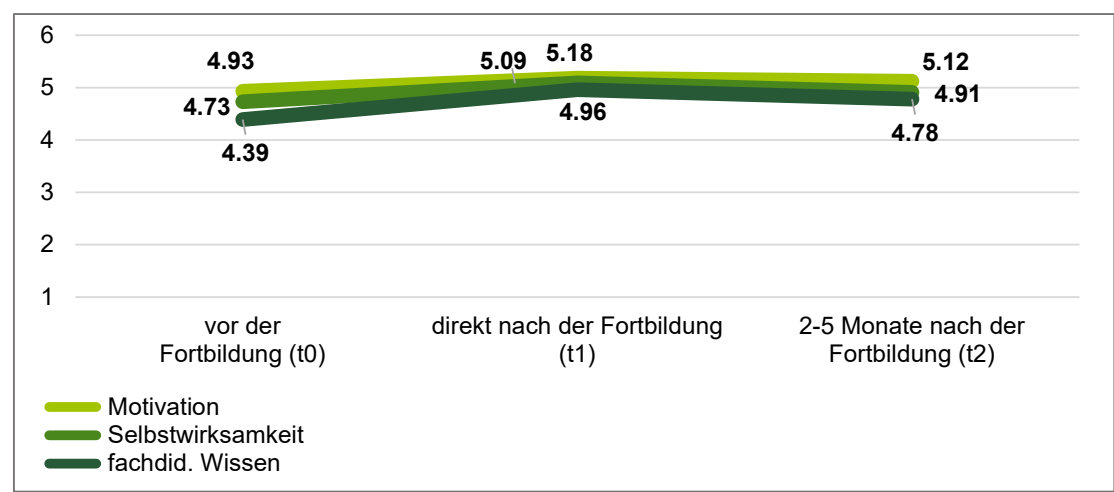

Abbildung 20. Entwicklung verschiedener Kompetenzen nach einem Fortbildungsbesuch ( $N$ = 379-383); fachdidaktisches Wissen, Motivation und Selbstwirksamkeit beziehen sich auf das Entdecken und Forschen mit Kindern

\section{Fachdidaktisches Wissen zum Entdecken und Forschen}

Die Befragten schätzen ihr fachdidaktisches Wissen in Bezug auf das Entdecken und Forschen mit Kindern bereits vor dem Besuch einer Fortbildung relativ hoch ein $(M=4.39)$. Der durchschnittliche kurzfristige Teilnahmeeffekt, also der Zuwachs von vor der Fortbildung zu direkt nach der Fortbildung (t0 zu t1), ist für das fachdidaktische Wissen signifikant (steigt um 0.57 auf $M=4.96$ ). Zwischen 11 und t2 zeigt sich ein kleiner, aber signifikanter Abfall des Mittelwerts (um 0.18 auf $M=$ 4.78); die befragten Pädagoginnen und Pädagogen schätzen ihr fachdidaktisches Wissen in Bezug auf das Entdecken und Forschen mit Kindern einige Monate später also etwas geringer ein als unmittelbar nach der Fortbildung. Insgesamt ist zwischen dem Ausgangswert vor dem Fortbildungsbesuch und dem Wert einige Monate später (t0 zu t2) mittelfristig ein stabiler signifikanter Zuwachs (um 0.39) zu erkennen. Für das selbsteingeschätzte fachdidaktische Wissen kann also sowohl kurz- als auch mittelfristig ein bedeutsamer Fortbildungseffekt festgestellt werden. Insgesamt zeigen sich für die Entwicklung des fachdidaktischen Wissens nach einem Fortbildungsbesuch durchschnittlich mittelgroße Effekte (kurzfristig: Cohens $d=0.73$, mittelfristig: Cohens $d=0.52$, vgl. Abschnitt B 2.4).

Tabelle 32 gibt einen Gesamtüberblick zum Ausgangsniveau der Kompetenzen vor der Fortbildung (t0) sowie deren Entwicklung nach dem Fortbildungsbesuch zu $\mathrm{t} 1$ und $\mathrm{t} 2$. 
Tabelle32. Ausgangsniveauunddurchschnittliche TeilnahmeeffektedesFortbildungsbesuchs für die Kompetenzen fachdidaktisches Wissen, Motivation und Selbstwirksamkeit

\begin{tabular}{|c|c|c|c|c|}
\hline & Min & Max & M & SD \\
\hline \multicolumn{5}{|c|}{ Fachdidaktisches Wissen $\left(N=380, F=114.26^{\star \star}\right)$} \\
\hline Ausgangsniveau (t0) & 1.5 & 6.0 & 4.39 & 0.84 \\
\hline Kurzfristiger Effekt (t0 zu t1) & -1.0 & 3.0 & $0.57 * *$ & 0.66 \\
\hline Mittelfristiger Effekt (t0 zu t2) & -1.5 & 3.0 & $0.39 * *$ & 0.70 \\
\hline \multicolumn{5}{|l|}{ Motivation $(N=383, F=28.83 * *)$} \\
\hline Ausgangsniveau (t0) & 2.0 & 6.0 & 4.93 & 0.73 \\
\hline Kurzfristiger Effekt (t0 zu t1) & -1.5 & 2.0 & $0.25 * *$ & 0.66 \\
\hline Mittelfristiger Effekt (to zu t2) & -2.0 & 3.0 & 0.20 ** & 0.69 \\
\hline \multicolumn{5}{|c|}{ Selbstwirksamkeit $\left(N=379, F=44.71^{\star \star}\right)$} \\
\hline Ausgangsniveau (t0) & 2.5 & 6.0 & 4.73 & 0.78 \\
\hline Kurzfristiger Effekt (t0 zu t1) & -1.5 & 2.5 & $0.36 * *$ & 0.62 \\
\hline Mittelfristiger Effekt (to zu t2) & -2.5 & 3.0 & $0.18 * \star$ & 0.68 \\
\hline
\end{tabular}

Anmerkung: ${ }^{* \star} p<.05$; t0: vor der Fortbildung; t1: direkt nach der Fortbildung; t2: 2-5 Monate nach der Fortbildung; fachdidaktisches Wissen, Motivation und Selbstwirksamkeit beziehen sich auf das Entdecken und Forschen mit Kindern.

\section{Motivation zum Entdecken und Forschen}

Die befragten Pädagoginnen und Pädagogen geben bereits vor der Teilnahme an der Fortbildung an, hoch motiviert zu sein, mit Kindern zu entdecken und zu forschen $(M=4.93)$. Der Mittelwert steigt nach dem Besuch einer Fortbildung signifikant an (um 0.25 auf $M=5.18$ ) und bleibt einige Monate nach dem Fortbildungsbesuch stabil bestehen (das leichte Absinken des Werts um 0.06 auf $M=5.12$ ist nicht signifikant). Insgesamt lässt sich für den betrachteten Zeitraum von vor der Fortbildung bis einige Monate nach dem Fortbildungsbesuch im Durchschnitt ein signifikanter Anstieg der Motivation feststellen (um 0.19). Insgesamt zeigen sich für die Entwicklung der Motivation durchschnittlich kleine Effekte (kurzfristig: Cohens $d=0.39$, mittelfristig: Cohens $d=0.26$ ).

\section{Selbstwirksamkeit zum Entdecken und Forschen}

Für die Gesamtstichprobe der Pädagoginnen und Pädagogen zeigt sich schon vor der Fortbildung ein hoher Wert für die selbsteingeschätzte Selbstwirksamkeit $(M=4.73)$, d. h., sie trauen sich bereits zu, mit Kindern zu entdecken und zu forschen. Nach dem Fortbildungsbesuch steigt die Selbstwirksamkeit der Pädagoginnen und Pädagogen im Mittel signifikant an (um 0.36 auf $M=5.09$ ). Einige Monate nach dem Fortbildungsbesuch schätzen sie ihre Selbstwirksamkeit wieder leicht geringer ein (um 0.17 auf $M=4.91$, dieser Abfall im Mittelwert ist signifikant). Insgesamt bleibt jedoch für die Gesamtstichprobe mittelfristig (t0 zu t2) 
ein signifikanter Zuwachs der Selbstwirksamkeit (um 0.18) bestehen. Die Effekte für die Entwicklung der Selbstwirksamkeit sind nach Cohen als klein einzuordnen (kurzfristig: Cohens $d=0.49$, mittelfristig: Cohens $d=0.24$ ).

\section{Zusammenfassung und Diskussion}

Bei den befragten Pädagoginnen und Pädagogen zeigen sich sowohl kurz- als auch mittelfristige Fortbildungseffekte auf die Kompetenzentwicklung durch den Besuch einer „Haus der kleinen Forscher“-Fortbildung. Mithilfe von Varianzanalysen kann festgestellt werden, dass sich die Entwicklung der drei untersuchten Kompetenzen fachdidaktisches Wissen, Motivation und Selbstwirksamkeit sowohl direkt nach der Fortbildung (t1) als auch einige Monate später (t2) signifikant vom Ausgangsniveau der Kompetenzen vor der Fortbildung (t0) unterscheidet. Das heißt, die Pädagoginnen und Pädagogen schätzen ihr fachdidaktisches Wissen nach dem Besuch einer „Haus der kleinen Forscher“-Fortbildung im Durchschnitt bedeutsam höher ein, sie empfinden sich kurz- wie mittelfristig als leicht motivierter, mit Kindern zu entdecken und zu forschen, und trauen sich dies auch eher zu.

Die Ergebnisse weisen darauf hin, dass der Besuch einer Fortbildung durchschnittlich einen stärkeren Zusammenhang mit dem fachdidaktischen Wissen hat als mit den Kompetenzen Motivation und Selbstwirksamkeit. Bei der Interpretation der Ergebnisse sollte jedoch beachtet werden, dass die befragten Pädagoginnen und Pädagogen ihre Motivation und Selbstwirksamkeit bereits vor der Fortbildung sehr hoch einschätzten und somit eventuell weniger Möglichkeiten bestanden, sich in Hinblick auf diese Kompetenzen noch stark zu entwickeln.

Inwieweit sich die in Abschnitt B 2.3 berichteten Eigenschaften der Stichprobe, beispielsweise der hohe Anteil von Pädagoginnen und Pädagogen mit Studienabschluss, auf die dargestellte Kompetenzentwicklung auswirken, kann nicht eindeutig geklärt werden.

\subsection{Zusammenhänge individueller Eigenschaften und organisationaler Rahmenbedingungen mit den Wirkungen der Fortbildungsteilnahme}

Die Ergebnisse der in Abschnitt 3.1 berichteten Varianzanalyse geben Hinweise darauf, dass die Teilnahme an einer Fortbildung im Durchschnitt kleine bis mittlere kurz- und mittelfristige Effekte auf das selbsteingeschätzte fachdidaktische Wissen der befragten Pädagoginnen und Pädagogen sowie auf ihre selbsteingeschätzte Motivation und Selbstwirksamkeit in Bezug auf das Entdecken und Forschen mit Kindern ausübt. Diese durchschnittlichen Effekte geben jedoch noch 
keinen Aufschluss darüber, ob es personenspezifische Unterschiede im Lernerfolg gibt und welche individuellen Eigenschaften und organisationalen Rahmenbedingungen mit den Fortbildungswirkungen zusammenhängen.

Mittels regressionsanalytischer Verfahren wird daher in der Folge näher untersucht, welche individuellen Eigenschaften und organisationalen Rahmenbedingungen in einem fördernden oder hemmenden Zusammenhang mit der kurz- und mittelfristigen Kompetenzentwicklung nach einem Fortbildungsbesuch stehen. Hierzu werden die in Abschnitt 2.2 beschriebenen Faktoren auf individueller und organisationaler Ebene in die Analysen mit einbezogen.

Aufgrund des Längsschnittdesigns muss ebenso berücksichtigt werden, dass sich Einflussfaktoren zwischen den Messzeitpunkten ändern können. So können die Pädagoginnen und Pädagogen z. B. zwischen der hier betrachteten Fortbildung und dem Messzeitpunkt einige Monate später ( $\mathrm{t} 2$ ) noch weitere Fortbildungen besucht haben. Als weiterer Faktor wird daher die Anzahl der zwischen $\mathrm{t} 1$ und t2 besuchten Präsenzfortbildungen in den Blick genommen.

Im Folgenden werden für jede der drei Kompetenzen (fachdidaktisches Wissen, Motivation und Selbstwirksamkeit in Bezug auf das Entdecken und Forschen mit Kindern) die Zusammenhänge der individuellen Eigenschaften und organisationalen Rahmenbedingungen mit den kurzfristigen und mittelfristigen Fortbildungseffekten regressionsanalytisch berechnet. Diese Analyse ermöglicht es, sich den Zusammenhang eines Merkmals mit den Fortbildungseffekten bei gleichzeitiger Konstanthaltung der anderen Merkmale anzuschauen.

\section{Fachdidaktisches Wissen in Bezug auf das Entdecken und Forschen}

Zunächst werden die Ergebnisse der Regressionsanalysen zum Zusammenhang zwischen individuellen Eigenschaften sowie organisationalen Rahmenbedingungen und den Wirkungen der Fortbildungsteilnahme auf das fachdidaktische Wissen betrachtet (siehe Tabelle 33).

In Hinblick auf die individuellen Eigenschaften zeigt sich ein Zusammenhang zwischen mittelfristigem Fortbildungseffekt und Fortbildungsanzahl (Anzahl aller vor to besuchten Fortbildungen bei der Initiative „Haus der kleinen Forscher“). Personen mit höherer Anzahl besuchter Fortbildungen vor t0 weisen einen signifikant höheren mittelfristigen Fortbildungseffekt auf (t0 zu t2). Betrachtet man die Kompetenzentwicklung des fachdidaktischen Wissens in einem Zeitraum von zwei bis fünf Monaten nach einem Fortbildungsbesuch, profitieren also Personen von der Teilnahme umso mehr, je mehr Fortbildungen sie vorab bereits besucht haben. 
Tabelle 33. Ergebnisse der Regressionsanalysen zum Zusammenhang zwischen den Wirkungen der Fortbildungsteilnahme auf das fachdidaktische Wissen und individuellen Eigenschaften sowie organisationalen Rahmenbedingungen $(N=294-312)$

\begin{tabular}{|c|c|c|}
\hline & $\begin{array}{c}\text { Kurzfristiger } \\
\text { Effekt (t0 zu t1) }\end{array}$ & $\begin{array}{l}\text { Mittelfristiger } \\
\text { Effekt (to zu t2) }\end{array}$ \\
\hline Konstante & $0.61 * \star$ & $0.29 \star \star$ \\
\hline Fortbildungsanzahl vor t0 & 0.02 & $0.03^{*}$ \\
\hline Fortbildungsanzahl zwischen $\mathrm{t} 1$ und $\mathrm{t} 2$ & 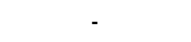 & 0.09 \\
\hline Fortbildungsfrequenz zu to & $-0.10^{* *}$ & -0.01 \\
\hline Wissensstand zu to (z) & $-0.55^{* *}$ & $-0.66 * *$ \\
\hline Berufserfahrung (z) & $-0.01^{*}$ & 0.00 \\
\hline Bildungsabschluss (Studium) & -0.01 & 0.10 \\
\hline Funktion (Lehrkraft) & -0.05 & -0.18 \\
\hline Funktion (Leitung) & 0.07 & -0.10 \\
\hline MINT-Bildungsinhalte in Konzeption & - & 0.03 \\
\hline Betreuungsschlüssel (z) & - & -0.37 \\
\hline MINT-bezogener Betreuungsschlüssel (z) & - & $1.76^{\star \star}$ \\
\hline Korrigiertes $\mathrm{R}^{2}$ & 0.43 & 0.43 \\
\hline
\end{tabular}

Anmerkung: Dargestellt sind unstandardisierte Regressionskoeffizienten $(B) ;{ }^{* \star} p<.05$; ${ }^{*} p<.10$; t0: vor der Fortbildung; t1: direkt nach der Fortbildung; t2: 2-5 Monate nach der Fortbildung; z: Variable zentriert.

Für die Fortbildungsfrequenz (Anzahl der besuchten „Haus der kleinen Forscher“Fortbildungen pro Jahr vor t0) ergeben die Analysen ein gegenteiliges Bild. Personen mit höherer Fortbildungsfrequenz zeigen kurzfristig einen signifikant geringeren Fortbildungseffekt (t0 zu t1). Der kurzfristige Wissenszuwachs fällt also geringer aus, je mehr Fortbildungen sie zuvor pro Jahr besucht haben. Dieser Zusammenhang zeigt sich mittelfristig allerdings nicht mehr, d. h., für den mittelfristigen Zuwachs im fachdidaktischen Wissen nach einem Fortbildungsbesuch scheint die Fortbildungsfrequenz zuvor besuchter Fortbildungen keine Rolle zu spielen.

Das Ausgangsniveau des selbsteingeschätzten fachdidaktischen Wissens zum Messzeitpunkt to scheint in einem wichtigen Zusammenhang mit der weiteren Entwicklung zu stehen. Personen mit einem überdurchschnittlichen selbsteingeschätzten Wissensstand zu to weisen einen signifikant geringeren Fortbildungseffekt für die betrachteten Zeiträume auf. Das heißt, je höher Personen ihr fachdidaktisches Wissen vor einer Fortbildung einschätzen, umso geringer fällt ihr Wissenszuwachs nach einem einzelnen Fortbildungsbesuch aus. Im Umkehrschluss deutet dies darauf hin, dass Personen mit einem geringeren Ausgangsniveau beim fachdidaktischen Wissen mehr von dem Fortbildungsbesuch profitie- 
ren als Personen mit einem bereits vor der Fortbildung recht hohen Wissensstand. Dieser Effekt gilt sowohl kurzfristig (t0 zu t1) als auch mittelfristig (t0 zu t2).

Ebenfalls auf Ebene der individuellen Eigenschaften zeigt sich für Personen mit überdurchschnittlicher Berufserfahrung ein signifikant geringerer kurzfristiger Fortbildungseffekt für fachdidaktisches Wissen. Der Befund deutet darauf hin, dass Personen mit geringer Berufserfahrung einen größeren Lernerfolg auf Wissensebene zeigen und kurzfristig stärker von der Fortbildungsteilnahme profitieren als sehr erfahrene Personen. Dieser Zusammenhang zeigt sich mittelfristig allerdings nicht mehr. Die Ergebnisse zum Kompetenzausgangsniveau und zur Berufserfahrung legen nahe, dass Pädagoginnen und Pädagogen, die ihr Wissen vorab bereits höher einschätzen, in der Fortbildung weniger Möglichkeiten haben, sich in Hinblick auf diese Kompetenz noch stark zu entwickeln.

Weiterhin wird der Zusammenhang von organisationalen Rahmenbedingungen auf den kurz- und mittelfristigen Fortbildungseffekt untersucht. Hier zeigt sich, dass der MINT-bezogene Betreuungsschlüssel eine wichtige Rolle zu spielen scheint. Personen, die in Einrichtungen tätig sind, in denen der MINT-bezogene Betreuungsschlüssel höher als der Durchschnitt ist, haben einen signifikant höheren mittelfristigen Zuwachs (t0 zu t2) im fachdidaktischen Wissen. Hiermit weist ein Merkmal, das die Personalressourcen in der Einrichtung beschreibt, unter denen MINT-bezogene Interaktionen im pädagogischen Alltag durchgeführt werden können, einen positiven Zusammenhang mit dem fachdidaktischen Wissen auf. Dies deutet darauf hin, dass die organisationalen Rahmenbedingungen in den Einrichtungen (siehe die Ausführungen in Abschnitt B 3.3 zum Zusammenhang von MINT-bezogener Betreuungsschlüssel und Teilnahmeeffekten für Motivation und Selbstwirksamkeit) eine wichtige Rolle bei der dauerhaften Verstetigung des Wissens und der Wissensgenerierung spielen. Das Ergebnis der Zusammenhangsanalysen lässt vermuten, dass ein guter MINT-bezogener Betreuungsschlüssel dazu beiträgt, dass Fortbildungsinhalte im pädagogischen Alltag auch angewendet und geübt werden können.

Die Zusammenhänge zwischen der Entwicklung des fachdidaktischen Wissens nach einem Fortbildungsbesuch und dem Wissensstand zu to sind als starke Effekte einzuordnen (Cohens $f^{2}=0.59$ bis 0.71 , vgl. Abschnitt B 2.4), während die übrigen berichteten signifikanten Zusammenhänge als geringfügig bis klein einzuordnen sind (Cohens $f^{2}<0.01$ bis 0.05 ).

Hinsichtlich der anderen individuellen und organisationalen Faktoren ergeben sich keine signifikanten Zusammenhänge zur Wissensentwicklung nach der Fortbildung. 


\section{Motivation in Bezug auf das Entdecken und Forschen}

Im Folgenden werden die Zusammenhänge zwischen den Wirkungen der Fortbildungsteilnahme auf die Motivation in Bezug auf das Entdecken und Forschen mit Kindern mit den individuellen Eigenschaften und organisationalen Rahmenbedingungen betrachtet (siehe Tabelle 34).

Tabelle 34. Ergebnisse der Regressionsanalysen zum Zusammenhang zwischen den Wirkungen der Fortbildungsteilnahme auf die Motivation und individuellen Eigenschaften sowie organisationalen Rahmenbedingungen ( $N=295-316)$

\begin{tabular}{lcc}
\hline Konstante & $\begin{array}{c}\text { Kurzfristiger } \\
\text { Effekt (t0 zu t1) }\end{array}$ & $\begin{array}{c}\text { Mittelfristiger } \\
\text { Effekt (t0 zu t2) }\end{array}$ \\
\hline Fortbildungsanzahl vor t0 & $\mathbf{0 . 2 3}^{\star *}$ & 0.08 \\
\hline Fortbildungsanzahl zwischen t1 und t2 & 0.00 & 0.01 \\
Fortbildungsfrequenz zu t0 & - & 0.04 \\
Motivationsstand zu t0 (z) & 0.00 & 0.05 \\
Berufserfahrung (z) & $-\mathbf{0 . 4 4 ^ { \star * }}$ & $-0.60^{\star *}$ \\
Bildungsabschluss (Studium) & -0.00 & 0.00 \\
\hline Funktion (Lehrkraft) & 0.08 & 0.12 \\
Funktion (Leitung) & -0.11 & $-0.23^{\star}$ \\
MINT-Bildungsinhalte in Konzeption & -0.14 & 0.04 \\
Betreuungsschlüssel (z) & - & 0.07 \\
MINT-bezogener Betreuungsschlüssel (z) & - & -0.52 \\
\hline Korrigiertes $\mathrm{R}^{2}$ & - & $1.37^{\star}$ \\
\hline Annekung: Dargetlt & 0.28 & 0.34 \\
\hline
\end{tabular}

Anmerkung: Dargestellt sind unstandardisierte Regressionskoeffizienten $(B) ;{ }^{\star \star} p<.05$; ${ }^{*} p$ <. 10; t0: vor der Fortbildung; t1: direkt nach der Fortbildung; $\mathrm{t} 2: 2-5$ Monate nach der Fortbildung; z: Variable zentriert.

Zunächst wird untersucht, ob für Personen mit verschiedenen individuellen Merkmalen Unterschiede in der Wirksamkeit des Fortbildungsbesuchs bestehen. Hier zeigt sich, dass Personen mit einem überdurchschnittlichen selbsteingeschätzten Motivationsstand zu to für die beiden betrachteten Zeiträume signifikant geringer von einem Fortbildungsbesuch zu profitieren scheinen. Je höher Personen ihre Motivation vor einer Fortbildung einschätzen, umso geringer fällt ihr Motivationszuwachs nach einem einzelnen Fortbildungsbesuch aus. Im Umkehrschluss deutet dieser Befund darauf hin, dass Personen mit einem geringeren selbsteingeschätzten Ausgangsniveau für Motivation in Bezug auf das Entdecken und Forschen mit Kindern ihre Motivation nach einem Fortbildungsbesuch höher einschätzen. Dieser Effekt gilt sowohl kurzfristig (t0 zu t1) als auch noch stärker mittelfristig (t0 zu t2). Wie schon für das fachdidaktische Wissen berichtet, scheinen auch hinsichtlich der Entwicklung der Motivation Personen von einem einzelnen 
Fortbildungsbesuch zu profitieren, die zuvor weniger motiviert waren, mit Kindern zu entdecken und zu forschen.

Zum Zusammenhang der organisationalen Rahmenbedingungen mit der Wirksamkeit des Fortbildungsbesuchs auf die Motivation zeigt sich ein signifikanter Unterschied hinsichtlich der beruflichen Funktion. Lehrkräfte scheinen mittelfristig (t0 zu t2) hinsichtlich ihrer Motivation etwas weniger von einem einzelnen Fortbildungsbesuch zu profitieren als pädagogische Fachkräfte. Dies könnte darauf zurückzuführen sein, dass die Inhalte der hier betrachteten Präsenzfortbildungen eher am Alltag von pädagogischen Fachkräften in Kita, Hort und Ganztag orientiert sind und deren Anwendung im Grundschulunterricht schwererfällt.

Weiterhin scheinen Personen, die in Einrichtungen tätig sind, in denen der MINT-bezogene Betreuungsschlüssel überdurchschnittlich ist, mittelfristig (t0 zu t2) hinsichtlich ihrer Motivation signifikant mehr von einem einzelnen Fortbildungsbesuch zu profitieren. Je besser also die personellen Ressourcen in der Einrichtung für MINT-spezifische Interaktionen sind, desto höher scheint der mittelfristige Zuwachs in der Motivation nach einem einzelnen Fortbildungsbesuch zu sein. Dieser Befund zeigt sich ebenfalls für das fachdidaktische Wissen und bestärkt die Annahme, dass die organisationalen Rahmenbedingungen in der Einrichtung eine wichtige Rolle für die dauerhafte Kompetenzentwicklung spielen.

Die Zusammenhänge zwischen der Entwicklung der Motivation nach einem Fortbildungsbesuch und dem Motivationsstand zu to sind als starke Effekte einzuordnen (Cohens $f^{2}=0.36$ bis 0.51 ), während die übrigen berichteten signifikanten Zusammenhänge als geringfügige Effekte einzuordnen sind (Cohens $f^{2}=0.01$ ).

Hinsichtlich der anderen individuellen und organisationalen Faktoren ergaben sich keine signifikanten Zusammenhänge zur Motivationsentwicklung nach der Fortbildung.

\section{Selbstwirksamkeit in Bezug auf das Entdecken und Forschen}

Schließlich werden die Ergebnisse der Regressionsanalysen zum Zusammenhang zwischen individuellen Eigenschaften sowie organisationalen Rahmenbedingungen und den Wirkungen der Fortbildungsteilnahme auf die Selbstwirksamkeit in Bezug auf das Entdecken und Forschen mit Kindern betrachtet (siehe Tabelle 35). 
Tabelle 35. Ergebnisse der Regressionsanalysen zum Zusammenhang zwischen den Wirkungen der Fortbildungsteilnahme auf die Selbstwirksamkeit und individuellen sowie organisationalen Rahmenbedingungen ( $N=292-310)$

\begin{tabular}{|c|c|c|}
\hline & $\begin{array}{c}\text { Kurzfristiger } \\
\text { Effekt (t0 zu t1) }\end{array}$ & $\begin{array}{c}\text { Mittelfristiger } \\
\text { Effekt (to zu t2) }\end{array}$ \\
\hline Konstante & $0.33^{* *}$ & 0.15 \\
\hline Fortbildungsanzahl vor t0 & $0.02^{\star}$ & 0.02 \\
\hline Fortbildungsanzahl zwischen $\mathrm{t} 1$ und $\mathrm{t} 2$ & - & 0.04 \\
\hline Fortbildungsfrequenz zu to & -0.06 & 0.04 \\
\hline Selbstwirksamkeitsstand zu to (z) & $-0.58^{* *}$ & $-0.66 * *$ \\
\hline Berufserfahrung (z) & -0.01 ** & -0.00 \\
\hline Bildungsabschluss (Studium) & 0.06 & -0.02 \\
\hline Funktion (Lehrkraft) & -0.03 & -0.19 \\
\hline Funktion (Leitung) & 0.04 & 0.02 \\
\hline MINT-Bildungsinhalte in Konzeption & - & -0.03 \\
\hline Betreuungsschlüssel (z) & - & -0.89 \\
\hline MINT-bezogener Betreuungsschlüssel (z) & - & $1.97^{\star \star}$ \\
\hline Korrigiertes $\mathrm{R}^{2}$ & 0.42 & 0.39 \\
\hline
\end{tabular}

Anmerkung: Dargestellt sind unstandardisierte Regressionskoeffizienten $(B) ;{ }^{* \star} p<.05$; ${ }^{*} p$ < .10; t0: vor der Fortbildung; t1: direkt nach der Fortbildung; t2: 2-5 Monate nach der Fortbildung; z: Variable zentriert.

In Hinblick auf die individuellen Eigenschaften zeigt sich ein Zusammenhang zwischen der Fortbildungsanzahl und dem kurzfristigen Fortbildungseffekt. Personen mit höherer Anzahl besuchter Fortbildungen vor t0 weisen direkt nach der Fortbildung eine signifikant höhere Selbstwirksamkeit auf (t0 zu t1). Kurzfristig betrachtet profitieren also Personen in ihrer Selbstwirksamkeitsentwicklung umso mehr von der Teilnahme an einer einzelnen Fortbildung, je mehr Fortbildungen sie insgesamt bereits besucht haben. Dieser Zusammenhang zeigt sich mittelfristig allerdings nicht mehr.

Zudem zeigt sich, wie schon für die anderen Kompetenzen, ein Zusammenhang mit dem Ausgangsniveau der selbsteingeschätzten Selbstwirksamkeit zum Messzeitpunkt t0. Personen mit einem unterdurchschnittlichen selbsteingeschätzten Selbstwirksamkeitsstand zu to scheinen in beiden betrachteten Zeiträumen mehr von einem Fortbildungsbesuch zu profitieren. Im Umkehrschluss deutet dieser Befund darauf hin, dass Personen mit einem höheren Ausgangsniveau ihrer Selbstwirksamkeit in Bezug auf das Entdecken und Forschen mit Kindern zu to weniger von einem Fortbildungsbesuch profitieren als Personen mit einem bereits vor der Fortbildung recht niedrigen Selbstwirksamkeitsstand. Dieser Effekt gilt sowohl kurzfristig (t0 zu t1) als auch mittelfristig (t0 zu t2). 
Ein weiterer Unterschied im Fortbildungseffekt zeigt sich hinsichtlich der Berufserfahrung. Für Personen mit überdurchschnittlicher Berufserfahrung fällt der kurzfristige Zuwachs in der Selbstwirksamkeit nach einem einzelnen Fortbildungsbesuch geringer aus. Hinsichtlich der kurzfristigen Entwicklung der Selbstwirksamkeit profitieren also weniger erfahrene Personen. Dieser Zusammenhang zeigt sich mittelfristig allerdings nicht mehr.

Weiterhin wird der Frage nachgegangen, ob Zusammenhänge organisationaler Rahmenbedingungen mit der Wirksamkeit eines Fortbildungsbesuchs bestehen. Hierzu kann - wie auch schon bei den anderen Kompetenzen - festgestellt werden, dass Personen, die in Einrichtungen tätig sind, in denen der MINT-bezogene Betreuungsschlüssel überdurchschnittlich ist, mittelfristig einen signifikant höheren Zuwachs in der Selbstwirksamkeit aufzuweisen scheinen. Je besser die personellen Ressourcen in der Einrichtung für MINT-spezifische Interaktionen sind, desto höher ist der mittelfristige Zuwachs in der Selbstwirksamkeit der Personen nach einem einzelnen Fortbildungsbesuch. Dieser Befund spricht ebenfalls dafür, dass organisationale Rahmenbedingungen in der Einrichtung eine wichtige Rolle für die dauerhafte Kompetenzentwicklung spielen.

Die Zusammenhänge zwischen der Entwicklung der Selbstwirksamkeit nach einem Fortbildungsbesuch und dem Selbstwirksamkeitsstand zu t 0 sind als große Effekte einzuordnen (Cohens $f^{2}=0.63$ bis 0.68 ), während die übrigen berichteten signifikanten Zusammenhänge als geringfügige bis kleine Effekte einzuordnen sind (Cohens $f^{2}=0.01$ bis 0.05 ).

Hinsichtlich der anderen individuellen und organisationalen Faktoren ergaben sich keine signifikanten Zusammenhänge zur Selbstwirksamkeitsentwicklung nach der Fortbildung.

\section{Zusammenfassung und Diskussion}

In den dargestellten Analysen wurde untersucht, ob es personenspezifische Unterschiede im Lernerfolg gibt und welche individuellen Eigenschaften und organisationalen Rahmenbedingungen in Zusammenhang mit den Wirkungen der Fortbildungsteilnahme stehen.

Auf Ebene der individuellen Eigenschaften scheint das selbsteingeschätzte Kompetenzniveau der Pädagoginnen und Pädagogen zum Zeitpunkt vor der Fortbildung (t0) den größten Zusammenhang mit den Fortbildungseffekten zu haben. Personen, die ihr fachdidaktisches Wissen, ihre Motivation beziehungsweise ihre Selbstwirksamkeit in Bezug auf das Entdecken und Forschen mit Kindern zu Beginn geringer einschätzen, scheinen am meisten von einer einzelnen Fortbildung in der mittelfristigen Entwicklung dieser Kompetenzen zu profitieren. Personen, die ihre Kompetenzen bereits zu Beginn höher einschätzen, verbleiben auf ihrem Ausgangsniveau. Dies kann, wie bereits in Abschnitt B 3.1. beschrieben, auch 
daran liegen, dass es für diese Personen einen Deckeneffekt gibt und die Erfassung der Kompetenzentwicklung mit den hier genutzten Instrumenten begrenzt ist. Wenn das Ausgangsniveau bereits sehr hoch ist, gibt es eventuell weniger Möglichkeiten, sich zu entwickeln.

Entsprechend zu diesem Befund scheinen Personen mit weniger Berufserfahrung kurzfristig stärker von einem einzelnen Fortbildungsbesuch hinsichtlich der Entwicklung ihres fachdidaktischen Wissens und ihrer Selbstwirksamkeit zu profitieren. Dies könnte damit erklärt werden, dass berufsunerfahrenere Personen ihre Kompetenzen geringer einschätzen als erfahrene Personen. Andererseits könnte es sein, dass die Fortbildungen wenig Differenzierung für Personen mit höherem Ausgangsniveau und Erfahrung ermöglichen.

Personen, die vorab bereits mehr Fortbildungen besucht haben, zeigen kurzfristig eine höhere Selbstwirksamkeit und mittelfristig ein höheres selbsteingeschätztes fachdidaktisches Wissen. Dieser Effekt ist jedoch relativ klein, was zu der Annahme führt, dass die Kompetenzentwicklung nach einem einzelnen Fortbildungsbesuch unter Konstanthaltung des Kompetenzniveaus (zu to) eher unabhängig davon ist, wie viele Fortbildungen zuvor bereits besucht wurden. Hier besteht die Vermutung, dass die Anzahl besuchter „Haus der kleinen Forscher“Fortbildungen eher indirekt in Zusammenhang mit der Kompetenzentwicklung stehen könnte, sodass sich bei gleichzeitiger Betrachtung von Fortbildungsanzahl (zu t0) und Kompetenzniveau (zu t0) vor allem ein Zusammenhang des Kompetenzniveaus mit den Fortbildungseffekten zeigt. Ein möglicher Zusammenhang zwischen Fortbildungsanzahl und Kompetenzniveau wird in Abschnitt B 3.3 untersucht.

In Hinblick auf den Zusammenhang von Fortbildungseffekten mit den organisationalen Rahmenbedingungen scheint insbesondere der MINT-bezogene Betreuungsschlüssel eine wichtige Rolle zu spielen. Sowohl für die mittelfristige Entwicklung des fachdidaktischen Wissens als auch der Motivation und der Selbstwirksamkeit zeigt sich, dass ein besserer MINT-bezogener Betreuungsschlüssel positiv mit der Kompetenzentwicklung zusammenhängt. Das sich hier abzeichnende Muster weist auf die Bedeutung der organisationalen Rahmenbedingungen in den Einrichtungen für die dauerhafte Verstetigung von Kompetenzen hin.

Die berufliche Funktion der Pädagoginnen und Pädagogen scheint hingegen in keinem praktisch bedeutsamen Zusammenhang damit zu stehen, wie sich ein Fortbildungsbesuch kurz- und mittelfristig auf die Kompetenzentwicklung auswirkt. Pädagogische Fachkräfte, Lehrkräfte und Leitungskräfte unterscheiden sich in ihrer Kompetenzentwicklung nicht bedeutsam voneinander. 


\subsection{Zusammenhänge der Fortbildungsanzahl und -frequenz mit dem Ausgangsniveau verschiedener Kompetenzen}

Nachdem zuvor die Fortbildungseffekte auf die kurz- und mittelfristige Kompetenzentwicklung, auch in Hinblick auf individuelle Eigenschaften und organisationale Rahmenbedingungen untersucht wurden, werden in diesem Abschnitt Effekte einer mehrfachen Fortbildungsteilnahme über einen längeren Zeitraum betrachtet. Im Fokus steht die Frage, ob die Fortbildungsanzahl und -frequenz vor dem betrachteten Fortbildungsbesuch (t0) in Zusammenhang mit dem Kompetenzniveau der befragten Pädagoginnen und Pädagogen zum Messzeitpunkt t0 stehen (Fragestellung 3). Des Weiteren soll untersucht werden, ob weitere individuelle Eigenschaften oder organisationale Rahmenbedingungen in Zusammenhang mit dem Kompetenzausgangsniveau der Befragten stehen.

Bei der Analyse kommen Verfahren der multiplen Regression zur Anwendung. Für jede der drei Kompetenzen fachdidaktisches Wissen, Motivation und Selbstwirksamkeit in Bezug auf das Entdecken und Forschen mit Kindern wird ein regressionsanalytisches Modell berechnet. Es ist dabei zu beachten, dass die Ergebnisse nur retrospektiv Hinweise auf mögliche längerfristige Fortbildungseffekte liefern können, da die Längsschnittbefragung prospektiv noch nicht ausreichend weit fortgeschritten ist. Neben der Fortbildungsanzahl und -frequenz wird für $\mathrm{Zu}$ sammenhänge mit den in Abschnitt B 2.2 beschriebenen weiteren Faktoren auf individueller und organisationaler Ebene kontrolliert.

Die in Tabelle 36 dargestellten Regressionsparameter $(B)$ geben Hinweise auf mögliche längerfristige Effekte der Fortbildungsteilnahme durch die Fortbildungsanzahl und -frequenz sowie den Zusammenhang zwischen den erläuterten individuellen und organisationalen Merkmalen und dem Kompetenzniveau zum Messzeitpunkt t0. 
Tabelle 36. Ergebnisse der Regressionsanalysen zum Zusammenhang zwischen dem Kompetenzniveau zum ersten Messzeitpunkt und individuellen sowie organisationalen Faktoren $(N=297-304)$

\begin{tabular}{|c|c|c|c|}
\hline & $\begin{array}{l}\text { Fachdidakti- } \\
\text { sches Wissen } \\
\text { (to) }\end{array}$ & $\begin{array}{l}\text { Motivation } \\
\text { (t0) }\end{array}$ & $\begin{array}{l}\text { Selbstwirk- } \\
\text { samkeit (t0) }\end{array}$ \\
\hline Konstante & $4.02^{\star \star}$ & $4.67^{\star \star}$ & $4.56 * \star$ \\
\hline Fortbildungsanzahl vor to & $0.06 * *$ & 0.04 ** & $0.04^{\star \star}$ \\
\hline Fortbildungsfrequenz vor to & $0.18^{\star *}$ & 0.03 & 0.08 \\
\hline Berufserfahrung (z) & -0.00 & $-0.01 *$ & -0.00 \\
\hline Bildungsabschluss (Studium) & -0.01 & -0.07 & -0.05 \\
\hline Funktion (Lehrkraft) & -0.27 & -0.04 & -0.03 \\
\hline Funktion (Leitung) & -0.02 & 0.06 & -0.01 \\
\hline MINT-Bildungsinhalte in Konzeption & 0.11 & $0.20^{\star}$ & 0.07 \\
\hline Betreuungsschlüssel (z) & 0.77 & 1.09 & $1.37^{\star}$ \\
\hline MINT-bezogener Betreuungsschlüssel (z) & 0.13 & -1.44 & $-1.85^{\star}$ \\
\hline Korrigiertes $\mathrm{R}^{2}$ & 0.16 & 0.06 & 0.05 \\
\hline
\end{tabular}

Anmerkung: Dargestellt sind unstandardisierte Regressionskoeffizienten $(B) ;{ }^{\star \star} p<.05$; ${ }^{*} p<.10$; t0: vor der Fortbildung; $z$ : Variable zentriert.

\section{Zusammenhänge mit individuellen Eigenschaften}

Mit Blick auf die Wirksamkeit der Fortbildungsteilnahme ist von besonderem Interesse, inwieweit die Fortbildungsanzahl und -frequenz in Zusammenhang mit dem selbsteingeschätzten Kompetenzausgangsniveau stehen, wenn für den Einfluss aller weiteren Merkmale in der Analyse kontrolliert wird. Wie in Tabelle 36 dargestellt, zeigt sich ein positiver Zusammenhang der Fortbildungsanzahl mit dem selbsteingeschätzten Ausgangsniveau. Mit jeder zusätzlich besuchten Fortbildung steigt die selbsteingeschätzte Kompetenz für alle drei Kompetenzen an.

Im Gegensatz zur Fortbildungsanzahl zeigt sich für die Fortbildungsfrequenz lediglich ein signifikanter Zusammenhang mit dem Ausgangsniveau des fachdidaktischen Wissens. Mit jeder weiteren Fortbildung pro Jahr erhöht sich das selbsteingeschätzte fachdidaktische Wissen. Der Zusammenhang der Fortbildungsfrequenz mit dem fachdidaktischen Wissen ist im Vergleich zur Fortbildungsanzahl hierbei größer.

Für die Motivation kann auf Ebene der individuellen Eigenschaften neben der Fortbildungsanzahl lediglich ein weiterer Zusammenhang mit der Berufserfahrung beobachtet werden. Mit jedem zusätzlichen Jahr Berufserfahrung zeigt sich eine leichte Reduktion in der selbsteingeschätzten Motivation vor Fortbildungsbeginn. Dies deutet im Umkehrkehrschluss darauf hin, dass weniger berufserfahrene Pädagoginnen und Pädagogen vor der Fortbildung eine höhere Motivation für das Entdecken und Forschen mit Kindern zeigen. 
Die Zusammenhänge zwischen dem Kompetenzniveau und den individuellen Eigenschaften sind als geringfügige bis kleine Effekte einzuordnen (Cohens $f^{2}<0.01$ bis 0.04 , vgl. Abschnitt B 2.4).

Hinsichtlich des höchsten Bildungsabschlusses ergaben sich keine signifikanten Zusammenhänge zum Kompetenzausgangsniveau.

\section{Zusammenhänge mit organisationalen Rahmenbedingungen}

Auf Ebene der organisationalen Rahmenbedingungen ergeben sich nur für die Kompetenzen Motivation und Selbstwirksamkeit signifikante Zusammenhänge. So zeigt sich etwa für Pädagoginnen und Pädagogen aus Einrichtungen mit MINTBildungsinhalten in der Einrichtungskonzeption ein signifikant erhöhtes Motivationsniveau zum Messzeitpunkt t0. Die Selbstbeschreibung der Organisation als MINT-Einrichtung sowohl in der Innen- als auch Außendarstellung scheint somit in positivem Zusammenhang mit dem Motivationsniveau der Pädagoginnen und Pädagogen zu stehen.

Für die Selbstwirksamkeit zeigen sich mit Bezug auf die organisationalen Rahmenbedingungen gegensätzliche Zusammenhänge. Zum einen gibt es einen positiven Zusammenhang mit dem allgemeinen Betreuungsschlüssel, zum anderen jedoch einen negativen Zusammenhang mit dem MINT-bezogenen Betreuungsschlüssel. Dies bedeutet, dass ein höherer allgemeiner Betreuungsschlüssel, d. $h$. potenziell mehr Zeit pro Kind für Interaktionen aller Art, mit einer erhöhten Selbstwirksamkeitswahrnehmung für das Entdecken und Forschen einhergeht. Ein höherer MINT-bezogener Betreuungsschlüssel geht hingegen mit einer geringeren Selbstwirksamkeitswahrnehmung einher.

Die Zusammenhänge zwischen dem Kompetenzniveau und den organisationalen Rahmenbedingungen sind als geringfügige Effekte einzuordnen (Cohens $f^{2}$ <0.02).

Hinsichtlich der beruflichen Funktion in der Einrichtung ergaben sich keine signifikanten Zusammenhänge zum Kompetenzausgangsniveau.

\section{Zusammenfassung und Diskussion}

In den hier berichteten Analysen wurde untersucht, ob die Fortbildungsanzahl und -frequenz vor dem betrachteten Fortbildungsbesuch (t0) sowie weitere individuelle Eigenschaften und organisationale Rahmenbedingungen in Zusammenhang mit dem Kompetenzausgangsniveau der Pädagoginnen und Pädagogen stehen.

Die Ergebnisse zeigen einen Zusammenhang mit der Anzahl zuvor besuchter „Haus der kleinen Forscher"-Fortbildungen für alle drei Kompetenzen fachdidaktisches Wissen, Motivation sowie Selbstwirksamkeit in Bezug auf das Entdecken und Forschen mit Kindern. Haben die Pädagoginnen und Pädagogen bereits mehr Fortbildungen besucht, so schätzen sie das Ausgangsniveau der drei Kompeten- 
zen höher ein. Für das fachdidaktische Wissen scheint die Fortbildungsfrequenz eine noch größere Rolle zu spielen. Personen mit höherer Fortbildungsfrequenz, also mehr besuchten Fortbildungen pro Jahr, schätzen ihre fachdidaktische Kompetenz hier zu Beginn höher ein.

Das Ausgangsniveau von Motivation und Selbstwirksamkeit scheint zudem in Zusammenhang mit organisationalen Rahmenbedingungen wie der Verankerung von MINT-Bildungsinhalten in der Einrichtungskonzeption und dem allgemeinen Betreuungsschlüssel zu stehen. Ein höherer MINT-bezogener Betreuungsschlüssel geht hingegen mit einer geringeren Selbstwirksamkeitswahrnehmung einher. Es ist möglich, dass es weitere bedeutsame Organisationsmerkmale gibt, welche in Beziehung zur Selbstwirksamkeitsentwicklung beim Entdecken und Forschen stehen, jedoch im Rahmen dieser Studie bisher nicht erhoben wurden.

Bei der Interpretation der Ergebnisse ist zu beachten, dass die hier erhobenen individuellen Eigenschaften und organisationalen Rahmenbedingungen jeweils nur einen geringen Teil der Unterschiede in den selbsteingeschätzten Kompetenzniveaus erklären. 


\section{Allgemeine Diskussion}

\subsection{Methodische Grenzen der Analysen}

Aufgrund des angewandten Untersuchungs- und Analysedesigns können die dargestellten Zusammenhänge nicht als kausale Effekte der Fortbildungsteilnahme auf die Kompetenzentwicklung interpretiert werden, d. h., die Entwicklungen können auch aufgrund anderer Faktoren stattgefunden haben. Da in dem vorliegenden Untersuchungsdesign kein Kontrollgruppendesign zur Anwendung kommt, können keine Aussagen über die Kompetenzentwicklung für die befragten Pädagoginnen und Pädagogen im Vergleich zu nicht am Fortbildungsprogramm teilnehmenden Pädagoginnen und Pädagogen getroffen werden. Dennoch können durch das Prä-Post-Design jedoch zum Teil bedeutsame Effekte des Fortbildungsbesuchs unter Berücksichtigung von Kontrollvariablen identifiziert werden.

Die Güte der Regressionsmodelle ist, wie dargestellt, für die kurz- und mittelfristigen Fortbildungseffekte (siehe Abschnitt B 3.2) aller Kompetenzen zufriedenstellend. Die Modelle klären demnach einen großen Anteil der Varianz beziehungsweise der Unterschiede in den Fortbildungseffekten für Personen mit verschiedenen individuellen Eigenschaften und organisationalen Rahmenbedingungen auf. Da sich aber die Güte der Modelle für Zusammenhänge mit dem Kompetenzausgangsniveau (siehe Abschnitt B 3.3) gering darstellt, ist zu vermuten, dass weitere mit der Kompetenzentwicklung von Pädagoginnen und Pädagogen in Zusammenhang stehende Merkmale auf individueller und organisationaler Ebene (z. B. Variablen zum Teamklima) bisher nicht erfasst wurden.

Auch wenn Aussagen zur Selbsteinschätzung in der empirischen Sozialforschung weit verbreitet sind, heften diesen Erhebungsinstrumenten methodische Mängel an (vgl. Bortz \& Döring, 2006). Darüber hinaus sind in den hier beschriebenen Ergebnissen Verzerrungen der Wahrnehmung des eigenen Kompetenzniveaus möglich, je nachdem zu welchem Zeitpunkt nach einem Fortbildungsbesuch die Selbsteinschätzung vorgenommen wird. Um Kompetenzen mit möglichst wenig Verzerrung von Urteilsfehlern zu erfassen, werden in der Längsschnittbefragung auch situationsspezifische Vignetten zur Erfassung von alltagsnahem Verhalten eingesetzt. Diese werden in weiteren Analysen ausgewertet und berichtet. Ergebnisse und Diskussion zur Erfassung der selbst berichteten beziehungsweise beobachteten Prozessqualität des Forschens im Zusammenhang mit der Teilnahme am „Haus der kleinen Forscher“-Fortbildung- beziehungsweise am Zertifizierungsprogramm finden sich etwa bei Anders und Ballaschk (Anders \& Ballaschk, 2014) oder im Monitoring-Bericht 2016/2017 (vgl. Stiftung Haus der kleinen For- 
scher, 2017) sowie im Monitoring-Bericht 2013 (Stiftung Haus der kleinen Forscher, 2013).

Eine weitere methodische Einschränkung bei Längsschnittbefragungen stellt der sogenannte Paneleffekt dar. Dies meint eine mögliche Veränderung des Antwortverhaltens der Befragten durch die wiederholte Befragung selbst (vgl. Schupp, 2014). Inwiefern die Datenqualität dadurch beeinflusst wird, ist unklar. Darüber hinaus tritt auch bei dieser Untersuchung mit dem Verlust von an der Befragung Teilnehmenden (sogenannten Panelmortalität) ein typisches Problem von Längsschnittbefragungen auf (vgl. Rendtel, 1995). Bei einem Verlust von ca. zwei Dritteln der an der Ersterhebung (t0/t1) befragten Pädagoginnen und Pädagogen im Vergleich zur Folgeerhebung (t2) stellt sich zudem die Frage, ob es sich dabei um zufällige oder systematische Ausfälle handelt. Eine Dropout-Analyse zeigt, dass die in den dargestellten Analysen betrachtete Stichprobe im Vergleich zu den nach der Ersterhebung (t0/t1) Ausgeschiedenen leicht motivierter ist und sich tendenziell selbstwirksamer einschätzt, mit Kindern zu entdecken und zu forschen. Der Ausfall von Pädagoginnen und Pädagogen, die sich in den betrachteten Kompetenzen geringer einschätzen, könnte die Ergebnisse (z. B. aufgrund einer geringeren Varianz) verzerren. Um der Panelmortalität entgegenzuwirken und den Rücklauf zu erhöhen, ist künftig neben Online-Erhebungen ein ergänzender Einsatz von Telefonbefragungen geplant.

Die in Abschnitt B 3.1 gezeigten Ergebnisse stellen durchschnittliche Effekte dar, die personenspezifische Unterschiede nicht berücksichtigen und daher möglicherweise durch die in Abschnitt B 2.3 beschriebenen Eigenschaften der Stichprobe verzerrt sein könnten. So verfügt beispielsweise, wie beschrieben, ein überdurchschnittlich hoher Anteil der in der Längsschnittbefragung befragten Pädagoginnen und Pädagogen über einen Hochschulabschluss. Inwiefern dies den selbsteingeschätzten Lernerfolg nach einem Fortbildungsbesuch beeinflussen könnte, ist unklar.

\subsection{Gesamtzusammenfassung und Ausblick}

In einem Prä-Post-Design wurde mit Daten einer Längsschnittbefragung bei pädagogischen Fach-, Lehr- und Leitungskräften untersucht, welche kurzfristigen durchschnittlichen Effekte auf die Entwicklung verschiedener Kompetenzen es nach einem „Haus der kleinen Forscher“-Fortbildungsbesuch gibt und wie stabil diese Effekte mittelfristig über einige Monate sind. Zudem wurde untersucht, wie sich kurz- und mittelfristige Fortbildungseffekte in Abhängigkeit von individuellen Eigenschaften und organisationalen Rahmenbedingungen der Befragten unterscheiden. Schließlich wurde analysiert, welche Zusammenhänge es zwischen dem Kompetenzausgangsniveau und der Fortbildungsanzahl und -frequenz vor 


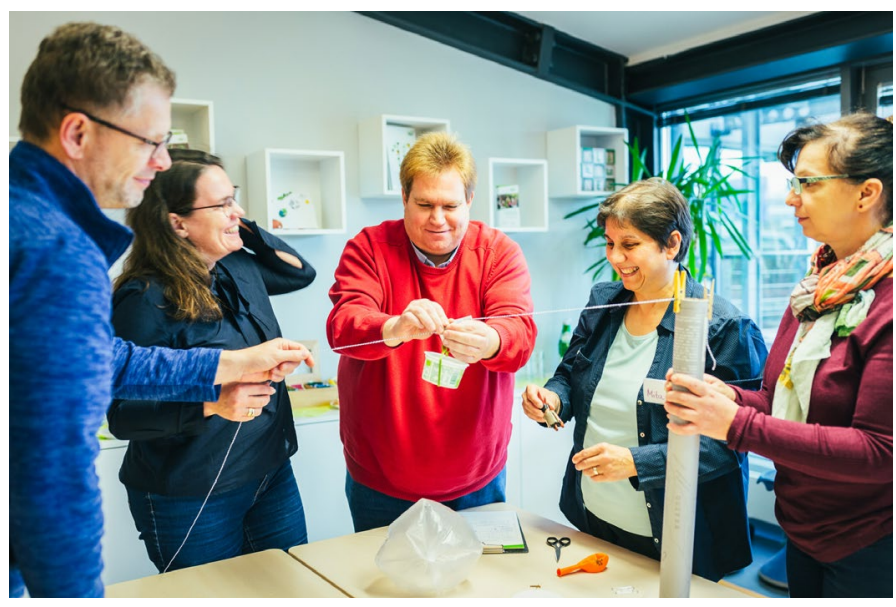

dem betrachteten Fortbildungsbesuch (t0) sowie weiteren Merkmalen auf individueller und organisationaler Ebene gibt.

Insgesamt zeigen sich bei den befragten Pädagoginnen und Pädagogen sowohl kurz- als auch mittelfristige Fortbildungseffekte auf die Kompetenzentwicklung nach dem Besuch einer „Haus der kleinen Forscher“Fortbildung. Das heißt, sie schätzen ihr fachdidaktisches Wissen sowohl direkt nach der Fortbildung als auch einige Monate später im Durchschnitt bedeutsam höher ein, empfinden sich als leicht motivierter, mit Kindern zu entdecken und zu forschen und trauen sich dies auch eher zu. Die beobachteten Kompetenzentwicklungseffekte (vor allem in Bezug auf fachdidaktisches Wissen) entsprechen den Zielen für die inhaltliche und didaktische Gestaltung der „Haus der kleinen Forscher“-Fortbildungen. Eine Erklärung für die berichteten geringeren Fortbildungseffekte auf Motivation und Selbstwirksamkeit könnte neben den hohen Ausgangswerten sein, dass eine Entwicklung dieser Kompetenzen erst längerfristig sichtbar wird. Daher plant die Stiftung, im Rahmen weiterer Erhebungen der Längsschnittbefragung Zeitreihenanalysen durchzuführen, um robuste Aussagen über die langfristige Kompetenzentwicklung von Pädagoginnen und Pädagogen treffen zu können.

Weiterhin wurde untersucht, ob es personenspezifische Unterschiede im Lernerfolg gibt und welche individuellen Eigenschaften und organisationalen Rahmenbedingungen in Zusammenhang mit der Effektivität der Fortbildungsteilnahme stehen. Insgesamt weisen die Ergebnisse darauf hin, dass Pädagoginnen und Pädagogen mit geringem Kompetenzausgangsniveau und wenig Berufserfahrung stärker von der Fortbildungsteilnahme profitieren. Für die Kompetenzentwicklung könnte es sich daher als förderlich erweisen, individuelle Unterschiede stärker zu berücksichtigen, z. B. nach unterschiedlichen Erfahrungsstufen differenzierte Fortbildungsformate zu entwickeln und anzubieten oder mehr Möglichkeiten zur Binnendifferenzierung zu integrieren, um den Pädagoginnen und Pädagogen die Möglichkeit zu bieten, sich je nach Kompetenzniveau bedarfsorientiert weiterentwickeln zu können. In Hinblick auf die Frage, welche pädagogischen Zielgruppen vom Besuch einer „Haus der kleinen Forscher“-Fortbildung profitieren, konnten keine Hinweise auf praktisch bedeutsame Unterschiede in der selbsteingeschätzten Kompetenzentwicklung zwischen pädagogischen Fach-, Lehr- und Leitungs- 
kräften gefunden werden. Aus den Ergebnissen kann gefolgert werden, dass die gezeigten Fortbildungseffekte für Pädagoginnen und Pädagogen in unterschiedlichen beruflichen Funktionen gleichermaßen gelten.

Auf Ebene der organisationalen Rahmenbedingungen scheint insbesondere der MINT-bezogene Betreuungsschlüssel eine Rolle zu spielen. Er stellt das Verhältnis der Anzahl aller Pädagoginnen und Pädagogen, die zu MINT-Bildungsinhalten entdecken und forschen, zur Anzahl der Kinder in der Einrichtung dar. Dieser hängt positiv mit der Entwicklung des fachdidaktischen Wissens, der Motivation und Selbstwirksamkeit zusammen. Gute organisationale Rahmenbedingungen in den Einrichtungen, beispielsweise durch einen entsprechenden Betreuungsschlüssel beziehungsweise ausreichend qualifizierte Fachkräfte, scheinen sehr bedeutsam für die dauerhafte Verstetigung von Kompetenzen zu sein.

Da mögliche mit der Kompetenzentwicklung von Pädagoginnen und Pädagogen in Zusammenhang stehende Merkmale auf individueller und organisationaler Ebene (z. B. Variablen zum Teamklima) bisher nicht erfasst wurden, sollen in künftigen Erhebungen der Längsschnittbefragung nach Möglichkeit weitere Faktoren erhoben und die Analyseverfahren entsprechend angepasst werden. Damit können perspektivisch umfassendere Aussagen darüber abgeleitet werden, wie organisationale Rahmenbedingungen und die Veränderung von pädagogischem Verhalten, angeregt durch Fortbildungsbesuche, miteinander zusammenhängen. Auch externe Begleitstudien und weitere Evaluationen zu den Wirkungen der Stiftungsarbeit setzen hier an und befassen sich insbesondere mit Veränderungen innerhalb der pädagogischen Einrichtung im Sinne einer Organisationsentwicklung (siehe Fazit und Ausblick, in diesem Band).

Zudem werden in den künftigen jährlich stattfindenden Erhebungen der Längsschnittbefragung höhere Fallzahlen als zur Folgebefragung t2 angestrebt, indem die Stichprobe einerseits um neue Teilnehmende ergänzt und der Fragebogenrücklauf andererseits durch den parallelen Einsatz verschiedener Erhebungsmethoden erhöht wird.

Die Qualität der Umsetzung von MINT-Bildung ist mit quantitativem Erhebungsdesign weniger leicht zu erfassen. Die Stiftung diskutiert hierzu im Austausch mit dem Wissenschaftlichen Beirat und weiteren Fachexpertinnen und -experten verschiedene Herangehensweisen mit quantitativen Messinstrumenten sowie mit qualitativen Methoden und verfolgt die Forschungsentwicklungen hierzu. Sie entwickelt Items zur Erfassung der Qualität von entdeckendem und forschendem Lernen mit Kindern weiter und setzt Vignetten zur Erfassung situationsspezifischer Verhaltensweisen ein.

Zusammengenommen zeigen die Ergebnisse, dass das jeweilige Kompetenzausgangsniveau der Pädagoginnen und Pädagogen den größten Zusammenhang mit den Fortbildungseffekten zeigt. Pädagoginnen und Pädagogen, die zuvor 
mehr Fortbildungen besucht haben, weisen ein höheres selbsteingeschätztes Ausgangsniveau sowohl für das fachdidaktische Wissen als auch für die Motivation und Selbstwirksamkeit, mit Kindern zu entdecken und zu forschen. Diese Ergebnisse sind Hinweise auf längerfristige Effekte der Fortbildungen.

Bei zukünftigen Erhebungen und Analysen wird weiter der Frage nachgegangen, auf welche Weise Rahmenbedingungen in der Einrichtung mit Veränderungsprozessen und dem Transfer der Fortbildungsinhalte zusammenhängen. Darüber hinaus soll die Bedeutung der Fortbildungsfrequenz weiter untersucht werden beispielsweise soll die Frage in den Blick genommen werden, welche Unterschiede in der Wirksamkeit von Fortbildungsformaten verschiedener Intensität bestehen. Für die (Weiter-)Entwicklung von Fortbildungsformaten empfiehlt es sich weiterhin, die individuellen und organisationalen Veränderungsprozesse mit Bezug zur Häufigkeit und Qualität der Umsetzung früher MINT-Bildung erfahrungs- und alltagsnah zu untersuchen, um so die erfolgsrelevanten Wirkungsmechanismen von Fortbildungsformaten zu rekonstruieren. 



\section{Fazit und Ausblick - Wie die Stiftung „Haus der kleinen Forscher" mit den Erkenntnissen umgeht}

Stiftung Haus der kleinen Forscher

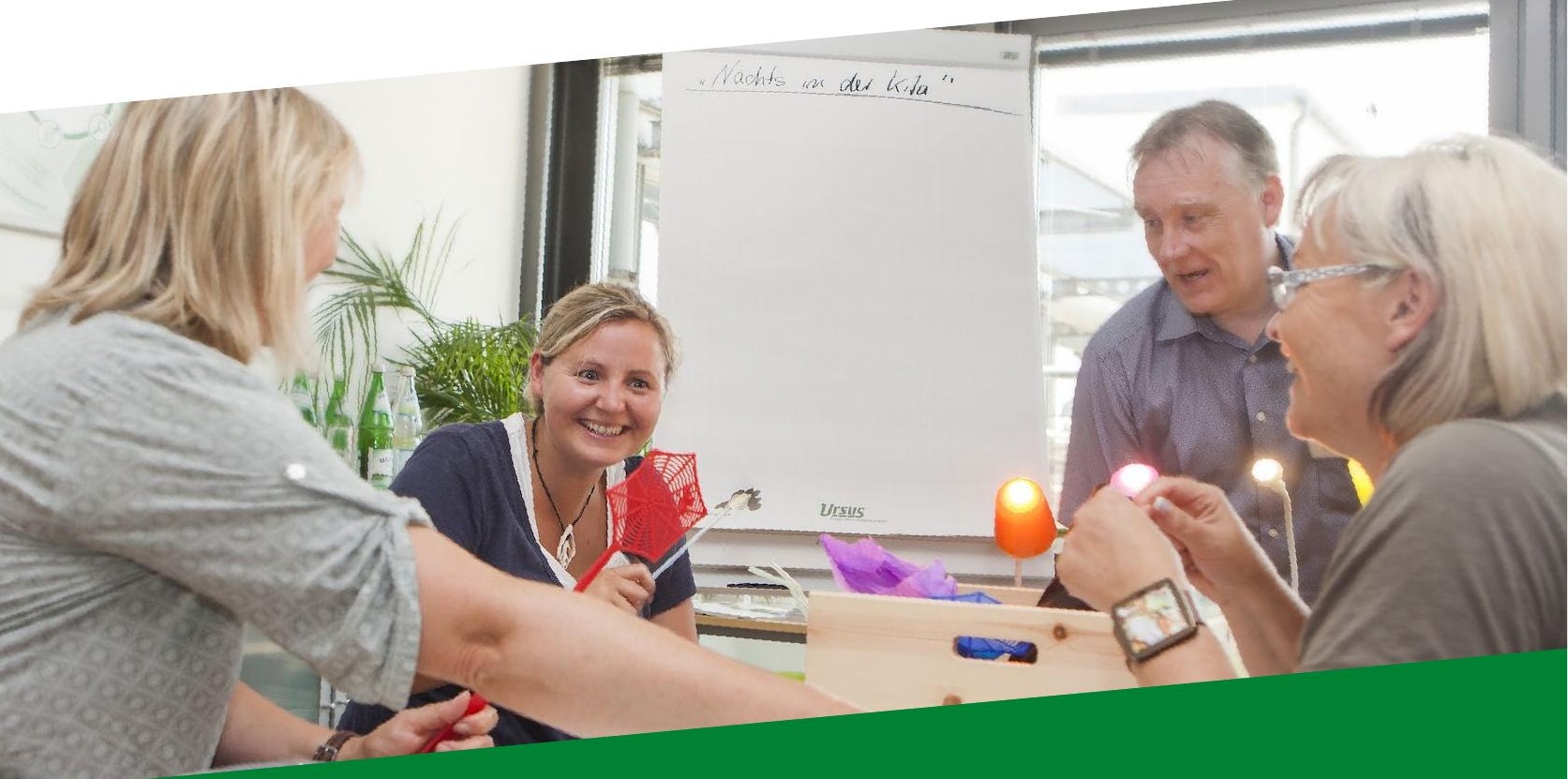

1 Ergebnisse der Untersuchungen und ihre Bedeutung für die Stiftungsarbeit

2 Grenzen der Untersuchungsergebnisse

3 Umgang mit den Empfehlungen und Erkenntnissen aus den Untersuchungen

4 Ausblick - weitere wissenschaftliche Begleitung und Erkenntnisbedarfe 


\section{Ergebnisse der Untersuchungen und ihre Bedeutung für die Stiftungsarbeit}

Die von der Stiftung und dem BMBF geförderte Studie „Entwicklungsverläufe von pädagogischen Fach- und Lehrkräften in der frühen MINT-Bildung“ („EpFL MINT“) untersuchte, wie sich die selbstwahrgenommenen Entwicklungsverläufe der pädagogischen Fach- und Lehrkräfte in Bezug auf die Professionalisierung im MINTBereich beschreiben lassen und ob verschiedene Phasen oder prägende Ereignisse eine besondere Rolle in ihrer professionellen Entwicklung spielen (siehe Beitrag A). Die Ergebnisse der explorativen Querschnittstudie sprechen dafür, dass die Anzahl besuchter Fortbildungen von zentraler Bedeutung für die professionelle Entwicklung pädagogischer Fach- und Lehrkräfte in der MINT-Bildung ist.

Die Studie gibt Hinweise darauf, dass pädagogische Fach- und Lehrkräfte, die bereits viele Fortbildungen besucht haben, sowohl ihr Wissen als auch ihre Motivation, Kinder beim entdeckenden und forschenden Lernen zu begleiten, höher einschätzen als jene, die an nur wenigen Fortbildungen teilgenommen haben. ${ }^{20}$ Weiterhin deuten die Vergleiche darauf hin, dass Befragte mit MINT-Hintergrund ${ }^{21}$ und längerer Berufserfahrung ${ }^{22}$ motivierter für das Entdecken und Forschen sind als Befragte ohne MINT-Hintergrund bzw. mit weniger Berufserfahrung.

Als besonders wichtige Rahmenbedingungen für den Erfolg der Fortbildungen für die MINT-bezogene Professionalisierung wurden in der Studie das eigene Forschen und Entdecken, die Verknüpfung von Theorie und Praxis in der Fortbildung sowie das regelmäßige und vielfältige Fortbildungsangebot der Stiftung benannt.

In der stiftungsinternen Längsschnittbefragung wurde untersucht, welche durchschnittliche kurz- und mittelfristige Kompetenzentwicklung Pädagoginnen und Pädagogen nach dem Besuch einer „Haus der kleinen Forscher“-Präsenzfortbildung zeigen, wie diese mit individuellen Eigenschaften sowie organisationalen Rahmenbedingungen zusammenhängt und inwieweit das Vorwissen (Kompetenzausgangsniveau) der Pädagoginnen und Pädagogen von der Anzahl bereits besuchter Fortbildungen und der Fortbildungsfrequenz (Anzahl bereits besuchter Fortbildungen pro Jahr) abhängt.

Die Ergebnisse zeigen für die befragten pädagogischen Fach- und Lehrkräfte eine kurz- und mittelfristige Kompetenzentwicklung durch den Fortbildungsbe-

20 Gruppenvergleich mit den vier Gruppen 1 bis 2, 3 bis 6, 7 bis 9 und mehr als 9 besuchte Fortbildungen.

21 Von einem MINT-Hintergrund wurde gesprochen, wenn ein MINT-Fach Teil der Ausbildung der Befragten war oder diese unabhängig von den Stiftungsangeboten bereits eine Weiterbildung im MINTBereich besucht hatten.

22 In Jahren; Gruppenvergleich mit den drei Gruppen Berufsanfängerinnen/-anfänger (0 bis 2 Jahre), Fortgeschrittene (3 bis 10 Jahre) und Profis (mehr als 10 Jahre). 
such bei der Initiative „Haus der kleinen Forscher“. Dabei scheinen Personen, die ihre Kompetenzen vor der Fortbildung vergleichsweise gering einschätzen, von dem Besuch einer Fortbildung besonders zu profitieren. Personen mit weniger Berufserfahrungen scheinen vor allem kurzfristig stärker von dem Besuch einer Fortbildung zu profitieren. Für die organisationalen Rahmenbedingungen der Einrichtung wird ein Zusammenhang zwischen dem MINT-bezogenen Betreuungsschlüssel $^{23}$ und der Kompetenzentwicklung ersichtlich. Des Weiteren schätzen Pädagoginnen und Pädagogen, die bereits viele Fortbildungen besucht haben, ihr Kompetenzausgangsniveau selbst höher ein. Die Fortbildungsfrequenz (Anzahl zuvor besuchter „Haus der kleinen Forscher“-Fortbildungen pro Jahr) zeigt einen Zusammenhang mit den Vorkenntnissen im fachdidaktischen Wissen.

Die Ergebnisse der „EpFL MINT“-Studie sowie der stiftungsinternen Längsschnittbefragung weisen in eine ähnliche Richtung. Beide Untersuchungen weisen auf einen Zusammenhang zwischen der Anzahl besuchter „Haus der kleinen Forscher“-Präsenzfortbildungen und der Kompetenzentwicklung der teilnehmenden Pädagoginnen und Pädagogen hin. Dieser deutet sich in beiden Untersuchungen in Hinblick auf die Entwicklung des fachdidaktischen Wissens und der Motivation an. Des Weiteren legen beide Untersuchungen nahe, dass weniger erfahrene Personen stärker vom Besuch einer „Haus der kleinen Forscher“-Fortbildung profitieren als Personen mit mehr Erfahrung.

Durch die unterschiedliche methodische Herangehensweise konnten die Ergebnisse der explorativen „EpFL MINT“-Studie durch die Erkenntnisse der stiftungsinternen Längsschnittbefragung vertieft und ergänzt werden. Die Ergebnisse der beiden Untersuchungen geben Hinweise und Einblicke in den Professionalisierungsprozess von Pädagoginnen und Pädagogen, anhand derer die Stiftung ihre Bildungsangebote wirkungs- und bedarfsorientiert (weiter-)entwickelt. Mit Blick auf die biografische professionelle Entwicklung von Pädagoginnen und Pädagogen in der frühen MINT-Bildung knüpft die Stiftung an die bisherigen kompetenzorientierten Wirkungsstudien an (Stiftung Haus der kleinen Forscher, 2018b) und gewinnt weitere Erkenntnisse zur Wirkung ihrer Fortbildungsangebote sowie zu den Lernbedarfen der teilnehmenden pädagogischen Fach- und Lehrkräfte. Hierdurch kann die Stiftung Pädagoginnen und Pädagogen noch bessere Orientierung über das bestehende Bildungsangebot geben, und pädagogische Fach-, Lehr- und Leitungskräfte können selbst das am besten passende Thema oder Format wählen, welches sie für ihre weitere professionelle Entwicklung nutzen wollen. Dies

23 Der MINT-bezogene Betreuungsschlüssel stellt das Verhältnis zwischen der Anzahl derjenigen Pädagoginnen und Pädagogen, die zu MINT-Bildungsinhalten entdecken und forschen, und der Anzahl der Kinder in der Einrichtung dar. 
entspricht dem Menschenbild eigenaktiver Lernender, das dem pädagogischen Ansatz der Stiftung zugrunde liegt (Stiftung Haus der kleinen Forscher, 2019b).

Die Empfehlungen und Erkenntnisse aus beiden Untersuchungen nutzt die Stiftung für eine systematische Reflexion und eine bedarfs- und wirkungsorientierte (Weiter-)Entwicklung ihrer Bildungsangebote (s. u.). Im Folgenden werden zunächst die Limitationen der „EpFL MINT“-Studie sowie der stiftungsinternen Längsschnittbefragung berücksichtigt, um die Ergebnisse für die Angebotsentwicklung der Stiftung einordnen zu können. 


\section{Grenzen der Untersuchungsergebnisse}

Für die Interpretation der Ergebnisse der explorativen „EpFL MINT“-Studie ist insbesondere zu berücksichtigen, dass es sich dabei um ein Querschnittdesign handelt, anhand dessen nur eingeschränkt Aussagen über unterschiedliche Phasen in Entwicklungsprozessen getroffen werden können. Ein Längsschnittdesign war aufgrund der Rahmenbedingungen und der vorgegebenen Studienlaufzeit nicht umsetzbar. Zusätzlich ist zu berücksichtigen, dass die verschiedenen Gruppen (erfahrene und weniger erfahrene pädagogische Fach- und Lehrkräfte) nicht unter Kontrolle der anderen Hintergrundvariablen miteinander verglichen wurden. Im Fokus der Studie stand, erste Hinweise und Einblicke in die professionelle Entwicklung pädagogischer Fach- und Lehrkräfte in der frühen MINT-Bildung zu erhalten, die über die explorative Herangehensweise und das Mixed-Methods-Design der Studie gewonnen wurden.

In Hinblick auf die Stichprobe der „EpFL MINT“-Studie ist festzuhalten, dass 30 Prozent der Einrichtungen, in denen die befragten pädagogischen Fach- und Lehrkräfte tätig sind, zum Zeitpunkt der Befragung bereits als „Haus der kleinen Forscher“ zertifiziert waren. Damit weist die befragte Stichprobe einen höheren Anteil an zertifizierten Einrichtungen auf als die Gesamtheit der an den Stiftungsangeboten teilnehmenden Einrichtungen. Dies könnte darauf hinweisen, dass vorrangig besonders motivierte und an der frühen MINT-Bildung interessierte pädagogische Fach- und Lehrkräfte an der Befragung teilgenommen haben. Dafür spricht auch die eher geringe Rücklaufquote in der Online-Befragung dieser Studie, für die ein sehr umfangreicher Fragebogen ausgefüllt werden musste.

Sowohl für die externe „EpFL MINT“-Studie als auch die stiftungsinterne Längsschnittbefragung ist zu berücksichtigen, dass die MINT-bezogenen Kompetenzen anhand von Selbstberichten der Pädagoginnen und Pädagogen erhoben wurden. Dabei kann es zu einer Über- oder Unterschätzung der eigenen Kompetenzen kommen (Bortz \& Döring, 2006; Kunter \& Klusmann, 2010). Es kann angenommen werden, dass die Verzerrung der Selbsteinschätzung im Verlauf der professionellen Entwicklung variiert (Brovelli, Bölsterli, Rehm \& Wilhelm, 2013). Da die beiden Untersuchungen den Professionalisierungsprozess zu verschiedenen Zeitpunkten in den Blick nehmen, kann nicht eindeutig von einer Über- oder Unterschätzung ausgegangen werden.

Um einschätzen zu können, ob es im Verlauf der Längsschnittbefragung zu einer systematischen Verzerrung der Stichprobe gekommen ist, wurde eine Dropout-Analyse durchgeführt. Diese weist darauf hin, dass die zu drei Messzeitpunkten befragten Pädagoginnen und Pädagogen im Vergleich zu den pädagogischen Fach-, Lehr- und Leitungskräften, die nach der Ersterhebung nicht weiter an der 
Befragung teilnahmen, leicht motivierter sind und sich geringfügig selbstwirksamer einschätzt, mit Kindern zu entdecken und zu forschen.

Für die Einordnung der Ergebnisse der stiftungsinternen Längsschnittbefragung ist auch die Güte der Regressionsmodelle zu berücksichtigen. Für die kurz- und mittelfristige Kompetenzentwicklung ist die Güte der Regressionsmodelle zufriedenstellend. Die Güte der Modelle zu Zusammenhängen mit dem Kompetenzausgangsniveau der Pädagoginnen und Pädagogen ist dagegen eher gering. Es lässt sich vermuten, dass bedeutende individuelle Eigenschaften und organisationale Rahmenbedingungen noch nicht erfasst wurden. Beispielsweise könnten Variablen zum Teamklima ebenfalls mit der Kompetenzentwicklung der pädagogischen Fach-, Lehr- und Leitungskräfte im Zusammenhang stehen. Dazu bedarf es weiterer Untersuchungen (siehe Ausblick, in diesem Band). Zudem werden in künftigen Erhebungen höhere Fallzahlen angestrebt, indem die Stichprobe einerseits um neue Teilnehmende ergänzt wird und der Fragebogenrücklauf andererseits durch den parallelen Einsatz verschiedener Erhebungsmethoden erhöht werden soll.

Die Ergebnisse der Längsschnittbefragung weisen darauf hin, dass Pädagoginnen und Pädagogen, die ihre Kompetenzen bereits vor dem Besuch der betrachteten Fortbildung als sehr hoch einschätzten, diese nach der Fortbildung nicht signifikant höher einschätzen. Es ist nicht auszuschließen, dass die verwendeten Befragungsinstrumente die professionelle Entwicklung ab einem bestimmten Kompetenzniveau nicht mehr gut abbilden können. Möglicherweise waren die verwendeten Items zu leicht, um eine Entwicklung von Personen mit einem hohen Ausgangsniveau erfassen zu können. Dies wird bei weiteren Untersuchungen im Rahmen der Längsschnittbefragung berücksichtigt und noch einmal genauer in den Blick genommen.

Weder die „EpFL MINT“-Studie noch die stiftungsinterne Längsschnittbefragung ermöglichen aufgrund des gewählten Studiendesigns eindeutige Aussagen über kausale Effekte. Durch das Prä-Post-Design der Längsschnittbefragung können dennoch bedeutsame Effekte des Fortbildungsbesuchs unter Berücksichtigung von Kontrollvariablen identifiziert werden.

Insgesamt bleibt zu berücksichtigen, dass die Ergebnisse beider Untersuchungen für eine etwas motiviertere Gruppe im Vergleich zu der Gesamtheit der Nutzerinnen und Nutzer der Fortbildungen des „Hauses der kleinen Forscher“ gelten. Weitere Untersuchungen sind nötig, um genauere Erkenntnisse über alle Nutzenden zu gewinnen, auch jene, die nicht besonders hoch motiviert sind. 


\section{Umgang mit den Empfehlungen und Erkenntnissen aus den Untersuchungen}

Aus den Ergebnissen der externen „EpFL MINT“-Studie hat die Forschungsgruppe Empfehlungen zur (Weiter-)Entwicklung der Stiftungsangebote abgeleitet (siehe Kapitel A 11).

Die Forschungsgruppe berichtet, dass die befragten pädagogischen Fachund Lehrkräfte die Fortbildungen der Initiative „Haus der kleinen Forscher“ sehr wertschätzen und positiv bewerten und als prägendes Ereignis in ihrem Professionalisierungsprozess sehen. Als besonders positiv heben die Befragten die leichte Umsetzbarkeit der Praxisbeispiele und die Praxisanregungen für die pädagogische Arbeit in der eigenen Einrichtung sowie die Möglichkeiten zum Austausch mit anderen Pädagoginnen und Pädagogen hervor.

In Hinblick auf Verbesserungsvorschläge wünschen sich die in der „EpFL MINT“-Studie befragten Pädagoginnen und Pädagogen mehr konkrete Tipps zum Forschen mit den Kindern und eine stärkere Ausrichtung an der Alltagswelt der Kinder. Ebenso scheint es einen Bedarf für eine stärkere Berücksichtigung der unterschiedlichen Altersgruppen der Kinder, insbesondere der Grundschulkinder, zu geben. Als weiteren Aspekt nennen die befragten Pädagoginnen und Pädagogen die stärkere Fokussierung auf didaktische Aspekte sowie das pädagogische Selbstverständnis als Fach- beziehungsweise Lehrkraft. Sie äußern zudem den Wunsch, häufiger an den „Haus der kleinen Forscher“-Fortbildungen teilnehmen zu können.

Darauf aufbauend empfiehlt die Forschungsgruppe der Stiftung weiterhin darauf zu achten, dass Fortbildungsinhalte gut und mit bereits vorhandenen Materialien in der pädagogischen Praxis mit den Kindern umgesetzt werden können. Dabei sollten ebenso der theoretische Input und Praxiserfahrungen gezielter

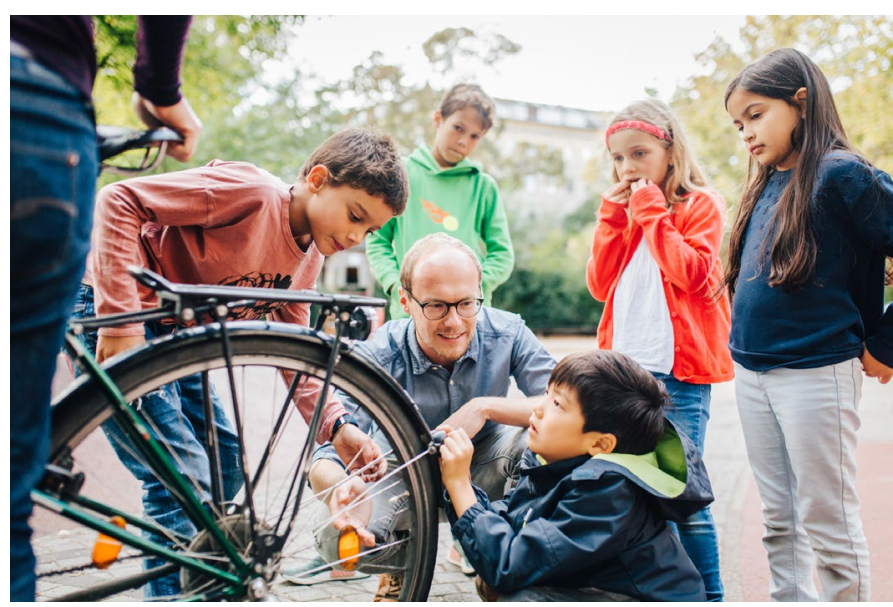
verzahnt sowie auf die Bedeutung des Fachwissens hingewiesen werden. Zur stärkeren Ausrichtung an Grundschulkindern könnten weitere Themen ergänzt und die Inhalte bisheriger Fortbildungen für unterschiedliche Altersgruppen aufbereitet werden. Die Forschungsgruppe empfiehlt außerdem, den Bezug zwischen MINT-spezifischen Fragestellungen und der Alltagswelt der Kin- 
der stärker herauszustellen. Da die Pädagoginnen und Pädagogen insbesondere den persönlichen Austausch als positiv erleben, befürwortet die Forschungsgruppe, in den Fortbildungen weiterhin genügend Zeit und Raum dafür zur Verfügung zu stellen. Als ein zentrales Unterstützungselement für pädagogische Fach- und Lehrkräfte beim Forschen mit Kindern wurde in der „EpFL MINT“-Studie der „Forschungskreis“ (vgl. Marquardt-Mau, 2004, 2011; Rank et al., 2018; Stiftung Haus der kleinen Forscher, 2013) besonders in den Blick genommen. Das didaktische Konzept einer naturwissenschaftlichen Grundbildung zum Forschen mit Kindern und das damit verbundene Modell des Forschungskreislaufs wurde von Frau Marquardt-Mau entwickelt (2004) und im pädagogischen Ansatz der Stiftung „Haus der kleinen Forscher“ adaptiert. ${ }^{24}$ Zwar fällt es den befragten Pädagoginnen und Pädagogen nicht leicht, diesen umzusetzen. Sie beschreiben ihn aber als gute Orientierungshilfe und Handlungsempfehlung. Die Forschungsgruppe empfiehlt daher, die Methode „Forschungskreis“ (vgl. Marquardt-Mau, 2004, 2011; Rank et al., 2018; Stiftung Haus der kleinen Forscher, 2013) weiterhin in die Fortbildungen zu integrieren.

Insgesamt leitet die Forschungsgruppe aus den Ergebnissen der Studie und den Wünschen der pädagogischen Fach- und Lehrkräfte Schlussfolgerungen für die (Weiter-)Entwicklung der Stiftungsangebote ab. Sie empfiehlt der Stiftung „Haus der kleinen Forscher“, möglichst vielen Pädagoginnen und Pädagogen zu ermöglichen, häufig an den Angeboten der Stiftung teilzunehmen. Dies könnte durch eine Ausweitung der Online-Formate, einen höheren Angebotsturnus und eine Erweiterung der regionalen Verteilung der Präsenzformate umgesetzt werden. Dafür empfiehlt die Forschungsgruppe weitere Untersuchungen, um Angebot und Nutzung der Fortbildungsformate gut abzustimmen.

Auch aus den Erkenntnissen der stiftungsinternen Längsschnittbefragung lassen sich Schlussfolgerungen für die weitere wirkungs- und bedarfsorientierte Angebotsentwicklung ableiten. Die Ergebnisse der stiftungsinternen Längsschnittbefragung weisen in eine ähnliche Richtung wie die „EpFL MINT“-Studie und betonen den Mehrwert der Anzahl besuchter „Haus der kleinen Forscher“Präsenzfortbildungen für die Kompetenzentwicklung der Pädagoginnen und Pädagogen hin, insbesondere in Hinblick auf die Entwicklung des fachdidaktischen Wissens und der Motivation sowie für weniger erfahrene Personen. Als zentrale Schlussfolgerung deutet sich aus der Längsschnitterhebung zudem an, dass auch organisationale Rahmenbedingungen in der Einrichtung für die Verstetigung von Kompetenzen bedeutsam sind (siehe Kapitel B 4). Die Unterstützung der gesamten Bildungseinrichtung könnte damit ebenfalls förderlich sein für die Umsetzung der frühen MINT-Bildung in der pädagogischen Praxis. 
Die Empfehlungen aus der externen „EpFL-MINT“-Studie sowie die Erkenntnisse aus der stiftungsinternen Längsschnittbefragung lassen sich zu folgenden vier zentralen Punkten zusammenfassen:

- Angebot eines kontinuierlichen Fortbildungsprogramms mit Austauschphasen

- Verzahnung von Theorie und Praxis

- Differenzierung nach Altersgruppen, insbesondere für Kita- und Grundschulkinder

- Unterstützung der gesamten Bildungseinrichtung

Im Folgenden werden die Maßnahmen der Stiftung zum Umgang mit den Empfehlungen und den Erkenntnissen aus beiden Untersuchungen beschrieben. Abschließend wird ein Ausblick auf zukünftige Fragestellungen und Erkenntnisbedarfe gegeben, mit denen sich die Stiftung im Rahmen ihrer fachlichen Fundierung und wissenschaftlichen Begleitforschung beschäftigt, um ihre Angebote bedarfs- und wirkungsorientiert (weiter-)zu entwickeln.

\section{Angebot eines kontinuierlichen Fortbildungsprogramms mit Austauschphasen}

Die „EpFL MINT“-Studie zeigt einen positiven Zusammenhang zwischen der Entwicklung der MINT-bezogenen professionellen Kompetenzen von pädagogischen Fach- und Lehrkräften und der Anzahl der besuchten Fortbildungen. Dies lässt vermuten, dass eine wiederholte und damit kontinuierliche Fortbildungsteilnahme die Entwicklung der professionellen MINT-bezogenen Kompetenzen von pädagogischen Fach- und Lehrkräften unterstützt. Zusätzlich berichtet die „EpFL MINT“Studie, dass sich die befragten Pädagoginnen und Pädagogen einen hohen Angebotsturnus und eine große Themenvielfalt wünschen, um möglichst mehrere „Haus der kleinen Forscher“-Fortbildungen im Jahr besuchen zu können (siehe Kapitel A 11).

Ergänzend dazu ermöglichen die Ergebnisse der stiftungsinternen Längsschnittbefragung ein differenziertes Bild zum Zusammenhang der Fortbildungsanzahl und -frequenz mit dem Kompetenzniveau der teilnehmenden Pädagoginnen und Pädagogen. Es zeigt sich, dass die Fortbildungsanzahl in Zusammenhang mit dem selbsteingeschätzten Ausgangsniveau des fachdidaktischen Wissens, der Motivation und der Selbstwirksamkeit, mit Kindern zu entdecken und zu forschen, steht. Die Fortbildungsfrequenz weist einen Zusammenhang mit dem fachdidaktischen Wissen auf (siehe Abschnitt B 4.2). Um diese Zusammenhänge der Fortbildungsanzahl und -frequenz mit der Kompetenzentwicklung zu nutzen, erscheint ein aufeinander aufbauendes Fortbildungsformat mit aufeinander be- 
zogenen Modulen, Phasen für die Praxiserprobung sowie Gelegenheiten für Austausch und Reflexion als besonders förderlich.

Entsprechende Bedarfe wurden auch in der „EpFL MINT“-Studie von den pädagogischen Fach- und Lehrkräften benannt. Insbesondere den pädagogischen Fachkräften ist der kollegiale Austausch zum pädagogischen Alltag in den Einrichtungen wichtig (siehe Kapitel A 11). Die Forschungsgruppe der „EpFL MINT“Studie weist diesbezüglich auf die Möglichkeiten von Online-Formaten hin. Eng verzahnt mit den Präsenzfortbildungen könnten diese sowohl als Austauschplattform als auch zum Selbststudium über die Präsenzfortbildungen hinaus genutzt werden. Die höhere Flexibilität könnte auch zu einer Erhöhung der Fortbildungsanzahl sowie der Fortbildungsfrequenz führen.

Zum Zeitpunkt der Befragung wurden die digitalen Angebote der Stiftung weniger von den pädagogischen Fach- und Lehrkräften genutzt. Eine mögliche Ursache dafür könnte die technische Ausstattung am Arbeitsplatz der Pädagoginnen und Pädagogen darstellen. Des Weiteren könnte die geringe Nutzung der Online-Angebote durch Zeitmangel begründet sein. Weitere Untersuchungen sind notwendig, um empirisch fundierte Erkenntnisse über die Bedeutung verschiedener Einflussfaktoren auf die Nutzung der digitalen Angebote gewinnen zu können.

Die Ergebnisse der stiftungsinternen Längsschnittbefragung weisen zusätzlich darauf hin, dass aufeinander aufbauende Fortbildungen für unterschiedliche Kompetenzniveaus die Kompetenzentwicklung von Pädagoginnen und Pädagogen besonders fördern könnte.

\section{Umsetzung in den Angeboten der Stiftung}

In mehreren Projekten im Kita- und Grundschulbereich sammelt die Stiftung bereits Erfahrungen mit Fortbildungsprogrammen, die sich durch abwechselnde Fortbildungs- und Praxisphasen auszeichnen und eine enge Begleitung durch den Einsatz digitaler Lernformate, hohe Flexibilität sowie einen dauerhaften Austausch zwischen den teilnehmenden pädagogischen Fach-, Lehr- und Leitungskräften ermöglichen. Es wurden bereits erste Fortbildungsformate umgesetzt und in der Fläche zur Verfügung gestellt, die aus aufeinander aufbauenden Fortbildungen bestehen. Außerdem wurden Fortbildungsreihen entwickelt, die als Blended-Learning-Formate Online- mit Präsenzangeboten verbinden. Insgesamt hat die Stiftung ihre digitalen Angebote erweitert und bietet den pädagogischen Fach- und Lehrkräften themenspezifische Foren auf einer digitalen Plattform, um sich über Fortbildungsinhalte auszutauschen.

Neben den eintägigen flexibel wählbaren Präsenzfortbildungen bietet die Stiftung seit 2018 zwei aufeinander aufbauende Fortbildungen zum Bildungskonzept Bildung für nachhaltige Entwicklung (BNE) an (2016-2022, gefördert durch das Bundesministerium für Bildung und Forschung). Die Fortbildung „Tür auf! 
Mein Einstieg in Bildung für nachhaltige Entwicklung“ bietet einen breiten und offenen Zugang zu BNE. In der darauf aufbauenden Fortbildung „Macht mit! Bildung für nachhaltige Entwicklung in der Praxis“ werden die Praxiserfahrungen der Teilnehmenden ins Zentrum gestellt. Die Teilnehmenden reflektieren ihre eigene Praxis und entwickeln diese weiter (Stiftung Haus der kleinen Forscher, 2019a).

In zwei weiteren Projekten sammelt die Stiftung aktuell Erfahrungen mit Fortbildungsprogrammen, bei denen die enge Begleitung der pädagogischen Fachund Lehrkräfte im Fokus steht und dafür die Möglichkeiten des Online-Lernens genutzt werden.

Das Projekt „KiQ - gemeinsam für Kita-Qualität: Wenn Entdecken und Forschen zum Alltag werden“ (2019-2022, gefördert vom Bundesministerium für Bildung und Forschung) ${ }^{25}$ soll pädagogische Fach- und Leitungskräfte in Kitas dabei unterstützen, das entdeckende und forschende Lernen als gemeinsames pädagogisches Selbstverständnis in ihrem Alltag langfristig zu etablieren und damit die pädagogische Qualität in ihrer Einrichtung zu steigern. Die Stiftung entwickelt und erprobt dazu zunächst in einem Modellprojekt vielfältige Unterstützungsmaßnahmen zur Verankerung des entdeckenden und forschenden Lernens in Kitas.

Damit das entdeckende und forschende Lernen gelebter Alltag in den teilnehmenden Kitas wird, kombiniert das „KiQ“-Modellprogramm mehrere Bausteine:

- eine Fortbildungsreihe, an der für jede Einrichtung ein Tandem aus einer pädagogischen Fachkraft und der Kita-Leitung teilnimmt,

- zwei Inhouse-Fortbildungen für das gesamte Kita-Team zur aktiven Einbindung des Kollegiums,

- eine Prozessbegleitung, die jede teilnehmende Einrichtung individuell bei ihrem Entwicklungsprozess unterstützt, und

- viel Raum für kollegialen Erfahrungsaustausch zwischen den teilnehmenden Kitas - in der Region und über die digitale Lernplattform der Stiftung „Haus der kleinen Forscher“.

Den zentralen Pfeiler des Kita-Programms bildet eine Fortbildungsreihe aus fünf aufeinander aufbauenden Modulen. Sie vermittelt umfangreiche Fach- und Methodenkenntnisse rund ums entdeckende und forschende Lernen im Kita-Alltag und die für dessen Verankerung förderlichen Ansätze aus der Organisationsentwicklung. Die Fortbildungsreihe erstreckt sich über 18 Monate und beinhaltet sowohl Präsenz- als auch Online-Angebote (Blended Learning). In der Zeit zwischen

25 Laufzeit Gesamtprojekt: 1. März 2019 bis 31. Juli 2022, weitere Informationen zum Projekt sind unter hdkf.de/kiq-modellphase verfügbar. 
den Modulen wird auf vielfältige Weise der Transfer in die Praxis unterstützt (siehe Abbildung 21).

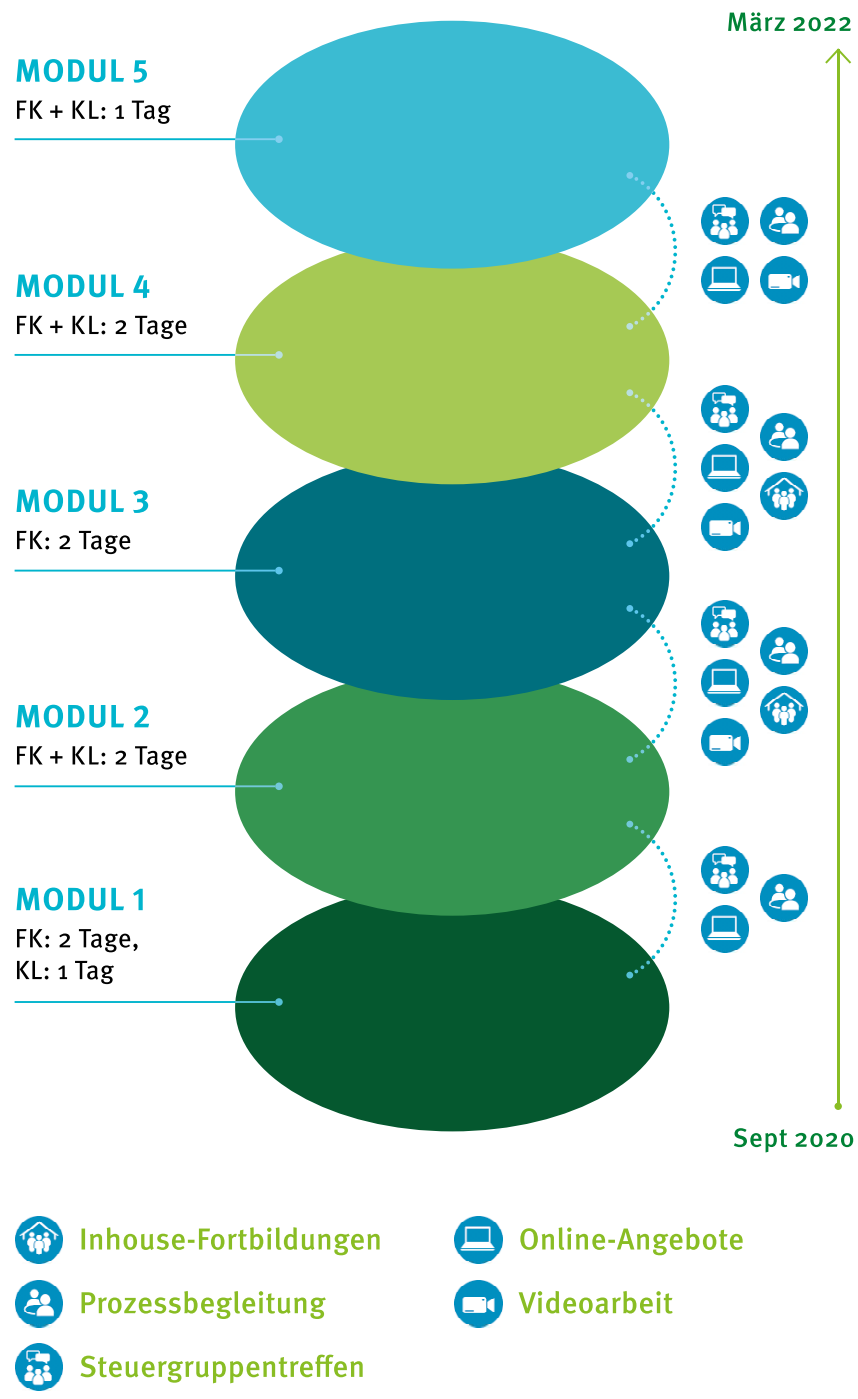

Abbildung 21. Aufbau des „KiQ“-Modellprogramms

Im Projekt „Prima!Start“(2019-2020, gefördert durch das Bundesministerium für Bildung und Forschung), das sich an Lehrkräfte im Grundschulunterricht richtet, wurde erstmals ein Blended-Learning-Format entwickelt. Dieses besteht aus sich abwechselnden und aufeinander bezugnehmenden Online- und Präsenzphasen. In einer ausgeprägten Praxisphase können die Lehrkräfte ihre Ideen erproben, reflektieren und überarbeiten. Durch den gezielten Einsatz von Online-Angeboten 
als fester Bestandteil des Fortbildungskonzepts findet die Erprobung nicht isoliert an der einzelnen Schule statt, sondern gemeinsam mit allen teilnehmenden Lehrkräften und den Trainerinnen oder Trainern. Die Lehrkräfte werden angeregt, ihren Unterricht zu reflektieren und sich weiter hinsichtlich des entdeckenden und forschenden Lernens im MINT-Kontext zu professionalisieren. Die Fortbildung ist auf eine Dauer von zwölf Wochen angelegt, sodass genug Zeit ist, Praxis- und Reflexionsphasen miteinander zu verbinden und damit eine Haltungsarbeit zu ermöglichen.

Nach Abschluss der Fortbildung steht es den Lehrkräften frei, weiter im Kontakt zu bleiben und über die Online-Lernplattform gemeinsam weitere Ideen zu entwickeln (siehe Abbildung 22). Dazu steht ein geschlossenes Forum zur Verfügung, das nur die Teilnehmenden der Fortbildung sehen können. Sie können sich außerdem gegenseitig Nachrichten schreiben sowie Materialien in den gemeinsam genutzten Online-Materialpool der Fortbildung hochladen. Langfristig ist geplant, die Möglichkeiten der Plattform noch stärker zu nutzen, die an ein soziales Netzwerk erinnern. Nutzerinnen und Nutzer könnten dann beispielsweise auf der Startseite sehen, was Kolleginnen und Kollegen aus der eigenen Fortbildung zuletzt auf der Plattform unternommen haben oder welche Präsenz-Veranstaltungen im eigenen oder in angrenzenden Netzwerken als nächstes geplant sind. Zudem soll perspektivisch die Online-Plattform für alle pädagogischen Fach-, Lehr- und Leitungskräfte zur Verfügung stehen und ein Empfehlungsalgorithmus den Nutzerinnen und Nutzern nach Abschluss einer Fortbildung Vorschläge machen, welche Kurse sich als nächstes anbieten. Dies wäre eine zukünftige Möglichkeit, allen Teilnehmenden mit ihren unterschiedlichen Kompetenzniveaus bedarfsorientiert zu unterstützen.

Zeitgleich mit der Entwicklung der Fortbildungsangebote wurden die Fortbildungsstrukturen der Bildungsinitiative weiterentwickelt und ausgebaut, um das Fortbildungsangebot für Lehrkräfte im unterrichtlichen Bereich der Zielgruppe zur Verfügung stellen und dieses in Kooperation mit den Bundesländern umsetzen zu können. Seit Herbst 2019 können sich Lehrkräfte aus Hessen fortbilden. Diese Fortbildungen werden im Rahmen des Projekts „Prima!Hessen“ vom Kultusministerium Hessen finanziert. Weitere Länder sollen folgen. 


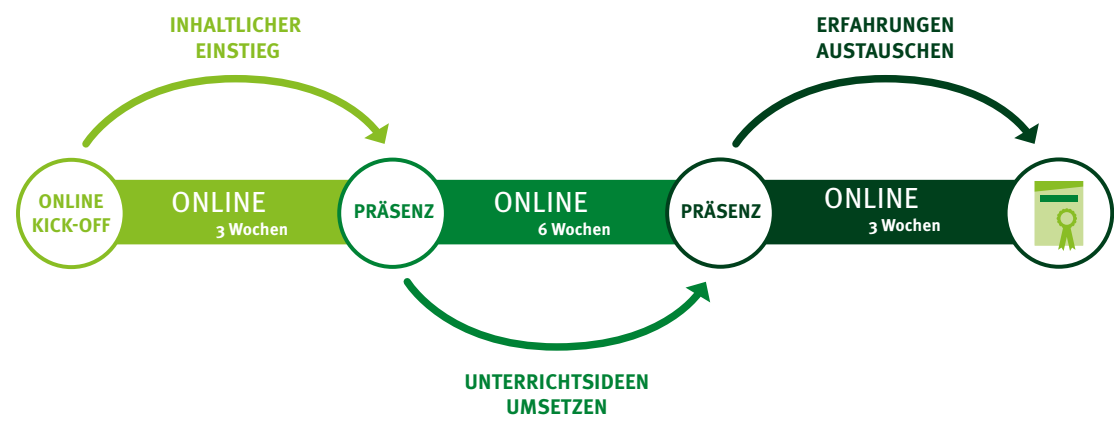

Abbildung 22. Fortbildungsablauf am Beispiel „Informatische Bildung im Grundschulunterricht“"

\section{Verzahnung von Theorie und Praxis}

Die Stiftung „Haus der kleinen Forscher“ legt bei ihrer Angebotsentwicklung großen Wert auf die enge Verzahnung von theoretischem Hintergrundwissen und alltagsnahen Praxisanregungen. Sie verfolgt damit einen praxisnahen Ansatz, der es den Pädagoginnen und Pädagogen ermöglicht, ihre Kenntnisse und Kompetenzen zu erweitern und in der alltäglichen Arbeit mit den Kindern einzusetzen.

Bestätigend zeigt sich in der externen Studie „EpFL MINT“, dass die pädagogischen Fach- und Lehrkräfte die Verknüpfung von Theorie und Praxis besonders häufig als förderliches Merkmal der „Haus der kleinen Forscher“-Fortbildungen für ihre Professionalisierung benennen (siehe Kapitel A 11). Gleichzeitig weist die Studie darauf hin, dass sich die befragten pädagogischen Fach- und Lehrkräfte mehr konkrete Tipps zum Entdecken und Forschen mit Kindern wünschen, dass die Umsetzbarkeit in der Praxis noch stärker berücksichtigt wird und sich die Inhalte noch enger am Alltag der Kinder orientieren. Die Forschungsgruppe empfiehlt in diesem Zusammenhang den theoretischen Input eng mit den Praxiserfahrungen zu verzahnen. Des Weiteren wünschen sich die Befragten der „EpFL MINT“-Studie eine Fokussierung auf didaktische Aspekte sowie das pädagogische Selbstverständnis als Fach- beziehungsweise Lehrkraft.

Die Ergebnisse der Längsschnittbefragung zeigen, dass die Pädagoginnen und Pädagogen nach dem Besuch einer „Haus der kleinen Forscher“-Präsenzfortbildung ihr fachdidaktisches Wissen kurz- und mittelfristig im Durchschnitt höher einschätzen, sich als leicht motivierter empfinden, mit Kindern zu entdecken und zu forschen, und sich dies auch eher zutrauen. Es deutet sich dabei ein stärkerer Zusammenhang des Fortbildungsbesuchs mit dem fachdidaktischen Wissen an als mit den Kompetenzen Motivation und Selbstwirksamkeit. Letztere wurden von den Befragten bereits vor dem Fortbildungsbesuch als sehr hoch eingeschätzt. Im Rahmen der Interviews, die bei der „EpFL MINT“-Studie durchgeführt wurden, 
betonten die pädagogischen Fach- und Lehrkräfte, dass das eigenständige Entdecken und Forschen in den Fortbildungen sie darin unterstützt, Hemmnisse hinsichtlich des Forschens im MINT-Bereich abzubauen. Selbst ins Handeln zu kommen und aktiv zu entdecken und zu forschen, scheint erwartungsgemäß förderlich dafür zu sein, dass die pädagogischen Fach- und Lehrkräfte es sich zutrauen, auch mit Kindern zu entdecken und zu forschen.

Beide Untersuchungen bestätigen, dass der Fokus auf eine noch engere Verzahnung von Theorie und Praxis sowie eine stärkere Unterstützung der Pädagoginnen und Pädagogen in ihrer Rolle als Lernbegleitungen durch Praxiserfahrungen und eine starke Orientierung an der Alltagswelt der Kinder sowie die Umsetzung des entdeckenden und forschenden Lernens im pädagogischen Alltag der Einrichtungen noch besser fördern könnten.

\section{Umsetzung in den Angeboten der Stiftung}

Die Stiftung verfolgt mit ihrem Fortbildungsangebot insgesamt eine alltagsintegrierte MINT-Bildung für nachhaltige Entwicklung, die sich dadurch auszeichnet, dass sie sich am Kind orientiert und in fast allen Situationen des pädagogischen Alltags stattfinden kann (Stiftung Haus der kleinen Forscher, 2019c). Die Erkenntnisse der Studie bestätigen die Bedeutung des pädagogischen Ansatzes der Stiftung (Stiftung Haus der kleinen Forscher, 2019d).

Mit dem Grundlagenseminar zum pädagogischen Ansatz bietet die Stiftung eine Fortbildung zum pädagogischen Konzept des „Hauses der kleinen Forscher“, das die theoretische und methodische Grundlage sämtlicher Bildungsangebote der Stiftung bildet. In dem Grundlagenseminar erfahren die Pädagoginnen und Pädagogen unter anderem die Entwicklungsgrundlagen des Lernens und setzen sich mit ihrem Bild vom Kind auseinander. Sie vertiefen ihr Wissen über die kokonstruktive Begleitung der Kinder beim „Denkenlernen“ in gemeinsamen Entdeckungs- und Forschungssituationen. Sie erhalten grundlegende Kenntnisse der MINT-spezifischen fachdidaktischen Fragestellungen und reflektieren Gemeinsamkeiten und Unterschiede der MINT-spezifischen Fachdisziplinen. Ein Ziel des Grundlagenseminars ist zudem, pädagogische Handlungskompetenzen mit Fokus auf gute Lernbegleitung beim Entdecken und Forschen zu stärken. Diese Fortbildung ist auch für pädagogische Fach- und Lehrkräfte geeignet, die bereits mit dem pädagogischen Konzept der Stiftung in Berührung gekommen sind, da sie neue Anregungen, Möglichkeiten zur Selbstreflexion und viele Anlässe zum Austausch bietet. Sie wird zudem sowohl als Präsenzveranstaltung als auch als Online-Kurs angeboten.

Um noch stärker den Fokus auf den Alltag der Kinder zu legen und Anknüpfungspunkte in der pädagogischen Praxis zu MINT-Fragestellungen aufzuzeigen, hat die Stiftung das Bildungsangebot „MINT ist überall“ (Stiftung Haus der klei- 
nen Forscher, 2019c) entwickelt. Darin werden die Pädagoginnen und Pädagogen unterstützt, situativ Interessen der Kinder für MINT-Themen zu erkennen und aufzugreifen sowie bei ihnen Kompetenzen zu fördern, die MINT-übergreifend relevant sind. Dies greift bereits den Bedarf der pädagogischen Fach- und Lehrkräfte nach alltagsintegrierten Praxisanregungen auf und stärkt sie zugleich darin, Fragen der Kinder aufzunehmen und sie durch Impulse zum eigenen Handeln anzuregen.

Mit dem Bildungsangebot „MINT ist überall“ wird übergreifend der gesamte MINT-Bildungsbereich angesprochen. Ausgangspunkt ist der Alltag der Kinder: Wo überall finden sich hier Bezüge zu Mathematik, Informatik, Naturwissenschaften oder Technik? Wie können daraus MINT-bezogene Lernsituationen entstehen? Und wie können die Kinder dabei gut von den pädagogischen Fach- und Lehrkräften begleitet werden?

Dieses Bildungsangebot erleichtert es den Pädagoginnen und Pädagogen, das große Potenzial von Alltagssituationen für MINT-Bildung zu erkennen und eigene Ideen zu entwickeln, wie diese Gelegenheiten aufgegriffen und vertieft werden können. Es besteht aus einer Präsenzfortbildung, mehreren Online-Angeboten mit unterschiedlicher Schwerpunktsetzung sowie pädagogischen Materialien mit vielfältigen Lern- und Austauschmöglichkeiten für die pädagogischen Fach- und Lehrkräfte. Alle Formate können je nach individuellen Interessen, Bedürfnissen und zeitlichen Ressourcen unabhängig voneinander genutzt werden.

Der Austausch der Pädagoginnen und Pädagogen untereinander ist ein zentraler Aspekt des Bildungsangebots „MINT ist überall“. Dabei werden beispielsweise eigene Erfahrungen geteilt und diskutiert oder Videosequenzen von drei- bis zehnjährigen Kindern in Alltagssituationen gemeinsam betrachtet und analysiert. Die didaktischen Materialien, z. B. der Fragenfächer oder das MINT-Spiel, werden als Werkzeuge für die eigene pädagogische Arbeit kennengelernt (vgl. Materialpaket „MINT ist überall“, Stiftung Haus der kleinen Forscher, 2019b). Der handliche und flexibel einsetzbare Fragenfächer bietet Impulsfragen entlang der Gemeinsamkeiten der MINT-Disziplinen, um die Kinder beim Forschen zu begleiten. Er enthält Beispielfragen, mit denen die pädagogischen Fach- und Lehrkräfte - auch bereits in kleinen Situationen - gute MINT-Bildung fördern können. Das MINTSpiel enthält verschiedene Bildkärtchen, die einen spielerischen Einstieg in das Entdecken und Forschen im Alltag ermöglichen. Durch die vielfältigen Kombinationsmöglichkeiten ergeben die Bilder spannende Forschungsfragen. Mithilfe der Impulse auf dem Spielbrett können erfahrene Kinder auch alleine zu ihren Fragen und Ideen forschen. Das Angebot zum Fortbildungsthema „MINT ist überall“ umfasst außerdem mehrere digitale Lernangebote. Beispielsweise zur praktischen Anwendung und Reflexion der Umsetzung der pädagogischen Materialien zum Fortbildungsangebot. Insgesamt legt das Bildungsangebot den Fokus auf Metho- 
den der Lernbegleitung, die für den gesamten MINT-Bereich gelten. Seit Herbst 2019 finden die Präsenzfortbildungen für Trainerinnen und Trainer statt, sodass die Fortbildungen nun bundesweit über die Netzwerkpartner der Stiftung „Haus der kleinen Forscher“ angeboten werden und Pädagoginnen und Pädagogen daran teilnehmen können.

In Hinblick auf die inhaltliche Gestaltung der Fortbildungen der Stiftung „Haus der kleinen Forscher“ benennen die in der „EpFL MINT“-Studie befragten pädagogischen Fach- und Lehrkräfte insbesondere auch den „Forschungskreis“ (vgl. Marquardt-Mau, 2004, 2011; Rank et al., 2018; Stiftung Haus der kleinen Forscher, 2013) als gute Orientierungshilfe und Handlungsempfehlung zur Umsetzung des Forschens mit Kindern. Die Forschungsgruppe empfiehlt daher, diesen auch zukünftig in die Bildungsangebote zu integrieren und so einen stärkeren Bezug zwischen Theorie und Praxis herzustellen.

Die Stiftung „Haus der kleinen Forscher“ hat jeweils einen Mathematik-, Informatik-, Forschungs- (Naturwissenschaften) und Technikkreis entwickelt, um die pädagogischen Fach- und Lehrkräfte dabei zu unterstützen, die Kinder beim Prozess des Forschens zu begleiten - bei mathematischen, informatischen, naturwissenschaftlichen oder technischen Fragestellungen (verfügbar unter haus-derkleinen-forscher.de). Die Kreise sind als Modelle oder Werkzeuge zu verstehen, die pädagogischen Fach- und Lehrkräften zeigen, wie sie gemeinsam mit den Kindern experimentieren und in einen Dialog über Alltagsphänomene treten können. Sie beschreiben das forschende Vorgehen in jeder der MINT-Disziplinen. Alle vier Kreise sind in der Mappe „Einstiegsmaterialien der Stiftung“ enthalten, die die pädagogischen Fach- und Lehrkräfte beim ersten Besuch einer „Haus der kleine Forscher"-Fortbildung erhalten.

In der neuesten Auflage des pädagogischen Ansatzes der Stiftung „Haus der kleinen Forscher" werden ebenfalls alle vier Kreise zu Mathematik, Informatik, Naturwissenschaften und Technik aufgegriffen und als Werkzeuge zur Lernbegleitung näher erläutert (Stiftung Haus der kleinen Forscher, 2019d). Zusätzlich stellt die Stiftung verschiedene Karten-Sets zur Verfügung, die von den pädagogischen Fach- und Lehrkräften ebenfalls als unterstützende Materialien in der pädagogischen Praxis genutzt werden können. Insbesondere die Forschungskarten stellen exemplarisch dar, wie einer Frage mithilfe von einem der Kreise (z. B. Forschungskreis oder Mathematikkreis) nachgegangen werden kann. Sie zeigen, wie die Pädagoginnen und Pädagogen sich gemeinsam mit den Kindern in den Prozess des Forschens begeben und sich dabei von Vorstellungen, Vermutungen und Versuchsideen der Kinder leiten lassen können (Stiftung Haus der kleinen Forscher, 2019d). 
Differenzierung nach Altersgruppen, insbesondere für Kita- und

\section{Grundschulkinder}

Seit 2011 gehören auch Horte und Grundschulen zur Zielgruppe der Bildungsangebote der Stiftung „Haus der kleinen Forscher“. Das vielfältige Bildungsangebot kann seitdem sowohl von pädagogischen Fachkräften als auch Lehrkräften wahrgenommen werden und ist insgesamt für die Lernbegleitung von drei- bis zehnjährigen Kindern im außerunterrichtlichen Bereich konzipiert.

In der stiftungsinternen Längsschnittbefragung konnten keine Hinweise auf bedeutsame Unterschiede in der selbsteingeschätzten Kompetenzentwicklung zwischen pädagogischen Fach-, Lehr- und Leitungskräften gefunden werden. Die in der Längsschnittbefragung gezeigten Fortbildungseffekte gelten daher sowohl für pädagogische Fach- als auch Lehr- und Leitungskräfte.

Darüber hinaus hat die externe Studie „EpFL MINT“ bei den teilnehmenden pädagogischen Fach- und Lehrkräften Wünsche und Erwartungen an die „Haus der kleinen Forscher"-Fortbildungen erfragt. Hier werden zum Teil unterschiedliche Bedarfe der beiden Zielgruppen deutlich. Beispielsweise wünscht sich ein größerer Teil der Lehrkräfte andere Fortbildungsthemen im Vergleich zu den Fachkräften (siehe Abschnitt A 8.1.4). Der Wunsch nach kollegialem Austausch sowie die Beibehaltung des bisherigen Konzepts der Fortbildungen werden von den Fachkräften häufiger benannt.

Sowohl die pädagogischen Fach- als auch die Lehrkräfte wünschen sich außerdem, dass die verschiedenen Altersgruppen der Kinder stärker in den Fortbildungen berücksichtigt werden. Vermehrt wünschen sich dies zwar im Vergleich der beiden Berufsgruppen die pädagogischen Fachkräfte. Gleichzeitig werden sich insgesamt vor allem mehr Anregungen für Kinder im Grundschulalter gewünscht (siehe Kapitel A 11).

\section{Umsetzung in den Angeboten der Stiftung}

Neben den Angeboten für den Kita-Bereich hat die Stiftung „Haus der kleinen Forscher“ für die vier MINT-Disziplinen eine Sammlung an Forscherkarten-Sets speziell für die Altersgruppe der Grundschulkinder entwickelt. Dazu gehören auch ergänzende Handreichungen für Lehrkräfte zur praktischen Anwendung der Karten (verfügbar unter haus-der-kleinen-foscher.de). Die Karten-Sets greifen das Bedürfnis der Kinder auf, eigenständig zu entdecken und zu forschen und sich vor allem mit Gleichaltrigen auseinanderzusetzen. Sie beinhalten Handlungsimpulse für die Kinder, die durch zahlreiche Fotos und Symbole unterstützt werden (vgl. u. a. Stiftung Haus der kleinen Forscher, 2016). 


\section{Check dein Rad}

Bring dein Rad in Form.
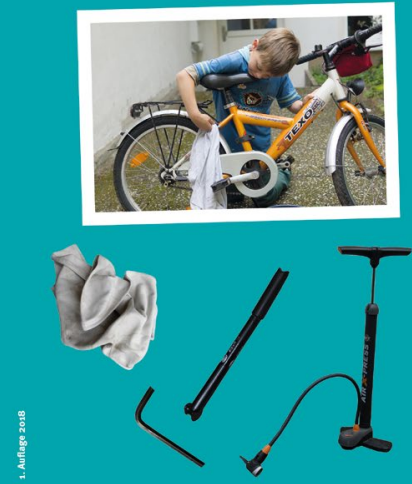

Ist alles dran und funktioniert alles?

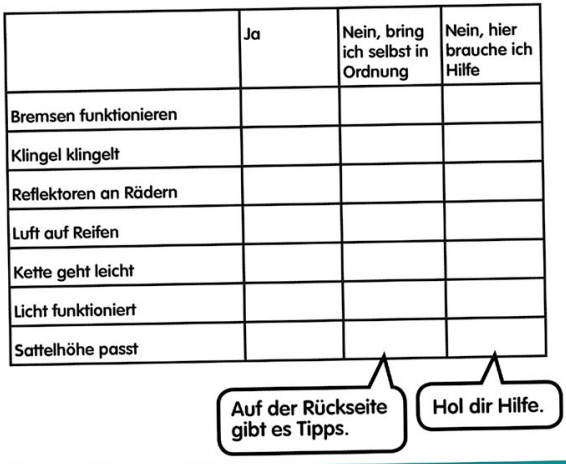

TECHNIK - VON HIER NACH DA

Check dein Rad

Hat dein Reifen genügend Luft?
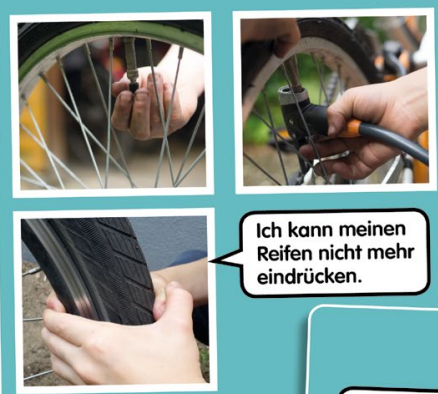

Ich kann meinen Reifen nicht mehr eindrücken.

So hat dein Sattel die richtige Höhe:

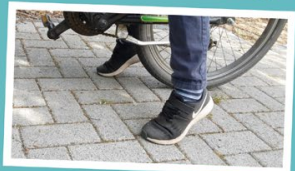

Wie verstellst du deinen Sattel?
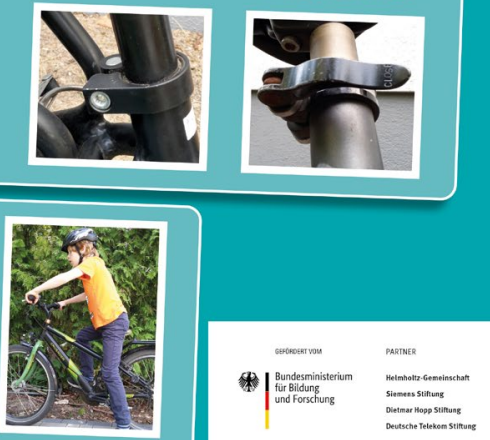

Abbildung 23. Vorder- und Rückseite der Forscherkarte „Check dein Rad" aus dem KartenSet „Technik - von hier nach da“" (Stiftung Haus der kleinen Forscher, 2018a) 
Im fünften Band der Wissenschaftlichen Schriftenreihe der Stiftung „Haus der kleinen Forscher“ (vgl. Stiftung Haus der kleinen Forscher, 2013) werden die Zieldimensionen und Qualitätskriterien einer frühen naturwissenschaftlichen Bildung erläutert, auf denen die naturwissenschaftlichen Bildungsangebote der Stiftung aufbauen. Darin werden die Zieldimensionen und deren Messung jeweils für das Kita-Alter (vgl. Anders, Hardy, Pauen \& Steffensky, 2013) sowie das Grundschul-Alter (vgl. Anders, Hardy, Sodian \& Steffensky, 2013) gesondert betrachtet und beschrieben. Zusätzlich richtet sich die darin veröffentlichte Expertise „Prozessbezogene Qualitätskriterien für den naturwissenschaftlichen Unterricht“ von Jörg Ramseger direkt an die pädagogischen Fach- und Lehrkräfte, um diese bei Unterrichtsplanung sowie der Selbstevaluation der naturwissenschaftlichen Angebote zu unterstützen (vgl. Ramseger, 2013). In den Bänden 7, 8 und 9 der Wissenschaftlichen Schriftenreihe werden ergänzend die Zieldimensionen für die technische, mathematische und informatische Bildung im Elementar- und Primarbereich erläutert. Diese Fachgrundlagen dienen der Stiftung als Fundierung für ihre Angebotsentwicklungen.

Seit 2019 geht die Stiftung mit verschiedenen Bildungsangeboten eigens für Lehrkräfte im unterrichtlichen Bereich auf die spezifischen Bedarfe dieser Zielgruppe ein und bietet damit ein Fortbildungsangebot an, das auf die Altersgruppe der Grundschulkinder ausgerichtet ist: Mit dem Projekt „Prima!Start“ (2019-2020, gefördert durch das Bundesministerium für Bildung und Forschung) widmete sich die Stiftung „Haus der kleinen Forscher“ der Entwicklung von Fortbildungsangeboten, die erstmals speziell an die Zielgruppe der Lehrkräfte im Grundschulunterricht gerichtet sind. Durch das Projekt wurden von März 2019 bis Juni 2020 die Grundlagen dafür geschaffen, über zielgruppenspezifische Fortbildungsangebote dabei mitzuwirken, die Kompetenzen von Grundschullehrkräften in den Domänen Fachwissen sowie Fachdidaktik und Pädagogik zu erweitern. Im Rahmen des Projektes wurden zwei Fortbildungsangebote zu den Themen „Energiebildung im Grundschulunterricht“ und „Informatische Bildung im Grundschulunterricht“ konzipiert.

Die Nutzung der Angebote befähigt die Lehrkräfte dazu, einen schülerorientierten Sachunterricht zu gestalten und die Aufbereitung der Themen an der Alltagswelt der Schülerinnen und Schüler auszurichten. Die Fortbildungen bringen die Lehrkräfte in Kontakt mit zeitgemäßen digitalen Lernangeboten mit dem Ziel, Nutzungshürden abzubauen und positive Nutzungserfahrungen zu ermöglichen. Aus diesem Grund wurde ein Blended-Learning-Format entwickelt. Die teilnehmenden Lehrkräfte erarbeiten in Kleingruppen Unterrichtsideen und diskutieren diese gemeinsam. So werden gemeinsam Konzepte entwickelt, die zu den individuellen Bedingungen an der Schule jeder einzelnen Lehrkraft passen. Die Referentinnen und Referenten vom „Haus der kleinen Forscher“ stehen ihnen dabei 
zur Seite und sind in engem Kontakt mit den Lernenden. Wie bereits beschrieben (siehe Abbildung 22) wechseln sich über die Dauer von zwölf Wochen aufeinander bezugnehmende Online- und Präsenzphasen ab, die es den Teilnehmenden ermöglichen, sich regelmäßig und zeitnah über ihre Praxiserfahrungen auszutauschen und diese zu reflektieren. Auch nach der Fortbildung steht ihnen die digitale Plattform für einen weiteren Austausch zur Verfügung.

Mit dem Projekt „Prima!2023“ (Laufzeit Juli 2020-Juni 2023) schließt die Stiftung an die Entwicklungen von „Prima!Start“ an. Das Projekt hat zum Ziel, das Fortbildungsangebot für Grundschullehrkräfte fachliche fundiert weiterzuentwickeln und um neue Angebote zu erweitern. Der besondere Schwerpunkt im Projekt „Prima!2023“ liegt demnach in der wissenschaftlich fundierten und wirkungsorientierten Weiterentwicklung der Blended-Learning-Fortbildungen zur Qualifizierung von Grundschullehrkräften in ganz Deutschland.

In der gemeinsam mit der Stiftung Lesen gegründeten Initiative „MINT- und Leseförderung“ (2019-2022, finanziert durch die Dieter Schwarz Stiftung) sammelt die Stiftung „Haus der kleinen Forscher“ ebenfalls Erfahrungen mit den spezifischen Bedarfen der Lehrkräfte bei der Gestaltung von pädagogischen Materialien für den Unterricht. Im Rahmen der Initiative entwickelten die beiden Stiftungen gemeinsam das Kindermagazin „echt jetzt?“ (Stiftung Lesen \& Stiftung Haus der kleinen Forscher, in Vorb.) für die dritte und vierte Klasse, von dem es bis Anfang 2022 vier Ausgaben geben wird. Das Magazin unterstützt Lehrkräfte dabei, mit den Kindern Lesen zu üben und mit ihnen im Unterricht zu forschen. Wie Lehrkräfte das Kindermagazin zielorientiert im Unterricht einsetzen können, erfahren sie praxisnah durch das Online-Fortbildungsangebot. Zusätzliche digitale Materialien unterstützen sie bei der Unterrichtsvorbereitung.

Des Weiteren entstanden im Rahmen des Projekts „innogy Digital-Lab“ (2016-2018, gefördert von der innogy Stiftung für Energie und Gesellschaft) zwei kostenfreie Lern-Apps für Kinder und ein pädagogischer Online-Kurs für das Selbststudium, um Grundschullehrkräfte in der Lernbegleitung zum Themenfeld „Strom und Energie“ zu unterstützen. Die Zielgruppe wurde durch Befragungen und Produkttests in die Angebotsentwicklung einbezogen. Die „Energie WimmelApp - Energie ist überall“ (siehe Web-App unter energie-wimmelapp.de) und die fürs Tablet optimierte App „Energie Schnitzeljagd - Energie ist überall“ (siehe Web-App unter energie-schnitzeljagd.de) ermöglichen Kindern einen intuitiven Einstieg ins Themenfeld und können ohne spezielle Vorkenntnisse genutzt werden.

In Rahmen des Folgeprojekts „Digital Lab 2.0, Wenn Lehrkräfte zu Entwicklern werden“(2019-2021, gefördert von der E.On Stiftung $\left.{ }^{26}\right)$ wird bereits an einer wei- 
teren App für den Grundschulunterricht gearbeitet. Doch dieses Mal wurden die Lehrkräfte selbst zu Entwicklerinnen und Entwicklern: Im Workshop-Angebot „Digitalwerkstatt Energie“ der Stiftung bekamen 22 Grundschullehrkräfte aus Berlin und der Region Hannover die Möglichkeit, eigenständig neue digitale Unterrichtstools für ihren Energiebildungsunterricht zu konzipieren. Die Stiftung erprobt in diesem Zusammenhang, wie ko-kreative Produktentwicklung bereits ab der Konzeption gelingen kann und welche Lerneffekte es bei den Mitwirkenden gibt. Mit Design-Thinking-Workshops, eigener Mitwirkung und der erforderlichen Infrastruktur unterstützte ein kleines Stiftungsteam die Lehrkräfte bei der Entwicklung von Produktideen und dem Basteln erster Prototypen. Aus insgesamt sechs entstandenen Ideen wurde Anfang 2020 schließlich eine ausgewählt: eine App, mit der sich Kinder ab der 3. Klasse interaktiv die Themen Stromerzeugung, Stromkreis und Energieeffizienz in unterschiedlichen Medienformen selbst erschließen können. Ein begleitendes Handbuch soll Lehrkräften, die die App im Unterricht einsetzen möchten, zudem Anregungen für die Verzahnung von On- und OfflineLernpfaden geben. Derzeit wird die App konzeptionell weiterentwickelt und mit einer professionellen Digitalagentur technisch und grafisch umgesetzt. Im Herbst 2020 wird ein Prototyp in diversen Grundschulen getestet. Ab Januar 2021 soll die neue App dann kostenfrei im Internet zur Verfügung stehen.

\section{Unterstützung der gesamten Bildungseinrichtung}

In externen Studien zur Wirkung der Fortbildungsangebote der Initiative „Haus der kleinen Forscher" sowie fachlichen Expertisen zu den Zielen der Fortbildungsangebote wurde die Bedeutung der systemischen Perspektive im Sinne eines „Whole Institution Approach“ (vgl. Ferreira, Ryan \& Tilbury, 2006) für die Verstetigung und Implementation früher MINT-Bildung im pädagogischen Alltag der Einrichtungen deutlich (vgl. Steffensky et al., 2018; Stiftung Haus der kleinen Forscher, 2019a). Ableitend aus den Ergebnissen der EASI-Science-Studie gab die Forschungsgruppe der Stiftung „Haus der kleinen Forscher“ u. a. mit auf den Weg, noch stärker als bislang systematisch auf Einrichtungsebene zu wirken und pädagogische Einrichtungen bei ihrer Qualitätsentwicklung zu begleiten (vgl. Stiftung Haus der kleinen Forscher, 2018b).

In der stiftungsinternen Längsschnittbefragung konnten diese Erkenntnisse bestätigt werden. Hier wurde der Zusammenhang der Kompetenzentwicklung von Pädagoginnen und Pädagogen nach dem Besuch einer „Haus der kleinen Forscher“-Präsenzfortbildung zu individuellen Eigenschaften als auch zu organisationalen Rahmenbedingungen betrachtet. Als organisationale Rahmenbedingungen konnten in der Untersuchungen die berufliche Funktion in der Einrichtung, die Verankerung von MINT-Bildungsinhalten in der Einrichtungskonzeption, der allgemeine Betreuungsschlüssel sowie der MINT-bezogene Betreuungsschlüssel 
berücksichtigt werden. Die Ergebnisse zeigen, dass insbesondere der MINT-bezogene Betreuungsschlüssel mit der Entwicklung im fachdidaktischen Wissen, der Motivation und der Selbstwirksamkeit zusammenhängt. Dies legt nahe, dass die organisationalen Rahmenbedingungen in den Einrichtungen für die dauerhafte Verstetigung von Kompetenzen bedeutsam sind. Wie genau Rahmenbedingungen der Einrichtungen mit Veränderungsprozessen und dem Transfer der Fortbildungsinhalte zusammenhängen, bedarf noch weiterer Untersuchungen.

Drei Projekte der Stiftung widmen sich derzeit auf unterschiedlichen Ebenen und mit unterschiedlichen Herangehensweisen der Qualitäts- und Organisationsentwicklung in Kitas. Sie nehmen die Bildungseinrichtung als Ganzesin den Blick und fördern die Verankerung des entdeckenden und forschenden Lernens im pädagogischen Alltag der Einrichtungen.

\section{Umsetzung in den Angeboten der Stiftung}

Mit der Erweiterung ihres Bildungsangebots um die Fortbildungen zum Bildungskonzept Bildung für nachhaltige Entwicklung (2016-2022, gefördert durch das Bundesministerium für Bildung und Forschung) hat sich die Stiftung bewusst einem systemorientierten Ansatz gewidmet. Insbesondere die Ansprache der Kita-Leitungen als spezifische Zielgruppe verfolgt das Ziel, auf der Systemebene der Einrichtungen wirksam zu werden. Deshalb wurden zum einen Fortbildungen für pädagogische Fachkräfte und zum anderen Fortbildungen für Kita-Leitungen entwickelt. Die Kita-Leitungen haben besondere Aufgaben und Anforderungen in ihrem Verantwortungsbereich, die spezifische Kompetenzen erfordern. Ihnen obliegt es, den organisatorischen Rahmen für die pädagogische Arbeit zu gestalten, Ressourcen bereitzustellen sowie eine langfristige Implementation von Bildung für nachhaltige Entwicklung in der Kita sicherzustellen. Die beiden Fortbildungsangebote „Tür auf! Mein Einstieg in Bildung für nachhaltige Entwicklung“ und „Macht mit! Bildung für nachhaltige Entwicklung in der Praxis“ für Kita-Leitungen thematisieren die für Einrichtungsleitungen wichtigen Aufgabenbereiche Strategie, pädagogische Führung, Management, Kommunikation und Selbstmanagement. Die Bereiche pädagogische Führung und Strategie werden vertieft adressiert, und die Leitungen erhalten insbesondere Impulse zu den Aufgabenbereichen Kommunikation und Management. In einem weiteren Fortbildungsangebot zu BNE für Kita-Leitungen sollen diese zukünftig insbesondere bei ihrer Aufgabe einer systematischen Qualitätsentwicklung unterstützt werden (Stiftung Haus der kleinen Forscher, 2019a).

Im Rahmen des Projekts „KiQ - gemeinsam für Kita-Qualität: Wenn Entdecken und Forschen zum Alltag werden“(2019-2022, gefördert vom Bundesministerium für Bildung und Forschung, s.o.) verstärkt die Stiftung künftig ihren Beitrag zur Qualitätsentwicklung von Kindertageseinrichtungen mit einem intensiven an- 
derthalbjährigen Kita-Programm. Mit diesem Fortbildungsprogramm zur alltagsintegrierten MINT-Bildung verfolgt die Stiftung einen systemorientierten Ansatz, der die Kompetenzentwicklung auf personenbezogener Ebene mit der bewussten Berücksichtigung von organisationalen Aspekte verschränkt. Dabei wird die Weiterentwicklung der Kita im Sinne einer Organisationsentwicklung besonders in den Blick genommen. Die „KiQ“-Programmaktivitäten zielen auf eine langfristige Verankerung des pädagogischen Ansatzes der Stiftung mittels einer bewussten Planung, Steuerung und Umsetzung eines einrichtungsspezifischen Veränderungsprozesses in den teilnehmenden Kitas ab. Hierbei sollen sowohl Maßnahmen auf der Ebene der Organisationskultur (z. B. gelebte Werte) als auch auf der Ebene der Organisationsstruktur (z. B. Kommunikationsstrukturen, Routinen und Abläufe) umgesetzt werden. Die Kita-Leitung soll dabei unterstützt werden, den Veränderungsprozess in ihrer Einrichtung so zu gestalten, dass dieser die Implementation des pädagogischen Ansatzes der Stiftung in den Arbeitsalltag fördert. Die Fachkraft wiederum soll mittels der fortlaufenden Qualifizierungsmaßnahmen unterstützt werden, das pädagogische Konzept in der Interaktion mit den Kindern umzusetzen. Zudem wird die Fachkraft in der Rolle einer Multiplikatorin beziehungsweise eines Multiplikators gestärkt, die/der das erworbene Wissen in einem nächsten Schritt mit den Teammitgliedern teilt und diese ebenfalls befähigt, das entdeckende und forschende Lernen im Alltag umzusetzen und so die Fragen aller Kinder der Einrichtung aufzugreifen und diesen nachzugehen.

Im Projekt „Forum KITA-Entwicklung“ (2019-2023, gefördert von der Robert Bosch Stiftung $\mathrm{GmbH}$ ) vertieft die Stiftung in Kooperation mit der Robert Bosch Stiftung die Frage, wie Organisationsentwicklung zur Qualitätsentwicklung in Kitas beitragen kann, aus theoretischer wie praktischer Perspektive.

Das Feld der Organisationsentwicklung in Kitas und deren Umwelt ist bisher kaum empirisch erforscht und beschrieben. Deshalb will das Forum KITA-Entwicklung Zusammenhänge zwischen Organisationsentwicklung und Qualitätsentwicklung auf Basis einer umfassenden Situationsanalyse und verschiedener wissenschaftlicher Expertise beschreiben und einen anhaltenden Austausch verschiedener Akteure aus Wissenschaft, Politik und Praxis über Veränderungsprozesse in Kindertageseinrichtungen etablieren. Es sucht nach übertragbaren Lösungsansätzen, die dazu beitragen, die Lernfähigkeit von Kitas als ganze Organisationen zu erhöhen, und für verschiedene thematische Bildungsbereiche nutzbar sind. Dazu sollen wirkungsorientierte Maßnahmen zur Unterstützung von Kita-Entwicklung entwickelt und exemplarisch erprobt, evaluiert sowie Handlungsempfehlungen für die Akteure im Feld abgeleitet werden. Die Ergebnisse werden für die Fach- und die bildungspolitische Diskussion aufbereitet und veröffentlicht. 


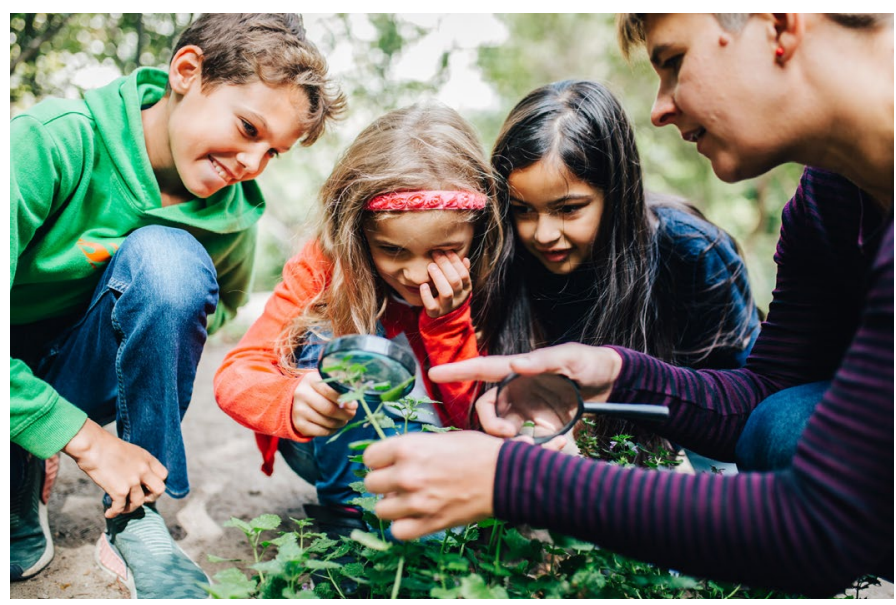

Das Forum KITA-Entwicklung strebt an, die verschiedenen Institutionen und Akteure miteinander ins Gespräch zu bringen, zu vernetzen und ihre Perspektiven zu bündeln. So soll ein gemeinsam erarbeitetes Verständnis entstehen, durch welche Prozesse und Strukturen pädagogische Qualität veränderbar ist. In einer Reihe von Fachgesprächen, Konferenzen und anderen Austauschformaten mit Kleingruppen suchen die Initiatoren des Forums KITA-Entwicklung den Dialog mit Akteurinnen und Akteuren aus allen Bereichen, die zum System der frühkindlichen Bildung gehören. Durch diesen Austausch soll ein umfassendes Bild und geteiltes Wissen entstehen, inwiefern Maßnahmen der Organisationsentwicklung zur Steigerung der Bildungsqualität in Einrichtungen der Frühpädagogik beitragen können. 


\section{Ausblick - weitere wissenschaftliche Begleitung und Erkenntnisbedarfe}

Zusammenfassend lässt sich festhalten, dass die beiden beschriebenen Untersuchungen erste Einblicke in den Professionalisierungsprozess von pädagogischen Fach- und Lehrkräften in der frühen MINT-Bildung ermöglicht haben. Die Stiftung wird in ihrer weiteren Angebotsentwicklung auf den Empfehlungen der „EpFL MINT“-Studie sowie den Schlussfolgerungen aus der stiftungsinternen Längsschnittbefragung aufbauen. Zusätzlich ist es aber von großer Bedeutung, weitere empirische Evidenz und weiterführende Erkenntnisse zu generieren und entsprechende Untersuchungen zu fördern, um mehr über den Professionalisierungsprozess von pädagogischen Fach-, Lehr- und Leitungskräften in der frühen MINT-Bildung zu erfahren. Insgesamt ist weitere Forschung notwendig, um gezielt die bedeutsamen individuellen Eigenschaften und organisationalen Rahmenbedingungen, die mit der professionellen Entwicklung der Pädagoginnen und Pädagogen zusammenhängen, sowie verschiedene Phasen des Professionalisierungsprozesses identifizieren zu können.

So werden beispielsweise in der Längsschnittbefragung auch situationsspezifische Vignetten zur Erfassung von alltagsnahem Verhalten eingesetzt und in zukünftigen Analysen ausgewertet, um eine Verzerrung durch Urteilsfehler wie bei der Selbsteinschätzung zu vermeiden. Eine Erfassung der MINT-bezogenen professionellen Kompetenzen von Pädagoginnen und Pädagogen anhand von Vignetten wird als vielversprechend angesehen, um Über- oder Unterschätzungen durch die pädagogischen Fach-, Lehr- und Leitungskräfte zu ihren professionellen Kompetenzen zu vermeiden (Brovelli et al., 2013). Außerdem ist nach weiteren Erhebungen eine Zeitreihenanalyse geplant, um Aussagen über langfristige Entwicklungen professioneller Kompetenzen treffen zu können. In zukünftigen Untersuchungen sollten mit Blick auf längerfristige Fortbildungseffekte weitere individuelle Eigenschaften und organisationale Rahmenbedingungen berücksichtigt werden, um tiefergehende Erkenntnisse über die bedeutenden, mit der Kompetenzentwicklung von Pädagoginnen und Pädagogen zusammenhängenden Merkmale zu gewinnen. Inhaltliche Schwerpunkte der nächsten geplanten Erhebung werden Bedingungen für den erfolgreichen Transfer von Fortbildungsinhalten in die pädagogische Praxis sowie förderliche und hemmende organisationale Rahmenbedingungen beim Transfer sein. Zudem wird das Spektrum der betrachteten Fortbildungsangebote sowohl um die neuentwickelte Präsenzfortbildung „MINT ist überall“ ergänzt als auch Teilnehmende von Online-Angeboten der Stiftung miteinbezogen. In künftigen Erhebungen werden außerdem höhere Fallzahlen angestrebt, indem die Stichprobe einerseits um neue Teilnehmende ergänzt wird 
und der Fragebogenrücklauf andererseits durch den parallelen Einsatz verschiedener Erhebungsmethoden erhöht werden soll.

Darüber hinaus wird die Stiftung weitere externe Wirkungsstudien fördern und auch eigene Wirkungsanalysen durchführen, um ihre Angebote wirkungsorientiert weiterzuentwickeln. Im Rahmen des „KiQ“-Projekts findet eine umfangreiche wissenschaftliche Begleitung statt, bestehend aus einer externen Begleitstudie, durchgeführt durch eine Forschungsgruppe der Eberhard Karls Universität Tübingen (wissenschaftlich verantwortlich: Prof. Caterina Gawrilow und Prof. Antje von Suchodoletz) und einer stiftungsinternen Evaluation. Ziel beider Untersuchungen ist es, Erkenntnisse darüber zu gewinnen, wie die Unterstützung der Verankerung eines pädagogischen Ansatzes im Einrichtungskonzept und der alltäglichen Praxis einer Einrichtung gelingen kann und damit wirkungsvolles Entdecken und Forschen im Alltag befördert werden können. Daraus möchte die Stiftung Erfolgsindikatoren für die Weiterentwicklung ihres Fortbildungsportfolios und eine mögliche Verstetigung des Konzepts in ihrem Qualifizierungsangebot ableiten.

Neben der Untersuchung der Wirkungen der „KiQ“-Programm-Interventionen auf individueller Ebene der Fachkraft und Kita-Leitung (wie Wissen, Fertigkeiten, Einstellungen, Transfer in die Praxis) werden auch Verbreitungsprozesse des pädagogischen Ansatzes zum entdeckenden und forschenden Lernen innerhalb des Kita-Teams analysiert. Des Weiteren wird der Frage nachgegangen, welche Veränderungen auf der Einrichtungsebene stattfinden und ob sich diese verstetigen lassen. Ebenso wird untersucht, welche organisationsinternen und -externen Faktoren einen fördernden oder hemmenden Einfluss auf den erfolgreichen Wissenstransfer ins Kita-Team und auf die Veränderungen auf der Organisationsebene entfalten.

Auch für das „Forum KITA-Entwicklung“ sind begleitende wissenschaftliche Untersuchungen geplant. Neben der Evaluation von entwickelten Maßnahmen zur Unterstützung von Organisationsentwicklung in Kitas sollen fachliche Expertisen nationale wie internationale Erkenntnisse zu theoretischen und empirischen $\mathrm{Zu}$ sammenhängen bündeln.

Seit 2011 können neben pädagogischen Fachkräften auch Lehrkräfte das Angebot der Stiftung „Haus der kleinen Forscher“ nutzen. Mit dem Bildungsprojekt „Sechs- bis zehnjährige Kinder“ schaffte die Stiftung im Sinne einer lückenlosen Bildungsbiografie einen fließenden Übergang für ihr Angebot. So können die Kinder ihre ersten Lernerfahrungen in Mathematik, Informatik, Naturwissenschaften und Technik im Anschluss an ihre Kita-Zeit vertiefen. Die Angebote, die im Rahmen dieses Projekts entstehen, sind für die Lernbegleitung im außerunterrichtlichen Bereich konzipiert.

Durch die bisherigen Erfahrungen der Stiftung mit der Zielgruppe der Lehrkräfte haben sich auch für den unterrichtlichen Bereich weiterführende Fragestel- 
lungen ergeben, zu denen die Stiftung in Zukunft vertiefende Erkenntnisse gewinnen möchte. Folgende Fragen sind dafür zentral:

Was ist guter MINT-Unterricht und wie sollte er gestaltet werden?

- Wie passt der pädagogische Ansatz der Stiftung „Haus der kleinen Forscher“ in den Unterricht?

- Wie muss ein Fortbildungsangebot der Stiftung für Grundschullehrkräfte im unterrichtlichen Bereich inhaltlich und methodisch beschaffen sein?

- Welche Fortbildungsformate (digital, Präsenz etc.) sind bei der Qualifizierung von Lehrkräften besonders wirkungsvoll?

Die Stiftung wird sich diesen Fragestellungen sowohl im Austausch mit wissenschaftlichen Fachexpertinnen und -experten der Grundschuldidaktik als auch anhand von Erkenntnissen aus der bisherigen Fachfundierung sowie eigenen Monitoring- und Evaluationsmaßnahmen zu den Angeboten für Lehrkräfte widmen. Ziel ist es, die Zielgruppe der Lehrkräfte und ihre Bedarfe im unterrichtlichen Bereich besser kennenzulernen und die Bildungsangebote darauf aufbauend (weiter-)zu entwickeln. Dafür geht die Stiftung auch in engen Austausch mit der Praxis und bindet diese beispielsweise über Fokusgruppen, Fachforen und die Entwicklung sowie Pilotierung von Materialien und Formaten in die (Weiter-)Entwicklung der Angebote für Lehrkräfte aktiv ein.

Insgesamt hat die Stiftung in den letzten Jahren viele (Weiter-)Entwicklungen auf dem Weg gebracht (u.a. in den Themenfeldern Kita-Entwicklung, Organisations- und Qualitätsentwicklung, Grundschule, Bildung für nachhaltige Entwicklung, Digitalisierung). Dabei wurde sie kontinuierlich von fachlichen Expertinnen und Experten und besonders durch den Wissenschaftlichen Beirat der Stiftung bei Forschungsfragen und der fachlichen Fundierung sowie der Wirkungsorientierung ihrer Angebote beraten. Aufbauend auf den Empfehlungen des Wissenschaftlichen Beirats entwickelt die Stiftung auch ihr Forschungskonzept kontinuierlich weiter und reagiert damit auf aktuelle Erkenntnisinteressen, die durch die stetige (Weiter-)Entwicklung ihrer Bildungsangebote entstehen. Neben dem weiteren Ausbau der stiftungsinternen Monitoring- und Evaluationsmaßnamen werden externe Begleitstudien im Rahmen der Projekte gefördert, Kontakte zur wissenschaftlichen Expertinnen und Experten in den entsprechenden Themenbereichen ausgebaut sowie nach weiteren Kooperationsmöglichkeiten für die wissenschaftliche Begleitung der Angebote gesucht.

Auch in Zukunft wird somit eine regelmäßige Reflexion und wirkungsorientierte (Weiter-)Entwicklung der Stiftungsangebote erfolgen. Die Bildungsinitiative „Haus der kleinen Forscher“ möchte damit wirkungsvoll zur Professionalisierung pädagogischer Fach- und Lehrkräfte beitragen. 


\section{Literatur}

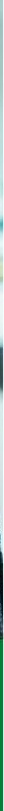




\section{Literatur}

\section{Geleitwort -}

\section{Mirjam Steffensky}

Leuchter, M. \& Saalbach, H. (2014). Verbale Unterstützungsmaßnahmen im Rahmen eines naturwissenschaftlichen Lernangebots in Kindergarten und Grundschule. Unterrichtswissenschaft, 42(2), 117-131.

Pedaste, M., Mäeots, M., Siiman, L. A., Jong, T. de, van Riesen, S. A., Kamp, E. T., Manoli, C. C., Zacharia, Z. C. \& Tsourlidaki, E. (2015). Phases of inquiry-based learning: Definitions and the inquiry cycle. Educational Research Review, 14, 47-61.

Piasta, S. B., Pelatti, C. Y. \& Miller, H. L. (2014). Mathematics and Science Learning Opportunities in Preschool Classrooms. Early Education and Development, 25(4), 445-468.

Sodian, B. \& Mayer, D. (2013). Entwicklung des wissenschaftlichen Denkens im Vor- und Grundschulalter. In M. Stamm \& D. Edelmann (Hrsg.), Handbuch frühkindliche Bildungsforschung (S. 617-631). Springer Fachmedien Wiesbaden.

White, B. Y. \& Frederiksen, J. R. (1998). Inquiry, Modeling, and Metacognition: Making Science Accessible to All Students. Cognition and Instruction, 16, 3-118.

\section{Einleitung -}

\section{Stiftung Haus der kleinen Forscher}

Anders, Y. \& Ballaschk, I. (2014). Studie zur Untersuchung der Reliabilität und Validität des Zertifizierungsverfahrens der Stiftung „Haus der kleinen Forscher“. In Stiftung Haus der kleinen Forscher (Hrsg.), Wissenschaftliche Untersuchungen zur Arbeit der Stiftung „Haus der kleinen Forscher“ (Band 6, S. 35-116). Schaffhausen: Schubi Lernmedien AG.

Arnold, M.-T., Carnap, A. \& Bormann, I. (2016). Bestandsaufnahme zur Verankerung von Bildung für nachhaltige Entwicklung in Bildungs- und Lehrplänen. Berlin: Stiftung Haus der kleinen Forscher.

Buschle, C. \& Gruber, V. (2018). Die Bedeutung von Weiterbildung für das Arbeitsfeld Kindertageseinrichtung. WIFF-Studien (Band 30). München: Weiterbildungsinitiative Frühpädagogische Fachkräfte.

Deutsche Telekom Stiftung \& Stiftung Haus der kleinen Forscher (in Vorb.). Lasst den Forschergeist frei! - Ausgezeichnete Projekte des Kita-Wettbewerbs Forschergeist 2020. Bonn: Deutsche Telekom Stiftung; Berlin: Stiftung Haus der kleinen Forscher. Verfügbar unter: www.haus-der-kleinen-forscher.de

Deutsches Jugendinstitut \& Weiterbildungsinitiative Frühpädagogische Fachkräfte (Hrsg.) (2014). Leitung von Kindertageseinrichtungen. Grundlagen für die kompetenzbasierte Weiterbildung. WiFF Wegweiser Weiterbildung (Band 10). München: Weiterbildungsinitiative Frühpädagogische Fachkräfte.

Friederich, T. (2019). Weiterbildung als zentrales Element einer nachhaltigen frühpädagogischen Professionalität? Eine professionstheoretisch-erwachsenenpädagogische Reflexion. Pädagogische Rundschau, 73(2), 165-176. 
Hippel, A. von (2011). Fortbildung in pädagogischen Berufen - zentrale Themen, Gemeinsamkeiten und Unterschiede der Fortbildung in Elementarbereich, Schule und Weiterbildung. In W. Helsper \& R. Tippelt, Pädagogische Professionalität (Zeitschrift für Pädagogik, Beiheft 57, S. 248-267). Weinheim: Beltz

König, A. \& Friederich, T. (Hrsg.) (2015). Qualität durch Weiterbildung. Konzeptionelle Denkanstöße für die Frühe Bildung. WIFF-Reihe: Perspektive Frühe Bildung (Band 3). Weinheim, Basel: Beltz Juventa.

Nationales MINT Forum (Hrsg.) (2018). Expertise zu Effekten zentraler außerschulischer MINT-Angebote. Berlin: Nationales MINT Forum e.V.

Rank, A., Wildemann, A., Pauen, S., Hartinger, A., Tietze, S. \& Kästner, R. (2018). Naturwissenschaftliche Bildung in der Kita: Gestaltung von Lehr-Lern-Situationen, sprachliche Anregungsqualität und sprachliche sowie naturwissenschaftliche Fähigkeiten der Kinder (EASI Science-L: Early Steps into Science and Literacy). In Stiftung Haus der kleinen Forscher (Hrsg.), Wirkungen naturwissenschaftlicher Bildungsangebote auf pädagogische Fachkräfte und Kinder. Wissenschaftliche Untersuchungen zur Arbeit der Stiftung „Haus der kleinen Forscher“ (Band 10, S. 138-251). Opladen, Berlin, Toronto: Verlag Barbara Budrich.

Starke, P. (2017). Frühpädagogische Professionalisierung - Das Ringen um Anerkennung als professionelles Selbst. In K. Jergus \& C. Thompson (Hrsg.), Autorisierungen des pädagogischen Selbst (S. 131-173). Wiesbaden: Springer Fachmedien.

Steffensky, M., Anders, Y., Barenthien, J., Hardy, I., Leuchter, M., Oppermann, E., Taskinen, P. \& Ziegler, T. (2018). Wirkungen früher naturwissenschaftlicher Bildungsangebote auf die naturwissenschaftlichen Kompetenzen von Fachkräften und Kindern (EASI Science: Early Steps into Science). In Stiftung Haus der kleinen Forscher (Hrsg.), Wirkungen naturwissenschaftlicher Bildungsangebote auf pädagogische Fachkräfte und Kinder. Wissenschaftliche Untersuchungen zur Arbeit der Stiftung „Haus der kleinen Forscher“ (Band 10, S. 50-136). Opladen, Berlin, Toronto: Verlag Barbara Budrich.

Stiftung Haus der kleinen Forscher (Hrsg.) (2013). Wissenschaftliche Untersuchungen zur Arbeit der Stiftung „Haus der kleinen Forscher“ (Band 5). Schaffhausen: Schubi Lernmedien AG. Verfügbar unter: www.haus-der-kleinen-forscher.de

Stiftung Haus der kleinen Forscher (Hrsg.) (2015). Wissenschaftliche Untersuchungen zur Arbeit der Stiftung „Haus der kleinen Forscher“ (Band 7). Schaffhausen: SCHUBI Lernmedien AG. Verfügbar unter: www.haus-der-kleinen-forscher.de

Stiftung Haus der kleinen Forscher (Hrsg.) (2017a). Frühe mathematische Bildung - Ziele und Gelingensbedingungen für den Elementar- und Primarbereich. Wissenschaftliche Untersuchungen zur Arbeit der Stiftung „Haus der kleinen Forscher“(Band 8). Opladen, Berlin, Toronto: Verlag Barbara Budrich. Verfügbar unter: www.haus-der-kleinen-forscher.de

Stiftung Haus der kleinen Forscher (2017b). Monitoring Bericht 2016/2017 der Stiftung „Haus der kleinen Forscher“. Berlin: Stiftung Haus der kleinen Forscher. Verfügbar unter: www.haus-der-kleinen-forscher.de

Stiftung Haus der kleinen Forscher (2017c). Zertifizierung für Kitas, Horte und Grundschulen. So wird ihre Einrichtung ein „Haus der kleinen Forscher“. Berlin: Stiftung Haus der kleinen Forscher. Verfügbar unter: www.haus-der-kleinen-forscher.de

Stiftung Haus der kleinen Forscher (Hrsg.) (2018a). Frühe informatische Bildung - Ziele und Gelingensbedingungen für den Elementar- und Primarbereich. Wissenschaftliche 
Untersuchungen zur Arbeit der Stiftung „Haus der kleinen Forscher“ (Band 9). Opladen, Berlin, Toronto: Verlag Barbara Budrich. Verfügbar unter: www.haus-der-kleinen-forscher.de

Stiftung Haus der kleinen Forscher (Hrsg.) (2018b). Wirkungen naturwissenschaftlicher Bildungsangebote auf pädagogische Fachkräfte und Kinder. Wissenschaftliche Untersuchungen zur Arbeit der Stiftung „Haus der kleinen Forscher“(Band 10). Opladen, Berlin, Toronto: Verlag Barbara Budrich. Verfügbar unter: www.haus-der-kleinen-forscher.de

Stiftung Haus der kleinen Forscher (Hrsg.) (2019a). Frühe Bildung für nachhaltige Entwicklung - Ziele und Gelingensbedingungen. Wissenschaftliche Untersuchungen zur Arbeit der Stiftung „Haus der kleinen Forscher“ (Band 12). Opladen, Berlin, Toronto: Verlag Barbara Budrich. Verfügbar unter: www.haus-der-kleinen-forscher.de

Stiftung Haus der kleinen Forscher (2019b). Pädagogischer Ansatz der Stiftung „Haus der kleinen Forscher" (6. Auflage). Berlin: Stiftung Haus der kleinen Forscher. Verfügbar unter: www.haus-der-kleinen-forscher.de

Stiftung Haus der kleinen Forscher (Hrsg.) (2019c). Zieldimensionen für Multiplikatorinnen und Multiplikatoren früher MINT-Bildung. Wissenschaftliche Untersuchungen zur Arbeit der Stiftung „Haus der kleinen Forscher“ (Band 11). Opladen, Berlin, Toronto: Verlag Barbara Budrich. Verfügbar unter: www.haus-der-kleinen-forscher.de

Stiftung Haus der kleinen Forscher (2020). Monitoring-Bericht 2018/2019 der Stiftung „Haus der kleinen Forscher“. Berlin: Stiftung Haus der kleinen Forscher. Verfügbar unter: www.haus-der-kleinen-forscher.de

Strehmel, P. \& Ulber, D. (Hrsg.) (2017). Kitas leiten und entwickeln: Ein Lehrbuch zum KitaManagement. Stuttgart: Kohlhammer Verlag.

\section{Entwicklungsverläufe von pädagogischen Fach- und Lehrkräften in der frühen MINT-Bildung -}

Nina Skorsetz, Lisa Öz, Julia Katharina Schmidt und Diemut Kucharz

Appleton, K. (2003). How Do Beginning Primary School Teachers Cope with Science? Toward an Understanding of Science Teaching Practice. Research in Science Education, 33(1), $1-25$.

Baumert, J. \& Kunter, M. (2006). Stichwort: Professionelle Kompetenz von Lehrkräften. Zeitschrift für Erziehungswissenschaft, 9(4), 469-520.

Blömeke, S., Kaiser, G. \& Lehmann, R. (Hrsg.) (2008). Professionelle Kompetenz angehender Lehrerinnen und Lehrer: Wissen, Überzeugungen und Lerngelegenheiten deutscher Mathematikstudierender und -referendare. Erste Ergebnisse zur Wirksamkeit der Lehrerausbildung. Münster: Waxmann.

Bosse, S., Henke, T., Jäntsch, C., Lambrecht, J., Vock, M. \& Spörer, N. (2016). Die Entwicklung der Einstellung zum inklusiven Lernen und der Selbstwirksamkeit von Grundschullehrkräften. Empirische Sonderpädagogik, 1, 103-116.

Brunner, M., Kunter, M., Krauss, S., Baumert, J., Blum, W., Dubberke, T., Jordan, A., Klusmann, U., Tsai, Y.-M. \& Neubrand, M. (2006). Welche Zusammenhänge bestehen zwischen dem fachspezifischen Professionswissen von Mathematiklehrkräften und ihrer Ausbildung sowie beruflichen Fortbildung? Zeitschrift für Erziehungswissenschaft, 9(4), 521-544. 
Bundesministerium für Bildung und Forschung (BMBF) (o.D.). Bildung. Frühe Förderung. Zugriff am 11.12.2018 unter https://www.bmbf.de/de/fruehe-foerderung-67.html

Döring, N. \& Bortz, J. (2016). Empirische Sozialforschung im Überblick. In N. Döring \& J. Bortz (Hrsg.), Forschungsmethoden und Evaluation in den Sozial- und Humanwissenschaften (S. 3-30). Berlin, Heidelberg: Springer.

Dunekacke, S. (2015). Erfassung mathematikdidaktischer Kompetenz von angehenden Erzieherinnen und Erziehern - Theoretische Überlegungen und methodisches Vorgehen. In J. Stiller \& C. Laschke (Hrsg.), Berlin-Brandenburger Beiträge zur Bildungsforschung. Herausforderungen, Befunde und Perspektiven interdisziplinärer Bildungsforschung (S. 106-121). Berlin: Lang.

Ernst, S. (2004). Angewandter „Methodenmix“? Gruppendiskussionen und schriftliche Befragungen am Beispiel eines Vorstudienmodells in der Hochschulevaluation. Forum Qualitative Sozialforschung, 5(2), Art. 3. Verfügbar unter: http://nbn-resolving.de/ urn:nbn:de:0114-fqs040231

Flick, U. (2006). Qualitative Sozialforschung. Eine Einführung. Reinbeck: Rowohlt Taschenbuchverlag.

Garbett, D. (2003). Science Education in Early Childhood Teacher Education: Putting Forward a Case to Enhance Student Teachers' Confidence and Competence. Research in Science Education, 33(4), 467-481.

Glynn, S. M. \& Koballa, T. R. Jr. (2006). Motivation to learn college science. In J. J. Mintzes \& W. H. Leonard (Hrsg.), Handbook of college science teaching (S. 25-32). Arlington, V.A: National Science Teachers Association Press.

Gold, A. \& Dubowy, M. (2013). Frühe Bildung - Lernförderung im Elementarbereich. Stuttgart: Kohlhammer Verlag.

Kauertz, A. \& Gierl, K. (2014). Naturwissenschaften im Elementarbereich. Analyse von Angeboten im naturwissenschaftlichen Bildungsbereich. In D. Kucharz, K. Mackowiak, S. Ziroli, A. Kauertz, E. Rathgeb-Schnierer \& M. Dieck (Hrsg.), Professionelles Handeln im Elementarbereich (PRIMEL). Eine deutsch-schweizerische Videostudie (S. 167-178). Münster: Waxmann.

Kucharz, D., Mackowiak, K., Dieck, M., Kauertz, A., Rathgeb-Schnierer, E. \& Ziroli, S. (2014). Theoretischer Hintergrund und aktueller Forschungsstand. In D. Kucharz, K. Mackowiak, S. Ziroli, A. Kauertz, E. Rathgeb-Schnierer \& M. Dieck (Hrsg.), Professionelles Handeln im Elementarbereich (PRIMEL): Eine deutsch-schweizerische Videostudie (S. 11-48). Münster: Waxmann.

Kunina-Habenicht, O., Decker, A.-T. \& Kunter, M. (2016). Lehrerpersönlichkeit und professionelle Kompetenz von Lehrkräften. In K. Seifried, S. Drewes \& M. Hasselhorn (Hrsg.), Handbuch Schulpsychologie: Psychologie für die Schule (S. 319-327). Stuttgart: Verlag W. Kohlhammer.

Kunter, M., Baumert, J., Blum, W., Klusmann, U., Krauss, S. \& Neubrand, M. (2011). Professionelle Kompetenz von Lehrkräften. Ergebnisse des Forschungsprogramms COACTIV. Münster: Waxmann.

Kunter, M., Baumert, J., Blum, W., Klusmann, U., Krauss, S. \& Neubrand, M. (2013). Cognitive Activation in the Mathematics Classroom and Professional Competence of Teachers: Results from the COACTIV Project. Mathematics Teacher Education. New York: Springer.

Kunter, M., Klusmann, U. \& Baumert, J. (2009). Professionelle Kompetenz von Mathematiklehrkräften: Das COACTIV-Modell. In O. Zlatkin-Troitschanskaia, K. Beck, D. Sembill, R. 
Nickolaus \& R. Mulder (Hrsg.), Lehrerprofessionalität. Bedingungen, Genese Wirkungen und ihre Messung. Weinheim, Basel: Beltz.

Lipowsky, F. \& Rzejak, D. (2012). Lehrerinnen und Lehrer als Lerner - Wann gelingt der Rollentausch? Merkmale und Wirkungen wirksamer Lehrerfortbildungen. Schulpädagogik heute, 3(5), 1-17.

Marquardt-Mau, B. (2004). Ansätze zur Scientific Literacy. Neue Wege für den Sachunterricht. In A. Kaiser \& D. Pech (Hrsg.), Neuere Konzeptionen und Zielsetzungen im Sachunterricht (S. 67-83). Hohengehren: Schneider Verlag.

Marquardt-Mau, B. (2011). Der Forschungskreislauf: Was bedeutet forschen im Sachunterricht? In Deutsche Telekom Stiftung und Deutsche Kinder- und Jugendstiftung (Hrsg.), Wie gute naturwissenschaftliche Bildung an Grundschulen gelingt. Ergebnisse und Erfahrungen aus prima(r)forscher (S. 37). Berlin: DKJS.

Mayring, P. (2001). Kombination und Integration qualitativer und quantitativer Analyse. Forum Qualitative Sozialforschung, 2(1), Art. 6. Verfügbar unter: http://nbn-resolving.de/ urn:nbn:de:0114-fqs010162

Mayring, P. (2002). Einführung in die qualitative Sozialforschung. Eine Anleitung zu qualitativem Denken. Weinheim: Beltz.

Morgan, P. L., Farkas, G., Hillemeier, M. H. \& Maczuga, S. (2016). Science Achievement Gaps Begin Very Early Persist and Are Largely Explained by Modifiable Factors. Educational Researcher, 45(1), 18-35.

Müllerbuchhof, R. \& Zehrt, P. (2006). Vergleich subjektiver und objektiver Messverfahren für die Bestimmung von Methodenkompetenz - am Beispiel der Kompetenzmessung bei technischem Fachpersonal. Zeitschrift für Arbeits- und Organisationspsychologie, 48, 132-138.

Myers, D. G. (2014). Sozialpsychologie. In D. G. Myers (Hrsg.), Psychologie (S. 595-653). Berlin, Heidelberg: Springer.

Neuweg, G. H. (2011). Das Wissen der Wissensvermittler. Problemstellungen, Befunde und Perspektiven der Forschung zum Lehrerwissen. In E. Terhart, H. Bennewitz \& M. Rothland (Hrsg.), Handbuch der Forschung zum Lehrerberuf (S. 451-477). Münster: Waxmann.

Nitsche, S., Dickhäuser, O., Dresel, M. \& Fasching, M. S. (2013). Zielorientierung von Lehrkräften als Prädiktoren lernrelevanten Verhaltens. Zeitschrift für Pädagogische Psychologie, 27, 95-103.

Rank, A., Wildemann, A., Hartinger, A. \& Tietze, S. (2018). Wirkungen sprachlicher Anregungsqualität in naturwissenschaftlichen Bildungsangeboten auf die sprachlichen Fähigkeiten von Vorschulkindern (EASI Science-L: Early Steps into Science and Literacy - Teilprojekt 1). In Stiftung Haus der kleinen Forscher (Hrsg.), Wirkungen naturwissenschaftlicher Bildungsangebote auf pädagogische Fachkräfte und Kinder. Wissenschaftliche Untersuchungen zur Arbeit der Stiftung „Haus der kleinen Forscher“ (Band 10, S. 140-193). Opladen, Berlin, Toronto: Verlag Barbara Budrich.

Richter, D. (2016). Lehrerinnen und Lehrer lernen: Fort- und Weiterbildung im Lehrerberuf. In M. Rothland (Hrsg.), Beruf Lehrer/Lehrerin: Ein Studienbuch (S. 245-260). Stuttgart: UTB.

Richter, E., Richter, D. \& Marx, A. (2018). Was hindert Lehrkräfte an Fortbildungen teilzunehmen? Eine empirische Untersuchung der Teilnahmebarrieren von Lehrkräften der Sekundarstufe I in Deutschland. Zeitschrift für Erziehungswissenschaften, 4, 1-23. 
Rost-Roth, M. (2001). Zweitsprachenerwerb als individueller Prozess IV: Affektive Variablen. In G. Helbig, L. Götze, G. Henrici \& H.-J. Krumm (Hrsg.), Deutsch als Fremdsprache: Band 19/1 (S. 714-722). Berlin, New York: De Gruyter.

Schäfer, G. E. (1995). Bildungsprozesse im Kindesalter: Selbstbildung, Erfahrung und Lernen in der frühen Kindheit. Weinheim, München: Juventa.

Schulz-Hardt, S., Rott, A., Meinken, I. \& Frey, D. (2001). Ein weiterentwickeltes Modell psychischer Sättigung. Psychologische Rundschau, 52(3), 141-149.

Steffensky, M., Anders, Y., Barenthien, J., Hardy, I., Leuchter, M., Oppermann, E., Taskinen, P. \& Ziegler, T. (2018). Wirkungen früher naturwissenschaftlicher Bildungsangebote auf die naturwissenschaftlichen Kompetenzen von Fachkräften und Kindern (EASI Science: Early Steps into Science). In Stiftung Haus der kleinen Forscher (Hrsg.), Wirkungen naturwissenschaftlicher Bildungsangebote auf pädagogische Fachkräfte und Kinder. Wissenschaftliche Untersuchungen zur Arbeit der Stiftung „Haus der kleinen Forscher“ (Band 10, S. 50-136). Opladen, Berlin, Toronto: Verlag Barbara Budrich.

Stiftung Haus der kleinen Forscher (Hrsg.) (2013). Wissenschaftliche Untersuchungen zur Arbeit der Stiftung „Haus der kleinen Forscher“(Band 5). Schaffhausen: SCHUBI Lernmedien AG. Verfügbar unter: www.haus-der-kleinen-forscher.de

Stiftung Haus der kleinen Forscher (2015a). Monitoring-Bericht 2015 der Stiftung „Haus der kleinen Forscher“. Berlin: Stiftung Haus der kleinen Forscher. Verfügbar unter: www. haus-der-kleinen-forscher.de

Stiftung Haus der kleinen Forscher (2015b). Pädagogischer Ansatz der Stiftung „Haus der kleinen Forscher“. Anregungen für die Lernbegleitung in Naturwissenschaften, Mathematik und Technik (5. Auflage). Berlin: Stiftung Haus der kleinen Forscher. Verfügbar unter: www.haus-der-kleinen-forscher.de

Stiftung Haus der kleinen Forscher (2017). Monitoring Bericht 2016/2017 der Stiftung „Haus der kleinen Forscher“. Berlin: Stiftung Haus der kleinen Forscher. Verfügbar unter: www.haus-der-kleinen-forscher.de

Stiftung Haus der kleinen Forscher (Hrsg.) (2018). Wirkungen naturwissenschaftlicher Bildungsangebote auf pädagogische Fachkräfte und Kinder. Wissenschaftliche Untersuchungen zur Arbeit der Stiftung „Haus der kleinen Forscher“(Band 10). Opladen, Berlin, Toronto: Verlag Barbara Budrich. Verfügbar unter: www.haus-der-kleinen-forscher.de

Stiftung Haus der kleinen Forscher (Hrsg.) (2019). Zieldimensionen für Multiplikatorinnen und Multiplikatoren früher MINT-Bildung. Wissenschaftliche Untersuchungen zur Arbeit der Stiftung „Haus der kleinen Forscher“(Band 11). Opladen, Berlin, Toronto: Verlag Barbara Budrich. Verfügbar unter: www.haus-der-kleinen-forscher.de

Vigerske, S. (2017). Transfer von Lehrerfortbildungsinhalten in die Praxis: Eine empirische Untersuchung zur Transferqualität und zu Einflussfaktoren. Wiesbaden: Springer.

Zimmermann, M. (2011). Naturwissenschaftliche Bildung im Kindergarten. Eine integrative Längsschnittstudie zur Kompetenzentwicklung von Erzieherinnen (Band 128). Berlin: Logos. 


\title{
B Kompetenzentwicklung von Pädagoginnen und Pädagogen im Längsschnitt -
}

\author{
Stiftung Haus der kleinen Forscher
}

Anders, Y. (2012). Modelle professioneller Kompetenzen für frühpädagogische Fachkräfte. Aktueller Stand und ihr Bezug zur Professionalisierung. Expertise zum Gutachten „Professionalisierung in der Frühpädagogik“im Auftrag des Aktionsrats Bildung (vbw - Vereinigung der Bayerischen Wirtschaft e. V., Hrsg.). München: KNOBLINGDESIGN GmbH.

Anders, Y. \& Ballaschk, I. (2014). Studie zur Untersuchung der Reliabilität und Validität des Zertifizierungsverfahrens der Stiftung „Haus der kleinen Forscher“. In Stiftung Haus der kleinen Forscher (Hrsg.), Wissenschaftliche Untersuchungen zur Arbeit der Stiftung „Haus der kleinen Forscher“ (Band 6, S. 35-116). Schaffhausen: Schaffhausen: Schubi Lernmedien AG.

Anders, Y., Hardy, I., Pauen, S. \& Steffensky, M. (2013). Zieldimensionen naturwissenschaftlicher Bildung im Kita-Alter und ihre Messung. In Stiftung Haus der kleinen Forscher (Hrsg.), Wissenschaftliche Untersuchungen zur Arbeit der Stiftung „Haus der kleinen Forscher" (Band 5, S. 19-82). Schaffhausen: Schaffhausen: Schubi Lernmedien AG.

Autorengruppe Fachkräftebarometer (2019). Fachkräftebarometer Frühe Bildung 2019. München: Weiterbildungsinitiative Frühpädagogische Fachkräfte.

Baumert, J. \& Kunter, M. (2011). Das Kompetenzmodell von COACTIV. In M. Kunter, J. Baumert, W. Blum, U. Klusmann, S. Krauss \& M. Neubrand (Hrsg.), Professionelle Kompetenz von Lehrkräften. Ergebnisse des Forschungsprogramms COACTIV (S. 29-54). Münster: Waxmann.

Benz, C., Grüßing, M., Lorenz, J. H., Selter, C. \& Wollring, B. (2017). Zieldimensionen mathematischer Bildung im Elementar- und Primarbereich. In Stiftung Haus der kleinen Forscher (Hrsg.), Frühe mathematische Bildung - Ziele und Gelingensbedingungen für den Elementar- und Primarbereich. Wissenschaftliche Untersuchungen zur Arbeit der Stiftung „Haus der kleinen Forscher“ (Band 8, S. 32-177). Opladen, Berlin, Toronto: Verlag Barbara Budrich.

Bergner, N., Köster, H., Magenheim, J., Müller, K., Romeike, R., Schroeder, U. \& Schulte, C. (2018). Zieldimensionen informatischer Bildung im Elementar- und Primarbereich. In Stiftung Haus der kleinen Forscher (Hrsg.), Frühe informatische Bildung - Ziele und Gelingensbedingungen für den Elementar- und Primarbereich. Wissenschaftliche Untersuchungen zur Arbeit der Stiftung „Haus der kleinen Forscher“ (Band 9, S. 38-267). Opladen, Berlin, Toronto: Verlag Barbara Budrich.

Bortz, J. \& Döring, N. (2006). Forschungsmethoden und Evaluation für Human- und Sozialwissenschaftler (4. Überarbeitete Auflage). Heidelberg: Springer Medizin Verlag.

Chin, W. W. (1998). The Partial Least Squares Approach to Structural Equation Modeling. In G.A. Marcoulides (Hrsg.), Modern Methods for Business Research (S. 295-336). Mahwah: Lawrence Erlbaum Associates.

Cohen, J. (1988). Statistical Power Analysis for the Behavioral Sciences (2. Auflage). Hillsdale, NJ: Lawrence Erlbaum Associates, Publishers.

Fröhlich-Gildhoff, K. (2014). Was bedeutet Kompetenzorientierung? ifp-Impulsfachtag „Kompetenzorientierung in der Weiterbildung“. Zugriff am 13.08.2019 unter: https:// www.ifp.bayern.de/imperia/md/content/stmas/ifp/impulsfachtage/impulsfachtag_ vortrag_froehlich.pdf 
Fröhlich-Gildhoff, K., Nentwig-Gesemann, I. \& Pietsch, S. (2011). Kompetenzorientierung in der Qualifizierung frühpädagogischer Fachkräfte. WiFF Expertisen (Band 19). München: DJI.

Fröhlich-Gildhoff, K., Weltzien, D., Kirstein, N., Pietsch, S. \& Rauh, K. (2014). Kompetenzen früh-/kindheitspädagogischer Fachkräfte im Spannungsfeld von normativen Vorgaben und Praxis. Expertise erstellt im Kontext der AG Fachkräftegewinnung für die Kindertagesbetreuung in Koordination des BMFSFJ, Berlin. Berlin: Bundesministerium für Familie, Senioren, Frauen und Jugend.

Kosack, W., Jeretin-Kopf, M. \& Wiesmüller, C. (2015). Zieldimensionen technischer Bildung im Elementar- und Primarbereich. In Stiftung Haus der kleinen Forscher (Hrsg.), Wissenschaftliche Untersuchungen zur Arbeit der Stiftung „Haus der kleinen Forscher“ (Band 7, S. 30-157). Schaffhausen: Schubi Lernmedien AG.

Rendtel, U. (1995). Lebenslagen im Wandel: Panelausfälle und Panelrepräsentativität. Frankfurt/Main, New York: Campus.

Schupp, J. (2014). Paneldaten für die Sozialforschung. In N. Baur \& J. Blasius (Hrsg.), Handbuch Methoden der empirischen Sozialforschung (S. 925-940). Wiesbaden: Springer Fachmedien.

Steffensky, M., Anders, Y., Barenthien, J., Hardy, I., Leuchter, M., Oppermann, E., Taskinen, P. \& Ziegler, T. (2018). Wirkungen früher naturwissenschaftlicher Bildungsangebote auf die naturwissenschaftlichen Kompetenzen von Fachkräften und Kindern (EASI Science: Early Steps into Science). In Stiftung Haus der kleinen Forscher (Hrsg.), Wirkungen naturwissenschaftlicher Bildungsangebote auf pädagogische Fachkräfte und Kinder. Wissenschaftliche Untersuchungen zur Arbeit der Stiftung „Haus der kleinen Forscher“ (Band 10, S. 50-136). Opladen, Berlin, Toronto: Verlag Barbara Budrich.

Stiftung Haus der kleinen Forscher (2009). Evaluationsbericht 2009. Berlin: Stiftung Haus der kleinen Forscher. Verfügbar unter: www.haus-der-kleinen-forscher.de

Stiftung Haus der kleinen Forscher (2010). Evaluationsbericht 2010. Berlin: Stiftung Haus der kleinen Forscher. Verfügbar unter: www.haus-der-kleinen-forscher.de

Stiftung Haus der kleinen Forscher (2011). Evaluationsbericht 2011 der Stiftung „Haus der kleinen Forscher“. Berlin: Stiftung Haus der kleinen Forscher. Verfügbar unter: www. haus-der-kleinen-forscher.de

Stiftung Haus der kleinen Forscher (2012). Evaluationsbericht 2012 der Stiftung „Haus der kleinen Forscher“ Berlin: Stiftung Haus der kleinen Forscher. Verfügbar unter: www. haus-der-kleinen-forscher.de

Stiftung Haus der kleinen Forscher (2013). Monitoring-Bericht 2013 der Stiftung „Haus der kleinen Forscher“. Berlin: Stiftung Haus der kleinen Forscher. Verfügbar unter: www. haus-der-kleinen-forscher.de

Stiftung Haus der kleinen Forscher (2014). Monitoring-Bericht 2014 der Stiftung „Haus der kleinen Forscher“. Berlin: Stiftung Haus der kleinen Forscher. Verfügbar unter: www. haus-der-kleinen-forscher.de

Stiftung Haus der kleinen Forscher (2015). Monitoring-Bericht 2015 der Stiftung „Haus der kleinen Forscher“. Berlin: Stiftung Haus der kleinen Forscher. Verfügbar unter: www. haus-der-kleinen-forscher.de

Stiftung Haus der kleinen Forscher (2017). Monitoring Bericht 2016/2017 der Stiftung „Haus der kleinen Forscher“. Berlin: Stiftung Haus der kleinen Forscher. Verfügbar unter: www.haus-der-kleinen-forscher.de 
Stiftung Haus der kleinen Forscher (2019). Pädagogischer Ansatz der Stiftung „Haus der kleinen Forscher" (6. Auflage). Berlin: Stiftung Haus der kleinen Forscher. Verfügbar unter: www.haus-der-kleinen-forscher.de

Stiftung Haus der kleinen Forscher (2020). Monitoring-Bericht 2018/2019 der Stiftung „Haus der kleinen Forscher“. Berlin: Stiftung Haus der kleinen Forscher. Verfügbar unter: www.haus-der-kleinen-forscher.de

Tietze, W. (Hrsg.) (1998). Wie gut sind unsere Kindergärten? Eine Untersuchung zur pädagogischen Qualität in deutschen Kindergärten. Weinheim: Beltz.

Weinert, F. E. (2001). Leistungsmessungen in Schulen. Weinheim und Basel: Beltz.

\section{Fazit und Ausblick -}

\section{Stiftung Haus der kleinen Forscher}

Anders, Y., Hardy, I., Pauen, S. \& Steffensky, M. (2013). Zieldimensionen naturwissenschaftlicher Bildung im Kita-Alter und ihre Messung. In Stiftung Haus der kleinen Forscher (Hrsg.), Wissenschaftliche Untersuchungen zur Arbeit der Stiftung „Haus der kleinen Forscher" (Band 5, S. 19-82). Schaffhausen: Schubi Lernmedien AG.

Anders, Y., Hardy, I., Sodian, B. \& Steffensky, M. (2013). Zieldimensionen naturwissenschaftlicher Bildung im Grundschulalter und ihre Messung. In Stiftung Haus der kleinen Forscher (Hrsg.), Wissenschaftliche Untersuchungen zur Arbeit der Stiftung „Haus der kleinen Forscher" (Band 5, S. 83-164). Schaffhausen: Schubi Lernmedien AG.

Bortz, J. \& Döring, N. (2006). Forschungsmethoden und Evaluation für Human- und Sozialwissenschaftler (4. überarbeitete Auflage). Heidelberg: Springer Medizin Verlag.

Brovelli, D., Bölsterli, K., Rehm, M. \& Wilhelm, M. (2013). Erfassen professioneller Kompetenzen für den naturwissenschaftlichen Unterricht: Ein Vignettentest mit authentisch komplexen Unterrichtssituationen und offenem Antwortformat. Unterrichtswissenschaft, 41(4), 306-329.

Ferreira, J., Ryan, L. \& Tilbury, D. (2006). Whole-School Approaches to Sustainability: A review of models for professional development in pre-service teacher education. Canberra: ARIES

Kunter, M. \& Klusmann, U. (2010). Kompetenzmessung bei Lehrkräften - Methodische Herausforderungen. Unterrichtswissenschaft, 38(1), 68-86.

Marquardt-Mau, B. (2004). Ansätze zur Scientific Literacy. Neue Wege für den Sachunterricht. In A. Kaiser \& D. Pech (Hrsg.), Neuere Konzeptionen und Zielsetzungen im Sachunterricht (S. 67-83). Hohengehren: Schneider Verlag.

Marquardt-Mau, B. (2011). Der Forschungskreislauf: Was bedeutet forschen im Sachunterricht? In Deutsche Telekom Stiftung und Deutsche Kinder- und Jugendstiftung (Hrsg.), Wie gute naturwissenschaftliche Bildung an Grundschulen gelingt. Ergebnisse und Erfahrungen aus prima(r)forscher (S. 37). Berlin: DKJS.

Ramseger, J. (2013). Prozessbezogene Qualitätskriterien für den naturwissenschaftlichen Unterricht - Zehn Kriterien für wirksames didaktisches Handeln im Elementar- und Primarbereich. In Stiftung Haus der kleinen Forscher (Hrsg.), Wissenschaftliche Untersuchungen zur Arbeit der Stiftung „Haus der kleinen Forscher“ (Band 5, S. 147-171). Schaffhausen: Schubi Lernmedien AG.

Rank, A., Wildemann, A., Hartinger, A. \& Tietze, S. (2018). Wirkungen sprachlicher Anregungsqualität in naturwissenschaftlichen Bildungsangeboten auf die sprachlichen Fä- 
higkeiten von Vorschulkindern (EASI Science-L: Early Steps into Science and Literacy - Teilprojekt 1). In Stiftung Haus der kleinen Forscher (Hrsg.), Wirkungen naturwissenschaftlicher Bildungsangebote auf pädagogische Fachkräfte und Kinder. Wissenschaftliche Untersuchungen zur Arbeit der Stiftung „Haus der kleinen Forscher“ (Band 10, S. 140-193). Opladen, Berlin, Toronto: Verlag Barbara Budrich.

Steffensky, M., Anders, Y., Barenthien, J., Hardy, I., Leuchter, M., Oppermann, E., Taskinen, P. \& Ziegler, T. (2018). Wirkungen früher naturwissenschaftlicher Bildungsangebote auf die naturwissenschaftlichen Kompetenzen von Fachkräften und Kindern (EASI Science: Early Steps into Science). In Stiftung Haus der kleinen Forscher (Hrsg.), Wirkungen naturwissenschaftlicher Bildungsangebote auf pädagogische Fachkräfte und Kinder. Wissenschaftliche Untersuchungen zur Arbeit der Stiftung „Haus der kleinen Forscher“ (Band 10, S. 50-136). Opladen, Berlin, Toronto: Verlag Barbara Budrich.

Stiftung Haus der kleinen Forscher (Hrsg.) (2013). Wissenschaftliche Untersuchungen zur Arbeit der Stiftung „Haus der kleinen Forscher“ (Band 5). SCHUBI Lernmedien AG. Verfügbar unter: www.haus-der-kleinen-forscher.de

Stiftung Haus der kleinen Forscher (Hrsg.) (2016). Tipps zur Lernbegleitung. Karten-Set für Kinder „Zahlen, Zählen, Rechnen - Mathematik entdecken“. Berlin: Stiftung Haus der kleinen Forscher. Verfügbar unter: www.haus-der-kleinen-forscher.de

Stiftung Haus der kleinen Forscher (2018a). Kinderkarten Technik - von hier nach da. Berlin: Stiftung Haus der kleinen Forscher. Verfügbar unter: www.haus-der-kleinen-forscher. de

Stiftung Haus der kleinen Forscher (Hrsg.) (2018b). Wirkungen naturwissenschaftlicher Bildungsangebote auf pädagogische Fachkräfte und Kinder (Band 10). Opladen, Berlin, Toronto: Verlag Barbara Budrich. Verfügbar unter: www.haus-der-kleinen-forscher.de

Stiftung Haus der kleinen Forscher (Hrsg.) (2019a). Frühe Bildung für nachhaltige Entwicklung - Ziele und Gelingensbedingungen (Band 12). Opladen, Berlin, Toronto: Verlag Barbara Budrich. Verfügbar unter: www.haus-der-kleinen-forscher.de

Stiftung Haus der kleinen Forscher (2019b). Materialpaket „MINT ist überall“. Berlin: Stiftung Haus der kleinen Forscher.

Stiftung Haus der kleinen Forscher (2019c). MINT ist überall. Berlin: Stiftung Haus der kleinen Forscher. Verfügbar unter: www.haus-der-kleinen-forscher.de

Stiftung Haus der kleinen Forscher (2019d). Pädagogischer Ansatz der Stiftung „Haus der kleinen Forscher“ (6. Auflage). Berlin: Stiftung Haus der kleinen Forscher. Verfügbar unter: www.haus-der-kleinen-forscher.de

Stiftung Lesen \& Stiftung Haus der kleinen Forscher (Hrsg.) (in Vorb.). echt jetzt? Das Wetter. Mainz, Berlin: Stiftung Lesen und Stiftung Haus der kleinen Forscher. 



\section{Anhang}

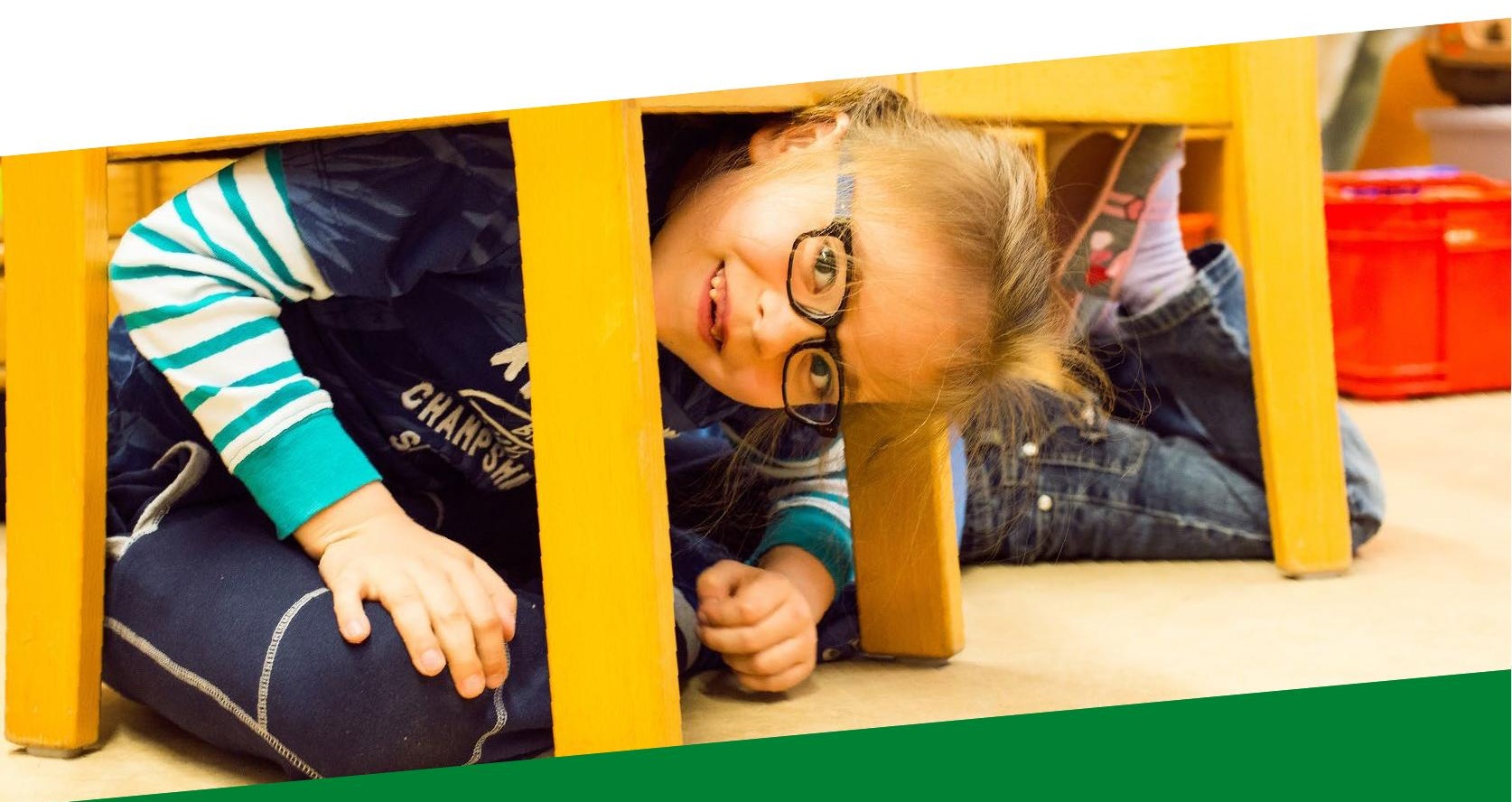


Anhang

Kategorien- und Kodiersystem der Interviewauswertung 


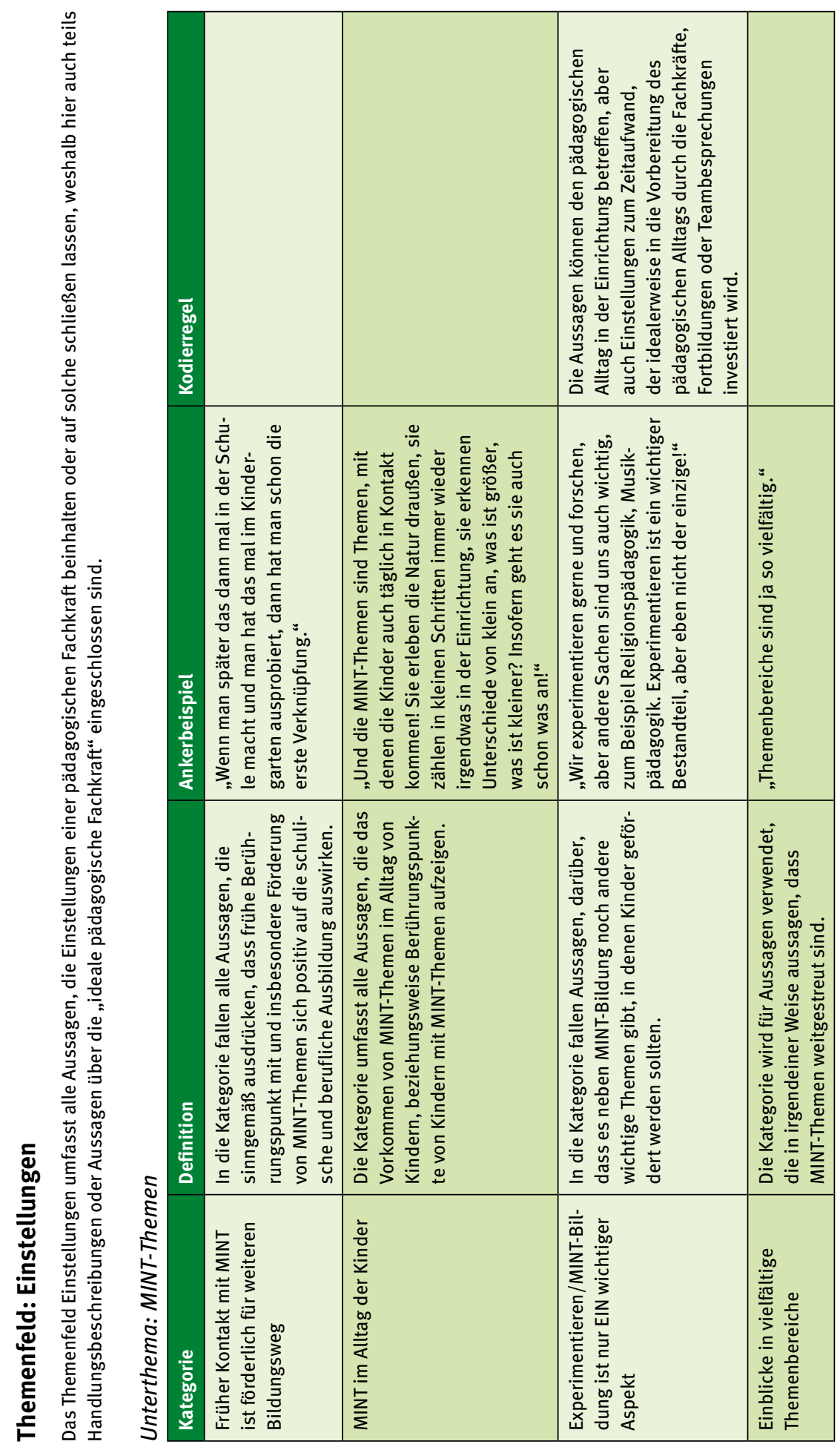




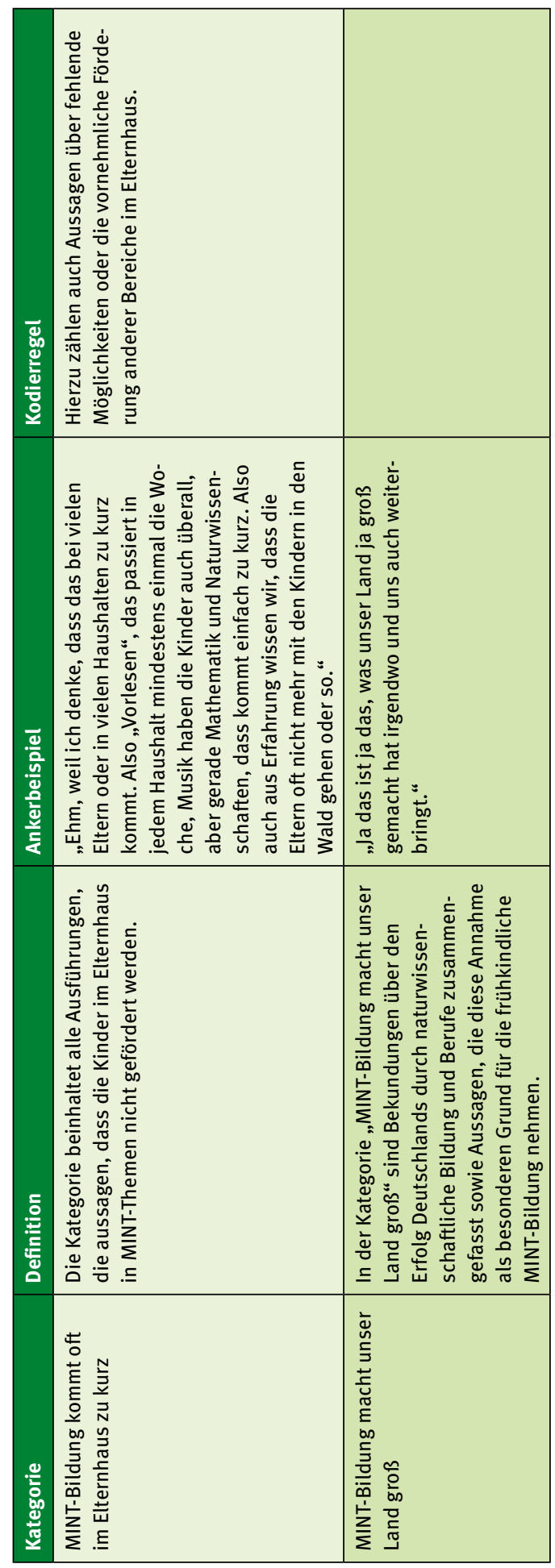




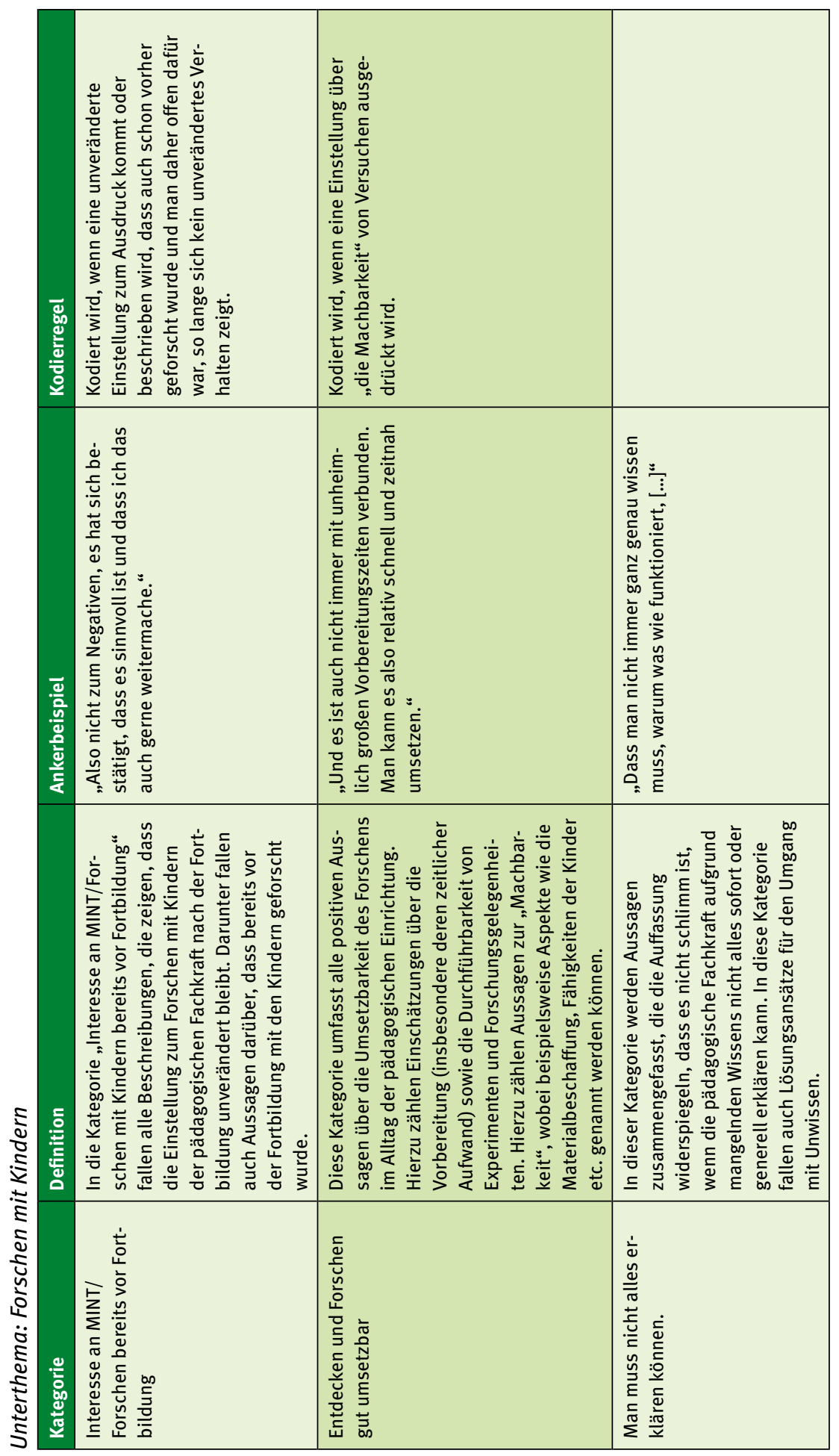




\begin{tabular}{|c|c|c|c|c|}
\hline & 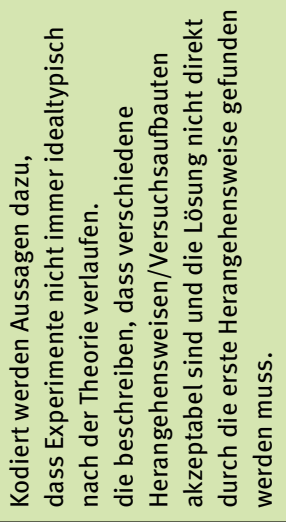 & 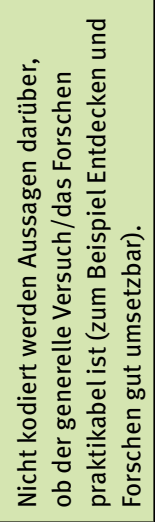 & & \\
\hline & 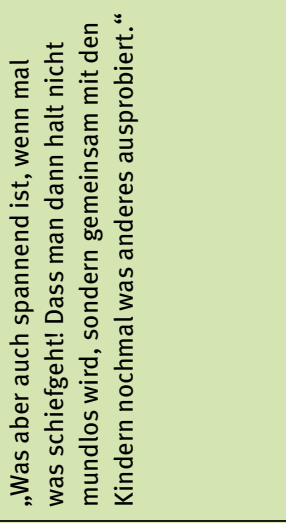 & & 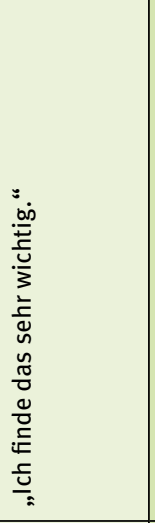 & 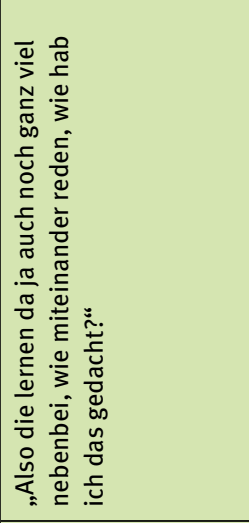 \\
\hline & 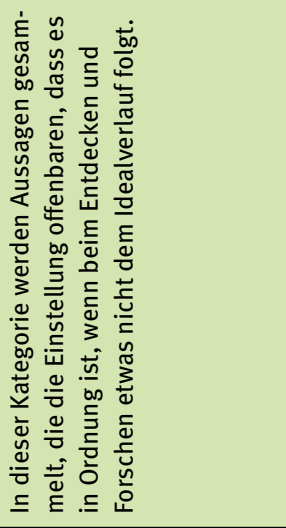 & & 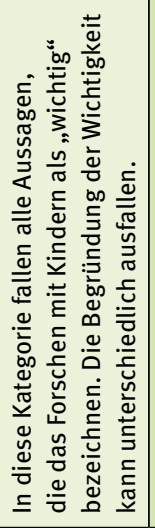 & 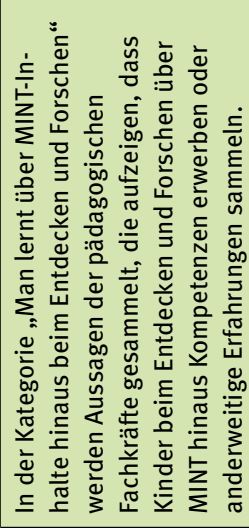 \\
\hline 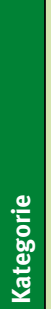 & 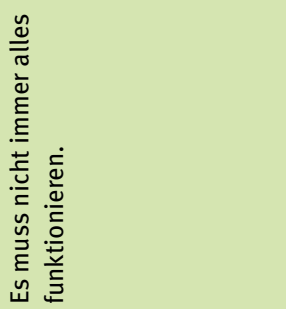 & & 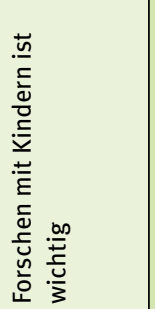 & 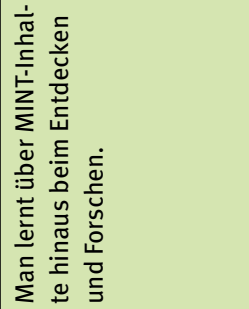 \\
\hline
\end{tabular}




\begin{tabular}{|c|c|c|c|c|}
\hline & & 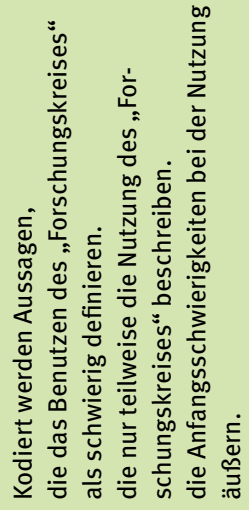 & & \\
\hline & & 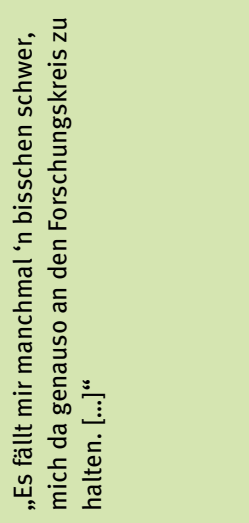 & 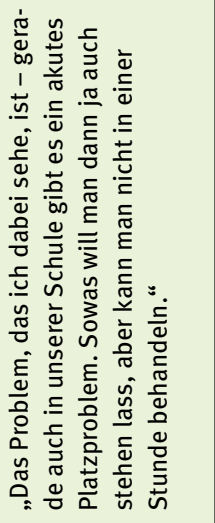 & 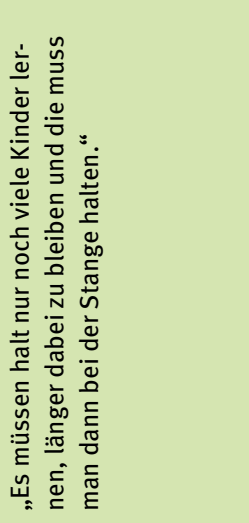 \\
\hline & 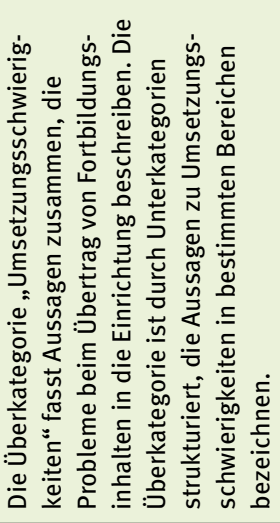 & 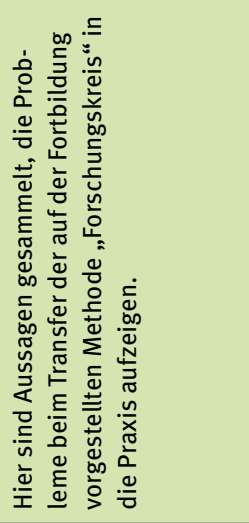 & 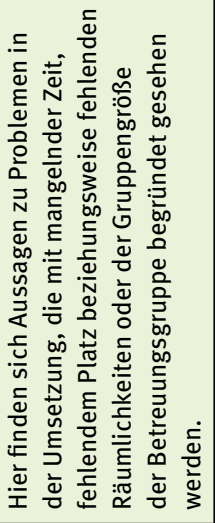 & 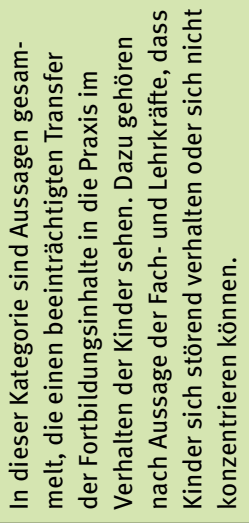 \\
\hline & 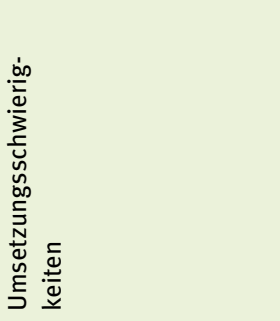 & 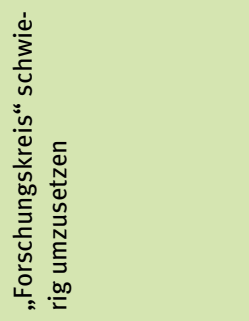 & 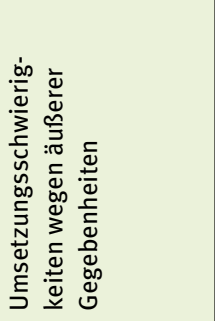 & 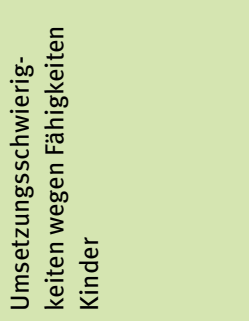 \\
\hline
\end{tabular}




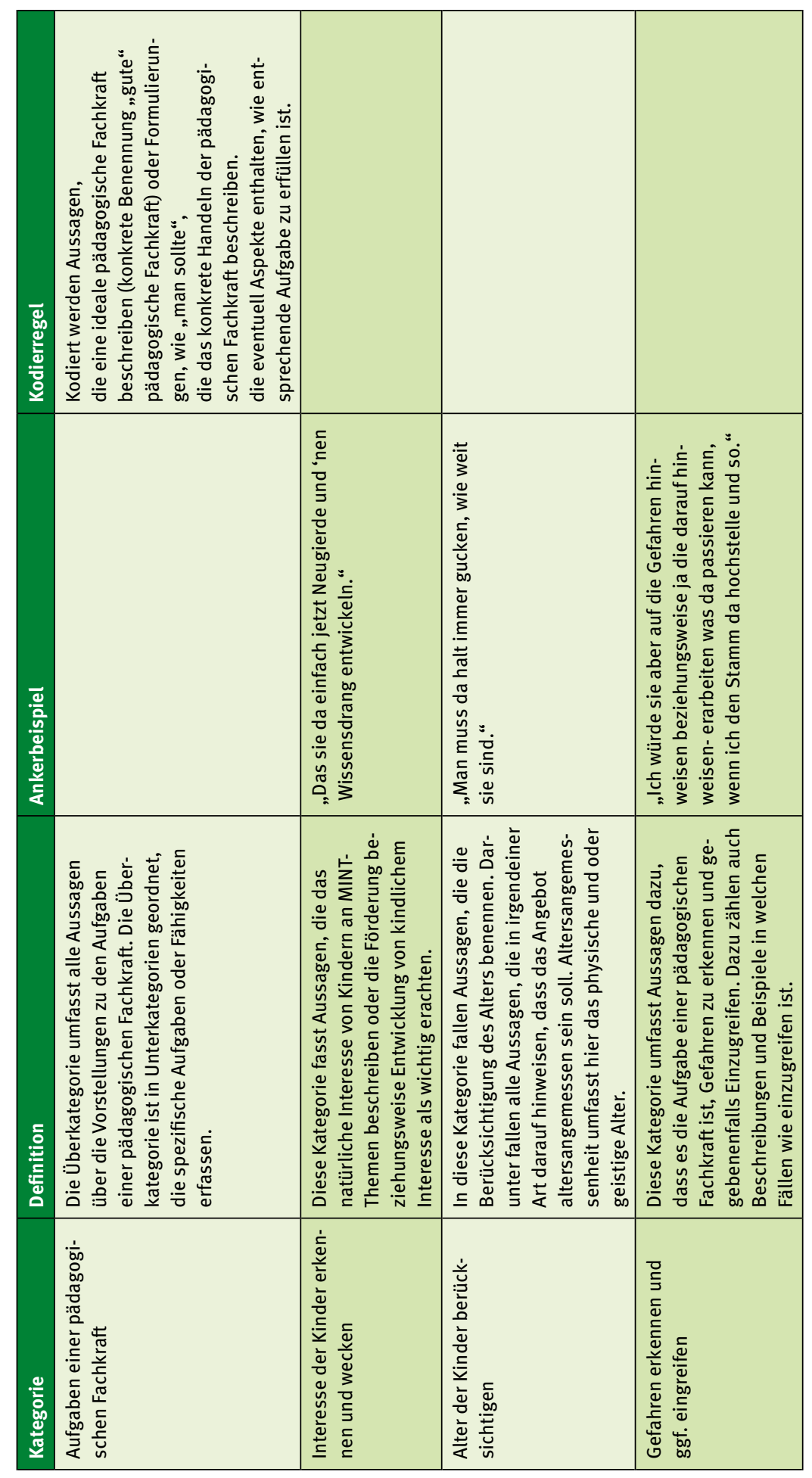




\begin{tabular}{|c|c|c|c|c|}
\hline & & 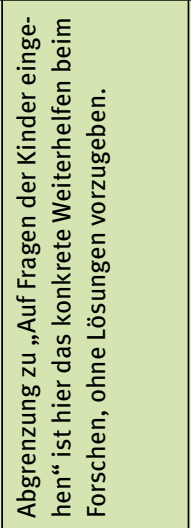 & 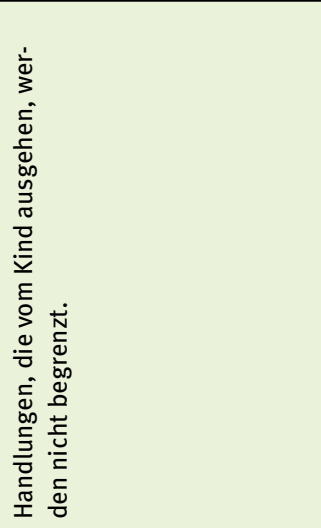 & 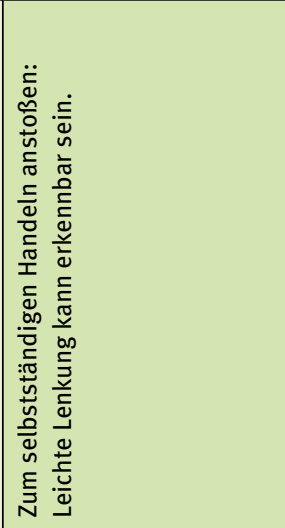 \\
\hline & 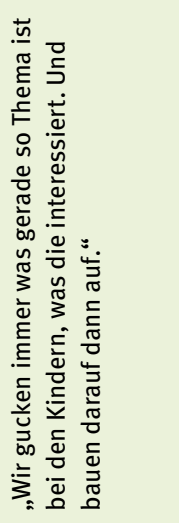 & 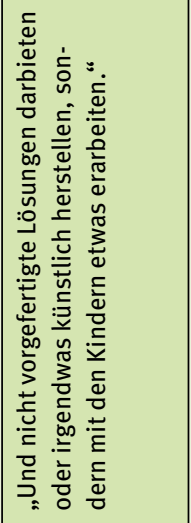 & 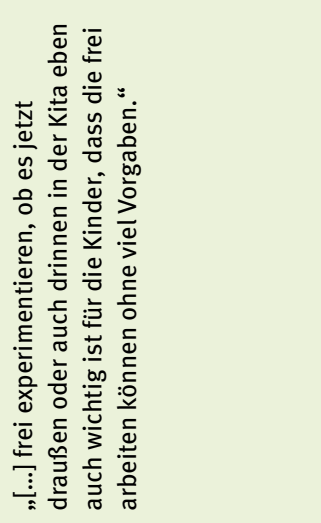 & 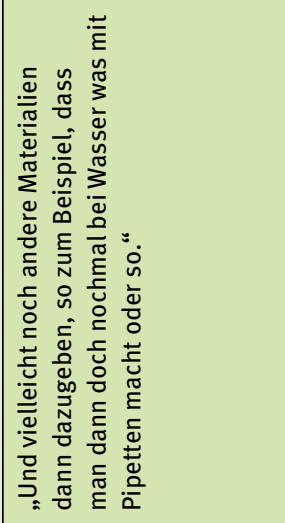 \\
\hline & 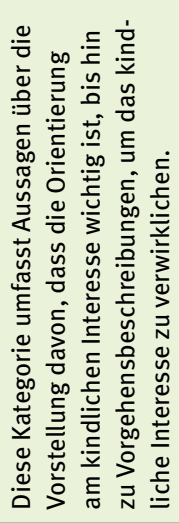 & 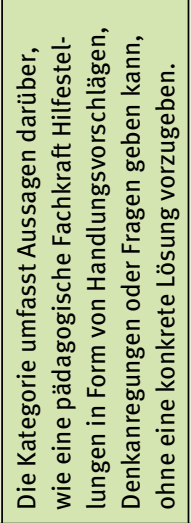 & 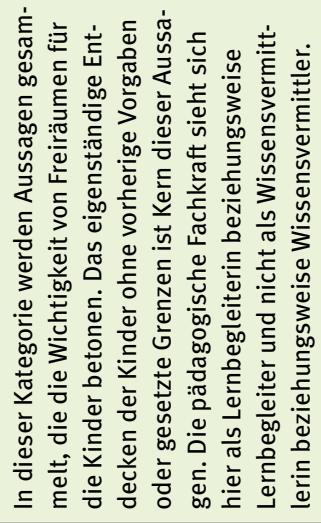 & 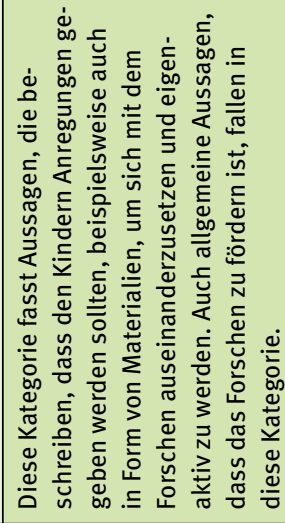 \\
\hline & 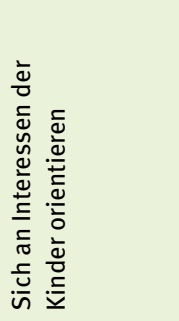 & 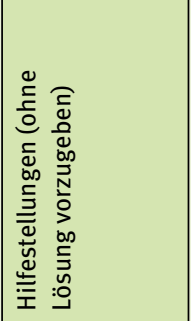 & 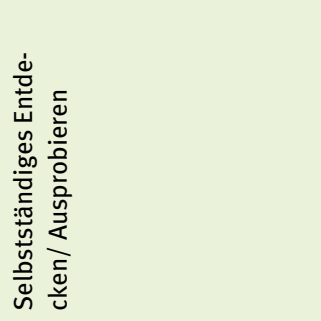 & 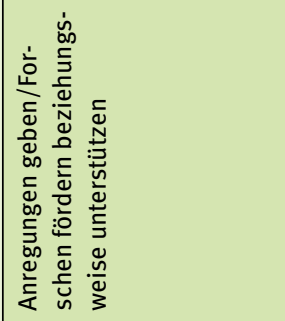 \\
\hline
\end{tabular}




\begin{tabular}{|c|c|c|c|c|}
\hline & 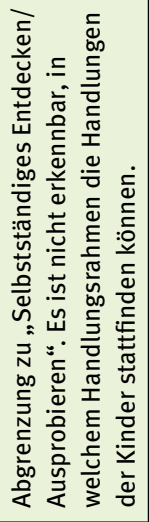 & & 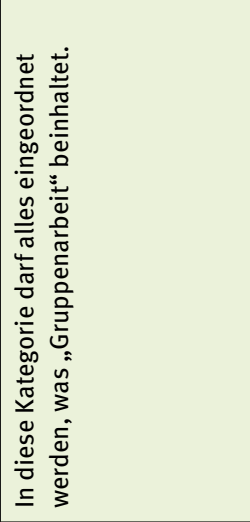 & 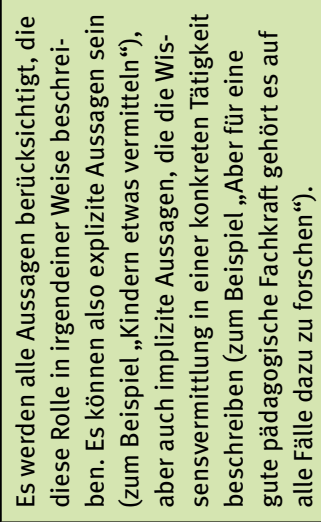 \\
\hline & 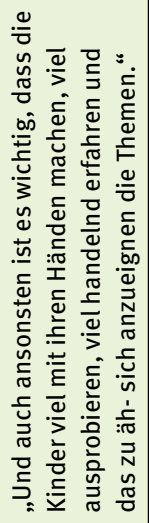 & 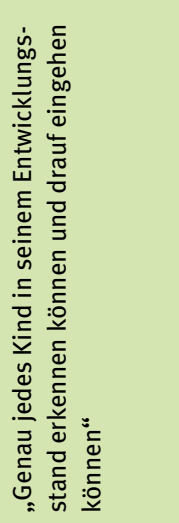 & 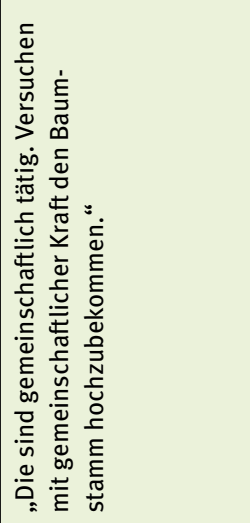 & 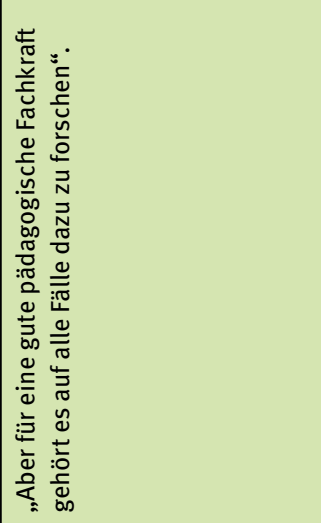 \\
\hline 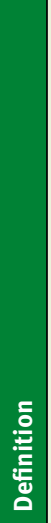 & 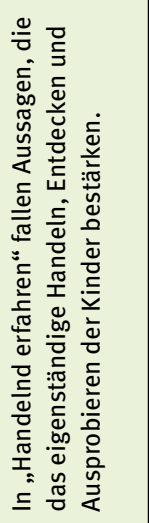 & 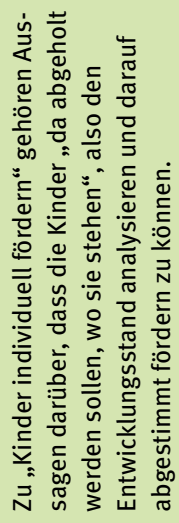 & 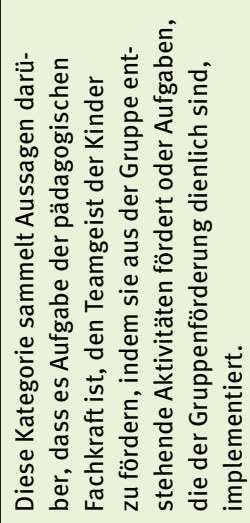 & 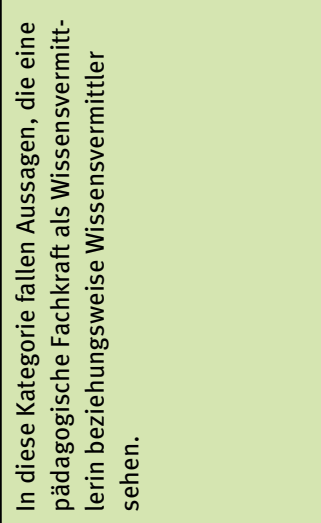 \\
\hline & 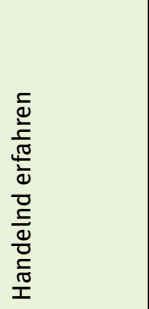 & 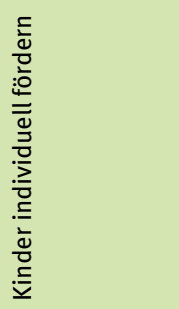 & 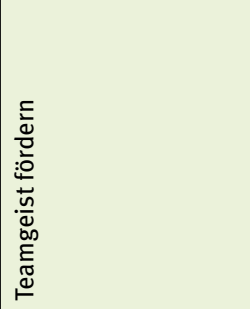 & 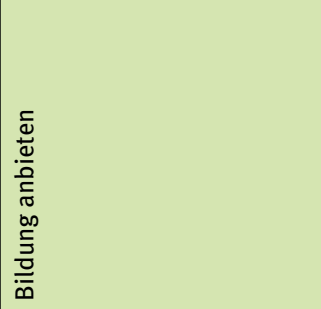 \\
\hline
\end{tabular}



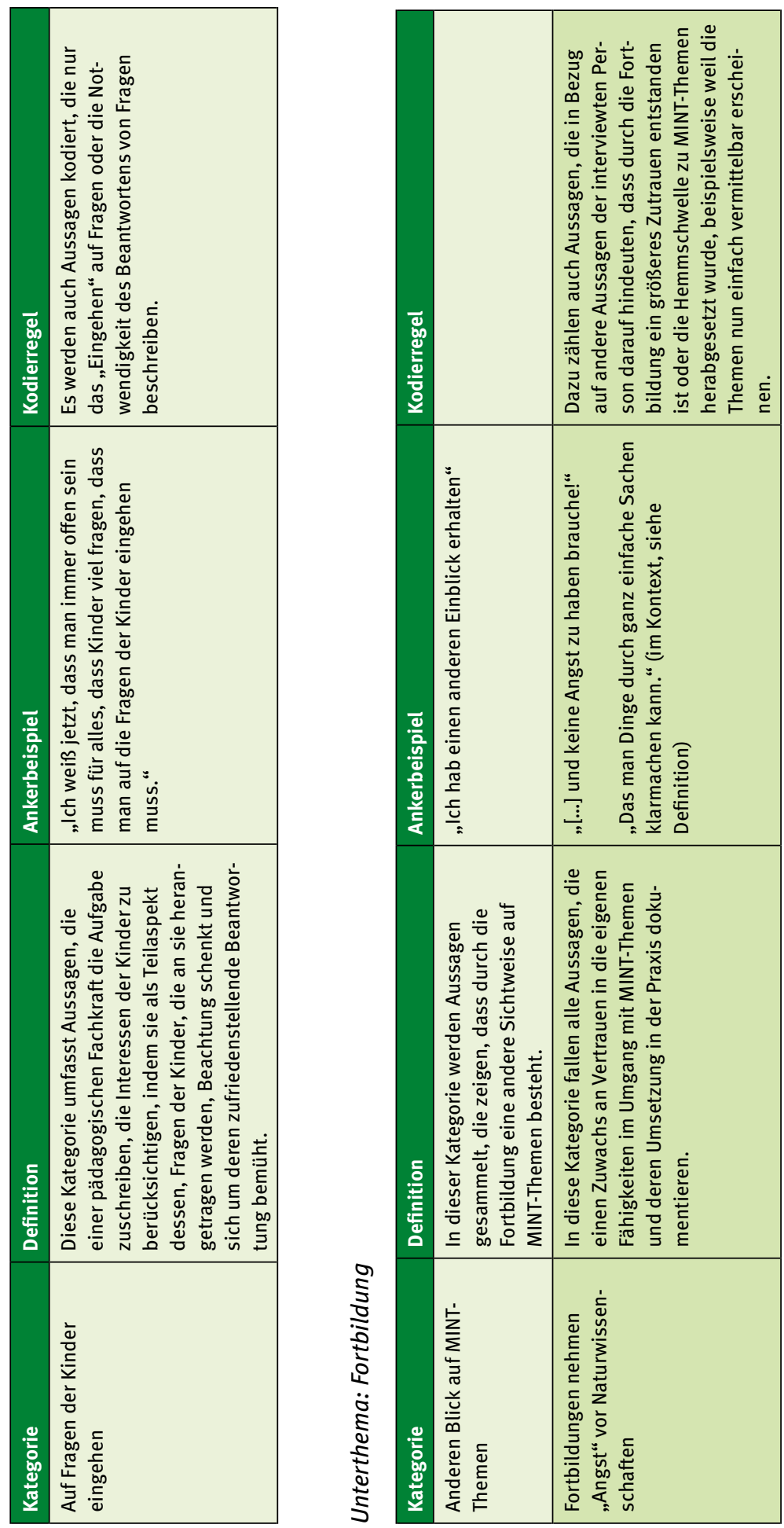


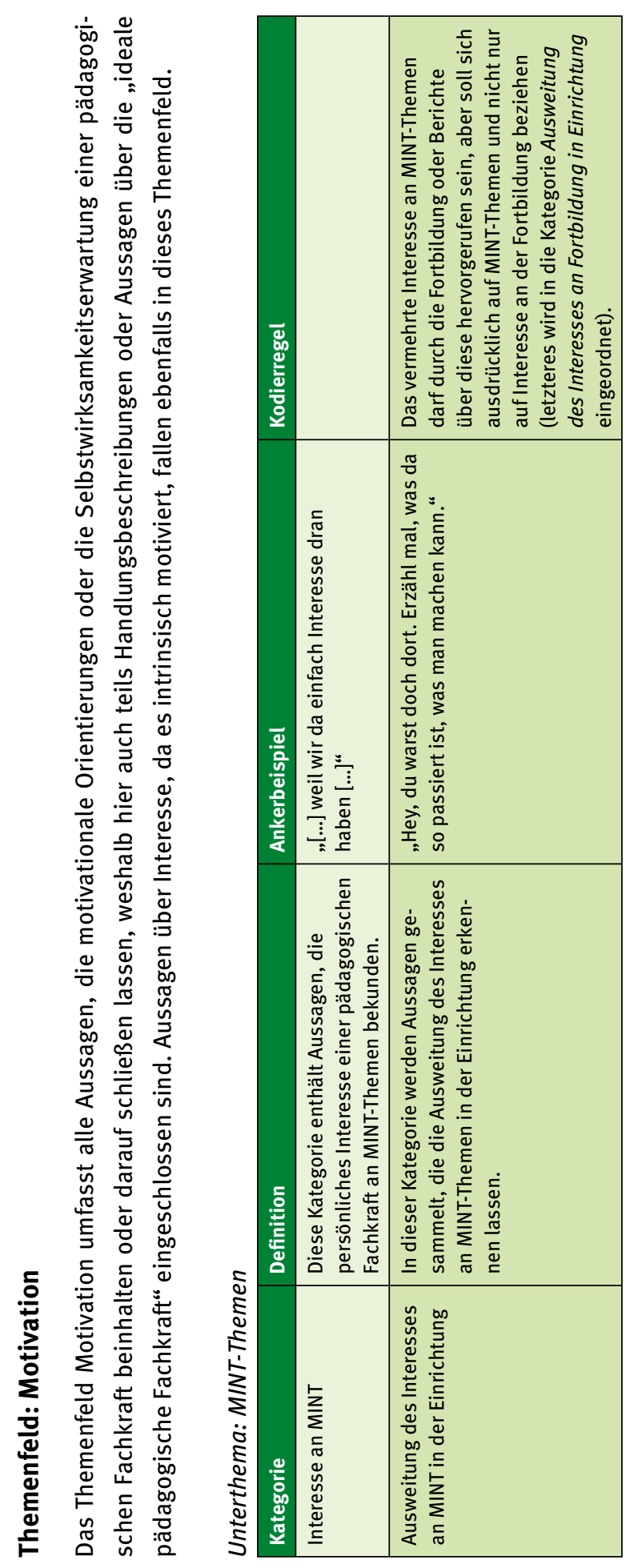




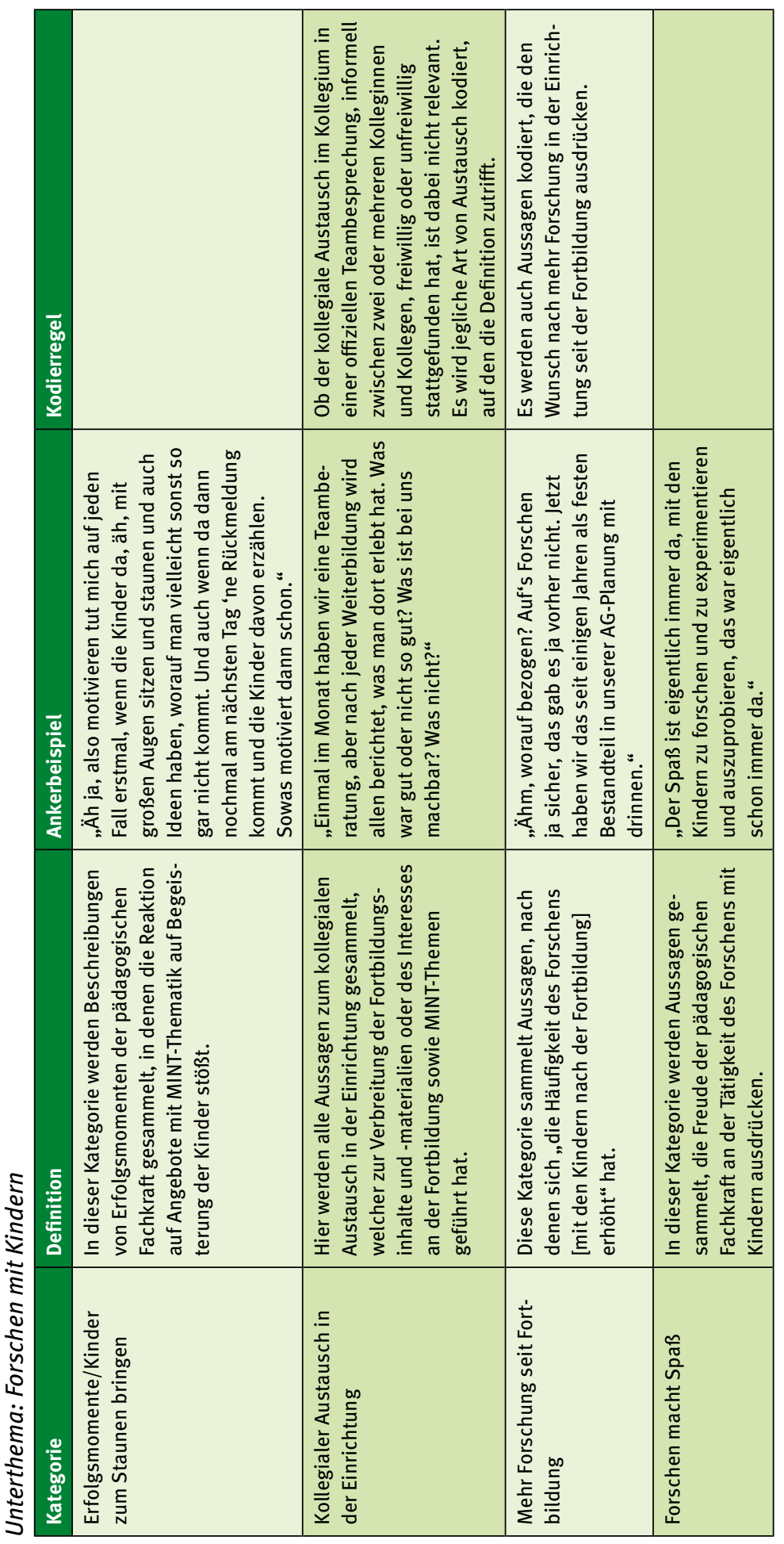




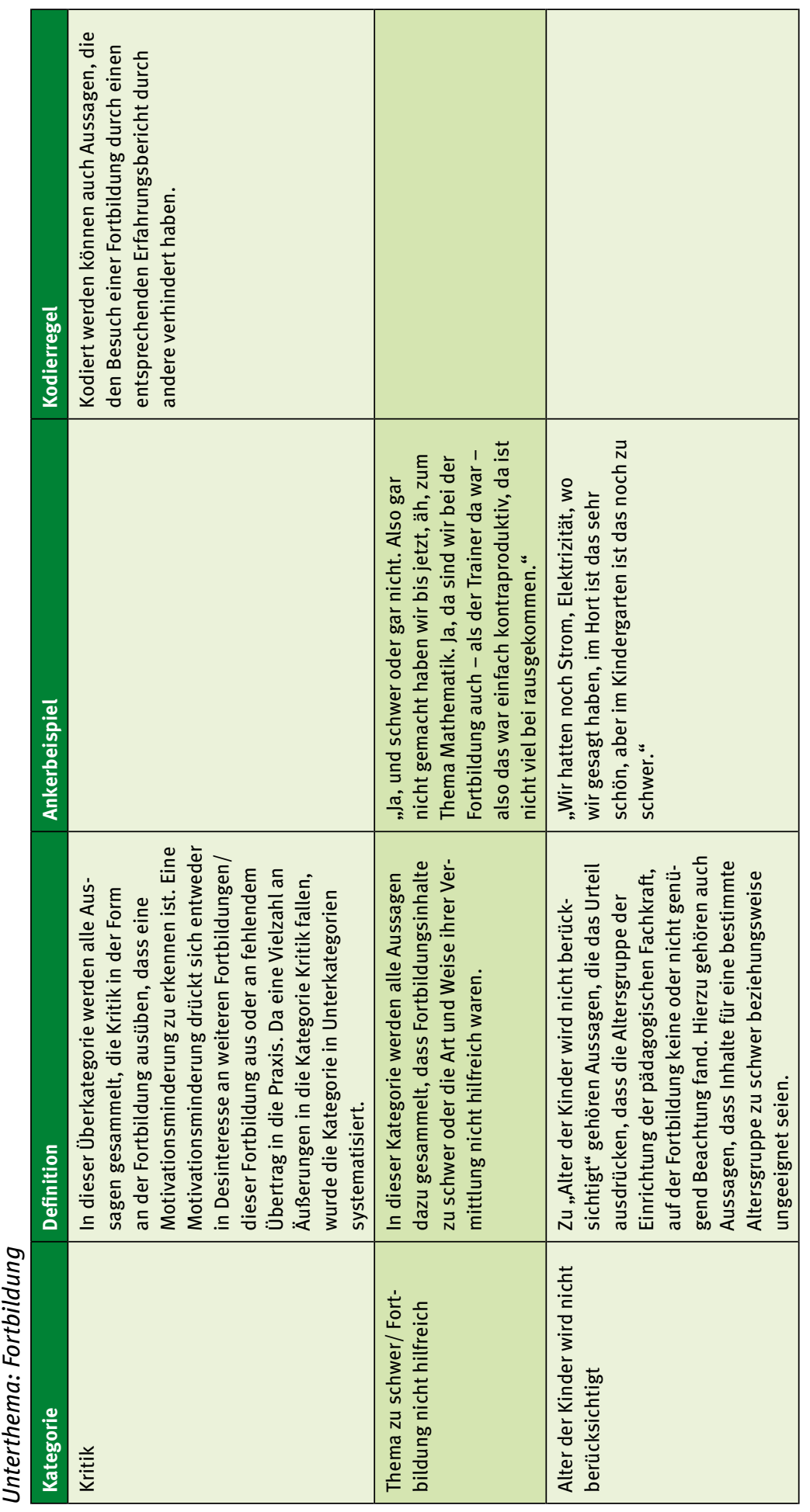




\begin{tabular}{|c|c|c|c|c|}
\hline & & 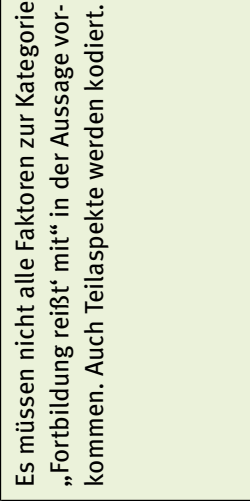 & 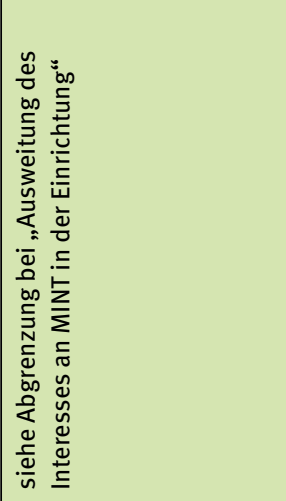 & \\
\hline & 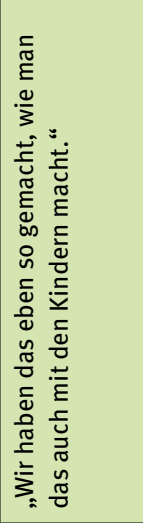 & 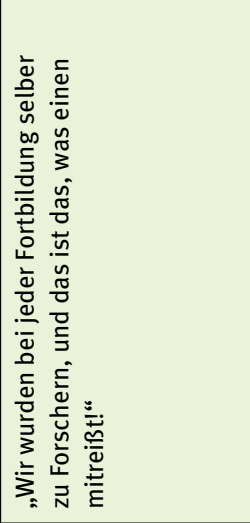 & 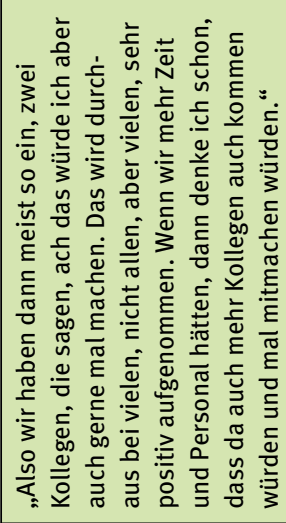 & 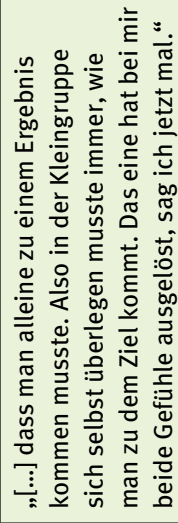 \\
\hline 흘 & 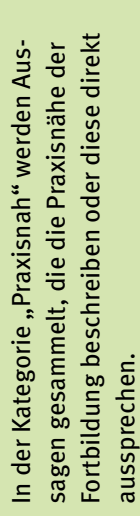 & 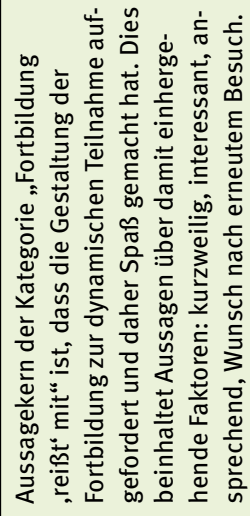 & 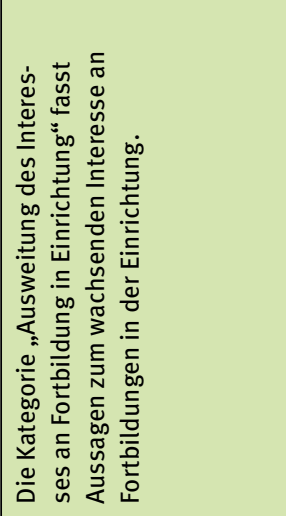 & 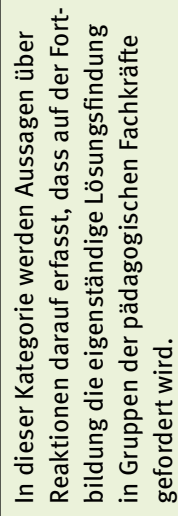 \\
\hline & $\begin{array}{l}\frac{\pi}{5} \\
\frac{n}{x} \\
\frac{\pi}{2} \\
\frac{\pi}{2}\end{array}$ & 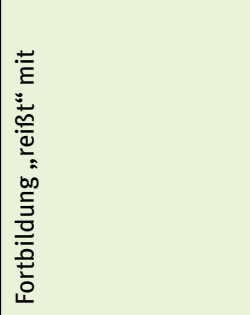 & 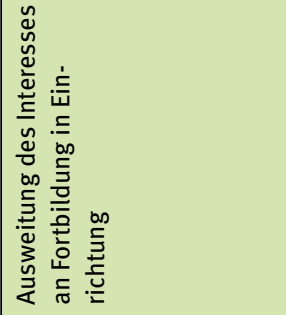 & 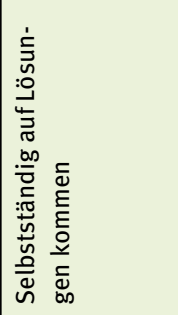 \\
\hline
\end{tabular}




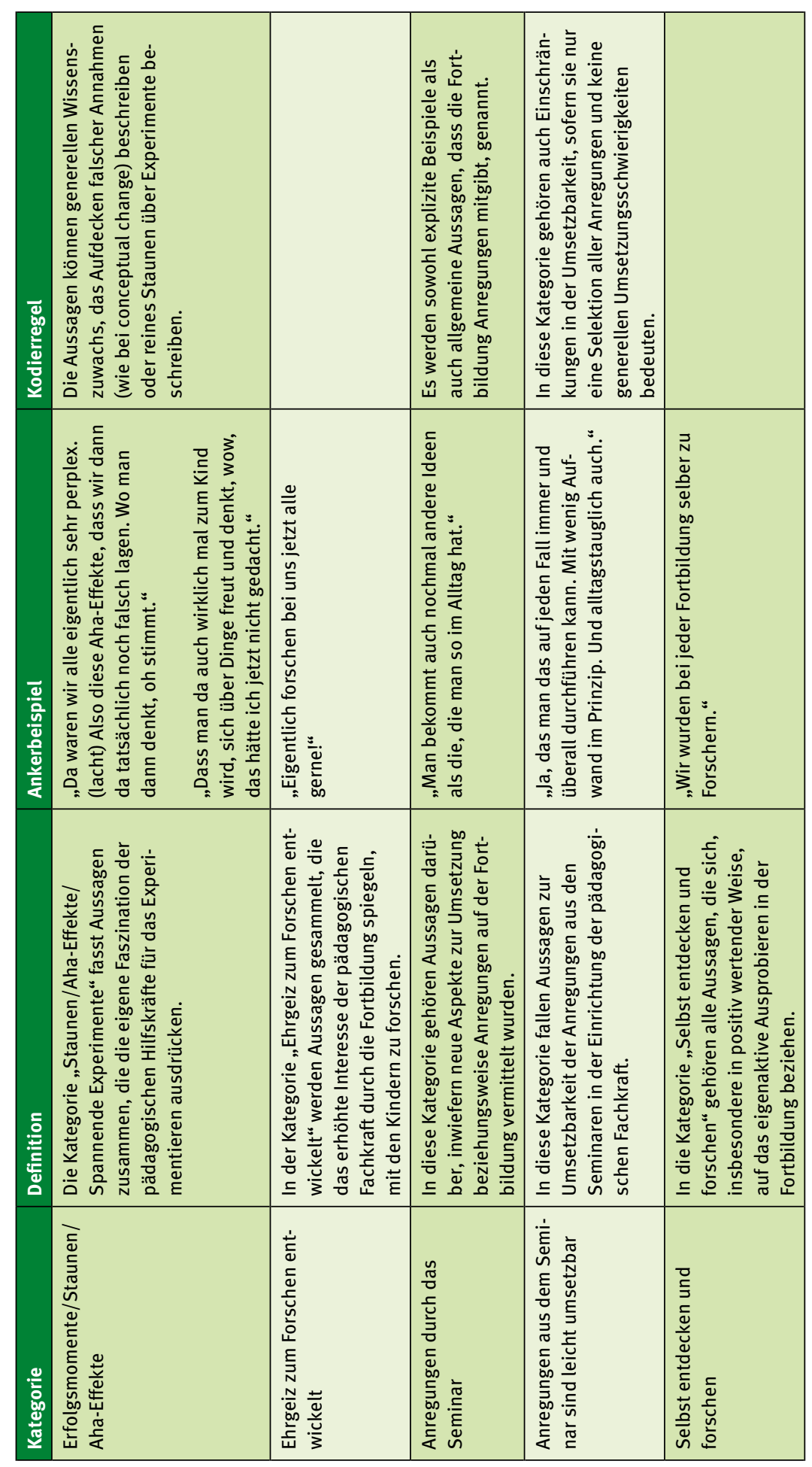



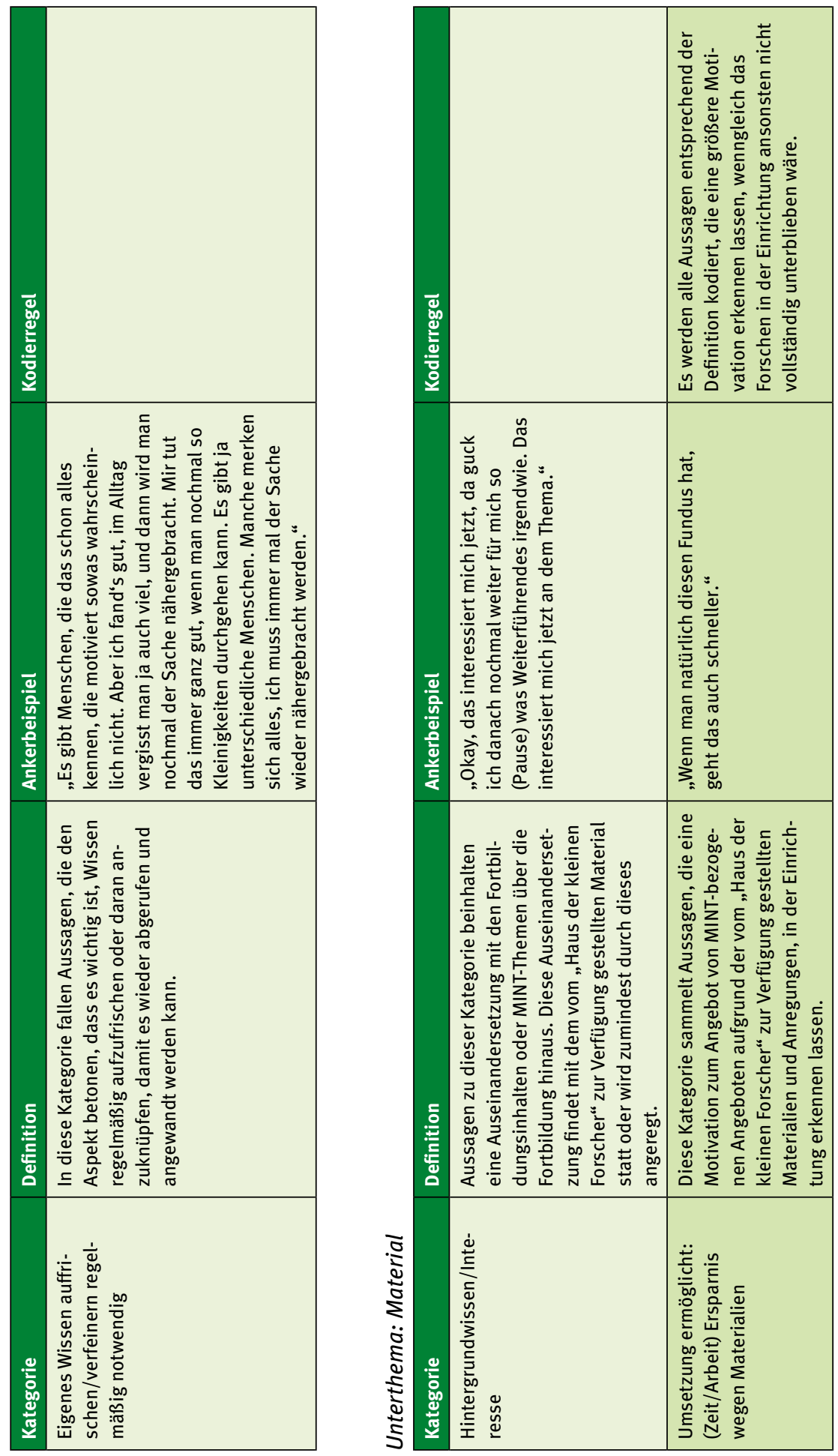

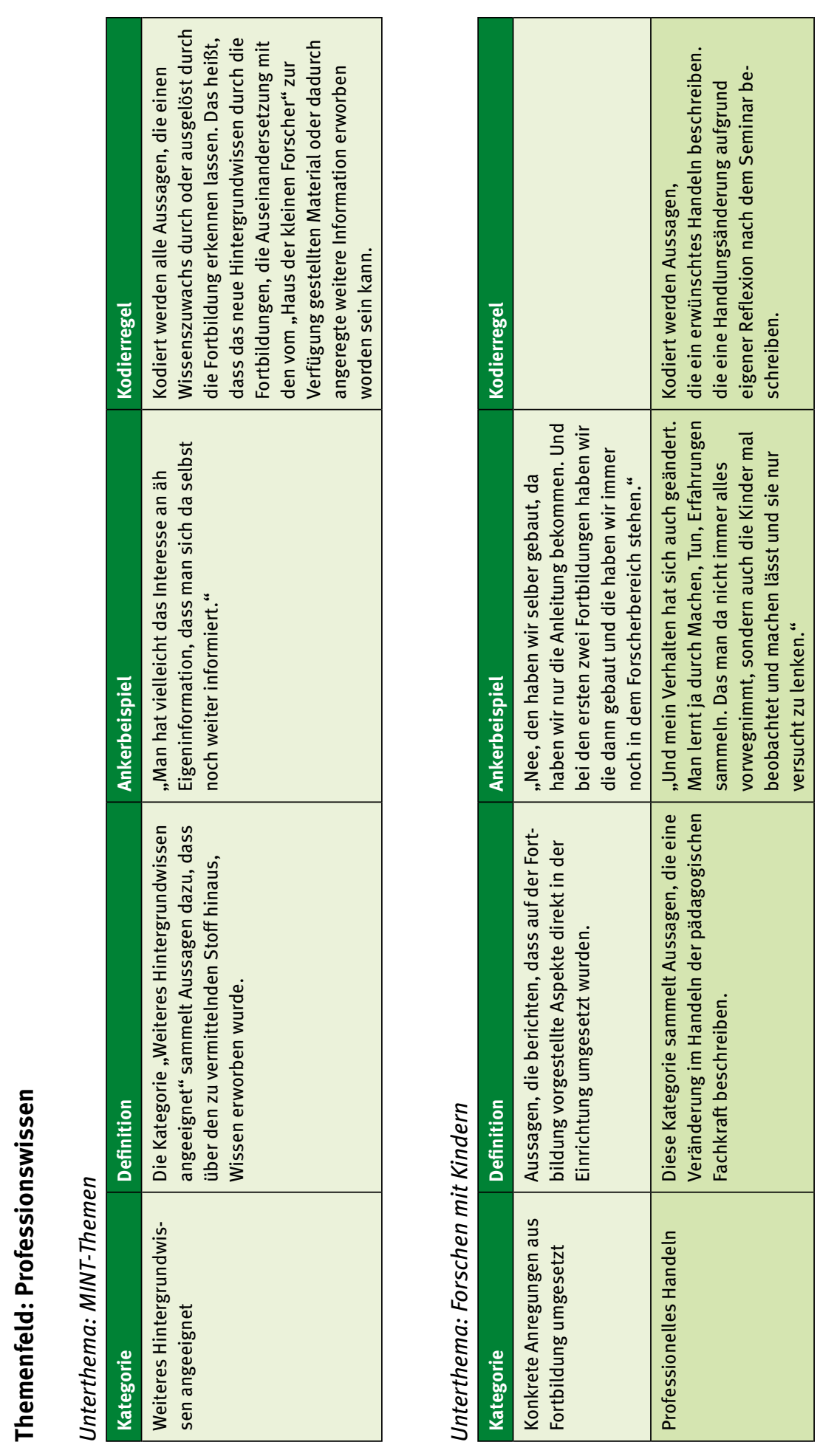


\begin{tabular}{|c|c|c|c|c|c|}
\hline & 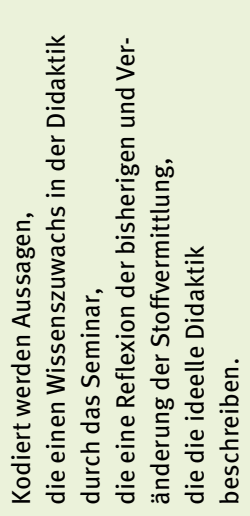 & 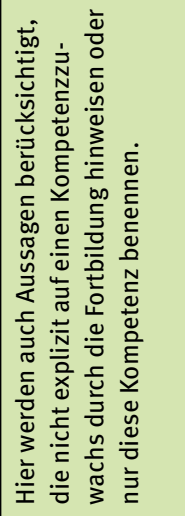 & 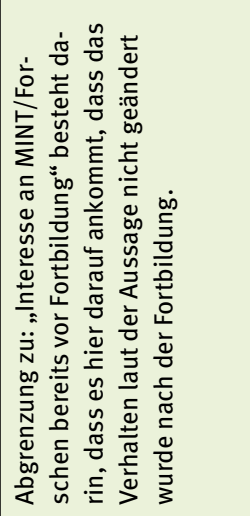 & & 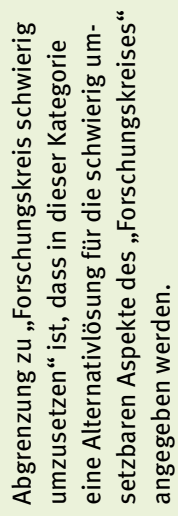 \\
\hline 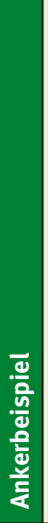 & 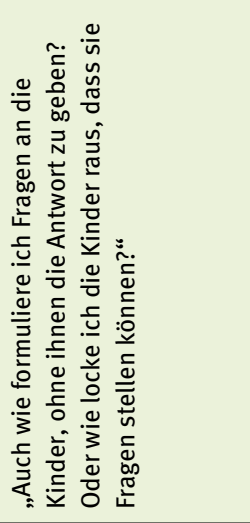 & 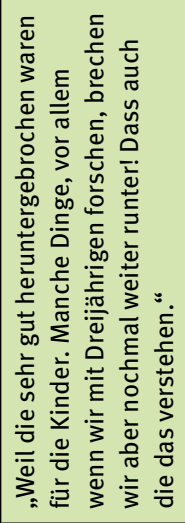 & 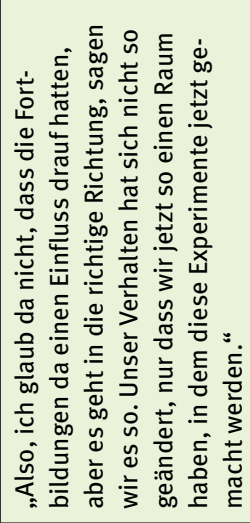 & 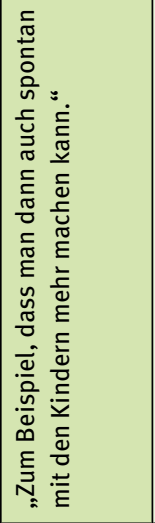 & 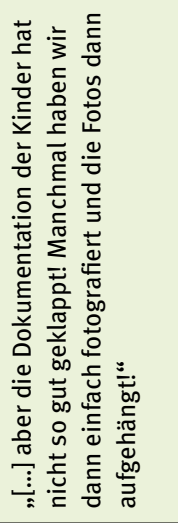 \\
\hline & 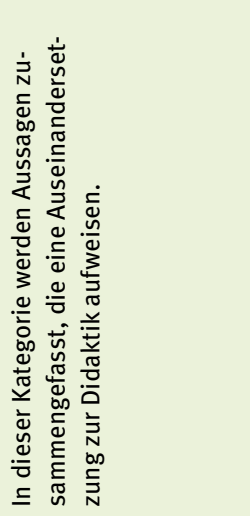 & 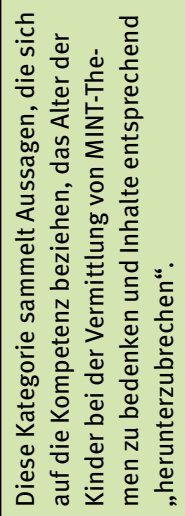 & 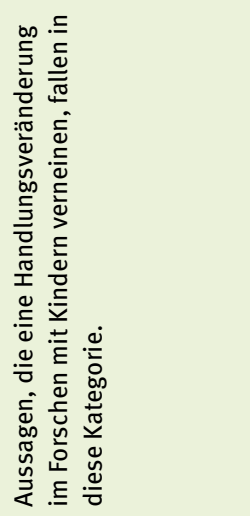 & 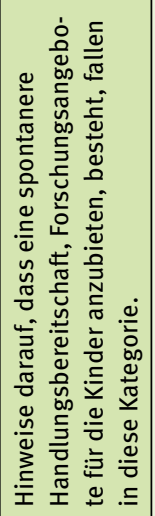 & 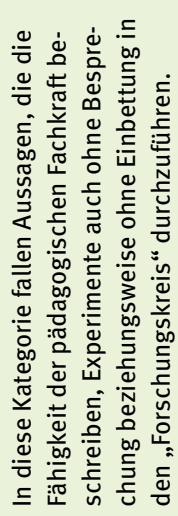 \\
\hline 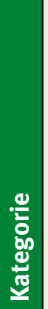 & 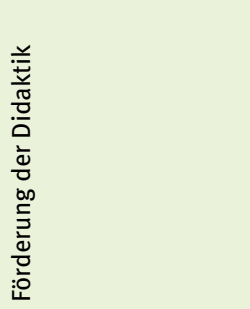 & 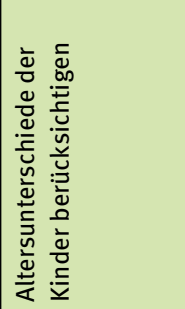 & 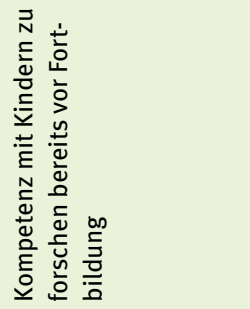 & 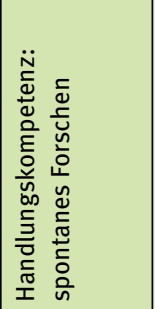 & 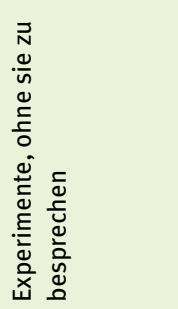 \\
\hline
\end{tabular}




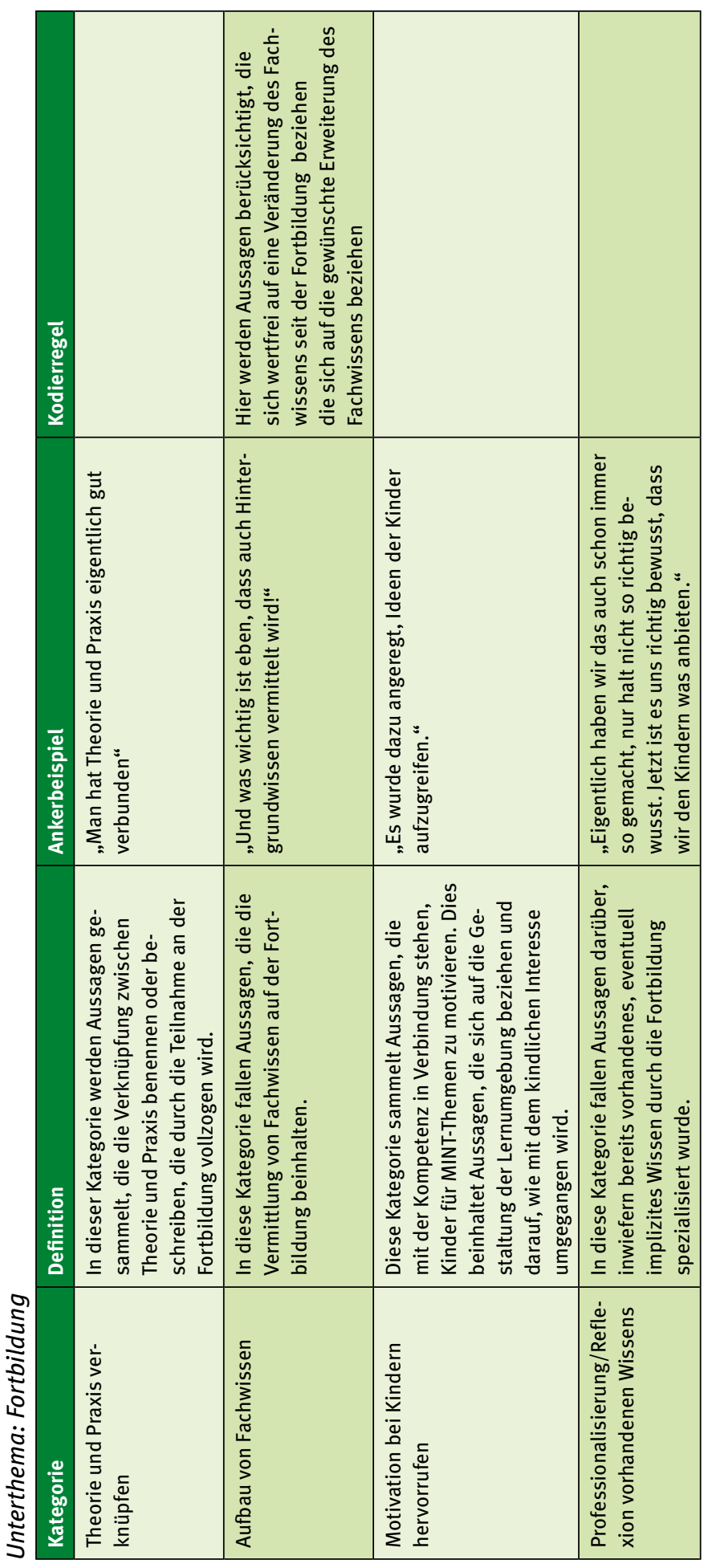




\begin{tabular}{|c|c|c|c|}
\hline 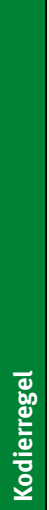 & 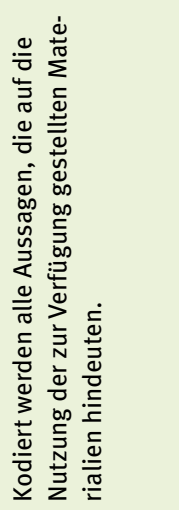 & 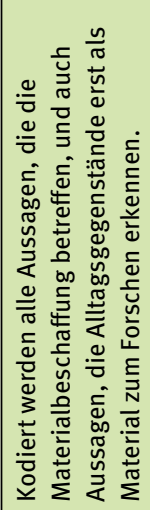 & 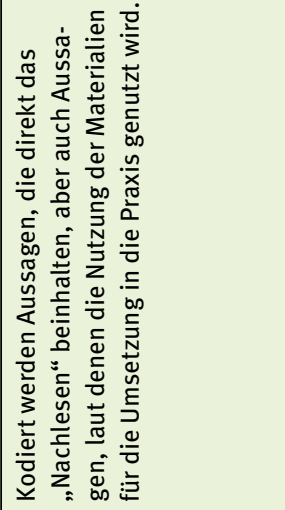 \\
\hline 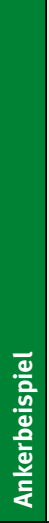 & 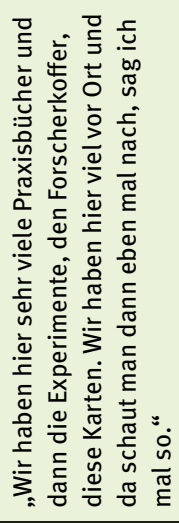 & 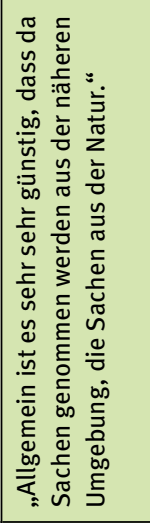 & 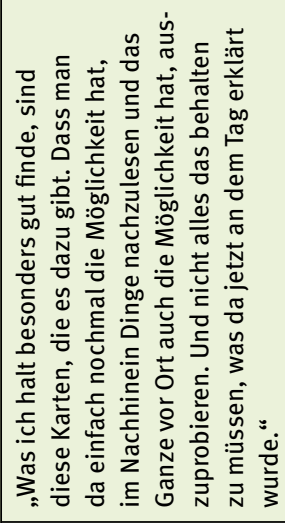 \\
\hline & 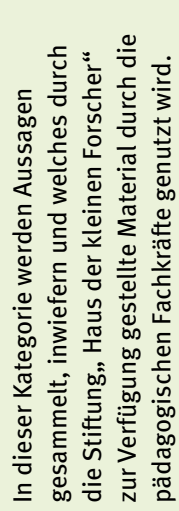 & 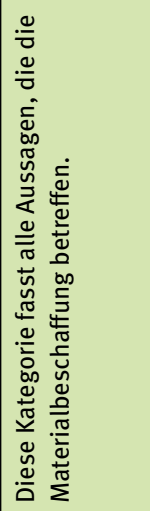 & 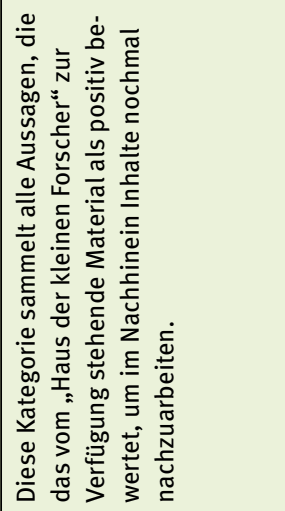 \\
\hline 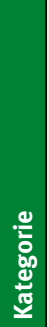 & 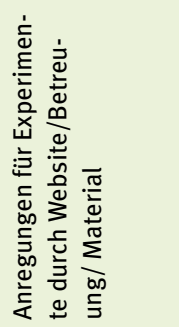 & 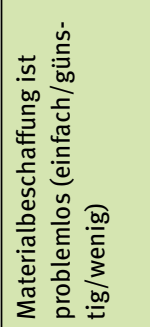 & 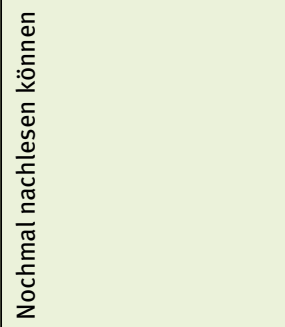 \\
\hline
\end{tabular}




\section{Bildquellenverzeichnis}

Seite 11: ( Heidi Scherm/Stiftung Haus der kleinen Forscher Seite 16, 38, 46, 69, 126, 164, 174, 192, 196, 208: @ Christoph Wehrer/Stiftung Haus der kleinen Forscher Seite 21: () Thomas Ernst/Stiftung Haus der kleinen Forscher Seite 34: (c) Tom Maelsa/Stiftung Haus der kleinen Forscher Seite 36, 168: () Birte Filmer/Stiftung Haus der kleinen Forscher Seite 59: (c) Frank Bentert/Stiftung Haus der kleinen Forscher Seite 120: @ Clara Teich/Stiftung Haus der kleinen Forscher 


\section{Über die Stiftung „Haus der kleinen Forscher“}

Die gemeinnützige Stiftung „Haus der kleinen Forscher“ engagiert sich für gute frühe Bildung in den Bereichen Mathematik, Informatik, Naturwissenschaften und Technik (MINT) - mit dem Ziel, Mädchen und Jungen stark für die Zukunft zu machen und zu nachhaltigem Handeln zu befähigen. Gemeinsam mit ihren Netzwerkpartnern vor Ort bietet die Stiftung bundesweit ein Bildungsprogramm an, das pädagogische Fach- und Lehrkräfte dabei unterstützt, Kinder im Kita- und Grundschulalter qualifiziert beim Entdecken, Forschen und Lernen zu begleiten. Das „Haus der kleinen Forscher“ verbessert Bildungschancen, fördert Interesse am MINT-Bereich und professionalisiert dafür pädagogisches Personal. Partner der Stiftung sind die Helmholtz-Gemeinschaft, die Siemens Stiftung, die Dietmar Hopp Stiftung und die Deutsche Telekom Stiftung. Gefördert wird sie vom Bundesministerium für Bildung und Forschung.

\section{Vision und Mission der Stiftung „Haus der kleinen Forscher"}

Vision der Stiftung „Haus der kleinen Forscher“:

Fragen - Forschen - Zukunft gestalten

Alle Kinder in Deutschland erleben Bildungsorte, in denen sie ihren eigenen Fragen nachgehen und forschend die Welt entdecken können. Solche „Häuser der kleinen Forscher“ machen Mädchen und Jungen stark für die Zukunft. Sie befähigen Kinder, selbstbestimmt zu denken und verantwortungsvoll zu handeln.

Technologisierung und Digitalisierung sowie Folgen des Klimawandels und der sozialen Ungleichheit beeinflussen zunehmend unseren Alltag. Wir tragen dazu bei, dass sich Menschen in unserer schnell verändernden Welt orientieren können und offen für Neues bleiben.

Die alltägliche Auseinandersetzung mit Natur und Technik fördert Neugier, Lern- und Denkfreude der Mädchen und Jungen. Wir sehen frühe Bildung als Schlüssel, um den Herausforderungen einer komplexen Welt erfolgreich begegnen zu können. 


\section{Mission der Stiftung „Haus der kleinen Forscher“:}

Die Stiftung „Haus der kleinen Forscher“...

befördert eine fragend-forschende Haltung bei Kindern,

- gibt Mädchen und Jungen schon in jungen Jahren die Chance, eigene Talente und Potenziale in den Bereichen Naturwissenschaften, Technik, Mathematik und Informatik zu entdecken

- und legt den Grundstein für einen reflektierten Umgang mit technologischen und gesellschaftlichen Veränderungen im Sinne einer nachhaltigen Entwicklung.

Gemeinsam mit ihren Bezugspersonen erleben die Kinder Spaß und Freude am Entdecken und Verstehen dieser Welt. Kinder gestalten Bildungsprozesse aktiv mit und erleben sich dadurch als kompetent und selbstwirksam in ihrem Alltag. Beim forschenden Lernen können Kinder Problemlösekompetenzen entwickeln, eigene Antworten finden und Selbstvertrauen spüren („Ich kann!“) - Erfahrungen und Fähigkeiten, die weit über die Kindheit hinaus für die Persönlichkeitsentwicklung und die spätere Berufsbiografie von Bedeutung sind.

In einem praxisnahen und qualitativ hochwertigen Professionalisierungsansatz unterstützt die Stiftung pädagogische Fach- und Lehrkräfte dabei, Kinder im Alter bis zehn Jahren beim Entdecken, Forschen und Lernen zu begleiten. Über vielfältige Fortbildungsangebote erleben Fach- und Lehrkräfte die Faszination eigenen Forschens für sich selbst. Sie erweitern ihre Kenntnisse und pädagogischen Kompetenzen und setzen sie in ihrer alläglichen Arbeit mit Kindern um.

Die Initiative unterstützt Bildungseinrichtungen darin, sich als „Ort des forschenden Lernens" nachhaltig weiterzuentwickeln und in diesem Sinn als „Haus der kleinen Forscher“ förderliche Lernumgebungen für Kinder zu schaffen. 


\section{Bisher erschienen in der Wissenschaftlichen Schriftenreihe der Stiftung „Haus der kleinen Forscher“}
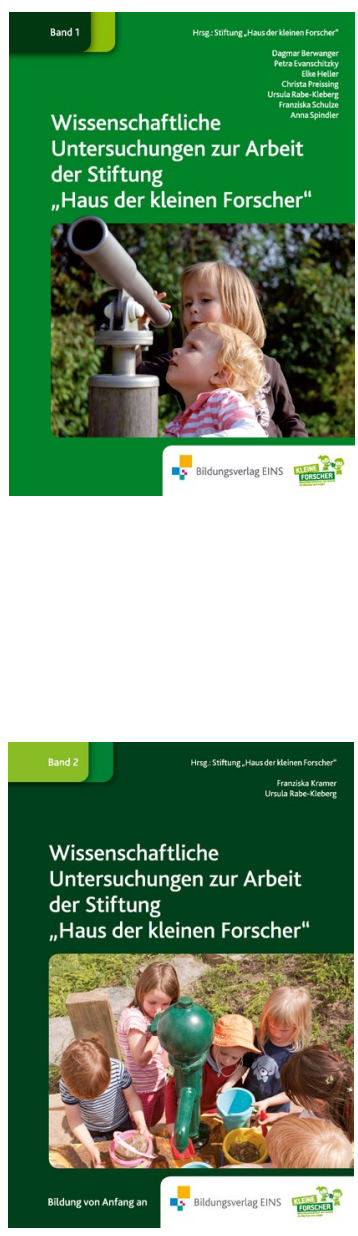

Band 1 (2011)

Dagmar Berwanger, Petra Evanschitzky, Elke Heller, Christa Preissing, Ursula Rabe-Kleberg, Franziska Schulze, Anna Spindler

Der erste Band der Schriftenreihe „Wissenschaftliche Untersuchungen zur Arbeit der Stiftung ,Haus der kleinen Forscher““ stellt vier wissenschaftliche Expertisen aus den Jahren 2009 und 2010 vor, die von renommierten Wissenschaftlerinnen und Wissenschaftlern aus dem Bereich der frühkindlichen Pädagogik verfasst wurden. Die vorliegenden wissenschaftlichen Beiträge reflektieren den pädagogischen Ansatz und das Multiplikatorenmodell der Stiftung vor dem Hintergrund der eigenen Zielvorstellungen, analysieren die Umsetzungspraxis in den Netzwerken und Kitas und zeigen weitere Entwicklungsmöglichkeiten auf.

\section{Band 2 (2011)}

Franziska Kramer, Ursula Rabe-Kleberg

Die Studie von Ursula Rabe-Kleberg und Franziska Kramer bildet eine logische Konsequenz und Ergänzung zur ersten Untersuchung der Autorinnen „Erzieherinnen und ihre Haltung zu Naturwissenschaft und Technik für Jungen und Mädchen“, die in Band 1 dieser Schriftenreihe veröffentlicht wurde (siehe oben). Die Autorinnen untersuchen die Gestaltung der Lernprozesse durch Erzieherinnen im Detail. Mit hoher Präzision und Sensibilität im Umgang mit den Möglichkeiten qualitativer Sozialforschung gelingt es den Autorinnen dabei, ko-konstruktive Augenblicke des gemeinsamen Lernens in Kitas einzufangen und intensiv im Hinblick auf wichtige Einflussvariablen zu reflektieren. 


\section{Band 3 (2012)}

Michael Fritz, Gabriele Grieshop, Katrin Hille, Maren Lau, Martin Winter

Im dritten Band werden zwei Studien vorgestellt, die sich mit der Rolle der Bedeutung der Trainerinnen und Trainer in der Initiative „Haus der kleinen Forscher“ aus jeweils unterschiedlichen Perspektiven beschäftigen: In der Studie von Maren Lau, Michael Fritz und Katrin Hille (ZNL) stehen das Rollen- und Selbstverständnis der Trainerinnen und Trainer sowie ihr subjektives Kompetenzempfinden im Mittelpunkt. In der Untersuchung von Gabriele Grieshop und Martin Winter (Institut für Didaktik der Mathematik und des Sachunterrichts (IFD), Universität Vechta) wird - im Rahmen einer vorwiegend formativen Implementierungsevaluation am Beispiel Mathematik - die Beteiligung der Trainerinnen und Trainer an der Konzept- und Materialentwicklung von Angeboten der Stiftung „Haus der kleinen Forscher" betrachtet.

\section{Band 4 (2012)}

\section{Salman Ansari, Susanna Jeschonek, Janna Pahnke, Sabina Pauen}

Band 4 enthält vier Expertisen, die basierend auf aktuellen entwicklungspsychologischen Erkenntnissen Empfehlungen für die Entwicklung weiterer naturwissenschaftlicher, technischer und mathematischer Themenschwerpunkte der Stiftung „Haus der kleinen Forscher“ aussprechen, auf mögliche Stolpersteine hinweisen und Vorschläge für die Praxis aufzeigen. Die Expertise von Janna Pahnke und Sabina Pauen gibt einen Überblick über die Entwicklung des mathematischen und naturwissenschaftlichen Denkens und Wissens in der frühen Kindheit und zieht Schlussfolgerungen für eine darauf aufbauende frühe Bildung in diesen Bereichen. Susanna Jeschoneks Expertisen behandeln die Entwicklung des kindlichen Verständnisses der Bereiche ,Magnetismus‘ und ,Akustik“ und geben Empfehlungen für die Aufbereitung von Bildungsangeboten zu diesen Themenschwerpunkten in der Praxis. In der Expertise von Salman Ansari stehen Prozesse des Lehrens und Lernens aus der Sicht der kognitiven Wissenschaften im Mittelpunkt. Ansari geht auf verschiedene Konzepte und Angebote der Stiftung „Haus der kleinen Forscher“ ein, spricht Empfehlungen für die Weiterentwicklung dieser Themen aus und verdeutlicht dies anhand von konkreten Beispielen für die praktische Umsetzung.
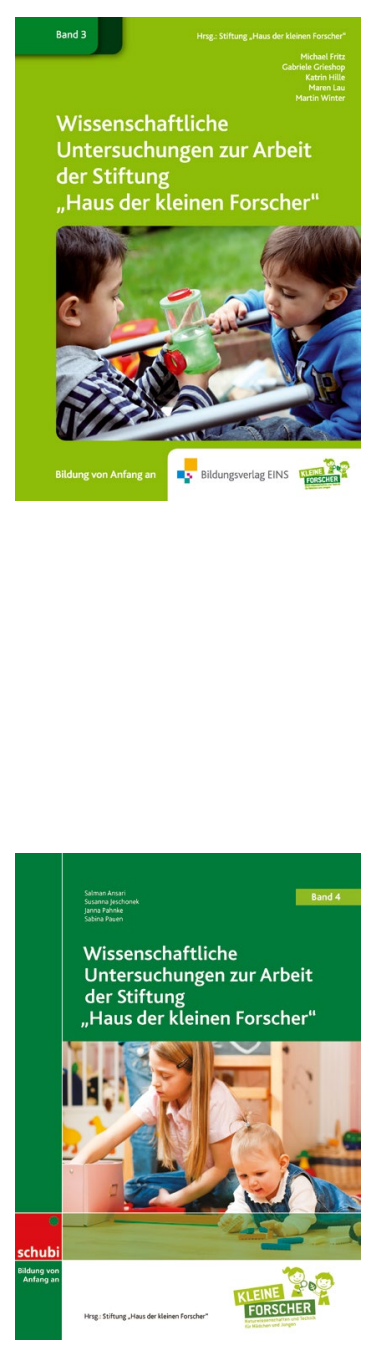


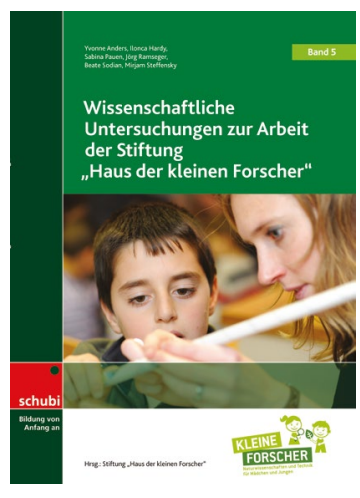

\section{Band 5 (2013)}

Yvonne Anders, llonca Hardy, Sabina Pauen, Jörg Ramseger, Beate Sodian, Mirjam Steffensky

Der fünfte Band stellt Ziele naturwissenschaftlicher Bildung für Kinder und pädagogische Fach-und Lehrkräfte sowie prozessbezogene Qualitätskriterien für den naturwissenschaftlichen Unterricht im Elementar- und Primarbereich in den Fokus. Yvonne Anders, Ilonca Hardy, Sabina Pauen, Beate Sodian und Mirjam Steffensky spezifizieren in ihren Expertisen pädagogisch-inhaltliche Zieldimensionen naturwissenschaftlicher Bildung im Kita- und Grundschulalter. Neben einer theoretischen Fundierung verschiedener Zielbereiche werden Instrumente für deren Messung aufgeführt. Jörg Ramseger formuliert in seiner Expertise zehn Qualitätskriterien für den naturwissenschaftlichen Unterricht. Diese prozessbezogenen Kriterien können pädagogische Fach- und Lehrkräfte bei der Unterrichtsplanung sowie bei der Selbstevaluation naturwissenschaftlicher Angebote im Elementar- und Primarbereich unterstützen.

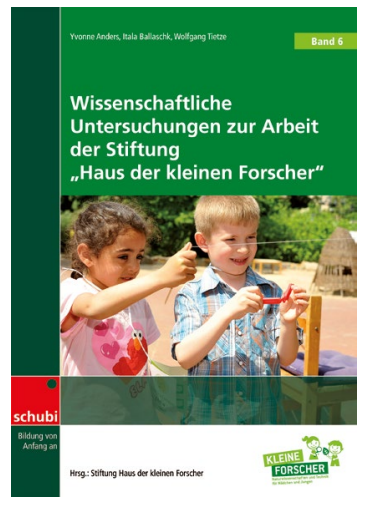

\section{Band 6 (2014)}

Yvonne Anders, Itala Ballaschk, Wolfgang Tietze

Im sechsten Band der Schriftenreihe mit einem Geleitwort von Wolfgang Tietze berichten Yvonne Anders und Itala Ballaschk die Ergebnisse ihrer Studie zur Untersuchung der Reliabilität und Validität des Zertifizierungsverfahrens der Stiftung „Haus der kleinen Forscher“, mit dem sich Bildungseinrichtungen nach bestimmten Qualitätskriterien als „Haus der kleinen Forscher“ zertifizieren lassen können. Insgesamt konnte in der Studie das Potenzial des Verfahrens für die Messung der naturwissenschaftsbezogenen Bildungsqualität in pädagogischen Einrichtungen belegt und Ansatzpunkte für weitere Optimierungen aufgezeigt werden. 


\section{Band 7 (2015)}

Gabriele Graube, Maja Jeretin-Kopf, Walter Kosack, Ingelore Mammes, Ortwin Renn, Christian Wiesmüller

Der siebte Band der Reihe mit Geleitwort von Ortwin Renn fokussiert Ziele und Konzepte technischer Bildung im Elementar- und Primarbereich. Walter Kosack, Maja Jeretin-Kopf und Christian Wiesmüller spezifizieren in ihrer Expertise pädagogisch-inhaltliche Zieldimensionen technischer Bildung im Kita- und Grundschulalter. Neben einer theoretischen Fundierung verschiedener Zielbereiche werden Instrumente für deren Messung aufgeführt. Die Autoren stellen in zwei Berichten die Ergebnisse empirischer Studien dar. Zum einen wurde der Einfluss verschiedener technikdidaktischer Materialsysteme auf die kindliche Motivation, problemlösendes Denken und technische Kreativität, und zum anderen der Einfluss verschiedener technikdidaktischer Methoden auf die kindliche Motivation sowie technikspezifische Denk- und Handlungsweisen untersucht. Gabriele Graube und Ingelore Mammes beschreiben in ihrem Beitrag ein didaktisches Konzept zur Unterstützung des professionellen Handelns pädagogischer Fach- und Lehrkräfte bei der Begleitung kindlicher Bildungsprozesse in ihrer Auseinandersetzung mit Natur und Technik.

\section{Band 8 (2017) \\ Christiane Benz, Meike Grüßing, Jens Holger Lorenz, Kristina Reiss, Christoph Selter, Bernd Wollring}

Der achte Band der Reihe mit einem Geleitwort von Kristina Reiss stellt die Ziele und Gelingensbedingungen mathematischer Bildung im Elementar- und Primarbereich in den Fokus. Christiane Benz, Meike Grüßing, Jens Holger Lorenz, Christoph Selter und Bernd Wollring spezifizieren in ihrer Expertise pädagogisch-inhaltliche Zieldimensionen mathematischer Bildung im Kita- und Grundschulalter. Neben einer theoretischen Fundierung verschiedener Zielbereiche werden Instrumente für deren Messung aufgeführt. Des Weiteren erörtern die Autorinnen und Autoren Gelingensbedingungen für eine effektive und wirkungsvolle frühe mathematische Bildung in der Praxis. Sie geben zudem Empfehlungen für die Weiterentwicklung der Stiftungsangebote und die wissenschaftliche Begleitung der Stiftungsarbeit im Bereich Mathematik. Das Schlusskapitel des Bandes beschreibt die Umsetzung dieser fachlichen Empfehlungen in den inhaltlichen Angeboten der Stiftung „Haus der kleinen Forscher“.
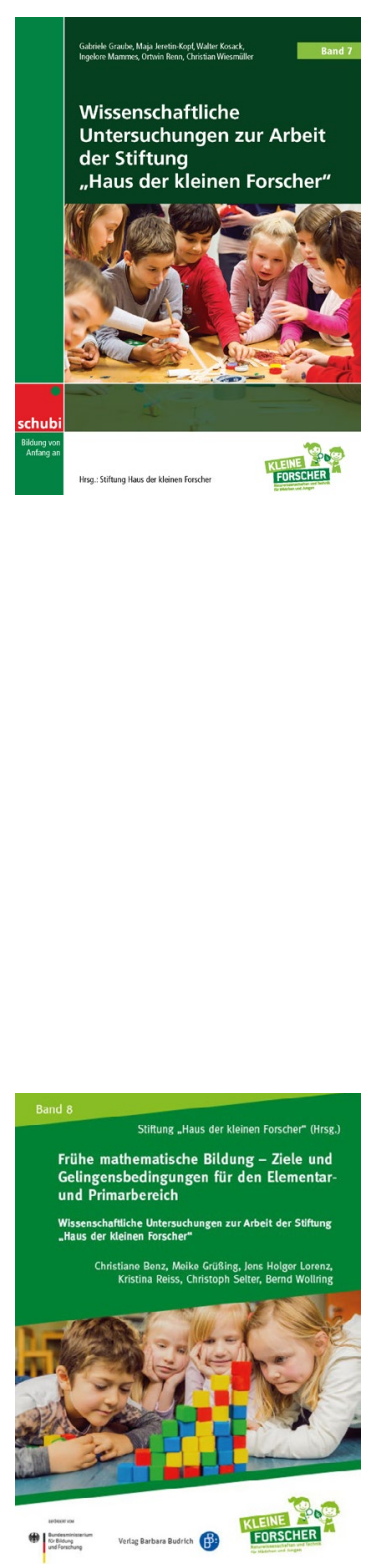


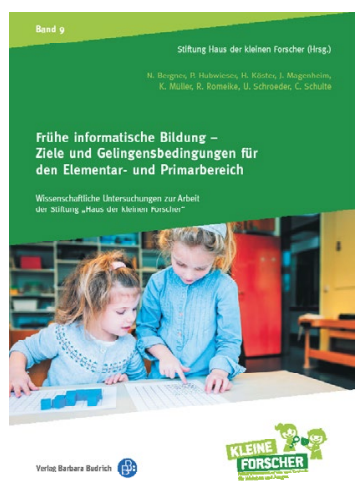

Band 9 (2018)

Nadine Bergner, Peter Hubwieser, Hilde Köster, Johannes Magenheim, Kathrin Müller, Ralf Romeike, Ulrik Schroeder, Carsten Schulte

Der neunte Band mit einem Geleitwort/Streitgespräch von Peter Hubwieser und Johannes Magenheim stellt die Ziele und Gelingensbedingungen informatischer Bildung im Elementar- und Primarbereich in den Fokus.

Nadine Bergner, Hilde Köster, Johannes Magenheim, Kathrin Müller, Ralf Romeike, Ulrik Schroeder und Carsten Schulte spezifizieren in ihrer Expertise pädagogisch-inhaltliche Zieldimensionen informatischer Bildung im Kita- und Grundschulalter. Neben einer theoretischen Fundierung verschiedener Zielbereiche erörtern die Autorinnen und Autoren Gelingensbedingungen für eine effektive und wirkungsvolle frühe informatische Bildung in der Praxis. Sie geben zudem Empfehlungen für die Entwicklung der Stiftungsangebote und die wissenschaftliche Begleitung der Stiftungsarbeit im Bereich Informatik. Nadine Bergner und Kathrin Müller beschreiben in ihrer Fachempfehlung eine Auswahl an Informatiksystemen für Kinder im Kita- und Grundschulalter und geben anhand fachlicher Kriterien Empfehlungen für besonders geeignete Informatiksysteme und deren Verwendung im Elementar- und Primarbereich.

Das Schlusskapitel des Bandes beschreibt die Umsetzung dieser fachlichen Empfehlungen in den inhaltlichen Angeboten der Stiftung „Haus der kleinen Forscher" mit und ohne Computer. 


\section{Band 10 (2018)}

Yvonne Anders, Julia Barenthien, Ilonca Hardy, Andreas Hartinger,

Miriam Leuchter, Elisa Oppermann, Sabina Pauen, Astrid Rank, Hans-Günther Roßbach, Mirjam Steffensky, Päivi Taskinen, Sabrina Tietze, Anja Wildemann, Tobias Ziegler

Der zehnte Band der Reihe mit einem Geleitwort von Hans-Günther Roßbach stellt die Ergebnisse der Studien zu Wirkungen naturwissenschaftlicher Bildungsangebote auf pädagogische Fachkräfte und Kinder im Vorschulalter vor. Mirjam Steffensky, Yvonne Anders, Julia Barenthien, Ilonca Hardy, Miriam Leuchter, Elisa Oppermann, Päivi Taskinen und Tobias Ziegler untersuchten die Wirkungen naturwissenschaftlicher Bildungsangebote auf die kognitiven und motivationalen Kompetenzen von pädagogischen Fachkräften und Kindern in Kitas. Insgesamt konnte aufgezeigt werden, dass die Teilnahme an naturwissenschaftlichen Fortbildungen in einem positiven Zusammenhang mit den naturwissenschaftlichen professionellen Kompetenzen pädagogischer Fachkräfte steht und dass Kinder aus Einrichtungen mit einem expliziten naturwissenschaftlichen Schwerpunkt höhere Lernfreude und höheres Selbstvertrauen in Bezug auf Naturwissenschaften zeigen als Kinder in Einrichtungen ohne naturwissenschaftlichen Schwerpunkt. Astrid Rank, Anja Wildemann, Sabina Pauen, Andreas Hartinger, Sabrina Tietze und Rahel Kästner untersuchten die Interaktionsqualität und mögliche sprachliche Bildungswirkungen im Kontext naturwissenschaftlicher Bildungsangebote bei Vorschulkindern. Die Studie legt nahe, dass naturwissenschaftliche Fortbildungen die naturwissenschaftlichen Kompetenzen wie auch den Spracherwerb der Kinder unterstützen und die Prozessqualität der Fachkräfte in naturwissenschaftsbezogenen Situationen bestimmen können. Das Schlusskapitel des Bandes beschreibt die Umsetzung der wissenschaftlichen Empfehlungen in den inhaltlichen Angeboten der Stiftung „Haus der kleinen Forscher“ und ihrer fachlichen Weiterentwicklung.

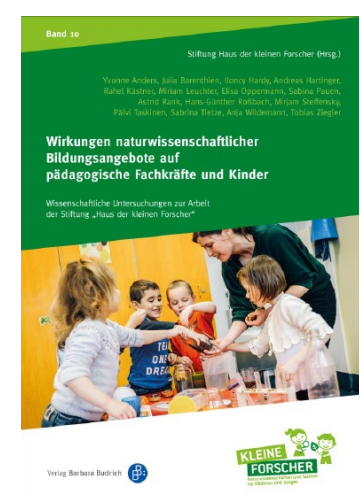




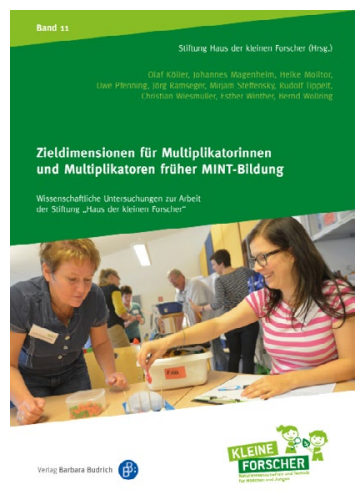

\section{Band 11 (2019)}

Olaf Köller, Johannes Magenheim, Heike Molitor, Uwe Pfenning, Jörg Ramseger, Mirjam Steffensky, Rudolf Tippelt, Christian Wiesmüller, Esther Winter, Bernd Wollring

Der elfte Band der Schriftenreihe mit einem Geleitwort von Rudolf Tippelt fokussiert die Zieldimensionen für Multiplikatorinnen und Multiplikatoren früher MINT-Bildung sowie einer frühen Bildung für nachhaltige Entwicklung. Olaf Köller und Esther Winther formulieren, basierend auf den Merkmalen erfolgreicher Lehrkräfte- und Erwachsenenbildung, ein Modell professioneller Kompetenz für Multiplikatorinnen und Multiplikatoren früher MINT-Bildung. Olaf Köller, Johannes Magenheim, Uwe Pfenning, Jörg Ramseger, Mirjam Steffensky, Christian Wiesmüller, Esther Winther und Bernd Wollring erörtern zentrale Zieldimensionen guter MINT-Erwachsenenbildnerinnen und bildner wie die motivationalen Orientierungen und Überzeugungen, die selbstregulativen Fähigkeiten, die fachspezifischen und fachdidaktischen sowie die pädagogisch-psychologischen Zieldimensionen des Professionswissens. Sie geben zudem Empfehlungen für die Priorisierung der Zieldimensionen und für die (Weiter-)Entwicklung der Stiftungsangebote. Heike Molitor kommentiert diese Zieldimensionen aus Sicht einer Bildung für nachhaltige Entwicklung und beschreibt, welchen Anforderungen Multiplikatorinnen und Multiplikatoren im Kontext einer (MINT-)Bildung für nachhaltige Entwicklung begegnen.

Das Fazit des Bandes beschreibt die Umsetzung der fachlichen Empfehlungen in die inhaltlichen Angebote der Stiftung „Haus der kleinen Forscher" und gibt einen Ausblick auf die weitere wissenschaftliche Begleitung der Stiftungsarbeit. 


\section{Band 12 (2019)}

Alexander Kauertz, Armin Lude, Heike Molitor, Andrea Saffran, Susanne Schubert, Mandy Singer-Brodowski, Daniela Ulber, Johannes Verch

Im Rahmen der Schriftenreihe „Wissenschaftliche Untersuchungen zur Arbeit der Stiftung „Haus der kleinen Forscher“ werden regelmäßig wissenschaftliche Beiträge von renommierten Expertinnen und Experten aus dem Bereich der frühen Bildung veröffentlicht. Diese Schriftenreihe dient einem fachlichen Dialog zwischen Stiftung, Wissenschaft und Praxis, mit dem Ziel, allen Kitas, Horten und Grundschulen in Deutschland fundierte Unterstützung für ihren Bildungsauftrag zu geben.

Der zwölfte Band der Schriftenreihe mit einem Geleitwort von Armin Lude fokussiert die Ziele und Gelingensbedingungen einer Bildung für nachhaltige Entwicklung für Kinder, pädagogische Fachkräfte und Leitungskräfte. Alexander Kauertz, Heike Molitor, Andrea Saffran, Susanne Schubert, Mandy Singer-Brodowski, Daniela Ulber und Johannes Verch erörtern in ihrer Expertise zentrale Zieldimensionen einer Bildung für nachhaltige Entwicklung auf der Ebene der Kinder sowie pädagogischer Fach- und Leitungskräfte. Neben einer theoretischen Fundierung identifizieren die Autorinnen und Autoren Gelingensbedingungen für eine effektive und wirkungsvolle frühe Bildung für nachhaltige Entwicklung in der Praxis. Sie geben zudem Empfehlungen für die Entwicklung der Stiftungsangebote und die wissenschaftliche Begleitung der Stiftungsarbeit zu BNE. Zwei weitere Kapitel beschreiben die Umsetzung der fachlichen Empfehlungen in die inhaltlichen Angebote der Stiftung „Haus der kleinen Forscher“ sowie die Evaluationsergebnisse zur Wirkung des Angebotsportfolios zu BNE. Ein Ausblick auf die zukünftige (Weiter-)Entwicklung der Stiftungsangebote zur Bildung für nachhaltige Entwicklung beschließt den Band.

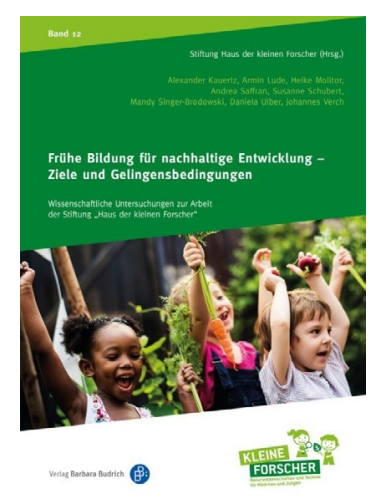




\section{Professionalisierung pädagogischer Fach- und Lehrkräfte in der frühen MINT-Bildung}

Im Rahmen der Schriftenreihe „Wissenschaftliche Untersuchungen zur Arbeit der Stiftung ,Haus der kleinen Forscher"“ werden regelmäßig wissenschaftliche Beiträge von renommierten Expertinnen und Experten aus dem Bereich der frühen Bildung veröffentlicht. Diese Schriftenreihe dient einem fachlichen Dialog zwischen Stiftung, Wissenschaft und Praxis, mit dem Ziel, allen Kitas, Horten und Grundschulen in Deutschland fundierte Unterstützung für ihren Bildungsauftrag zu geben.

Der dreizehnte Band der Schriftenreihe der Stiftung „Haus der kleinen Forscher“, mit einem Geleitwort von Mirjam Steffensky, fokussiert auf die professionelle Entwicklung von pädagogischen Fach- und Lehrkräften in der frühen MINT-Bildung. Nina Skorsetz, Lisa Öz, Julia Katharina Schmidt und Diemut Kucharz untersuchten in ihrer Studie Professionalisierungsprozesse und Lernbedarfe in der professionellen Entwicklung von Pädagoginnen und Pädagogen in der frühen MINT-Bildung. Dabei zeigte sich, dass die Anzahl besuchter MINT-Fortbildungen von zentraler Bedeutung für die Entwicklung der professionellen Kompetenz in der MINT-Bildung ist. Eine Längsschnittbefragung der Stiftung „Haus der kleinen Forscher" untersuchte die kurz- und mittelfristigen Wirkungen des Fortbildungsangebots auf die Kompetenzentwicklung von Pädagoginnen und Pädagogen und ihren Zusammenhang mit individuellen und organisationalen Rahmenbedingungen.

Das Schlusskapitel des Bandes beschreibt die Umsetzung der wissenschaftlichen Erkenntnisse und Empfehlungen in den inhaltlichen Angeboten der Stiftung "Haus der kleinen Forscher" und ihrer fachlichen Weiterentwicklung.

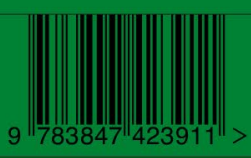

